Supporting Information

\title{
Gold Catalysts Can Generate Nitrone Intermediates from a Nitrosoarene/Alkene Mixture, Enabling Two Distinct Catalytic Reactions. A Nitroso-activated Cycloheptatriene/Benzylidene Rearrangement
}

Sayaji Arjun More ${ }^{a}$, Rahul Dadabhau Kardile ${ }^{a}$, Tung-Chun $\mathrm{Kuo}^{b}$, Mu-Jeng Cheng ${ }^{b *}$ and RaiShung $\operatorname{Liu}^{a *}$

${ }^{a}$ Department of Chemistry, National Tsing-Hua University, Hsinchu, Taiwan, ROC -rsliu@mx.nthu.edu.tw

${ }^{b}$ Department of Chemistry, National Cheng Kung University, Tainan, Taiwan, ROC e-mail: mjcheng@mail.ncku.edu.tw

\section{Content:}

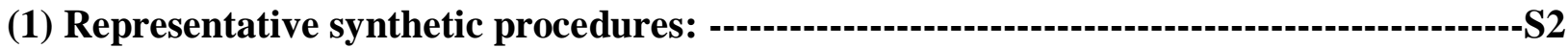

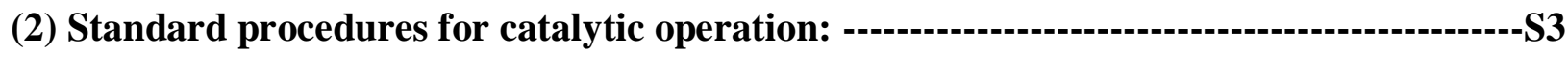

(3) Effects of nitrosoarenes on the overall oxidations (eq s1 and eq s2):--------------------S5

(4) Optimization table S1 for 5-ethoxy-3-methyl-2-phenylisoxazolidine (8a):----------------S6

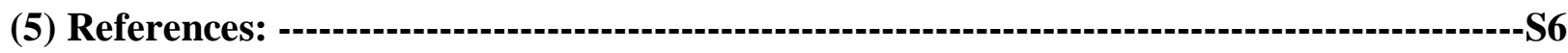

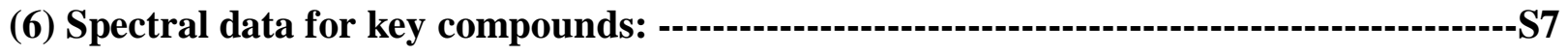

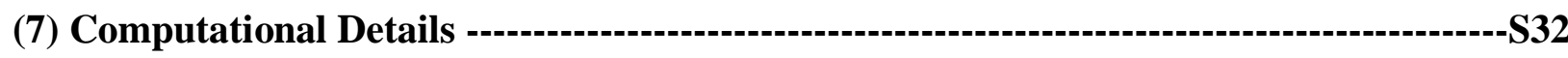

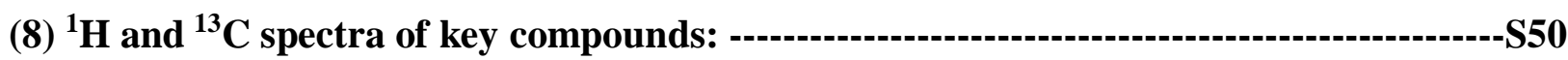




\section{Representative synthetic procedures:}

(a) General procedure:

Unless otherwise noted, all the reactions for the preparation of the substrates were performed in oven-dried glassware under nitrogen atmosphere with freshly distilled solvents. The catalytic reactions were performed under nitrogen atmosphere. DCM, DCE and toluene were distilled from $\mathrm{CaH}_{2}$ under nitrogen. THF was distilled from $\mathrm{Na}$ metal under nitrogen. All other commercial reagents were used without further purification, unless otherwise indicated. ${ }^{1} \mathrm{H} N \mathrm{NR}$ and ${ }^{13} \mathrm{C}$ NMR spectra were recorded on a Varian $700 \mathrm{MHz}$, Bruker 400, $600 \mathrm{MHz}$ spectrometers using chloroform- $d\left(\mathrm{CDCl}_{3}\right)$ as the internal standard. High-resolution mass spectral analysis (HRMS) data were measured on JMS-T100LP4G (JEOL) mass spectrometer or a TOF mass analyzer equipped with the ESI source and Magnetic Sector Mass Analyzer (MStation) equipped with the EI source. Nitroso compound (2b-2i) were prepared according to the reference. ${ }^{[1]}$ and all enol ethers (7) where parched from sigma Aldrich chemical company.

\section{(b) Preparation of 7-Aryl/alkyl-Cycloheptatrienes:}

7-Aryl-1,3,5-cycloheptatrienes (1a-1t) were prepared according to procedures reported in the literature. Characterization data can be found below for all the new substrates. ${ }^{[\mathrm{s} 2]}$

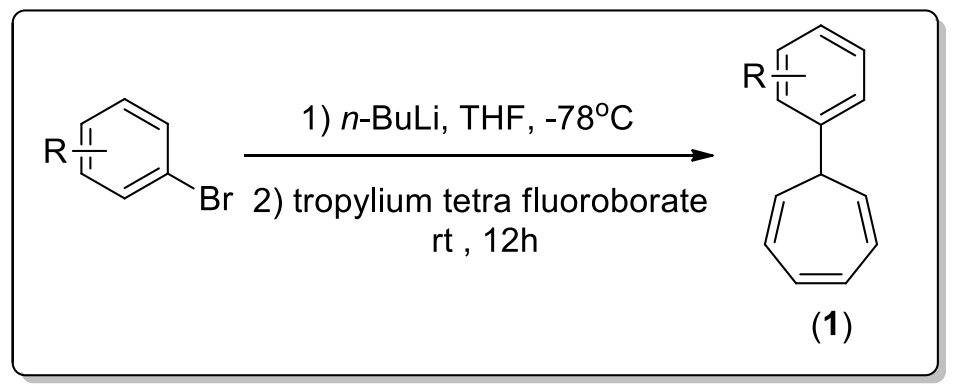

$n$-BuLi (2.5 $\mathrm{M}$ in hexanes, 1.2 equiv) was added dropwise to a solution of the corresponding aryl bromide (1.0 equiv) in dry $\mathrm{THF}(0.4 \mathrm{M})$ at $-78^{\circ} \mathrm{C}$ under argon. The mixture was stirred for $30 \mathrm{~min}$ at $-78{ }^{\circ} \mathrm{C}$, and then tropylium tetrafluoroborate (1.3 equiv) was added in one portion. The cooling bath was removed and the reaction was stirred at room temperature $\left(23^{\circ} \mathrm{C}\right)$ for $12 \mathrm{~h}$. The reaction was quenched with water. The aqueous phase was extracted with diethyl ether, the combined organic extracts were dried over $\mathrm{MgSO} 4$, and the solvent was evaporated. The crude reaction mixture was purified by column chromatography on silica gel with cyclohexane as eluent. 


\section{Standard procedures for catalytic operation:}

(a) Standard procedure for the synthesis of $(Z)$-N-benzylideneaniline oxide 3:

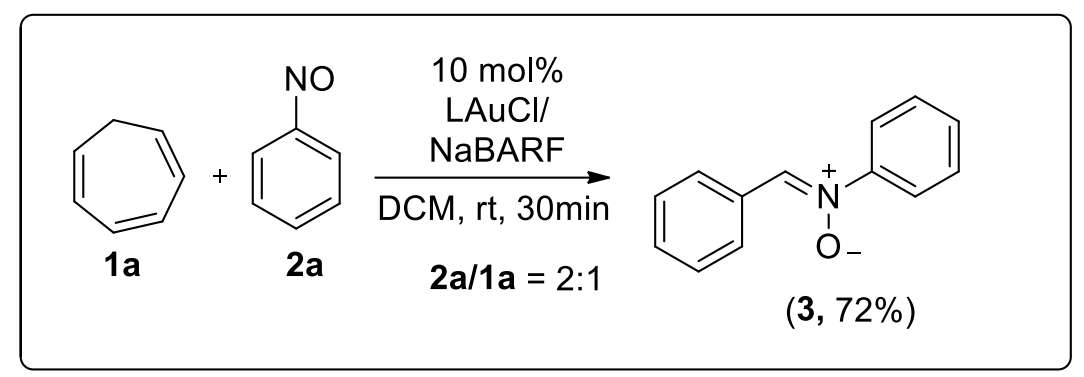

A suspension of $\mathrm{P}(t-\mathrm{Bu})_{2}\left(o\right.$-biphenyl)P-AuCl $(28.7 \mathrm{mg}, 0.054 \mathrm{mmol}), \mathrm{AgNTf}_{2}(21.05 \mathrm{mg}, 0.054$ mmol) and $4 \AA$ MS (40-50 mg) in dry DCM $(1 \mathrm{~mL})$ was fitted with $\mathrm{N}_{2}$ balloon and this mixture was stirred for $5 \mathrm{~min}$ at room temperature.To this solution was added a DCM (2 mL) solution of mixture of cycloheptatriens $1 \mathbf{a}(50 \mathrm{mg}, 0.54 \mathrm{mmol})$ and nitrosobenzene $\mathbf{2 a}(115.5 \mathrm{mg}, 1.08 \mathrm{mmol})$ and the reaction was further stirred at room temperature for $30 \mathrm{~min}$, the reaction mixture was filtered over a short celite bed, concentrated, and eluted through a silica column using ethyl acetate/hexane (30:70) as the eluent to give compound 3 in $72 \%$ yield $(77 \mathrm{mg})$. Spectral data of compound $\mathbf{3}$ is identical to those of authentic sample. ${ }^{[\mathrm{s} 3]}$

(b) Further transformation to 5-ethoxy-2,3-diphenylisoxazolidine (4):

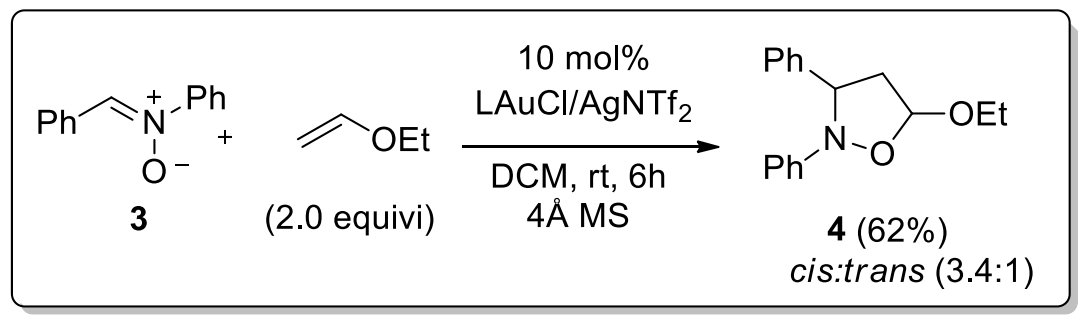

A suspension of $\mathrm{LAuCl} \mathrm{L=1,3-bis(diisopropylphenyl)} \mathrm{imidazol-2-ylidene} \mathrm{(16.7} \mathrm{mg,} 0.025 \mathrm{mmol}$ ) and $\operatorname{AgNTf}_{2}(9.8 \mathrm{mg}, 0.025 \mathrm{mmol})$ in dry DCM $(1.0 \mathrm{~mL})$ was fitted with a $\mathrm{N}_{2}$ balloon, and the mixture was stirred at $\mathrm{rt}$ for $5 \mathrm{~min}$. To this mixture was added dry DCM (2.0 mL) solution of (Z)N-benzylideneaniline oxide $3(50 \mathrm{mg}, 0.25 \mathrm{mmol})$ and vinyl ethyl ether $7 \mathbf{a}(36 \mathrm{mg}, 0.50 \mathrm{mmol})$ at $25^{\circ} \mathrm{C}$ and stirred for $6 \mathrm{~h}$. The solution was filtered over a short celite bed and evaporated under reduced pressure. The residue was purified on a silica gel column using ethyl acetate/hexane (07:93) 
as eluent to give compound 4 as yellow oil (44.0 $\mathrm{mg}, 0.16 \mathrm{mmol}, 62 \%)$. Spectral data of compound 4 is identical to those of authentic sample. ${ }^{[\mathrm{s} 4]}$

(c) Standard procedure for the synthesis of benzaldehyde 5a:

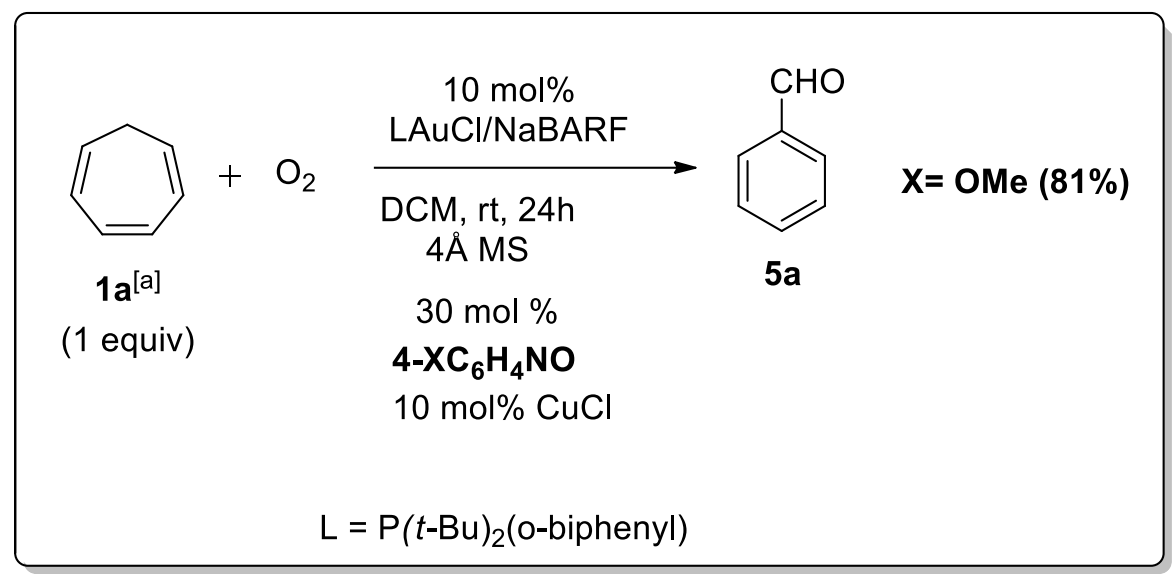

A catalytic tube was charged with $\mathrm{P}(t-\mathrm{Bu})_{2}(o$-biphenyl)P-AuCl $(28.7 \mathrm{mg}, 0.054 \mathrm{mmol}), \mathrm{NaBARF}$ (48.08 mg, $0.054 \mathrm{mmol}$ ), and $\mathrm{CuCl}$ (5.3 mg 0.054 mmole) and 4 Å MS (40-50 mg) in dry DCM $(0.5 \mathrm{~mL})$ was fitted with $\mathrm{O}_{2}$ balloon and this mixture was stirred for $5 \mathrm{~min}$ at room temperature. To this solution was added a DCM (2 mL) solution of mixture of cycloheptatriens $1 \mathbf{a}(50 \mathrm{mg}, 0.54$ $\mathrm{mmol}$ ) and 1-methoxy-2-nitrosobenzene $2 \mathrm{c}(148.1 \mathrm{mg}, 0.16 \mathrm{mmol})$ and the reaction was further stirred at room temperature for $24 \mathrm{~h}$, the reaction mixture was filtered over a short celite bed, concentrated, and eluted through a silica column using hexane as the eluent to give compound $\mathbf{5 a}$ in $81 \%$ yield $(46.5 \mathrm{mg})$. Spectral data of compound $\mathbf{5 a}$ is identical to those of authentic sample. ${ }^{\text {[s5] }}$

(d) Standard procedure for the synthesis of 5-ethoxy-3-methyl-2-phenylisoxazolidine 8a:

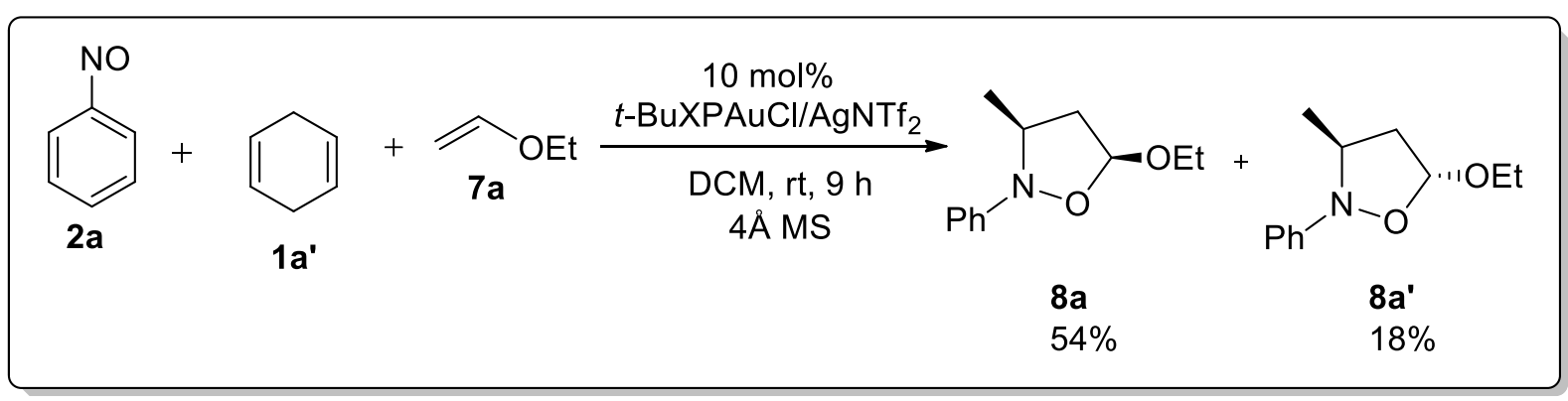

A suspension of (2-di-tert-butylphosphino-2',4',6'-triisopropylbiphenyl)P-AuCl (24.74 mg, 0.046 $\mathrm{mmol}), \operatorname{AgNTf}_{2}(18.11 \mathrm{mg}, 0.046 \mathrm{mmol})$ and $4 \AA$ MS (40-50 mg) in dry DCM (1.0 mL) was fitted with $\mathrm{N}_{2}$ balloon and this mixture was stirred for $5 \mathrm{~min}$ at room temperature. To this solution was 
added a DCM (2.0 mL) solution of mixture of nitrosobenzene 2a $(50 \mathrm{mg}, 0.46 \mathrm{mmol})$, cyclohexadine 1a' $(18.4 \mathrm{mg}, 0.23 \mathrm{mmol})$ and ethoxyethene $7 \mathbf{a}(66.2 \mathrm{mg}, 0.92 \mathrm{mmol})$ and the reaction was further stirred at room temperature for $9 \mathrm{~h}$, the reaction mixture was filtered over a short celite bed, concentrated, and eluted through a silica column using ethyl acetate/hexane (20:80) as the eluent to give compound $\mathbf{8 a}$ in $71 \%(68.5 \mathrm{mg})$.

\section{Effects of nitrosoarenes on the overall oxidations (eq s1 and eq s2):}
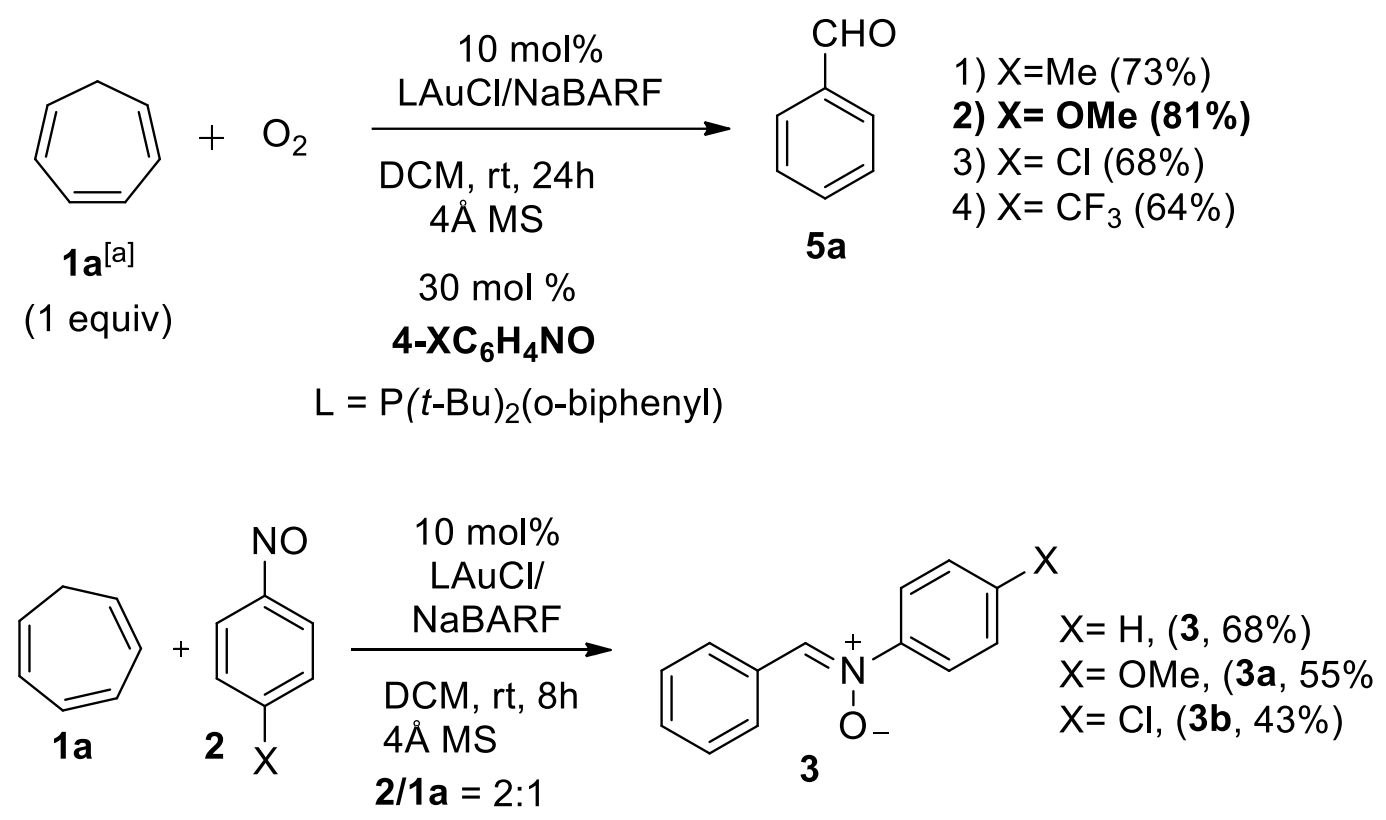

(eq s2)

Eq s1 presents the results of this oxidative rearrangement using various nitrosoarenes as cocatalysts. Among various para-substituents, we found that electron-rich substituents, $\mathrm{X}=\mathrm{OMe}$ and Me, gave yields (73-81 \%) of benzaldehyde 5a better than those (64-68 \%) of their electrondonating analogues, $\mathrm{X}=\mathrm{Cl}$ and $\mathrm{CF}_{3}$. According to a control experiment (eq s2), formation of nitrones 3 from a nitrosobenzene/ cycloheptatriene mixture is not the key step, because nitrosobenzene $(\mathrm{X}=\mathrm{H})$ gave the best yield of nitrone 3, compared to their 4-methoxy- and 4chlorophenylnitroso analogues. Herein, the $68 \%$ yield of nitrone 3 is less than that (76\%) of benzaldehyde 5a because of an excess amount of nitrosobenzene (1.0 equiv) in the former, further reducing the gold acidity. Hydration of nitrone $\mathbf{3}$ and reoxidation of $N$-hydroxyamine $\mathbf{6}$ seems to be more important for the entire sequence. 


\section{Table S1. Optimization table for 5-ethoxy-3-methyl-2-phenylisoxazolidine}

(8a):
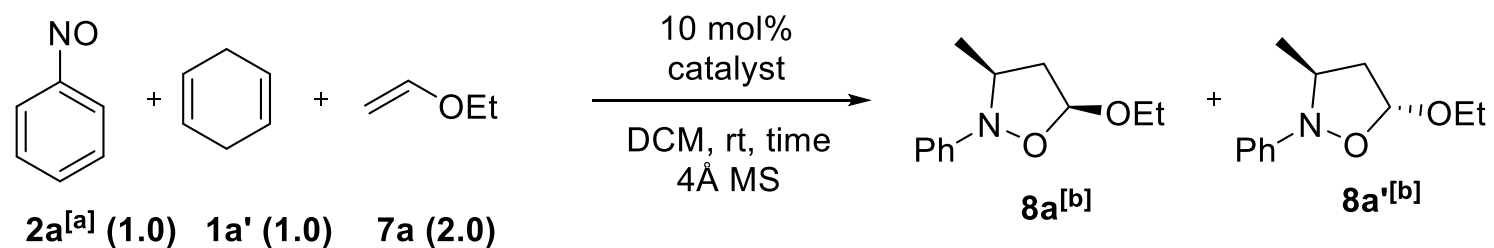

\begin{tabular}{|c|c|c|c|c|c|}
\hline Entry & Catalyst & Solvent & time (h) & $8 a \%$ & $8 a^{\prime} \%$ \\
\hline 1 & $\mathrm{LAuClAgNTf}_{2}$ & $\mathrm{DCM}$ & 12 & 40 & 16 \\
\hline 2 & $\mathrm{LAuClAgNTf}_{2}$ & toulene & 12 & 10 & 5 \\
\hline 3 & $t$-BuXPhosAuCl/AgNTf ${ }_{2}$ & $\mathrm{DCM}$ & 9 & 54 & 18 \\
\hline 4 & $t$-BuXPhosAuCl/AgOTf & DCM & 24 & 43 & 13 \\
\hline 5 & $t$-BuXPhosAuCl/AgSbF 6 & DCM & 24 & 43 & 10 \\
\hline 6 & $t$-BuXPhosAuCl/AgNTf 2 & toulene & 21 & 10 & 5 \\
\hline 7 & $t$-BuXPhosAuCl/AgNTf ${ }_{2}$ & DCE & 12 & 51 & 13 \\
\hline 8 & $t$-BuXPhosAuCl/AgNTf ${ }_{2}$ & THF & 12 & -- & -- \\
\hline
\end{tabular}

${ }^{a}[\mathbf{2 a}]=0.15 \mathrm{M}$. ${ }^{b}$ Product yields are obtained after purification from a silica column. $\mathrm{L}=\mathrm{P}(t-\mathrm{Bu})_{2}$ (o-biphenyl). $t$-BuXPhos =2-Di-tert-butylphosphino-2',4',6'-triisopropylbiphenyl.

\section{References:}

[s1] Compound (2b-2i): Zhao, D.; Jonhansson, M.; Backvall. J. Eur. J. Org. Chem. 2007, 26, 44314436.

[s2] (a) Solorio-Alvarado, C. R.; Wang, Y.; Echavarren, A. M. J. Am. Chem. Soc. 2011, 133, 11952-11955. (b) Wang, Y.; Muratore, M. E.; Rong, Z.; Echavarren, A. M. Angew. Chem., Int. Ed. 2014, 53, 14022-14026. (c) Yin, X.; Mato, M.; Echavarren, A. M. Angew. Chem., Int. Ed. 2017, 56, 14591-14595. (d) Nozos T.; Takashi K.; Yamamoto H. Bulletin of the Chemical society of Japan. 1969, 42, 3277-3284. (e) Bauld, N. L.; McDermed, J. D.; Hudson, C. E.; Rim, Y. S.; Zoeller, J.; Gordon, R. D.; Hyde, J. S. J. Am. Chem. Soc. 1969, 91, 6666-6676. (f) Jutz, C.; Voithenleitner, F. Chem. Ber. 1964, 97, 29-48.

[s3] Cisneros, L.; Serna, P.; Corma. Angew. Chem., Int. Ed. 2014, 53, 9306-9310.

[s4] Jiao, P.; Nakashima, D.; Yamamoto, H. Angew. Chem., Int. Ed. 2008, 47, 2411-2413.

[s5] Jiang L-Y.; Ming J-J.; Wang L-Y.; Jiang Y-Y.; Ren L-H.; Wanga Z-C.; Cheng W-C.Green Chem., 2020, 22, 1156-1163. 


\section{Spectral data for key compounds:}

\section{Spectral data of 7-(4-butylphenyl)cyclohepta-1,3,5-triene (1e):}

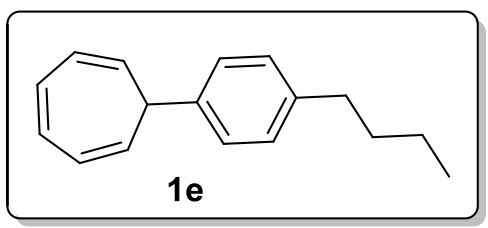

Colorless oil (1 g, 4.4 mmol, 79\%); ${ }^{1} \mathrm{H}$ NMR (400 MHz, CDCl3): $\delta 7.28$ (d, J=8.0 Hz, 2H), 7.19 $(\mathrm{d}, J=8.0 \mathrm{~Hz}, 2 \mathrm{H}), 6.75 \sim 6.73(\mathrm{~m}, 2 \mathrm{H}), 6.26 \sim 6.23(\mathrm{~m}, 2 \mathrm{H}), 5.42(\mathrm{dd}, J=9.2,5.2 \mathrm{~Hz}, 2 \mathrm{H}), 2.68$ $(t, J=5.3 \mathrm{~Hz}, 1 \mathrm{H}), 2.62(t, J=7.6 \mathrm{~Hz}, 2 \mathrm{H}), 1.63 \sim 1.57(\mathrm{~m}, 2 \mathrm{H}), 1.40 \sim 1.34(\mathrm{~m}, 2 \mathrm{H}), 0.94(t, J=$ $7.3 \mathrm{~Hz}, 3 \mathrm{H}) ;{ }^{13} \mathrm{C} \mathrm{NMR}(100 \mathrm{MHz}, \mathrm{CDCl}): \delta ; 141.2,141.1,130.9,128.7,127.4,126.5,124.3$, 44.9, 35.3, 33.7, 22.4, 14.0; HRMS (EI-MS) m/z: [M] ${ }^{+}$calcd for $\mathrm{C}_{17} \mathrm{H}_{20}$ : 224.1565; found: 224.1561 .

Spectral data of 2-(cyclohepta-2,4,6-trien-1-yl)thiophene (1p):

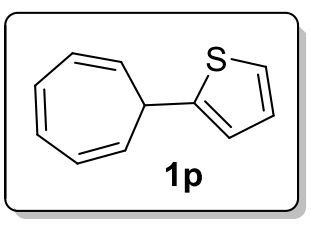

Colorless oil (0.8 g, $4.5 \mathrm{mmol}, 51 \%)$; ${ }^{1} \mathrm{H}$ NMR (400 MHz, CDCl3): $\delta 7.22 \sim 7.20(\mathrm{~m}, 1 \mathrm{H}), 7.03 \sim$ $7.0(\mathrm{~m}, 2 \mathrm{H}), 6.75 \sim 6.74(\mathrm{~m}, 2 \mathrm{H}), 6.28 \sim 6.25(\mathrm{~m}, 2 \mathrm{H}), 5.53 \sim 5.54(\mathrm{~m}, 2 \mathrm{H}), 3.0(t, J=5.6 \mathrm{~Hz}, 1 \mathrm{H})$; ${ }^{13} \mathrm{C}$ NMR (100 MHz, CDC13): $\delta$; 147.0, 131.2, 126.8, 125.6, 124.5, 123.6, 123.3, 40.5; HRMS (EI-MS) m/z: [M] $]^{+}$calcd for $\mathrm{C}_{11} \mathrm{H}_{10} \mathrm{~S}$ : 174.0503; found: 174.0500 .

Spectral data of 2-(cyclohepta-2,4,6-trien-1-yl)-4-methylthiophene (1q):

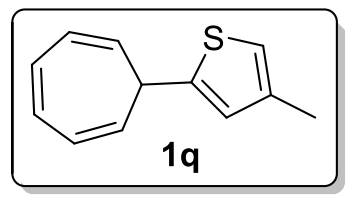

Colorless oil (0.45 g, $2.3 \mathrm{mmol}, 42 \%) ;{ }^{1} \mathrm{H}$ NMR (400 MHz, CDCl3): $\delta 6.82$ (s, 1H), $6.76 \sim 6.74$ (m, 1H), $6.73 \sim 6.71(\mathrm{~m}, 2 \mathrm{H}), 6.25 \sim 6.21(\mathrm{~m}, 2 \mathrm{H}), 5.49 \sim 5.46(\mathrm{~m}, 2 \mathrm{H}), 2.93(t, J=5.6 \mathrm{~Hz}, 1 \mathrm{H})$, 2.24 (s, 3H); ${ }^{13} \mathrm{C}$ NMR (100 MHz, CDCl3): $\delta ; 146.8,137.4,131.1,126.1,125.6,124.54,118.4$, 40.6, 15.8; HRMS (EI-MS) m/z: [M] ${ }^{+}$calcd for $\mathrm{C}_{12} \mathrm{H}_{12} \mathrm{~S}$ : 188.0660; found: 188.0656. 


\section{Spectral data of 2-(cyclohepta-2,4,6-trien-1-yl)furan (1r):}

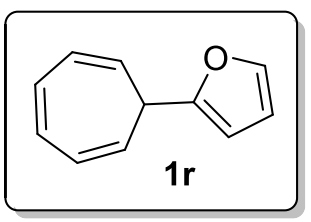

Pale yellow oil (0.44 g, $6.3 \mathrm{mmol}, 49 \%) ;{ }^{1} \mathrm{H}$ NMR (400 MHz, CDCl3): $\delta 7.39 \sim 7.38(\mathrm{~m}, 1 \mathrm{H})$, $6.71(t, J=3.3 \mathrm{~Hz}, 2 \mathrm{H}), 6.36 \sim 6.35(\mathrm{~m}, 1 \mathrm{H}), 6.27 \sim 6.21(\mathrm{~m}, 3 \mathrm{H}), 5.50 \sim 5.46(\mathrm{~m}, 2 \mathrm{H}), 2.88(t, J$ $=5.4 \mathrm{~Hz}, 1 \mathrm{H}) ;{ }^{13} \mathrm{C} \mathrm{NMR}(100 \mathrm{MHz}, \mathrm{CDCl} 3): \delta ; 156.2,141.5,131.1,124.9,123.0,110.2,104.8$, 39.1; HRMS (EI-MS) m/z: [M] calcd for $\mathrm{C}_{11} \mathrm{H}_{10} \mathrm{O}: 158.0732$; found: 158.0725 .

Spectral data of 5-ethoxy-2,3-diphenylisoxazolidine (4):

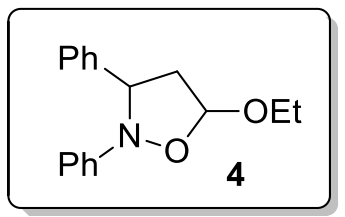

Compound 4 was purified on silica gel column using ethyl acetate/hexane: (7:93) as the eluent; Colorless oil (90.7 mg, $0.33 \mathrm{mmol}, 62 \%)$; Major isomer ${ }^{1} \mathrm{H}$ NMR (700 MHz, $\left.\mathrm{CDCl}_{3}\right): \delta 7.50$ (d, $J$ $=7.4 \mathrm{~Hz}, 2 \mathrm{H}), 7.36 \sim 7.32(\mathrm{~m}, 2 \mathrm{H}), 7.27 \sim 7.25(\mathrm{~m}, 1 \mathrm{H}), 7.17 \sim 7.14(\mathrm{~m}, 2 \mathrm{H}), 7.93 \sim 6.90(\mathrm{~m}, 3 \mathrm{H})$, $5.34(\mathrm{dd}, J=6,2 \mathrm{~Hz}, 1 \mathrm{H}), 4.30 \sim 4.28(\mathrm{~m}, 1 \mathrm{H}), 4.00 \sim 3.95(\mathrm{~m}, 1 \mathrm{H}), 3.62 \sim 3.58(\mathrm{~m}, 1 \mathrm{H}), 3.03 \sim$ $2.99(\mathrm{~m}, 1 \mathrm{H}), 2.38 \sim 2.35(\mathrm{~m}, 1 \mathrm{H}), 1.27(t, J=7 \mathrm{~Hz}, 3 \mathrm{H}) ;{ }^{1} \mathrm{H}$ NMR minor isomer: $\delta 7.47(\mathrm{~d}, J=$ $7.4 \mathrm{~Hz}, 2 \mathrm{H}), 7.36 \sim 7.32(\mathrm{~m}, 2 \mathrm{H}), 7.27 \sim 7.25(\mathrm{~m}, 1 \mathrm{H}), 7.17 \sim 7.14(\mathrm{~m}, 2 \mathrm{H}), 6.87 \sim 6.85(\mathrm{~m}, 1 \mathrm{H})$, $5.34(\mathrm{~d}, J=4.4 \mathrm{~Hz}, 1 \mathrm{H}), 4.80 \sim 4.77(\mathrm{~m}, 1 \mathrm{H}), 3.84 \sim 3.80(\mathrm{~m}, 1 \mathrm{H}), 3.54 \sim 3.52(\mathrm{~m}, 1 \mathrm{H}), 2.78 \sim$ $2.75(\mathrm{~m}, 1 \mathrm{H}), 2.46 \sim 2.42(\mathrm{~m}, 1 \mathrm{H}), 1.04(t, J=7 \mathrm{~Hz}, 3 \mathrm{H})$, two proton merged with major isomer peaks. Major isomer ${ }^{13} \mathrm{C}$ NMR (175 MHz, $\left.\mathrm{CDCl}_{3}\right): \delta 150.2,141.3,128.7,128.4,127.5,127.4$, $122.4,116.5,100.5,69.1,63.5,46.8,15.1 ;{ }^{13} \mathrm{C}$ NMR minor isomer $\delta 153.0,141.7,128.8,128.4$, 127.4, 126.4, 120.9, 113.9, 102.1, 67.4, 63.3, 47.1, 14.8; HRMS (EI-MS) m/z: [M] ${ }^{+}$calcd for $\mathrm{C}_{17} \mathrm{H}_{19} \mathrm{NO}_{2}: 269.1416$; found: 269.1418 .

\section{Spectral data of [1,1'-biphenyl]-4-carbaldehyde (5b-para):}




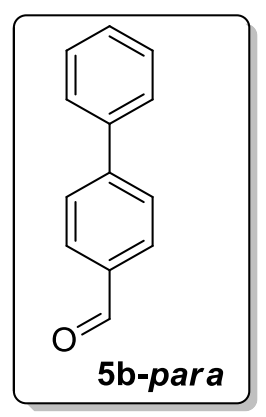

Compound (5b-para) was purified on silica gel column using hexane as the eluent; White solid (29.3 mg, $0.16 \mathrm{mmol}, 54 \%$ ); ${ }^{1} \mathrm{H}$ NMR (700 MHz, $\mathrm{CDCl}_{3}$ ): $\delta 10.04$ (s, $\left.1 \mathrm{H}\right), 7.94$ (d, $J=8.3 \mathrm{~Hz}$, $2 \mathrm{H}), 7.74(\mathrm{~d}, J=8.1 \mathrm{~Hz}, 2 \mathrm{H}), 7.63 \sim 7.61(\mathrm{~m}, 2 \mathrm{H}), 7.47 \sim 7.45(\mathrm{~m}, 2 \mathrm{H}), 7.41 \sim 7.39(\mathrm{~m}, 1 \mathrm{H}) ;{ }^{13} \mathrm{C}$ NMR (175 MHz, $\left.\mathrm{CDCl}_{3}\right): \delta 191.9,147.2,139.7,135.1,130.2,129.0,128.4,127.6,127.3 ;$ HRMS (ESI-TOF) m/z: $[\mathrm{M}+\mathrm{H}]^{+}$calcd for $\mathrm{C}_{13} \mathrm{H}_{10} \mathrm{O}: 183.0809$; found: 183.0817 .

\section{Spectral data of [1,1'-biphenyl]-2-carbaldehyde (5b-ortho):}

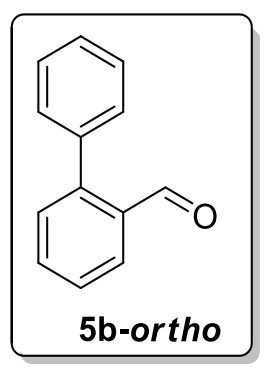

Compound (5b-ortho) was purified on silica gel column using hexane as the eluent; White solid (10.8 mg, $0.05 \mathrm{mmol}, 20 \%) ;{ }^{1} \mathrm{H} \mathrm{NMR}\left(700 \mathrm{MHz}, \mathrm{CDCl}_{3}\right): \delta 9.96(\mathrm{~s}, 1 \mathrm{H}), 8.01 \sim 8.00(\mathrm{~m}, 1 \mathrm{H})$, $7.63 \sim 7.61(\mathrm{~m}, 1 \mathrm{H}), 7.49 \sim 7.41(\mathrm{~m}, 5 \mathrm{H}), 7.37 \sim 7.36(\mathrm{~m}, 2 \mathrm{H}) ;{ }^{13} \mathrm{C} \mathrm{NMR}\left(175 \mathrm{MHz}, \mathrm{CDCl}_{3}\right): \delta$ 192.5, 145.9, 137.7, 133.7, 133.5, 130.7, 130.0, 128.4, 128.1, 127.7, 127.5.

Spectral data of 4'-methyl-[1,1'-biphenyl]-4-carbaldehyde (5c-para):

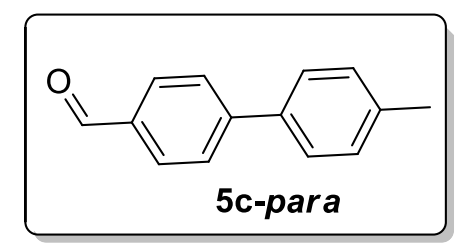

Compound (5c-para) was purified on silica gel column using hexane as the eluent; White solid (29.1 mg, $0.14 \mathrm{mmol}, 54 \%) ;{ }^{1} \mathrm{H}$ NMR (700 MHz, $\mathrm{CDCl}_{3}$ ): $\delta 10.02$ (s, $\left.1 \mathrm{H}\right), 7.91$ (d, $J=8.2 \mathrm{~Hz}$, $2 \mathrm{H}), 7.72(\mathrm{~d}, J=8.1 \mathrm{~Hz}, 2 \mathrm{H}), 7.52(\mathrm{~d}, J=8.0 \mathrm{~Hz}, 2 \mathrm{H}), 7.27(\mathrm{~d}, J=7.8 \mathrm{~Hz}, 2 \mathrm{H}), 2.39(\mathrm{~s}, 3 \mathrm{H}) ;{ }^{13} \mathrm{C}$ 
NMR (175 MHz, $\left.\mathrm{CDCl}_{3}\right): \delta 191.9,147.1,138.5,136.7,134.9,130.2,129.7,127.3,127.1,21.1$; HRMS (EI-MS) m/z: [M]+ calcd. For $\mathrm{C}_{14} \mathrm{H}_{12} \mathrm{O}: 196.0888$; found: 196.0896 .

Spectral data of 4'-methyl-[1,1'-biphenyl]-2-carbaldehyde (5c-ortho):

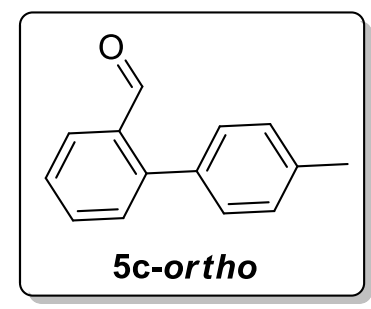

Compound (5c-ortho) was purified on silica gel column using hexane as the eluent; Light yellow liquid (14 mg, $0.07 \mathrm{mmol}, 26 \%) ;{ }^{1} \mathrm{H} \mathrm{NMR}\left(700 \mathrm{MHz}, \mathrm{CDCl}_{3}\right)$ : $\delta 9.97$ (s, $\left.1 \mathrm{H}\right), 8.0(\mathrm{~d}, J=7.7 \mathrm{~Hz}$, 1H), $7.61 \sim 7.59(\mathrm{~m}, 1 \mathrm{H}), 7.45(t, J=7.5 \mathrm{~Hz}, 1 \mathrm{H}), 7.42(\mathrm{~d}, J=7.6 \mathrm{~Hz}, 1 \mathrm{H}), 7.27 \sim 7.24(\mathrm{~m}, 4 \mathrm{H})$, 2.41 (s, 3H); ${ }^{13} \mathrm{C}$ NMR (175 MHz, $\left.\mathrm{CDCl}_{3}\right): \delta 192.6,145.9,138.0,134.7,133.7,133.5,130.7,130.0$, 129.1, 127.52, 127.11, 21.1.

Spectral data of 4'-(tert-butyl)-[1,1'-biphenyl]-4-carbaldehyde (5d-para):

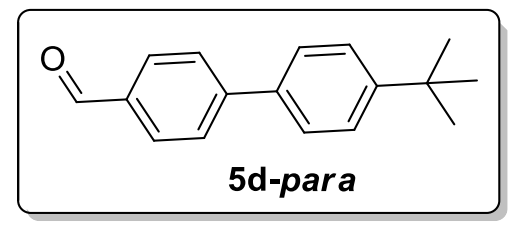

Compound (5d-para) was purified on silica gel column using hexane as the eluent; White solid (25.5 mg, $0.10 \mathrm{mmol}, 47 \%$ ); ${ }^{1} \mathrm{H}$ NMR (600 MHz, $\mathrm{CDCl}_{3}$ ): $\delta 10.03$ (s, $\left.1 \mathrm{H}\right), 7.92(\mathrm{~d}, J=8.1 \mathrm{~Hz}$, $2 \mathrm{H}), 7.73(\mathrm{~d}, J=8.1 \mathrm{~Hz}, 2 \mathrm{H}), 7.57(\mathrm{~d}, J=8.4 \mathrm{~Hz}, 2 \mathrm{H}), 7.49(\mathrm{~d}, J=8.4 \mathrm{~Hz}, 2 \mathrm{H}), 1.35(\mathrm{~s}, 9 \mathrm{H}) ;{ }^{13} \mathrm{C}$ NMR (175 MHz, $\left.\mathrm{CDCl}_{3}\right): \delta 191.9,151.7,147.0,136.7,134.9,130.2,127.4,127.0,125.9,34.6$, 31.2; HRMS (EI-MS) m/z: [M]+ calcd. For $\mathrm{C}_{17} \mathrm{H}_{18} \mathrm{O}: 238.1358$; found: 238.1369 .

Spectral data of 4'-(tert-butyl)-[1,1'-biphenyl]-2-carbaldehyde (5d-ortho):

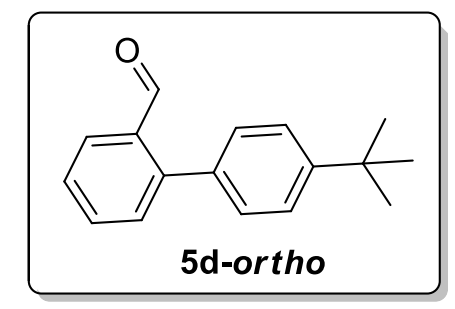

Compound (5d-ortho) was purified on silica gel column using hexane as the eluent; colorless oil (12.2 mg, $0.05 \mathrm{mmol}, 23 \%) ;{ }^{1} \mathrm{H} \mathrm{NMR}\left(400 \mathrm{MHz}, \mathrm{CDCl}_{3}\right): \delta 9.98(\mathrm{~s}, 1 \mathrm{H}), 8.01 \sim 7.99(\mathrm{~m}, 1 \mathrm{H})$, 
$7.63 \sim 7.59(\mathrm{~m}, 1 \mathrm{H}), 7.48 \sim 7.43(\mathrm{~m}, 4 \mathrm{H}), 7.31 \sim 7.29(\mathrm{~m}, 2 \mathrm{H}), 1.36(\mathrm{~s}, 9 \mathrm{H}) ;{ }^{13} \mathrm{C}$ NMR $(175 \mathrm{MHz}$, $\left.\mathrm{CDCl}_{3}\right): \delta 192.7,151.2,145.9,134.7,133.7,133.5,130.7,129.8,127.5,127.4,125.3,34.6,31.3$.

\section{Spectral data of 4'-butyl-[1,1'-biphenyl]-4-carbaldehyde (5e-para):}

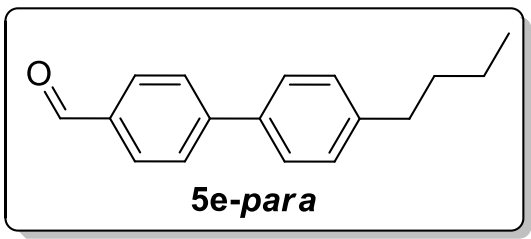

Compound (5e-para) was purified on silica gel column using hexane as the eluent; Yellow liquid (26 mg, $0.10 \mathrm{mmol}, 48 \%)$; ${ }^{1} \mathrm{H} \mathrm{NMR}$ (700 MHz, $\left.\mathrm{CDCl}_{3}\right): \delta 10.02(\mathrm{~s}, 1 \mathrm{H}), 7.91(\mathrm{~d}, J=8.1 \mathrm{~Hz}, 2 \mathrm{H})$, $7.72(\mathrm{~d}, J=8.1 \mathrm{~Hz}, 2 \mathrm{H}), 7.54(\mathrm{~d}, J=8.0 \mathrm{~Hz}, 2 \mathrm{H}), 7.27(\mathrm{~d}, J=7.9 \mathrm{~Hz}, 2 \mathrm{H}), 2.66(t, J=7.7 \mathrm{~Hz}$, 2H), $1.64 \sim 1.59(\mathrm{~m}, 2 \mathrm{H}), 1.39 \sim 1.36(\mathrm{~m}, 2 \mathrm{H}), 0.93(t, J=7.3 \mathrm{~Hz}, 3 \mathrm{H}) ;{ }^{13} \mathrm{C}$ NMR $(175 \mathrm{MHz}$, $\left.\mathrm{CDCl}_{3}\right): \delta 191.9,147.1,143.5,136.9,134.9,130.2,129.0,127.4,127.1,35.3,33.5,22.3,13.9$; HRMS (EI-MS) m/z: [M]+ calcd. For $\mathrm{C}_{17} \mathrm{H}_{18} \mathrm{O}: 238.1358$; found: 238.1356 .

Spectral data of 4'-butyl-[1,1'-biphenyl]-2-carbaldehyde (5e-ortho):

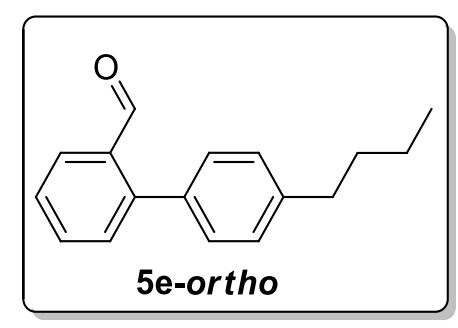

Compound (5e-ortho) was purified on silica gel column using hexane as the eluent; Yellow liquid (12.8 mg, $0.05 \mathrm{mmol}, 24 \%) ;{ }^{1} \mathrm{H} \mathrm{NMR}\left(700 \mathrm{MHz}, \mathrm{CDCl}_{3}\right): \delta 9.97(\mathrm{~s}, 1 \mathrm{H}), 7.98(t, J=7.0 \mathrm{~Hz}, 1 \mathrm{H})$, $7.61 \sim 7.58(\mathrm{~m}, 1 \mathrm{H}), 7.46 \sim 7.41(\mathrm{~m}, 2 \mathrm{H}), 7.26 \sim 7.23(\mathrm{~m}, 4 \mathrm{H}), 2.66(t, J=7.7 \mathrm{~Hz}, 2 \mathrm{H}), 1.65 \sim$ $1.57(\mathrm{~m}, 2 \mathrm{H}), 1.39 \sim 1.36(\mathrm{~m}, 2 \mathrm{H}), 0.93(t, J=7.3 \mathrm{~Hz}, 3 \mathrm{H}) ;{ }^{13} \mathrm{C} \mathrm{NMR}\left(175 \mathrm{MHz}, \mathrm{CDCl}_{3}\right): \delta 192.6$, 146.0, 143.0, 134.9, 133.7, 133.5, 130.7, 130.0, 128.4, 127.5, 127.4, 35.3, 33.5, 22.3, 13.9.

Spectral data of 4'-methoxy-[1,1'-biphenyl]-4-carbaldehyde (5f-para):

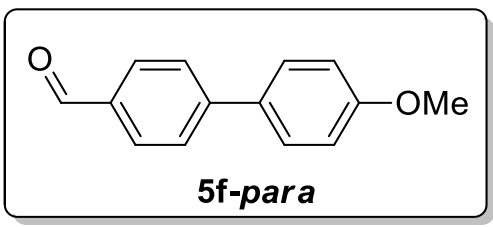

Compound (5f-para) was purified on silica gel column using hexane as the eluent; White solid (30 mg, $0.14 \mathrm{mmol}, 56 \%$ ); ${ }^{1} \mathrm{H}$ NMR (700 MHz, $\mathrm{CDCl}_{3}$ ): $\delta 10.01$ (s, $\left.1 \mathrm{H}\right), 7.90$ (d, J = 8.1 Hz, 2H), 7.69 
$(\mathrm{d}, J=8.1 \mathrm{~Hz}, 2 \mathrm{H}), 7.57(\mathrm{~d}, J=8.6 \mathrm{~Hz}, 2 \mathrm{H}), 6.99(\mathrm{~d}, J=8.6 \mathrm{~Hz}, 2 \mathrm{H}), 3.84(\mathrm{~s}, 3 \mathrm{H}) ;{ }^{13} \mathrm{C}$ NMR $(175$ $\mathrm{MHz}, \mathrm{CDCl}_{3}$ ): $\delta 191.8,160.0,146.7,134.6,132.0,130.2,128.4,127.0,114.4,55.3$; HRMS (EIMS) m/z: [M]+ calcd. For $\mathrm{C}_{14} \mathrm{H}_{12} \mathrm{O}_{2}: 212.0837$; found: 212.0836.

Spectral data of 4'-methoxy-[1,1'-biphenyl]-2-carbaldehyde (5f-ortho):

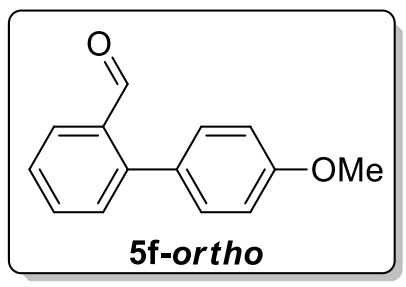

Compound (5f-ortho) was purified on silica gel column using hexane as the eluent; White solid (15 mg, $0.07 \mathrm{mmol}, 28 \%) ;{ }^{1} \mathrm{H} \mathrm{NMR}\left(700 \mathrm{MHz}, \mathrm{CDCl}_{3}\right): \delta 9.97$ (s, $\left.1 \mathrm{H}\right), 7.98(\mathrm{~d}, J=7.7 \mathrm{~Hz}, 1 \mathrm{H})$, $7.61 \sim 7.58(\mathrm{~m}, 1 \mathrm{H}), 7.45 \sim 7.40(\mathrm{~m}, 2 \mathrm{H}), 7.29(\mathrm{~d}, J=8.6 \mathrm{~Hz}, 2 \mathrm{H}), 6.98(\mathrm{~d}, J=8.5 \mathrm{~Hz}, 2 \mathrm{H}), 3.85$ (s, 3H); ${ }^{13} \mathrm{C}$ NMR (175 MHz, $\left.\mathrm{CDCl}_{3}\right): \delta 192.6,159.6,145.6,133.7,133.5,131.2,130.7,130.0$, 127.5, 127.3, 113.9, 55.3.

Spectral data of 4'-chloro-[1,1'-biphenyl]-4-carbaldehyde (5g-para):

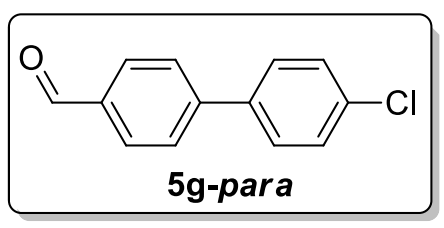

Compound (5g-para) was purified on silica gel column using hexane as the eluent; White solid (20.3 mg, $0.09 \mathrm{mmol}, 38 \%$ ); ${ }^{1} \mathrm{H}$ NMR (700 MHz, $\mathrm{CDCl}_{3}$ ): $\delta 10.03$ (s, 1H), 7.93 (d, $J=8.1 \mathrm{~Hz}$, 2H), $7.69(\mathrm{~d}, J=7.9 \mathrm{~Hz}, 2 \mathrm{H}), 7.55(\mathrm{~d}, J=8.4 \mathrm{~Hz}, 2 \mathrm{H}), 7.43(\mathrm{~d}, J=8.4 \mathrm{~Hz}, 2 \mathrm{H}) ;{ }^{13} \mathrm{C}$ NMR $(175$ $\mathrm{MHz}, \mathrm{CDCl}_{3}$ ): $\delta 191.7,145.8,138.1,135.3,134.2,130.3,129.2,128.5,127.5 ;$ HRMS (EI-MS) m/z: [M]+ calcd. For $\mathrm{C}_{13} \mathrm{H}_{9} \mathrm{ClO}: 216.0342$; found: 216.0339 .

Spectral data of 4'-chloro-[1,1'-biphenyl]-2-carbaldehyde (5g-ortho):

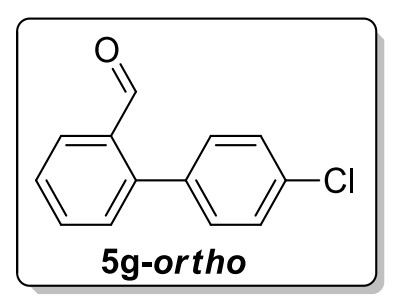

Compound (5g-ortho) was purified on silica gel column using hexane as the eluent; White solid (10.2 mg, $0.04 \mathrm{mmol}, 19 \%) ;{ }^{1} \mathrm{H}$ NMR (700 MHz, $\left.\mathrm{CDCl}_{3}\right): \delta 9.94(\mathrm{~s}, 1 \mathrm{H}), 8.01 \sim 7.99(\mathrm{~m}, 1 \mathrm{H})$, $7.63 \sim 7.61(\mathrm{~m}, 1 \mathrm{H}), 7.49(t, J=7.5 \mathrm{~Hz}, 1 \mathrm{H}), 7.43(\mathrm{~d}, J=8.3 \mathrm{~Hz}, 2 \mathrm{H}), 7.39 \sim 7.38(\mathrm{~m}, 1 \mathrm{H}), 7.30$ 
$(\mathrm{d}, J=8.4 \mathrm{~Hz}, 2 \mathrm{H}) ;{ }^{13} \mathrm{C} \mathrm{NMR}\left(175 \mathrm{MHz}, \mathrm{CDCl}_{3}\right): \delta 191.9,144.5,136.2,134.4,133.7,133.6$, $131.2,130.6,128.6,128.1,127.9$.

Spectral data of 4'-fluoro-[1,1'-biphenyl]-4-carbaldehyde (5h-para):

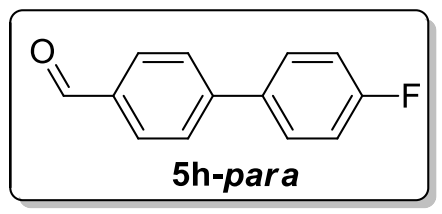

Compound (5h-para) was purified on silica gel column using hexane as the eluent; White solid (21.6 mg, $0.10 \mathrm{mmol}, 40 \%) ;{ }^{1} \mathrm{H} \mathrm{NMR}\left(700 \mathrm{MHz}, \mathrm{CDCl}_{3}\right): \delta 10.06$ (s, 1H), 7.97 (d, J=8.2 Hz, 2H), $7.74(\mathrm{~d}, J=8.1 \mathrm{~Hz}, 2 \mathrm{H}), 7.72(\mathrm{~m}, 4 \mathrm{H}) ;{ }^{13} \mathrm{C} \mathrm{NMR}\left(175 \mathrm{MHz}, \mathrm{CDCl}_{3}\right): \delta 191.7,145.5,143.24$, 143.23, 135.8, 130.3, 127.9, 127.7, 125.9; HRMS (EI-MS) m/z: [M]+ calcd. For $\mathrm{C}_{13} \mathrm{H}_{9} \mathrm{FO}_{2}$ : 200.0637; found: 200.0836 .

Spectral data of 4'-fluoro-[1,1'-biphenyl]-2-carbaldehyde (5h-ortho):

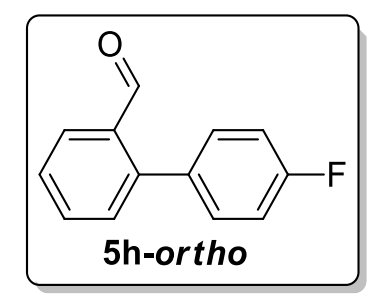

Compound (5h-ortho) was purified on silica gel column using hexane as the eluent; colorless solid (10.8 mg, $0.05 \mathrm{mmol}, 20 \%) ;{ }^{1} \mathrm{H} \mathrm{NMR}\left(700 \mathrm{MHz}, \mathrm{CDCl}_{3}\right): \delta 9.93(\mathrm{~s}, 1 \mathrm{H}), 8.03$ (d, J= $\left.7.2 \mathrm{~Hz}, 1 \mathrm{H}\right)$, $7.72(\mathrm{~d}, J=7.9 \mathrm{~Hz}, 2 \mathrm{H}), 7.67 \sim 7.64(\mathrm{~m}, 1 \mathrm{H}), 7.54(t, J=7.6 \mathrm{~Hz}, 1 \mathrm{H}), 7.49(\mathrm{~d}, J=7.9 \mathrm{~Hz}, 2 \mathrm{H})$, $7.41(\mathrm{~d}, J=7.3 \mathrm{~Hz}, 1 \mathrm{H}) ;{ }^{13} \mathrm{C} \mathrm{NMR}\left(175 \mathrm{MHz}, \mathrm{CDCl}_{3}\right): \delta 191.5,144.1,141.5,133.7,130.6,130.3$, $130.2,128.5,128.1,125.39,125.37$.

Spectral data of 3'-methyl-[1,1'-biphenyl]-4-carbaldehyde (5i-para):

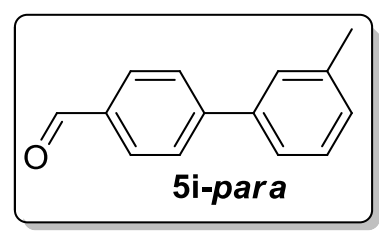

Compound (5i-para) was purified on silica gel column using hexane as the eluent; White solid (27 mg, 0.13 mmol, 50\%); ${ }^{1} \mathrm{H}$ NMR (700 MHz, $\left.\mathrm{CDCl}_{3}\right): \delta 10.02(\mathrm{~s}, 1 \mathrm{H}), 7.92(\mathrm{~d}, J=8.2 \mathrm{~Hz}, 2 \mathrm{H}), 7.72$ $(\mathrm{d}, J=8.1 \mathrm{~Hz}, 2 \mathrm{H}), 7.56 \sim 7.52(\mathrm{~m}, 2 \mathrm{H}), 7.27(\mathrm{~d}, J=7.7 \mathrm{~Hz}, 2 \mathrm{H}), 2.39(\mathrm{~s}, 3 \mathrm{H}) ;{ }^{13} \mathrm{C}$ NMR $(175$ $\left.\mathrm{MHz}, \mathrm{CDCl}_{3}\right): \delta 192.6,145.9,138.0,134.7,133.7,133.5,130.7,130.0,129.1,127.54,127.52$, 21.1; HRMS (EI-MS) m/z: [M]+ calcd. For $\mathrm{C}_{14} \mathrm{H}_{1} \mathrm{O}$ : 196.0888; found: 196.0886. 
Spectral data of 3'-methyl-[1,1'-biphenyl]-2-carbaldehyde (5i-ortho):

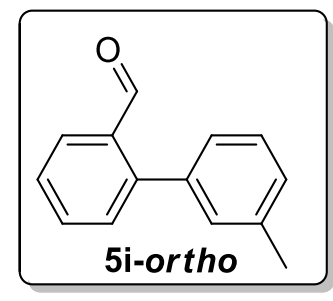

Compound (5i-ortho) was purified on silica gel column using hexane as the eluent; Yellow liquid (13.5 mg, $0.06 \mathrm{mmol}, 25 \%) ;{ }^{1} \mathrm{H}$ NMR (700 MHz, $\mathrm{CDCl}_{3}$ ): $\delta 9.97$ (s, $\left.1 \mathrm{H}\right), 8.0(\mathrm{~d}, J=7.7 \mathrm{~Hz}, 1 \mathrm{H})$, $7.61 \sim 7.58(\mathrm{~m}, 1 \mathrm{H}), 7.45(t, J=7.6 \mathrm{~Hz}, 1 \mathrm{H}), 7.42(\mathrm{~d}, J=7.7 \mathrm{~Hz}, 1 \mathrm{H}), 7.27 \sim 7.24(\mathrm{~m}, 4 \mathrm{H}), 2.41$ $(\mathrm{s}, 3 \mathrm{H}) ;{ }^{13} \mathrm{C} \mathrm{NMR}\left(175 \mathrm{MHz}, \mathrm{CDCl}_{3}\right): \delta 192.6,145.9,138.0,134.7,133.6,133.4,130.7,129.9$, $129.1,127.5,127.4,21.1$. two carbon merged with other peaks.

Spectral data of 3'-methoxy-[1,1'-biphenyl]-4-carbaldehyde (5j-para):

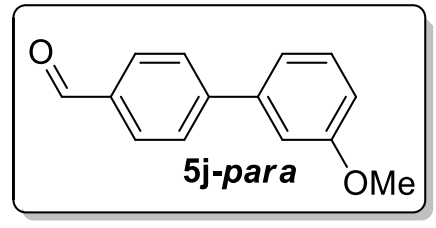

Compound (5j-para) was purified on silica gel column using hexane as the eluent; Yellow oil (30 mg, $0.14 \mathrm{mmol}, 55 \%) ;{ }^{1} \mathrm{H} \mathrm{NMR}$ (700 MHz, $\mathrm{CDCl}_{3}$ ): $\delta 10.03$ (s, $\left.1 \mathrm{H}\right), 7.93$ (d, $\left.J=8.1 \mathrm{~Hz}, 2 \mathrm{H}\right), 7.73$ $(\mathrm{d}, J=8.1 \mathrm{~Hz}, 2 \mathrm{H}), 7.37(t, J=7.9 \mathrm{~Hz}, 1 \mathrm{H}), 7.20(\mathrm{~d}, J=7.4 \mathrm{~Hz}, 1 \mathrm{H}), 7.13(\mathrm{~s}, 1 \mathrm{H}), 6.95 \sim 6.93(\mathrm{~m}$, 1H), 3.86 (s, 3H); $\left.{ }^{13} \mathrm{C} \mathrm{NMR} \mathrm{(175} \mathrm{MHz,} \mathrm{CDCl}_{3}\right): \delta 191.9,160.0,147.0,141.2,135.3,130.2,130.0$, 127.7, 119.8, 113.7, 113.1, 55.3; HRMS (EI-MS) m/z: [M]+ calcd. For $\mathrm{C}_{14} \mathrm{H}_{12} \mathrm{O}_{2}: 212.0837$; found: 212.0830 .

Spectral data of 3'-methoxy-[1,1'-biphenyl]-2-carbaldehyde (5j-ortho):

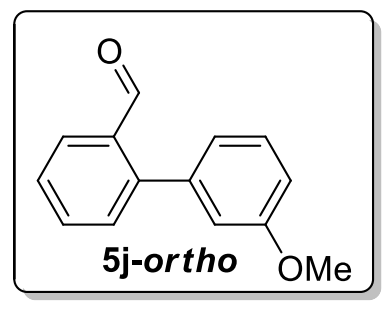

Compound (5j-ortho) was purified on silica gel column using hexane as the eluent; Colorless oil (14.5 mg, $0.06 \mathrm{mmol}, 27 \%) ;{ }^{1} \mathrm{H}$ NMR (700 MHz, $\mathrm{CDCl}_{3}$ ): $\delta 9.97$ (s, $\left.1 \mathrm{H}\right), 8.0(\mathrm{~d}, J=7.3 \mathrm{~Hz}, 1 \mathrm{H})$, $7.62 \sim 7.60(\mathrm{~m}, 1 \mathrm{H}), 7.47(t, J=7.6 \mathrm{~Hz}, 1 \mathrm{H}), 7.43(\mathrm{~d}, J=7.6 \mathrm{~Hz}, 1 \mathrm{H}), 7.35(t, J=7.8 \mathrm{~Hz}, 1 \mathrm{H})$, $6.97 \sim 6.95(\mathrm{~m}, 1 \mathrm{H}), 6.93(\mathrm{~d}, J=7.4 \mathrm{~Hz}, 1 \mathrm{H}), 6.90(\mathrm{~s}, 1 \mathrm{H}), 3.83(\mathrm{~s}, 3 \mathrm{H}) ;{ }^{13} \mathrm{C}$ NMR $(175 \mathrm{MHz}$, 
$\left.\mathrm{CDCl}_{3}\right): \delta 192.4,159.5,145.8,139.1,133.7,133.5,130.5,129.4,127.8,127.4,122.7,115.6,113.6$, 55.3 .

Spectral data of 3'-chloro-[1,1'-biphenyl]-4-carbaldehyde (5k-para):

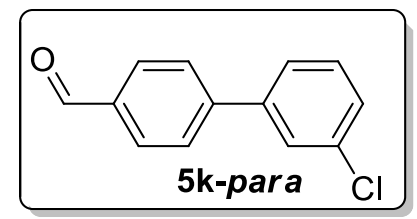

Compound (5k-para) was purified on silica gel column using hexane as the eluent; White solid (21.4 mg, $0.09 \mathrm{mmol}, 40 \%$ ); ${ }^{1} \mathrm{H}$ NMR (700 MHz, $\mathrm{CDCl}_{3}$ ): $\delta 10.03$ (s, $\left.1 \mathrm{H}\right), 7.94$ (d, $J=8.2 \mathrm{~Hz}$, $2 \mathrm{H}), 7.70(\mathrm{~d}, J=8.1 \mathrm{~Hz}, 2 \mathrm{H}), 7.59(\mathrm{~s}, 1 \mathrm{H}), 7.48(\mathrm{~d}, J=7.3 \mathrm{~Hz}, 1 \mathrm{H}), 7.40 \sim 7.36(\mathrm{~m}, 2 \mathrm{H}) ;{ }^{13} \mathrm{C}$ NMR $\left(175 \mathrm{MHz}, \mathrm{CDCl}_{3}\right): \delta 191.7,145.6,141.5,135.6,134.9,130.3,130.2,128.4,127.7,124.4,125.5$; HRMS (EI-MS) m/z: [M]+ calcd. For $\mathrm{C}_{13} \mathrm{H}_{9} \mathrm{ClO}: 216.0342$; found: 216.0336.

Spectral data of 3'-chloro-[1,1'-biphenyl]-2-carbaldehyde (5k-ortho):

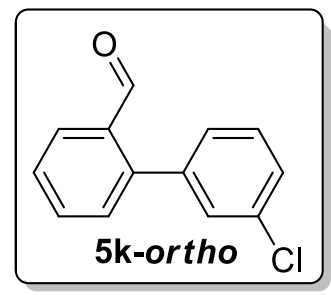

Compound (5k-ortho) was purified on silica gel column using hexane as the eluent; Colorless solid (18.7 mg, $0.08 \mathrm{mmol}, 35 \%) ;{ }^{1} \mathrm{H}$ NMR (700 MHz, $\left.\mathrm{CDCl}_{3}\right): \delta 9.95(\mathrm{~s}, 1 \mathrm{H}), 8.01 \sim 8.00(\mathrm{~m}$, 1H), $7.64 \sim 7.62(\mathrm{~m}, 1 \mathrm{H}), 7.50(t, J=7.5 \mathrm{~Hz}, 1 \mathrm{H}), 7.41 \sim 7.37(\mathrm{~m}, 4 \mathrm{H}), 7.22(\mathrm{~s}, 1 \mathrm{H}) ;{ }^{13} \mathrm{C}$ NMR $\left(175 \mathrm{MHz}, \mathrm{CDCl}_{3}\right): \delta 191.8,144.2,139.6,134.5,133.7,133.6,130.6,129.8,129.6,128.35,128.32$, 128.2, 127.8 .

Spectral data of 2'-methyl-[1,1'-biphenyl]-4-carbaldehyde (5l-para):

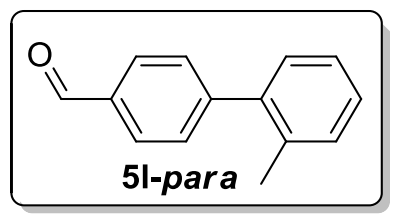

Compound (5l-para) was purified on silica gel column using hexane as the eluent; Colorless oil (22.1 mg, $0.11 \mathrm{mmol}, 41 \%) ;{ }^{1} \mathrm{H}$ NMR (700 MHz, $\left.\mathrm{CDCl}_{3}\right): \delta 10.03$ (s, $\left.1 \mathrm{H}\right), 7.92(\mathrm{~d}, J=7.9 \mathrm{~Hz}$, 2H), $7.73(\mathrm{~d}, J=8.0 \mathrm{~Hz}, 2 \mathrm{H}), 7.43 \sim 7.41(\mathrm{~m}, 2 \mathrm{H}), 7.35(t, J=7.5 \mathrm{~Hz}, 1 \mathrm{H}), 7.21(\mathrm{~d}, J=7.4 \mathrm{~Hz}$, 1H), 2.42 (s, 3H); ${ }^{13} \mathrm{C}$ NMR (175 MHz, $\left.\mathrm{CDCl}_{3}\right): \delta 191.9,147.3,139.7,138.6,135.1,130.2,129.2$, 
128.9, 128.1, 127.6, 124.4, 21.5; HRMS (EI-MS) m/z: [M]+ calcd. For $\mathrm{C}_{14} \mathrm{H}_{12} \mathrm{O}$ : 196.0888; found: 196.0884.

Spectral data of 2'-methyl-[1,1'-biphenyl]-2-carbaldehyde (5l-ortho):

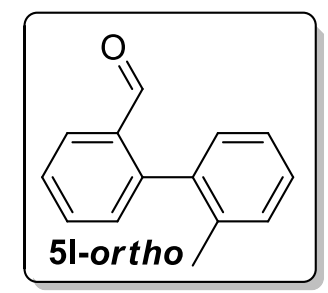

Compound (5l-ortho) was purified on silica gel column using hexane as the eluent; Colorless liquid (17.8 mg, $0.09 \mathrm{mmol}, 33 \%)$; ${ }^{1} \mathrm{H} \mathrm{NMR}\left(700 \mathrm{MHz}, \mathrm{CDCl}_{3}\right): \delta 9.96$ (s, 1H), 8.0 (d, J = 7.7 Hz, 1H), $7.60(t, J=7.4 \mathrm{~Hz}, 1 \mathrm{H}), 7.46(t, J=7.5 \mathrm{~Hz}, 1 \mathrm{H}), 7.42(\mathrm{~d}, J=7.6 \mathrm{~Hz}, 1 \mathrm{H}), 7.33(t, J=7.4 \mathrm{~Hz}$, 1H), $7.23 \sim 7.22(\mathrm{~m}, 1 \mathrm{H}), 7.17 \sim 7.15(\mathrm{~m}, 2 \mathrm{H}), 2.40(\mathrm{~s}, 3 \mathrm{H}) ;{ }^{13} \mathrm{C} \mathrm{NMR}\left(175 \mathrm{MHz}, \mathrm{CDCl}_{3}\right): \delta 192.6$, 146.1, 138.1, 137.6, 133.7, 133.4, 130.8, 130.7, 128.8, 128.2, 127.6, 127.4, 127.2, 21.4.

Spectral data of 2'-methoxy-[1,1'-biphenyl]-4-carbaldehyde (5m-para):

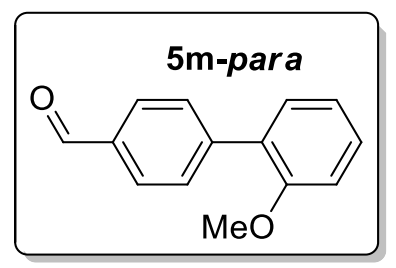

Compound (5m-para) was purified on silica gel column using hexane as the eluent; White solid (24.6 mg, $0.11 \mathrm{mmol}, 46 \%) ;{ }^{1} \mathrm{H} \mathrm{NMR}\left(700 \mathrm{MHz}, \mathrm{CDCl}_{3}\right): \delta 10.04$ (s, 1H), 7.90 (d, J=7.8 Hz, 2H), $7.69(\mathrm{~d}, J=7.8 \mathrm{~Hz}, 2 \mathrm{H}), 7.38 \sim 7.33(\mathrm{~m}, 2 \mathrm{H}), 7.05(t, J=7.4 \mathrm{~Hz}, 1 \mathrm{H}), 7.0(\mathrm{~d}, J=8.1 \mathrm{~Hz}, 1 \mathrm{H})$, $3.82(\mathrm{~s}, 3 \mathrm{H}) ;{ }^{13} \mathrm{C} \mathrm{NMR}\left(175 \mathrm{MHz}, \mathrm{CDCl}_{3}\right): \delta 192.0,156.4,145.0,134.8,130.7,130.1,129.6,129.4$, 129.2, 120.9, 111.3, 55.5; HRMS (EI-MS) m/z: [M]+ calcd. For $\mathrm{C}_{14} \mathrm{H}_{12} \mathrm{O}_{2}$ : 212.0837; found: 212.0835 .

\section{Spectral data of 2'-methoxy-[1,1'-biphenyl]-2-carbaldehyde (5m-ortho):}

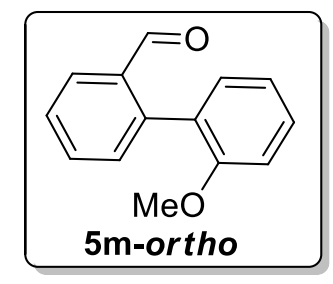

Compound (5m-ortho) was purified on silica gel column using hexane as the eluent; White solid (12.3 mg, $0.05 \mathrm{mmol}, 23 \%) ;{ }^{1} \mathrm{H}$ NMR (700 MHz, $\left.\mathrm{CDCl}_{3}\right): \delta 9.76(\mathrm{~s}, 1 \mathrm{H}), 7.98(\mathrm{~d}, J=7.7 \mathrm{~Hz}, 1 \mathrm{H})$, 
$7.62(t, J=7.4 \mathrm{~Hz}, 1 \mathrm{H}), 7.46(t, J=7.4 \mathrm{~Hz}, 1 \mathrm{H}), 7.40(t, J=7.8 \mathrm{~Hz}, 1 \mathrm{H}), 7.34(\mathrm{~d}, J=7.5 \mathrm{~Hz}, 1 \mathrm{H})$, $7.26(\mathrm{~d}, J=7.3 \mathrm{~Hz}, 1 \mathrm{H}), 7.06(t, J=7.3 \mathrm{~Hz}, 1 \mathrm{H}), 6.95(\mathrm{~d}, J=8.2 \mathrm{~Hz}, 1 \mathrm{H}), 3.72(\mathrm{~s}, 3 \mathrm{H}) ;{ }^{13} \mathrm{C} \mathrm{NMR}$ $\left(175 \mathrm{MHz}, \mathrm{CDCl}_{3}\right): \delta 192.6,156.4,141.8,133.9,133.7,131.4,131.2,129.6,127.7,126.8,126.6$, 120.9, 110.6, 55.3.

Spectral data of 2'-chloro-[1,1'-biphenyl]-4-carbaldehyde (5n-para):

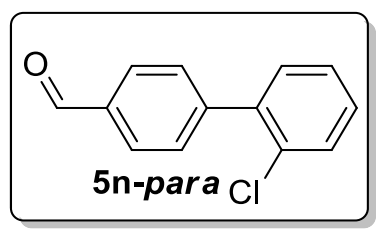

Compound (5n-para) was purified on silica gel column using hexane as the eluent; Yellow oil

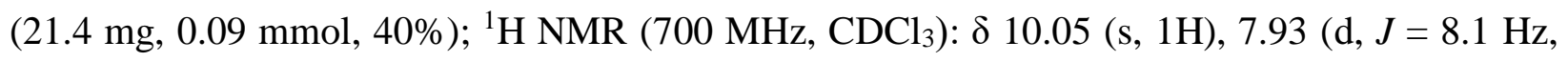
2H), $7.60(\mathrm{~d}, J=8.0 \mathrm{~Hz}, 2 \mathrm{H}), 7.48 \sim 7.47(\mathrm{~m}, 1 \mathrm{H}), 7.33 \sim 7.30(\mathrm{~m}, 3 \mathrm{H}) ;{ }^{13} \mathrm{C}$ NMR $(175 \mathrm{MHz}$, $\mathrm{CDCl}_{3}$ ): $\delta 191.9,145.4,139.2,135.4,132.2,131.0,130.2,129.4,129.3,128.7,127.0$; HRMS (EIMS) m/z: [M]+ calcd. For $\mathrm{C}_{13} \mathrm{H}_{9} \mathrm{ClO}: 216.0342$; found: 216.0339 .

Spectral data of 2'-chloro-[1,1'-biphenyl]-2-carbaldehyde (5n-ortho):

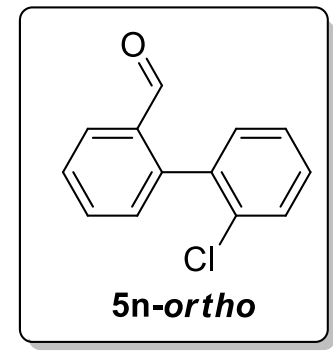

Compound (5n-ortho) was purified on silica gel column using hexane as the eluent; White solid (10.7 mg, $0.04 \mathrm{mmol}, 20 \%) ;{ }^{1} \mathrm{H}$ NMR $\left(700 \mathrm{MHz}, \mathrm{CDCl}_{3}\right): \delta 9.77(\mathrm{~s}, 1 \mathrm{H}), 8.01(\mathrm{~d}, J=7.7 \mathrm{~Hz}, 1 \mathrm{H})$, $7.65 \sim 7.63(\mathrm{~m}, 1 \mathrm{H}), 7.53(t, J=7.6 \mathrm{~Hz}, 1 \mathrm{H}), 7.49 \sim 7.47(\mathrm{~m}, 1 \mathrm{H}), 7.38 \sim 7.30(\mathrm{~m}, 4 \mathrm{H}) ;{ }^{13} \mathrm{C} \mathrm{NMR}$ $\left(175 \mathrm{MHz}, \mathrm{CDCl}_{3}\right): \delta 191.5,142.6,136.8,133.7,133.6,133.5,131.6,130.9,129.6,129.5,128.5$, 127.3, 126.8 .

Spectral data of 4-(naphthalen-1-yl)benzaldehyde (5o-para):

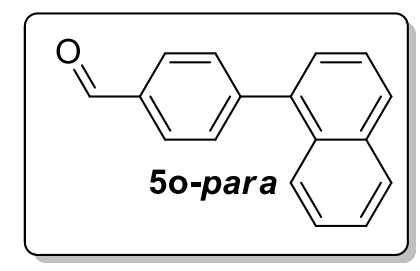


Compound (5o-para) was purified on silica gel column using hexane as the eluent; Yellow solid (21.3 mg, $0.09 \mathrm{mmol}, 40 \%) ;{ }^{1} \mathrm{H} \mathrm{NMR}\left(700 \mathrm{MHz}, \mathrm{CDCl}_{3}\right): \delta 10.10(\mathrm{~s}, 1 \mathrm{H}), 8.0(\mathrm{~d}, J=7.7 \mathrm{~Hz}, 2 \mathrm{H})$, $7.92 \sim 7.89(\mathrm{~m}, 2 \mathrm{H}), 7.81(\mathrm{~d}, J=8.4 \mathrm{~Hz}, 1 \mathrm{H}), 7.66(\mathrm{~d}, J=7.9 \mathrm{~Hz}, 2 \mathrm{H}), 7.54 \sim 7.49(\mathrm{~m}, 2 \mathrm{H}), 7.45$ $\sim 7.41(\mathrm{~m}, 2 \mathrm{H}) ;{ }^{13} \mathrm{C} \mathrm{NMR}\left(175 \mathrm{MHz}, \mathrm{CDCl}_{3}\right): \delta 192.0,147.2,138.7,135.2,133.7,131.0,130.7$, 129.7, 128.5, 128.4, 126.9, 126.4, 126.0, 125.4, 125.3; HRMS (EI-MS) m/z: [M]+ calcd. For $\mathrm{C}_{17} \mathrm{H}_{12} \mathrm{O}: 232.0888$; found: 232.0887.

Spectral data of 2-(naphthalen-1-yl)benzaldehyde (5o-ortho):

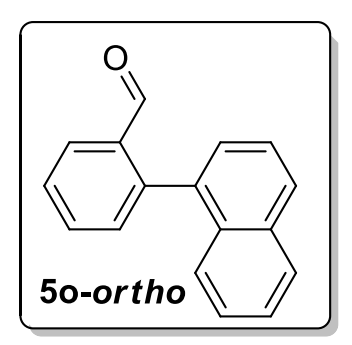

Compound (5o-ortho) was purified on silica gel column using hexane as the eluent; White solid (14.4 mg, $0.06 \mathrm{mmol}, 27 \%) ;{ }^{1} \mathrm{H}$ NMR $\left(700 \mathrm{MHz}, \mathrm{CDCl}_{3}\right): \delta 9.60(\mathrm{~s}, 1 \mathrm{H}), 8.0(\mathrm{~d}, J=7.8 \mathrm{~Hz}, 1 \mathrm{H})$, $7.93 \sim 7.91(\mathrm{~m}, 2 \mathrm{H}), 7.68(t, J=7.4 \mathrm{~Hz}, 1 \mathrm{H}), 7.58 \sim 7.52(\mathrm{~m}, 2 \mathrm{H}), 7.50 \sim 7.43(\mathrm{~m}, 3 \mathrm{H}), 7.41 \sim$ 7.39 (m, 2H); ${ }^{13} \mathrm{C}$ NMR (175 MHz, $\left.\mathrm{CDCl}_{3}\right): \delta$ 192.1, 144.2, 135.4, 134.8, 133.6, 133.3, 132.7, $131.7,128.6,128.3,128.1,127.0,126.7,126.1,125.7,125.0$, one carbon merged with other peaks.

Spectral data of 4-(thiophen-2-yl)benzaldehyde (3p-para):

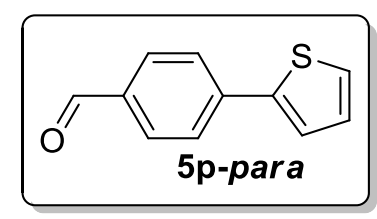

Compound (5p-para) was purified on silica gel column using hexane as the eluent; Pale yellow

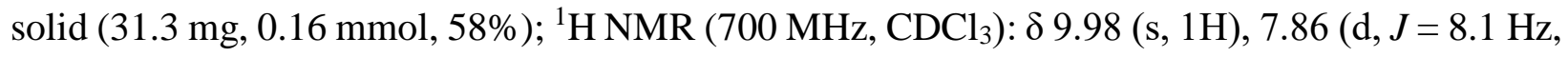
2H), $7.74(\mathrm{~d}, J=8.1 \mathrm{~Hz}, 2 \mathrm{H}), 7.45 \sim 7.44(\mathrm{~m}, 1 \mathrm{H}), 7.38 \sim 7.37(\mathrm{~m}, 1 \mathrm{H}), 7.12 \sim 7.10(\mathrm{~m}, 1 \mathrm{H}) ;{ }^{13} \mathrm{C}$ NMR (175 MHz, $\left.\mathrm{CDCl}_{3}\right): \delta 191.4,142.7,140.0,135.0,130.4,128.4,126.9,126.0,125.0 ;$ HRMS (EI-MS) m/z: [M]+ calcd. For $\mathrm{C}_{11} \mathrm{H}_{8} \mathrm{OS}: 188.0296$; found: 188.0294 .

Spectral data of 2-(thiophen-2-yl)benzaldehyde (5p-ortho): 


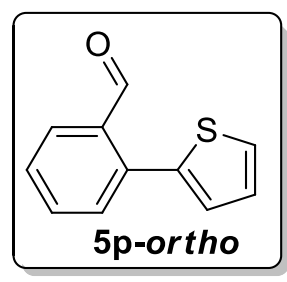

Compound (5p-ortho) was purified on silica gel column using hexane as the eluent; Pale yellow oil (15.7 mg, $0.08 \mathrm{mmol}, 29 \%)$; ${ }^{1} \mathrm{H} \mathrm{NMR}$ (700 MHz, $\left.\mathrm{CDCl}_{3}\right)$ : $\delta 10.16(\mathrm{~s}, 1 \mathrm{H}), 7.99 \sim 7.98(\mathrm{~m}, 1 \mathrm{H})$, $7.61 \sim 7.58(\mathrm{~m}, 1 \mathrm{H}), 7.53 \sim 7.52(\mathrm{~m}, 1 \mathrm{H}), 7.48 \sim 7.44(\mathrm{~m}, 2 \mathrm{H}), 7.13 \sim 7.12(\mathrm{~m}, 1 \mathrm{H}), 7.06 \sim 7.05$ (m, 1H); $\left.{ }^{13} \mathrm{C} \mathrm{NMR} \mathrm{(175} \mathrm{MHz,} \mathrm{CDCl}_{3}\right): \delta 192.0,138.7,138.0,134.1,133.5,131.2,129.5,128.2$, 127.8, 127.7, 127.3.

Spectral data of 4-(4-methylthiophen-2-yl)benzaldehyde (3q-para):

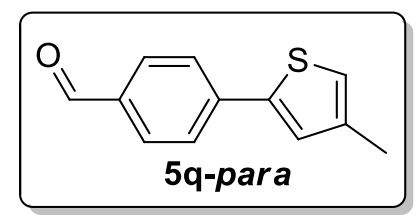

Compound (5q-para) was purified on silica gel column using hexane as the eluent; Yellow solid (26.9 mg, $0.13 \mathrm{mmol}, 50 \%) ;{ }^{1} \mathrm{H} \mathrm{NMR}\left(700 \mathrm{MHz}, \mathrm{CDCl}_{3}\right): \delta 9.96(\mathrm{~s}, 1 \mathrm{H}), 7.85$ (d, $\left.J=8.4 \mathrm{~Hz}, 2 \mathrm{H}\right)$, $7.70(\mathrm{~d}, J=7.0 \mathrm{~Hz}, 2 \mathrm{H}), 7.25$ (s, 1H), $6.95(\mathrm{~s}, 1 \mathrm{H}), 2.28$ (s, 3H); ${ }^{13} \mathrm{C} \mathrm{NMR}\left(175 \mathrm{MHz}, \mathrm{CDCl}_{3}\right): \delta$ 191.4, 142.3, 140.2, 139.1, 134.9, 130.4, 127.3, 125.7, 122.4, 15.7; HRMS (EI-MS) m/z: [M]+ calcd. For $\mathrm{C}_{12} \mathrm{H}_{10} \mathrm{OS}: 202.0452$; found: 202.0447.

Spectral data of 2-(4-methylthiophen-2-yl)benzaldehyde (3q-ortho):

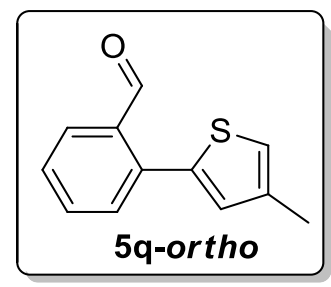

Compound (5q-ortho) was purified on silica gel column using hexane as the eluent; Yellow oil (13.4 mg, 0.0.06 mmol, 25\%); ${ }^{1} \mathrm{H}$ NMR (700 MHz, $\left.\mathrm{CDCl}_{3}\right): \delta 10.18$ (s, $\left.1 \mathrm{H}\right), 7.58(t, J=7.4 \mathrm{~Hz}$, 1H), $7.70(\mathrm{~d}, J=7.7 \mathrm{~Hz}, 2 \mathrm{H}), 7.44(t, J=7.5 \mathrm{~Hz}, 1 \mathrm{H}), 7.01(\mathrm{~s}, 1 \mathrm{H}), 6.85(\mathrm{~s}, 1 \mathrm{H}), 2.29(\mathrm{~s}, 3 \mathrm{H}) ;{ }^{13} \mathrm{C}$ NMR (175 MHz, $\left.\mathrm{CDCl}_{3}\right): \delta 192.2,138.5,138.4,138.3,134.0,133.5,131.9,131.0,128.0,127.7$, 122.7, 15.7 .

Spectral data of 4-(furan-2-yl)benzaldehyde (5r-para): 


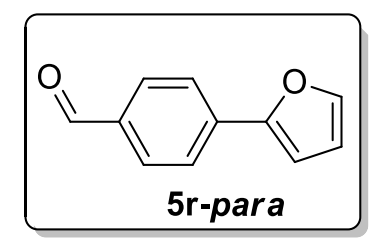

Compound (5r-para) was purified on silica gel column using hexane as the eluent; Yellow solid (19.6 mg, $0.11 \mathrm{mmol}, 42 \%) ;{ }^{1} \mathrm{H}$ NMR $\left(700 \mathrm{MHz}, \mathrm{CDCl}_{3}\right): \delta 9.97(\mathrm{~s}, 1 \mathrm{H}), 7.87(\mathrm{~d}, J=8.2 \mathrm{~Hz}, 2 \mathrm{H})$, $7.79(\mathrm{~d}, J=8.2 \mathrm{~Hz}, 2 \mathrm{H}), 7.53(\mathrm{~d}, J=1.0 \mathrm{~Hz}, 1 \mathrm{H}), 6.82(\mathrm{~d}, J=3.2 \mathrm{~Hz}, 1 \mathrm{H}), 6.51(\mathrm{dd}, J=3.2,1.6$ $\mathrm{Hz}, 1 \mathrm{H}) ;{ }^{13} \mathrm{C} \mathrm{NMR}\left(175 \mathrm{MHz}, \mathrm{CDCl}_{3}\right): \delta$ 191.5, 152.6, 143.6, 136.0, 134.9, 130.3, 123.9, 112.2 , 108.1; HRMS (EI-MS) m/z: [M]+ calcd. For $\mathrm{C}_{11} \mathrm{H}_{8} \mathrm{O}_{2}: 172.0524$; found: 172.0521.

Spectral data of 2-(furan-2-yl)benzaldehyde (5r-ortho):

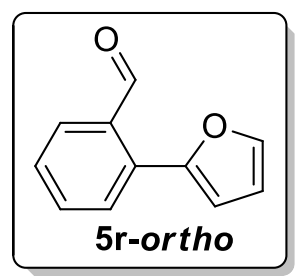

Compound (5r-ortho) was purified on silica gel column using hexane as the eluent; Yellow oil (8.4 mg, $0.04 \mathrm{mmol}, 18 \%) ;{ }^{1} \mathrm{H}$ NMR (700 MHz, $\left.\mathrm{CDCl}_{3}\right): \delta 10.36(\mathrm{~s}, 1 \mathrm{H}), 7.96 \sim 7.95(\mathrm{~m}, 1 \mathrm{H})$, $7.67(\mathrm{~d}, J=7.7 \mathrm{~Hz}, 1 \mathrm{H}), 7.61 \sim 7.58(\mathrm{~m}, 2 \mathrm{H}), 7.42(t, J=7.4 \mathrm{~Hz}, 1 \mathrm{H}), 6.61(\mathrm{~d}, J=3.0 \mathrm{~Hz}, 1 \mathrm{H})$, $6.54(\mathrm{dd}, J=3.1,1.5 \mathrm{~Hz}, 1 \mathrm{H}) ;{ }^{13} \mathrm{C} \mathrm{NMR}\left(175 \mathrm{MHz}, \mathrm{CDCl}_{3}\right): \delta 192.4,151.0,143.9,133.5,133.3$, 133.0, 128.3, 128.0, 127.9, 111.9, 111.2.

\section{Spectral data of 4-methylbenzaldehyde (5s-para):}

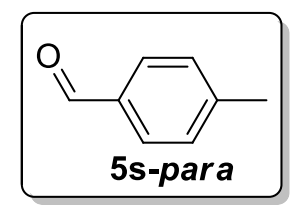

Compound (5s-para) was purified on silica gel column using hexane as the eluent; Colorless oil (17 mg, $\left.0.14 \mathrm{mmol}, 30 \%) ;{ }^{1} \mathrm{H} \mathrm{NMR} \mathrm{(400} \mathrm{MHz,} \mathrm{CDCl}_{3}\right): \delta 9.94$ (s, 1H), $7.75(\mathrm{~d}, J=8.0 \mathrm{~Hz}, 2 \mathrm{H}$ ), $7.30(\mathrm{~d}, J=7.9 \mathrm{~Hz}, 2 \mathrm{H}), 2.41$ (s, 3H); ${ }^{13} \mathrm{C}$ NMR $\left(175 \mathrm{MHz}, \mathrm{CDCl}_{3}\right): \delta 192.0,145.5,134.1,129.7$, 129.1, 21.8; HRMS (EI-MS) m/z: [M]+ calcd. For $\mathrm{C}_{8} \mathrm{H}_{8} \mathrm{O}$ : 120.0575; found: 120.0570 .

Spectral data 2-methylbenzaldehyde (5s-ortho): 


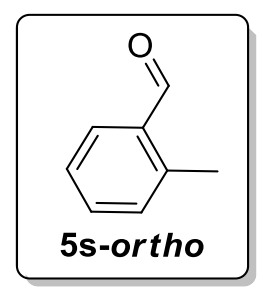

Compound (5s-ortho) was purified on silica gel column using hexane as the eluent; Colorless oil (14.1 mg, $0.11 \mathrm{mmol}, 25 \%) ;{ }^{1} \mathrm{H} \mathrm{NMR}\left(400 \mathrm{MHz}, \mathrm{CDCl}_{3}\right): \delta 10.25$ (s, 1H), 7.77 (d, J=8.7 Hz, 1H), $7.47 \sim 7.43(\mathrm{~m}, 1 \mathrm{H}), 7.34(t, J=7.5 \mathrm{~Hz}, 1 \mathrm{H}), 7.24(\mathrm{~d}, J=8.0 \mathrm{~Hz}, 1 \mathrm{H}), 2.65(\mathrm{~s}, 3 \mathrm{H}) ;{ }^{13} \mathrm{C} \mathrm{NMR}$ $\left(100 \mathrm{MHz}, \mathrm{CDCl}_{3}\right): \delta 192.6,140.5,134.1,133.6,132.0,131.7,126.2,19.5$.

Spectral data of 3-methylbenzaldehyde (5s-meta):

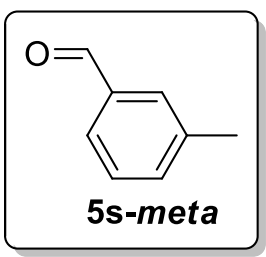

Compound (5s-meta) was purified on silica gel column using hexane as the eluent; Colorless oil (13 mg, $0.10 \mathrm{mmol}, 23 \%) ;{ }^{1} \mathrm{H} \mathrm{NMR}\left(400 \mathrm{MHz}, \mathrm{CDCl}_{3}\right): \delta 9.96(\mathrm{~s}, 1 \mathrm{H}), 7.67 \sim 7.65$ (m, 2H), 7.42 $\sim 7.39(\mathrm{~m}, 2 \mathrm{H}), 2.41(\mathrm{~s}, 3 \mathrm{H}) ;{ }^{13} \mathrm{C} \mathrm{NMR}\left(100 \mathrm{MHz}, \mathrm{CDCl}_{3}\right): \delta$ 192.6, 138.8, 136.4, 135.3, 130.0, 128.8, 127.2, 21.2.

Spectral data of 4-cyclopropylbenzaldehyde (5t-para):

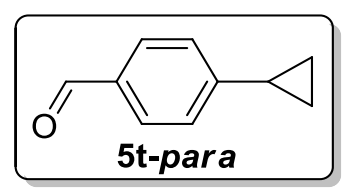

Compound (5t-para) was purified on silica gel column using hexane as the eluent; Yellow liquid (17.9 mg, $0.12 \mathrm{mmol}, 32 \%) ;{ }^{1} \mathrm{H} \mathrm{NMR}\left(700 \mathrm{MHz} \mathrm{CDCl}_{3}\right): \delta 9.91(\mathrm{~s}, 1 \mathrm{H}), 7.74(\mathrm{~d}, J=8.0 \mathrm{~Hz}, 2 \mathrm{H})$, $7.16(\mathrm{~d}, J=8.0 \mathrm{~Hz}, 2 \mathrm{H}), 1.95 \sim 1.93(\mathrm{~m}, 1 \mathrm{H}), 1.08 \sim 1.06(\mathrm{~m}, 2 \mathrm{H}), 0.79 \sim 0.78(\mathrm{~m}, 2 \mathrm{H}) ;{ }^{13} \mathrm{C} \mathrm{NMR}$ $\left(175 \mathrm{MHz}, \mathrm{CDCl}_{3}\right): \delta 191.8,152.1,134.0,129.8,125.8,15.9,10.6$; HRMS (EI-MS) m/z: [M]+ calcd. For $\mathrm{C}_{10} \mathrm{H}_{10} \mathrm{O}$ : 146.0732; found: 146.0729 .

Spectral data of 2-cyclopropylbenzaldehyde and 3-cyclopropylbenzaldehyde (5t-ortho:5tmeta): 


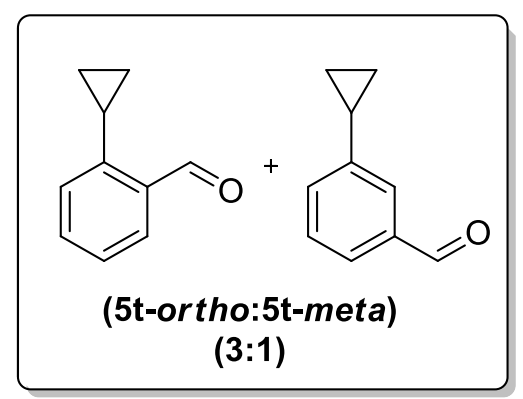

Compound (5t-ortho:5t-meta) was purified on silica gel column using hexane as the eluent; Colorless oil (23 mg, $0.15 \mathrm{mmol}, 41 \%) ;{ }^{1} \mathrm{H}$ NMR for major ortho isomer $\left(700 \mathrm{MHz}, \mathrm{CDCl}_{3}\right): \delta$ $10.28(\mathrm{~s}, 1 \mathrm{H}), 7.80 \sim 7.79(\mathrm{~m}, 1 \mathrm{H}), 7.47 \sim 7.45(\mathrm{~m}, 1 \mathrm{H}), 7.29(t, J=7.4 \mathrm{~Hz}, 1 \mathrm{H}), 7.10(\mathrm{~d}, J=7.7$ $\mathrm{Hz}, 1 \mathrm{H}), 2.62 \sim 2.59(\mathrm{~m}, 1 \mathrm{H}), 1.07 \sim 1.05(\mathrm{~m}, 2 \mathrm{H}), 0.77 \sim 0.75(\mathrm{~m}, 2 \mathrm{H}) ;{ }^{1} \mathrm{H}$ NMR for minor meta isomer: $\delta 9.95(\mathrm{~s}, 1 \mathrm{H}), 7.62(\mathrm{~d}, J=7.4 \mathrm{~Hz}, 1 \mathrm{H}), 7.54(\mathrm{~s}, 1 \mathrm{H}), 7.39(t, J=7.5 \mathrm{~Hz}, 1 \mathrm{H}), 7.32(\mathrm{~d}, J=$ $7.7 \mathrm{~Hz}, 1 \mathrm{H}), 1.96 \sim 1.94(\mathrm{~m}, 1 \mathrm{H}), 1.02 \sim 0.99(\mathrm{~m}, 2 \mathrm{H}), 0.74 \sim 0.72(\mathrm{~m}, 2 \mathrm{H}) ;{ }^{13} \mathrm{C}$ NMR for major ortho isomer $\left(175 \mathrm{MHz}, \mathrm{CDCl}_{3}\right): \delta 192.5,146.0,133.9,132.0,130.0,126.5,126.0,11.7,8.4 ;{ }^{13} \mathrm{C}$ NMR for minor meta isomer $\left(175 \mathrm{MHz}, \mathrm{CDCl}_{3}\right)$ : $\delta 193.5,145.2,136.4,134.8,128.8,127.2,126.2$, $15.1,9.5$.

\section{Spectral data of (3S,5S)-5-ethoxy-3-methyl-2-phenylisoxazolidine (8a):}

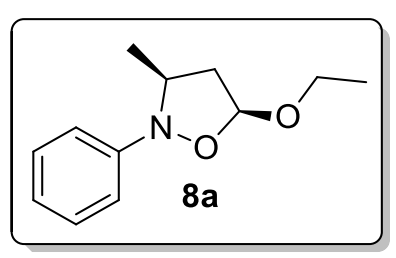

Compound 8a was purified on silica gel column using ethyl acetate/hexane: (10 : 90) as the eluent; Colorless oil (52.3 mg, $0.25 \mathrm{mmol}, 54 \%) ;{ }^{1} \mathrm{H}$ NMR (700 MHz, $\mathrm{CDCl}_{3}$ ): $\delta 7.28 \sim 7.25$ (m, 2H), $7.15 \sim 7.13(\mathrm{~m}, 2 \mathrm{H}), 7.03 \sim 7.01(\mathrm{~m}, 1 \mathrm{H}), 5.32(\mathrm{dd}, J=6.3,2.2 \mathrm{~Hz}, 1 \mathrm{H}), 3.93 \sim 3.87(\mathrm{~m}, 1 \mathrm{H}), 3.57$ $3.51(\mathrm{~m}, 1 \mathrm{H}), 3.45 \sim 3.42(\mathrm{~m}, 1 \mathrm{H}), 2.61 \sim 2.57(\mathrm{~m}, 1 \mathrm{H}), 2.01 \sim 1.98(\mathrm{~m}, 1 \mathrm{H}), 1.38(\mathrm{~d}, J=6.4 \mathrm{~Hz}$, $3 \mathrm{H}), 1.24(t, J=7.0 \mathrm{~Hz}, 3 \mathrm{H}) ;{ }^{13} \mathrm{C} \mathrm{NMR}\left(175 \mathrm{MHz}, \mathrm{CDCl}_{3}\right): \delta 150.5,128.6,123.4,117.8,101.4$, 64.0, 62.0, 43.1, 19.4, 15.1; HRMS (ESI-TOF) m/z: $[\mathrm{M}+\mathrm{H}]^{+}$calcd. For $\mathrm{C}_{12} \mathrm{H}_{17} \mathrm{NO}_{2}$ : 208.0840; found: 208.0840 .

Spectral data of (3S,5R)-5-ethoxy-3-methyl-2-phenylisoxazolidine (8a'): 


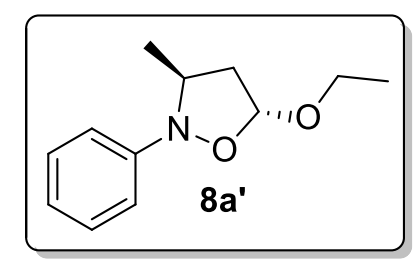

Compound 8a' was purified on silica gel column using ethyl acetate/hexane: (10:90) as the eluent; Colorless oil (19.4 mg, $0.09 \mathrm{mmol}, 18 \%) ;{ }^{1} \mathrm{H}$ NMR (700 MHz, $\mathrm{CDCl}_{3}$ ): $\delta 7.25 \sim 7.23$ (m, 2H), $7.04 \sim 7.03(\mathrm{~d}, J=7.8 \mathrm{~Hz}, 2 \mathrm{H}), 6.93(t, J=7.3 \mathrm{~Hz}, 1 \mathrm{H}), 5.26(\mathrm{~d}, J=5.1 \mathrm{~Hz}, 1 \mathrm{H}), 3.94 \sim 3.91(\mathrm{~m}$, $1 \mathrm{H}), 3.80 \sim 3.78(\mathrm{~m}, 1 \mathrm{H}), 3.51 \sim 3.49(\mathrm{~m}, 1 \mathrm{H}), 2.47 \sim 2.44(\mathrm{~m}, 1 \mathrm{H}), 2.20 \sim 1.17(\mathrm{~m}, 1 \mathrm{H}), 1.32(\mathrm{~d}$, $J=6.3 \mathrm{~Hz}, 3 \mathrm{H}), 1.05(t, J=7.0 \mathrm{~Hz}, 3 \mathrm{H}) ;{ }^{13} \mathrm{C} \mathrm{NMR}\left(175 \mathrm{MHz}, \mathrm{CDCl}_{3}\right): \delta 151.8,128.5,121.5$, $115.3,102.3,63.5,58.9,44.1,19.9,14.8$.

Spectral data of (3S,5S)-5-butoxy-3-methyl-2-phenylisoxazolidine (8b):

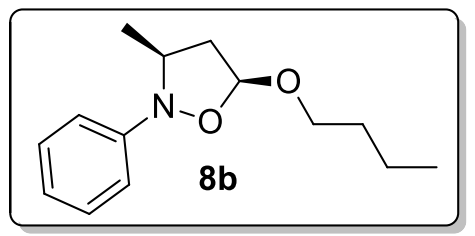

Compound $\mathbf{8 b}$ was purified on silica gel column using ethyl acetate/hexane: (10 : 90) as the eluent; Colorless liquid (60.3 mg, $0.25 \mathrm{mmol}, 55 \%) ;{ }^{1} \mathrm{H} \mathrm{NMR}\left(700 \mathrm{MHz}, \mathrm{CDCl}_{3}\right): \delta 7.26(t, J=8.0 \mathrm{~Hz}$, $2 \mathrm{H}), 7.12(\mathrm{~d}, J=7.9 \mathrm{~Hz}, 2 \mathrm{H}), 7.01(t, J=7.3 \mathrm{~Hz}, 1 \mathrm{H}), 5.31(\mathrm{dd}, J=6.2,1.7 \mathrm{~Hz}, 1 \mathrm{H}), 3.86 \sim 3.82$ (m, 1H), $3.48 \sim 3.44(\mathrm{~m}, 1 \mathrm{H}), 2.58 \sim 2.54(\mathrm{~m}, 1 \mathrm{H}), 2.0 \sim 1.98(\mathrm{~m}, 1 \mathrm{H}), 1.62 \sim 1.56(\mathrm{~m}, 1 \mathrm{H}), 1.38$ $(\mathrm{d}, J=6.5 \mathrm{~Hz}, 3 \mathrm{H}), 0.91(t, J=7.4 \mathrm{~Hz}, 3 \mathrm{H}) ;{ }^{13} \mathrm{C} \mathrm{NMR}\left(175 \mathrm{MHz}, \mathrm{CDCl}_{3}\right): \delta 150.6,128.6,123.3$, 117.6, 101.7, 68.5, 61.9, 42.8, 31.7, 19.5, 19.3, 13.8; HRMS (ESI-TOF) m/z: [M+H] ${ }^{+}$calcd. For $\mathrm{C}_{14} \mathrm{H}_{21} \mathrm{NO}_{2}$ : 236.1650; found: 236.1651.

\section{Spectral data of (3S,5R)-5-butoxy-3-methyl-2-phenylisoxazolidine (8b'):}

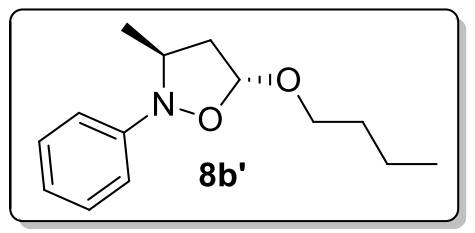


Compound 8b' was purified on silica gel column using ethyl acetate/hexane: $(9: 91)$ as the eluent; Colorless liquid (19.9 mg, $0.08 \mathrm{mmol}, 19 \%) ;{ }^{1} \mathrm{H}$ NMR (700 MHz, $\left.\mathrm{CDCl}_{3}\right): \delta 7.24 \sim 7.22(\mathrm{~m}, 2 \mathrm{H})$, $7.03 \sim 7.02(\mathrm{~d}, J=8.0 \mathrm{~Hz}, 2 \mathrm{H}), 6.92 \sim 6.90(\mathrm{~m}, 1 \mathrm{H}), 5.23(\mathrm{~d}, J=4.9 \mathrm{~Hz}, 1 \mathrm{H}), 3.91 \sim 3.88(\mathrm{~m}, 1 \mathrm{H})$, $3.72 \sim 3.69(\mathrm{~m}, 1 \mathrm{H}), 3.41 \sim 3.38(\mathrm{~m}, 1 \mathrm{H}), 2.45 \sim 2.43(\mathrm{~m}, 1 \mathrm{H}), 2.18 \sim 1.15(\mathrm{~m}, 1 \mathrm{H}), 1.41 \sim 1.34$ $(\mathrm{m}, 2 \mathrm{H}), 1.32(\mathrm{~d}, J=6.3 \mathrm{~Hz}, 3 \mathrm{H}), 1.20(\mathrm{~m}, 2 \mathrm{H}), 0.79(t, J=7.0 \mathrm{~Hz}, 3 \mathrm{H}) ;{ }^{13} \mathrm{C}$ NMR $(175 \mathrm{MHz}$, $\left.\mathrm{CDCl}_{3}\right): \delta 151.9,128.4,121.5,115.3,102.6,67.8,58.9,44.1,31.4,19.9,19.1,13.8$.

Spectral data of (3S,5S)-5-isobutoxy-3-methyl-2-phenylisoxazolidine (8c):

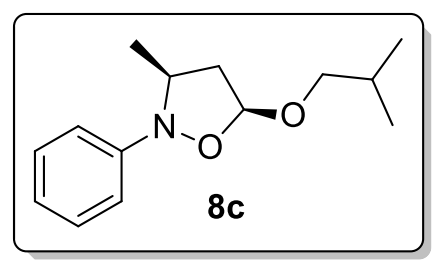

Compound $8 \mathbf{c}$ was purified on silica gel column using ethyl acetate/hexane: $(10: 90)$ as the eluent; Colorless liquid (73.7 mg, $0.31 \mathrm{mmol}, 67 \%) ;{ }^{1} \mathrm{H}$ NMR (700 MHz, $\left.\mathrm{CDCl}_{3}\right): \delta 7.28 \sim 7.25(\mathrm{~m}, 2 \mathrm{H})$, $7.10 \sim 7.07(\mathrm{~d}, J=7.7 \mathrm{~Hz} 2 \mathrm{H}), 7.02 \sim 7.00(\mathrm{~m}, 1 \mathrm{H}), 5.32(\mathrm{dd}, J=6.4,1.5 \mathrm{~Hz}, 1 \mathrm{H}), 3.65 \sim 3.63(\mathrm{~m}$, $1 \mathrm{H}), 3.52 \sim 3.50(\mathrm{~m}, 1 \mathrm{H}), 3.23 \sim 3.20(\mathrm{~m}, 1 \mathrm{H}), 2.56 \sim 2.52(\mathrm{~m}, 1 \mathrm{H}), 2.02 \sim 1.99(\mathrm{~m}, 1 \mathrm{H}), 1.93 \sim$ $1.89(\mathrm{~m}, 1 \mathrm{H}), 1.41(\mathrm{~d}, J=6.4 \mathrm{~Hz}, 3 \mathrm{H}), 0.92(t, J=7.1 \mathrm{~Hz}, 6 \mathrm{H}) ;{ }^{13} \mathrm{C} \mathrm{NMR}\left(175 \mathrm{MHz}, \mathrm{CDCl}_{3}\right): \delta$ 150.8, 128.6, 123.1, 117.3, 102.0, 75.4, 61.8, 42.5, 28.4, 19.7, 19.4, 19.3; HRMS (ESI-TOF) m/z: $[\mathrm{M}+\mathrm{H}]^{+}$calcd. For $\mathrm{C}_{14} \mathrm{H}_{21} \mathrm{NO}_{2}$ : 236.1650; found: 236.1656 .

Spectral data of (3S,5R)-5-isobutoxy-3-methyl-2-phenylisoxazolidine (8c'):

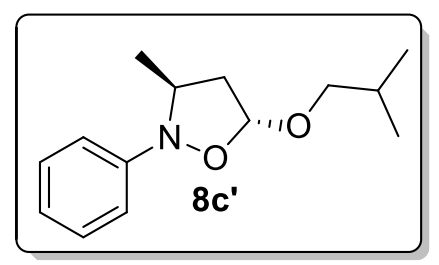

Compound $\mathbf{8} \mathbf{c}^{\prime}$ was purified on silica gel column using ethyl acetate/hexane: $(10: 90)$ as the eluent; Colorless liquid (16.5 mg, $0.07 \mathrm{mmol}, 15 \%) ;{ }^{1} \mathrm{H} \mathrm{NMR}\left(700 \mathrm{MHz}, \mathrm{CDCl}_{3}\right): \delta 7.25 \sim 7.22(\mathrm{~m}, 2 \mathrm{H})$, $7.04 \sim 7.07(\mathrm{~d}, J=7.9 \mathrm{~Hz} 2 \mathrm{H}), 6.93 \sim 6.91(\mathrm{~m}, 1 \mathrm{H}), 5.22(\mathrm{~d}, J=4.9 \mathrm{~Hz}, 1 \mathrm{H}), 3.93 \sim 3.89(\mathrm{~m}, 1 \mathrm{H})$, $3.50 \sim 3.47(\mathrm{~m}, 1 \mathrm{H}), 3.18 \sim 3.16(\mathrm{~m}, 1 \mathrm{H}), 2.48 \sim 2.45(\mathrm{~m}, 1 \mathrm{H}), 2.19 \sim 2.15(\mathrm{~m}, 1 \mathrm{H}), 1.69 \sim 1.65$ $(\mathrm{m}, 1 \mathrm{H}), 1.34(\mathrm{~d}, J=6.3 \mathrm{~Hz}, 3 \mathrm{H}), 0.77(\mathrm{~d}, J=6.6 \mathrm{~Hz}, 3 \mathrm{H}), 0.70(\mathrm{~d}, J=6.6 \mathrm{~Hz}, 3 \mathrm{H}) ;{ }^{13} \mathrm{C} \mathrm{NMR}$ (175 MHz, $\left.\mathrm{CDCl}_{3}\right): \delta$ 152.0, 128.4, 121.4, 115.4, 102.7, 74.7, 58.9, 44.2, 28.2, 20.0, 19.1, 19.0.

Spectral data of (3S,5S)-5-(cyclohexyloxy)-3-methyl-2-phenylisoxazolidine (8d): 


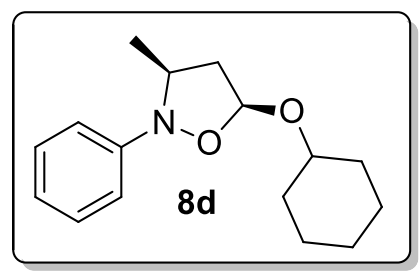

Compound 8d was purified on silica gel column using ethyl acetate/hexane: (7 :93) as the eluent; Colorless liquid (70.8 mg, $0.26 \mathrm{mmol}, 58 \%) ;{ }^{1} \mathrm{H}$ NMR (700 MHz, $\left.\mathrm{CDCl}_{3}\right): \delta 7.28 \sim 7.25$ (m, 2H), $7.10(\mathrm{~d}, J=7.5 \mathrm{~Hz}, 2 \mathrm{H}), 7.01 \sim 6.99(\mathrm{~m}, 1 \mathrm{H}), 5.50(\mathrm{dd}, J=6.3,2.1 \mathrm{~Hz}, 1 \mathrm{H}), 3.66 \sim 3.63(\mathrm{~m}, 1 \mathrm{H})$, $3.54 \sim 3.50(\mathrm{~m}, 1 \mathrm{H}), 2.51 \sim 2.49(\mathrm{~m}, 1 \mathrm{H}), 2.03 \sim 2.01(\mathrm{~m}, 1 \mathrm{H}), 1.98 \sim 1.95(\mathrm{~m}, 1 \mathrm{H}), 1.93 \sim 1.91$ $(\mathrm{m}, 1 \mathrm{H}), 1.75 \sim 1.71(\mathrm{~m}, 2 \mathrm{H}), 1.57 \sim 1.52(\mathrm{~m}, 1 \mathrm{H}), 1.41(\mathrm{~d}, J=6.4 \mathrm{~Hz}, 3 \mathrm{H}), 1.32 \sim 1.16(\mathrm{~m}, 5 \mathrm{H})$; ${ }^{13} \mathrm{C} \mathrm{NMR}\left(175 \mathrm{MHz}, \mathrm{CDCl}_{3}\right): \delta 150.9,128.6,122.9,117.1,100.1,76.4,61.8,42.7,33.8,31.9$, 25.7, 24.2, 24.1, 19.8; HRMS (ESI-TOF) m/z: $[\mathrm{M}+\mathrm{H}]^{+}$calcd. For $\mathrm{C}_{16} \mathrm{H}_{23} \mathrm{NO}_{2}$ : 262.1807; found: 262.1813.

\section{Spectral data of (3S,5R)-5-(cyclohexyloxy)-3-methyl-2-phenylisoxazolidine (8d'):}

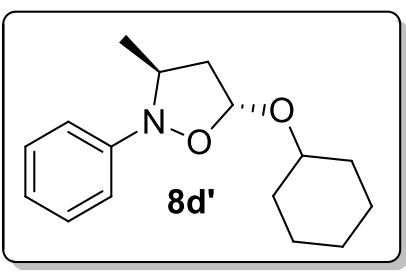

Compound 8d' was purified on silica gel column using ethyl acetate/hexane: (7:93) as the eluent; Colorless liquid (20.7 mg, 0.07mmol, 17\%); ${ }^{1} \mathrm{H}$ NMR (700 MHz, $\left.\mathrm{CDCl}_{3}\right): \delta 7.25 \sim 7.22(\mathrm{~m}, 2 \mathrm{H})$, $7.05(\mathrm{~d}, J=7.7 \mathrm{~Hz}, 2 \mathrm{H}), 6.93 \sim 6.91(\mathrm{~m}, 1 \mathrm{H}), 5.42(\mathrm{~d}, J=4.6 \mathrm{~Hz}, 1 \mathrm{H}), 3.94 \sim 3.89(\mathrm{~m}, 1 \mathrm{H}), 3.64$ $\sim 3.60(\mathrm{~m}, 1 \mathrm{H}), 2.43 \sim 2.40(\mathrm{~m}, 1 \mathrm{H}), 2.19 \sim 2.15(\mathrm{~m}, 1 \mathrm{H}), 1.92 \sim 1.90(\mathrm{~m}, 1 \mathrm{H}), 1.70 \sim 1.68(\mathrm{~m}$, $1 \mathrm{H}), 1.61 \sim 1.59(\mathrm{~m}, 1 \mathrm{H}), 1.50 \sim 1.48(\mathrm{~m}, 2 \mathrm{H}), 1.32(\mathrm{~d}, J=6.3 \mathrm{~Hz}, 3 \mathrm{H}), 1.24 \sim 1.07(\mathrm{~m}, 5 \mathrm{H}) ;{ }^{13} \mathrm{C}$ NMR $\left(175 \mathrm{MHz}, \mathrm{CDCl}_{3}\right): \delta 151.9,128.4,121.4,115.5,99.6,74.4,58.9,44.3,32.9,31.2,25.6$, 24.2, 24.0, 19.8 .

Spectral data of (3S,5S)-5-(tert-butoxy)-3-methyl-2-phenylisoxazolidine (8e): 


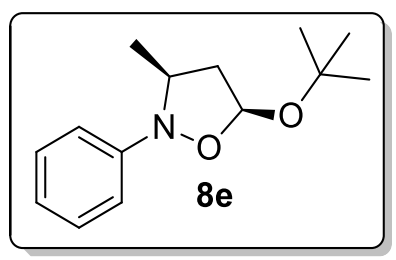

Compound 8e was purified on silica gel column using ethyl acetate/hexane: (7:93) as the eluent; yellow oil (82.2 mg, 0.35, 75\%); ${ }^{1} \mathrm{H}$ NMR (400 MHz, $\left.\mathrm{CDCl}_{3}\right): \delta 7.25 \sim 7.21(\mathrm{~m}, 2 \mathrm{H}), 7.10 \sim 7.07$ (m, 2H), $6.94 \sim 6.91(\mathrm{~m}, 1 \mathrm{H}), 5.32(\mathrm{dd}, J=5.3,2.7 \mathrm{~Hz}, 1 \mathrm{H}), 3.93 \sim 3.88(\mathrm{~m}, 1 \mathrm{H}), 2.35 \sim 3.30(\mathrm{~m}$, 1H), $3.21 \sim 3.15(\mathrm{~m}, 1 \mathrm{H}), 1.24(\mathrm{~s}, 9 \mathrm{H}), 1.22(\mathrm{~d}, J=6.3 \mathrm{~Hz}, 3 \mathrm{H}) ;{ }^{13} \mathrm{C} \mathrm{NMR}\left(100 \mathrm{MHz}, \mathrm{CDCl}_{3}\right): \delta$ 151.1, 128.3, 121.8, 116.9, 97.9, 74.7, 59.4, 44.3, 28.8, 18.6; HRMS (ESI-TOF) m/z: [M+H] ${ }^{+}$ calcd. For $\mathrm{C}_{14} \mathrm{H}_{21} \mathrm{NO}_{2}$ : 236.1650; found: 236.1648 .

Spectral data of (3S,5S)-5-ethoxy-3-methyl-2-(m-tolyl)isoxazolidine (8f):

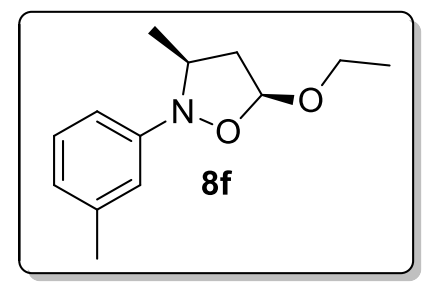

Compound $8 \mathbf{f}$ was purified on silica gel column using ethyl acetate/hexane: $(9: 91)$ as the eluent; Light yellow liquid (56.7 mg, $0.25 \mathrm{mmol}, 62 \%) ;{ }^{1} \mathrm{H} \mathrm{NMR}\left(700 \mathrm{MHz}, \mathrm{CDCl}_{3}\right): \delta 7.28 \sim 7.25(t, J$ $=7.7 \mathrm{~Hz}, 1 \mathrm{H}), 6.97(\mathrm{~s}, 1 \mathrm{H}), 6.93(\mathrm{~d}, J=8.0 \mathrm{~Hz}, 1 \mathrm{H}), 6.85(\mathrm{~d}, J=7.4 \mathrm{~Hz}, 1 \mathrm{H}), 5.31$ (dd, $J=6.3$, $1.6 \mathrm{~Hz}, 1 \mathrm{H}), 3.92 \sim 3.88(\mathrm{~m}, 1 \mathrm{H}), 3.55 \sim 3.51(\mathrm{~m}, 1 \mathrm{H}), 3.43 \sim 3.40(\mathrm{~m}, 1 \mathrm{H}), 2.60 \sim 2.56(\mathrm{~m}, 1 \mathrm{H})$, $2.31(\mathrm{~s}, 3 \mathrm{H}), 2.01 \sim 1.98(\mathrm{~m}, 1 \mathrm{H}), 1.37(\mathrm{~d}, J=6.0 \mathrm{~Hz}, 3 \mathrm{H}), 1.24(t, J=7.0 \mathrm{~Hz}, 3 \mathrm{H}) ;{ }^{13} \mathrm{C} \mathrm{NMR}(175$ $\left.\mathrm{MHz}, \mathrm{CDCl}_{3}\right): \delta 150.4,138.4,128.4,124.4,118.6,115.1,101.4,64.0,62.0,43.1,21.5,19.3,15.1$; HRMS (ESI-TOF) m/z: [M+H] ${ }^{+}$calcd. For $\mathrm{C}_{13} \mathrm{H}_{19} \mathrm{NO}_{2}$ : 222.1494; found: 222.1502 .

\section{Spectral data of (3S,5R)-5-ethoxy-3-methyl-2-(m-tolyl)isoxazolidine (8f'):}

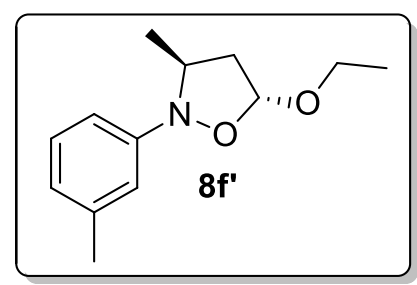


Compound 8f' was purified on silica gel column using ethyl acetate/hexane: $(9: 91)$ as the eluent; Light yellow liquid (15.5 mg, $0.07 \mathrm{mmol}, 17 \%) ;{ }^{1} \mathrm{H}$ NMR (700 MHz, $\left.\mathrm{CDCl}_{3}\right): \delta 7.11(t, J=7.7$ $\mathrm{Hz}, 1 \mathrm{H}), 6.85(\mathrm{~s}, 1 \mathrm{H}), 6.82(\mathrm{~d}, J=8.1 \mathrm{~Hz}, 1 \mathrm{H}), 6.73(\mathrm{~d}, J=7.3 \mathrm{~Hz}, 1 \mathrm{H}), 5.24(\mathrm{~d}, J=4.6 \mathrm{~Hz}, 1 \mathrm{H})$, $3.92 \sim 3.89(\mathrm{~m}, 1 \mathrm{H}), 3.80 \sim 3.78(\mathrm{~m}, 1 \mathrm{H}), 3.52 \sim 3.48(\mathrm{~m}, 1 \mathrm{H}), 2.45 \sim 2.42(\mathrm{~m}, 1 \mathrm{H}), 2.30(\mathrm{~s}, 3 \mathrm{H})$, $2.18 \sim 2.15(\mathrm{~m}, 1 \mathrm{H}), 1.30(\mathrm{~d}, J=6.3 \mathrm{~Hz}, 3 \mathrm{H}), 1.06(t, J=7.0 \mathrm{~Hz}, 3 \mathrm{H}) ;{ }^{13} \mathrm{C}$ NMR $(175 \mathrm{MHz}$, $\left.\mathrm{CDCl}_{3}\right): \delta 151.8,138.1,128.3,122.4,116.0,112.6,102.3,63.5,58.9,44.1,21.6,19.9,14.8$.

\section{Spectral data of (3S,5S)-5-ethoxy-2-(3-methoxyphenyl)-3-methylisoxazolidine (8g):}

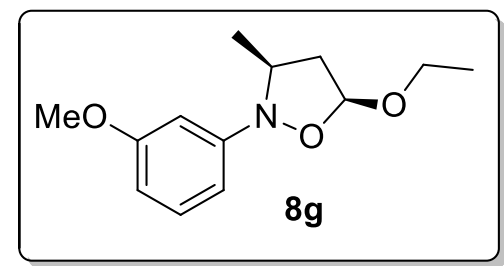

Compound $8 \mathbf{g}$ was purified on silica gel column using ethyl acetate/hexane: $(9: 91)$ as the eluent; Colorless liquid (53.7 mg, $0.22 \mathrm{mmol}, 62 \%) ;{ }^{1} \mathrm{H} \mathrm{NMR}\left(500 \mathrm{MHz}, \mathrm{CDCl}_{3}\right): \delta 7.16(t, J=8.0 \mathrm{~Hz}$, $1 \mathrm{H}), 6.73 \sim 6.69(\mathrm{~m}, 2 \mathrm{H}), 6.58 \sim 6.55(\mathrm{~m}, 1 \mathrm{H}), 5.32(\mathrm{dd}, J=6.25,2 \mathrm{~Hz}, 1 \mathrm{H}), 3.91 \sim 3.88(\mathrm{~m}, 1 \mathrm{H})$, $3.77(\mathrm{~s}, 3 \mathrm{H}), 3.55 \sim 3.52(\mathrm{~m}, 1 \mathrm{H}), 3.48 \sim 3.44(\mathrm{~m}, 1 \mathrm{H}), 2.60 \sim 2.54(\mathrm{~m}, 1 \mathrm{H}), 2.0 \sim 1.96(\mathrm{~m}, 1 \mathrm{H})$, $1.39(\mathrm{~d}, J=6.4 \mathrm{~Hz}, 3 \mathrm{H}), 1.24(t, J=7.1 \mathrm{~Hz}, 3 \mathrm{H}) ;{ }^{13} \mathrm{C} \mathrm{NMR}\left(125 \mathrm{MHz}, \mathrm{CDCl}_{3}\right): \delta 166.0,152.0$, 129.3, 110.0, 108.6, 103.7, 101.5, 64.1, 61.8, 55.2, 42.9, 19.6, 15.1; HRMS (ESI-TOF) m/z: [M+H] calcd. For $\mathrm{C}_{13} \mathrm{H}_{19} \mathrm{NO}_{3}$ : 238.1443; found: 238.1443 .

Spectral data of (3S,5R)-5-ethoxy-2-(3-methoxyphenyl)-3-methylisoxazolidine (8g'):

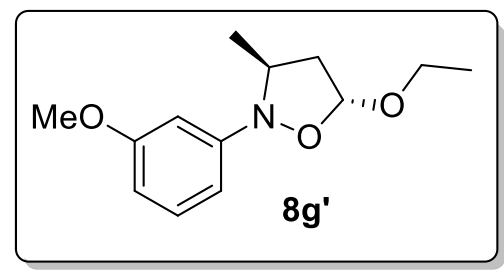

Compound $\mathbf{8 g}$ ' was purified on silica gel column using ethyl acetate/hexane: $(9: 91)$ as the eluent; Colorless liquid (14.7 mg, $0.06 \mathrm{mmol}, 17 \%)$; ${ }^{1} \mathrm{H} \mathrm{NMR}\left(400 \mathrm{MHz}, \mathrm{CDCl}_{3}\right): \delta 7.13(t, J=8.1 \mathrm{~Hz}$, 1H), $6.64 \sim 6.63(\mathrm{~m}, 1 \mathrm{H}), 6.59 \sim 6.57(\mathrm{~m}, 1 \mathrm{H}), 6.48 \sim 6.45(\mathrm{~m}, 1 \mathrm{H}), 5.25(\mathrm{~d}, J=4.4 \mathrm{~Hz}, 1 \mathrm{H}), 3.94$ $\sim 3.89(\mathrm{~m}, 1 \mathrm{H}), 3.81 \sim 3.77(\mathrm{~m}, 4 \mathrm{H}), 3.51 \sim 3.47(\mathrm{~m}, 1 \mathrm{H}), 2.47 \sim 2.42(\mathrm{~m}, 1 \mathrm{H}), 2.19 \sim 2.13(\mathrm{~m}$, $1 \mathrm{H}), 1.31(\mathrm{~d}, J=6.2 \mathrm{~Hz}, 3 \mathrm{H}), 1.05(t, J=7.0 \mathrm{~Hz}, 3 \mathrm{H}) ;{ }^{13} \mathrm{C} \mathrm{NMR}\left(100 \mathrm{MHz}, \mathrm{CDCl}_{3}\right): \delta 166.0$, 153.3, 129.2, 107.6, 106.7, 102.4, 101.5, 63.5, 58.8, 55.1, 42.1, 20.1, 14.8 . 
Spectral data of (3S,5S)-2-(3-chlorophenyl)-5-ethoxy-3-methylisoxazolidine (8h):

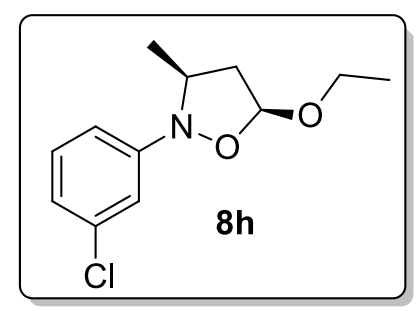

Compound $\mathbf{8 h}$ was purified on silica gel column using ethyl acetate/hexane: $(9: 91)$ as the eluent; Colorless liquid (41 mg, $0.16 \mathrm{mmol}, 48 \%) ;{ }^{1} \mathrm{H} \mathrm{NMR}\left(700 \mathrm{MHz}, \mathrm{CDCl}_{3}\right): \delta 7.17(t, J=8.0 \mathrm{~Hz}$, $1 \mathrm{H}), 7.13(\mathrm{~s}, 1 \mathrm{H}), 6.96 \sim 6.93(\mathrm{~m}, 2 \mathrm{H}), 5.33(\mathrm{dd}, J=5.0 \mathrm{~Hz}, 1 \mathrm{H}), 3.90 \sim 3.88(\mathrm{~m}, 1 \mathrm{H}), 3.56 \sim 3.53$ (m, 1H), $3.49 \sim 3.46(\mathrm{~m}, 1 \mathrm{H}), 2.57 \sim 2.53(\mathrm{~m}, 1 \mathrm{H}), 2.01 \sim 1.98(\mathrm{~m}, 1 \mathrm{H}), 1.40(\mathrm{~d}, J=6.4 \mathrm{~Hz}, 3 \mathrm{H})$, $1.24(t, J=7.0 \mathrm{~Hz}, 3 \mathrm{H}) ;{ }^{13} \mathrm{C} \mathrm{NMR}\left(175 \mathrm{MHz}, \mathrm{CDCl}_{3}\right): \delta 153.3,134.3,129.5,120.9,114.7,112.6$, 102.6, 63.7, 58.9, 43.9, 20.3, 14.7; HRMS (ESI-TOF) m/z: $[\mathrm{M}+\mathrm{H}]^{+}$calcd. For $\mathrm{C}_{12} \mathrm{H}_{16} \mathrm{ClNO}_{2}$ : 241.0870; found: 241.0869

Spectral data of (3S,5R)-2-(3-chlorophenyl)-5-ethoxy-3-methylisoxazolidine (8h'):

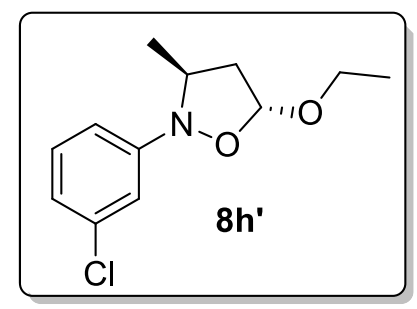

Compound 8h' was purified on silica gel column using ethyl acetate/hexane: $(9: 91)$ as the eluent; Colorless liquid (10.3 mg, $0.04 \mathrm{mmol}, 12 \%) ;{ }^{1} \mathrm{H} \mathrm{NMR}\left(700 \mathrm{MHz}, \mathrm{CDCl}_{3}\right): \delta 7.13(t, J=8.0 \mathrm{~Hz}$, 1H), $7.02(\mathrm{~s}, 1 \mathrm{H}), 6.86 \sim 6.83(\mathrm{~m}, 2 \mathrm{H}), 5.24(\mathrm{~d}, J=5.0 \mathrm{~Hz}, 1 \mathrm{H}), 3.93 \sim 3.90(\mathrm{~m}, 1 \mathrm{H}), 3.79 \sim 3.74$ $(\mathrm{m}, 1 \mathrm{H}), 3.52 \sim 3.47(\mathrm{~m}, 1 \mathrm{H}), 2.47 \sim 2.44(\mathrm{~m}, 1 \mathrm{H}), 2.17 \sim 2.13(\mathrm{~m}, 1 \mathrm{H}), 1.32(\mathrm{~d}, J=6.3 \mathrm{~Hz}, 3 \mathrm{H})$, $1.05(t, J=7.0 \mathrm{~Hz}, 3 \mathrm{H}) ;{ }^{13} \mathrm{C} \mathrm{NMR}\left(175 \mathrm{MHz}, \mathrm{CDCl}_{3}\right): \delta 151.9,134.4,129.6,122.9,117.1,115.3$, 101.6, 64.1, 61.8, 42.7, 19.8, 15.1.

Spectral data of (3S,5S)-2-(3-bromophenyl)-5-ethoxy-3-methylisoxazolidine (8i): 


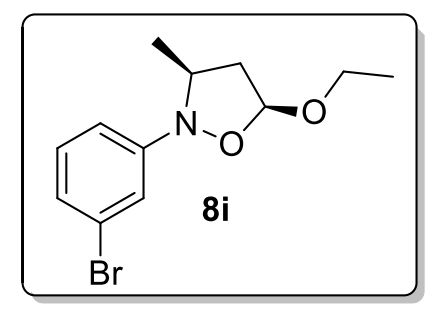

Compound 8i was purified on silica gel column using ethyl acetate/hexane: (10 : 90) as the eluent; yellow oil (53.8 mg, 0.18, 70\%); ${ }^{1} \mathrm{H}$ NMR (700 MHz, $\left.\mathrm{CDCl}_{3}\right)$ : $\delta 7.28$ (s, 1H), $7.11 \sim 7.10(\mathrm{~m}, 2 \mathrm{H})$, $6.99 \sim 6.97(\mathrm{~m}, 1 \mathrm{H}), 5.32(\mathrm{dd}, J=6.1,1.7 \mathrm{~Hz}, 1 \mathrm{H}), 3.90 \sim 3.87(\mathrm{~m}, 1 \mathrm{H}), 3.55 \sim 3.52(\mathrm{~m}, 1 \mathrm{H}), 3.48$ $\sim 3.45(\mathrm{~m}, 1 \mathrm{H}), 2.57 \sim 2.53(\mathrm{~m}, 1 \mathrm{H}), 2.01 \sim 1.98(\mathrm{~m}, 1 \mathrm{H}), 1.40(\mathrm{~d}, J=6.4 \mathrm{~Hz}, 3 \mathrm{H}), 1.24(t, J=7.0$ $\mathrm{Hz}, 3 \mathrm{H}) ;{ }^{13} \mathrm{C} \mathrm{NMR}\left(175 \mathrm{MHz}, \mathrm{CDCl}_{3}\right): \delta 152.1,129.9,125.8,122.6,119.9,115.8,101.5,64.7$, 61.8, 42.7, 19.8, 15.18; HRMS (ESI-TOF) m/z: $[\mathrm{M}+\mathrm{H}]^{+}$calcd. For $\mathrm{C}_{12} \mathrm{H}_{16} \mathrm{BrNO}_{2}$ : 286.0442; found: 286.0445 .

Spectral data of (3S,5R)-2-(3-bromophenyl)-5-ethoxy-3-methylisoxazolidine (8i'):

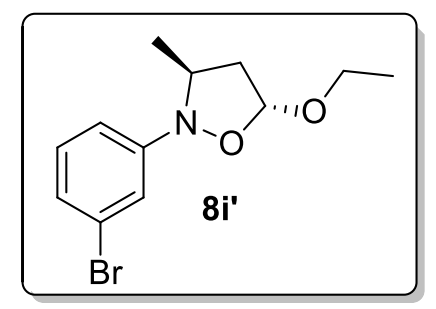

Compound 8i' was purified on silica gel column using ethyl acetate/hexane: (10:90) as the eluent; yellow oil (11.6 mg, $0.04 \mathrm{mmol}, 15 \%) ;{ }^{1} \mathrm{H}$ NMR (700 MHz, $\left.\mathrm{CDCl}_{3}\right): \delta 7.18(\mathrm{~s}, 1 \mathrm{H}), 7.07(t, J=$ $8.0 \mathrm{~Hz}, 1 \mathrm{H}) 7.01 \sim 7.00(\mathrm{~m}, 1 \mathrm{H}), 6.88 \sim 6.87(\mathrm{~m}, 1 \mathrm{H}), 5.24(\mathrm{~d}, J=5.0 \mathrm{~Hz}, 1 \mathrm{H}), 3.92 \sim 3.90(\mathrm{~m}$, $1 \mathrm{H}), 3.79 \sim 3.74(\mathrm{~m}, 1 \mathrm{H}), 3.52 \sim 3.48(\mathrm{~m}, 1 \mathrm{H}), 2.47 \sim 2.44(\mathrm{~m}, 1 \mathrm{H}), 2.17 \sim 2.13(\mathrm{~m}, 1 \mathrm{H}), 1.32(\mathrm{~d}$, $J=6.3 \mathrm{~Hz}, 3 \mathrm{H}), 1.05(t, J=7.0 \mathrm{~Hz}, 3 \mathrm{H}) ;{ }^{13} \mathrm{C} \mathrm{NMR}\left(175 \mathrm{MHz}, \mathrm{CDCl}_{3}\right): \delta 153.4,129.8,123.8$, $122.5,117.6,113.2,102.7,63.7,58.9,43.9,20.3,14.7$.

Spectral data of (3S,5S)-2-(4-chlorophenyl)-5-ethoxy-3-methylisoxazolidine (8j):

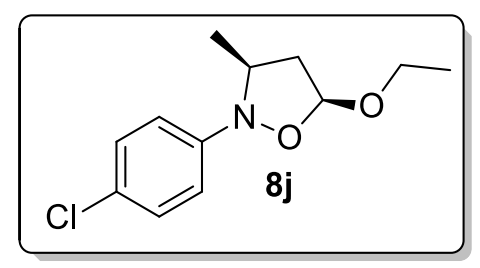


Compound $\mathbf{8 j}$ was purified on silica gel column using ethyl acetate/hexane: (10:90) as the eluent; Colorless oil (45.3 mg, $0.18 \mathrm{mmol}, 53 \%) ;{ }^{1} \mathrm{H} \mathrm{NMR}\left(700 \mathrm{MHz}, \mathrm{CDCl}_{3}\right): \delta 7.23(\mathrm{~d}, J=8.0 \mathrm{~Hz}, 2 \mathrm{H})$, $7.07(\mathrm{~d}, J=8.0 \mathrm{~Hz}, 2 \mathrm{H}), 5.30(\mathrm{dd}, J=6.1,2.1 \mathrm{~Hz}, 1 \mathrm{H}), 3.91 \sim 3.86(\mathrm{~m}, 1 \mathrm{H}), 3.55 \sim 3.51(\mathrm{~m}, 1 \mathrm{H})$, $3.39 \sim 3.36(\mathrm{~m}, 1 \mathrm{H}), 2.61 \sim 2.57(\mathrm{~m}, 1 \mathrm{H}), 2.02 \sim 1.99(\mathrm{~m}, 1 \mathrm{H}), 1.37(\mathrm{~d}, J=6.4 \mathrm{~Hz}, 3 \mathrm{H}), 1.24(t, J$ $=7.0 \mathrm{~Hz}, 3 \mathrm{H}) ;{ }^{13} \mathrm{C} \mathrm{NMR}\left(175 \mathrm{MHz}, \mathrm{CDCl}_{3}\right): \delta 149.0,128.6,128.5,119.1,101.4,64.1,62.2,43.1$, 19.3, 15.1; HRMS (ESI-TOF) m/z: [M+H] ${ }^{+}$calcd. For $\mathrm{C}_{12} \mathrm{H}_{16} \mathrm{ClNO}_{2}$ : 241.0870; found: 241.0873.

\section{Spectral data of (3S,5S)-2-(4-bromophenyl)-5-ethoxy-3-methylisoxazolidine (8k):}

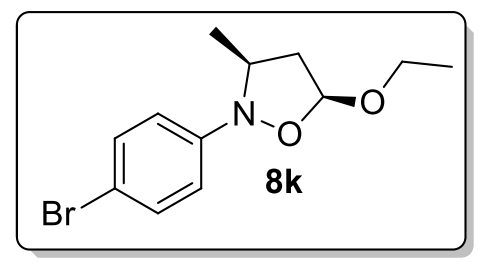

Compound 8k was purified on silica gel column using ethyl acetate/hexane: $(10: 90)$ as the eluent; Colorless oil (39.3 mg, $0.13 \mathrm{mmol}, 51 \%) ;{ }^{1} \mathrm{H}$ NMR (700 MHz, $\left.\mathrm{CDCl}_{3}\right): \delta 7.36(\mathrm{~d}, J=8.0 \mathrm{~Hz}, 2 \mathrm{H})$, $7.01(\mathrm{~d}, J=8.0 \mathrm{~Hz}, 2 \mathrm{H}), 5.30(\mathrm{dd}, J=6.1,1.9 \mathrm{~Hz}, 1 \mathrm{H}), 3.90 \sim 3.86(\mathrm{~m}, 1 \mathrm{H}), 3.55 \sim 3.50(\mathrm{~m}, 1 \mathrm{H})$, $3.40 \sim 3.37(\mathrm{~m}, 1 \mathrm{H}), 2.59 \sim 2.55(\mathrm{~m}, 1 \mathrm{H}), 2.01 \sim 1.98(\mathrm{~m}, 1 \mathrm{H}), 1.37(\mathrm{~d}, J=6.4 \mathrm{~Hz}, 3 \mathrm{H}), 1.23(t, J$ $=7.0 \mathrm{~Hz}, 3 \mathrm{H}) ;{ }^{13} \mathrm{C} \mathrm{NMR}\left(175 \mathrm{MHz}, \mathrm{CDCl}_{3}\right): \delta 149.6,131.5,119.3,115.9,101.4,64.1,62.0,43.0$, 19.4, 15.1; HRMS (ESI-TOF) m/z: [M+H] ${ }^{+}$calcd. For $\mathrm{C}_{12} \mathrm{H}_{16} \mathrm{BrNO}_{2}$ : 286.0442; found: 286.0436. Spectral data of (3S,5S)-5-ethoxy-2-(4-fluorophenyl)-3-methylisoxazolidine (81):

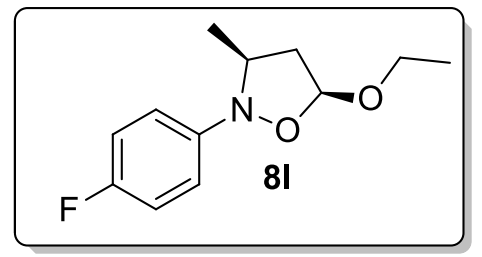

Compound $\mathbf{8 1}$ was purified on silica gel column using ethyl acetate/hexane: $(10: 90)$ as the eluent; Colorless oil (55 mg, $0.24 \mathrm{mmol}, 61 \%) ;{ }^{1} \mathrm{H}$ NMR (700 MHz, $\left.\mathrm{CDCl}_{3}\right): \delta 7.17 \sim 7.15$ (m, 2H), 6.99 $\sim 6.96(\mathrm{~m}, 2 \mathrm{H}), 5.28(\mathrm{dd}, J=6.3,2.4 \mathrm{~Hz}, 1 \mathrm{H}), 3.89 \sim 3.86(\mathrm{~m}, 1 \mathrm{H}), 3.53 \sim 3.50(\mathrm{~m}, 1 \mathrm{H}), 3.25 \sim$ $3.23(\mathrm{~m}, 1 \mathrm{H}), 2.66 \sim 2.62(\mathrm{~m}, 1 \mathrm{H}), 2.03 \sim 2.0(\mathrm{~m}, 1 \mathrm{H}), 1.30(\mathrm{~d}, J=6.3 \mathrm{~Hz}, 3 \mathrm{H}), 1.23(t, J=7.0$ $\mathrm{Hz}, 3 \mathrm{H}) ;{ }^{13} \mathrm{C}$ NMR $\left(175 \mathrm{MHz}, \mathrm{CDCl}_{3}\right): \delta 160.5,120.8,115.3,115.2,101.1,64.1,62.8,43.7,18.3$, 15.1; HRMS (ESI-TOF) m/z: [M+H] ${ }^{+}$calcd. For $\mathrm{C}_{12} \mathrm{H}_{16} \mathrm{FNO}_{2}$ : 226.1243; found: 226.1243 .

Spectral data of (3S,5S)-5-ethoxy-3-methyl-2-(4-(trifluoromethyl)phenyl)isoxazolidine (8n): 


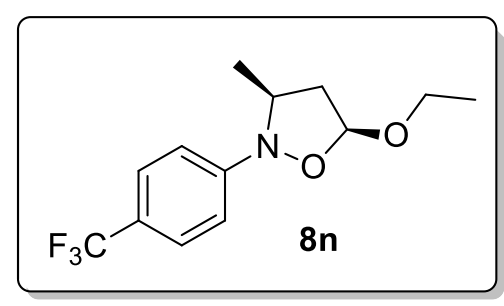

Compound 8n was purified on silica gel column using ethyl acetate/hexane: (10:90) as the eluent; Colorless liquid (51.1 mg, $\left.0.18 \mathrm{mmol}, 65 \%) ;{ }^{1} \mathrm{H} \mathrm{NMR} \mathrm{(700} \mathrm{MHz,} \mathrm{CDCl}_{3}\right): \delta 7.49(\mathrm{~d}, J=8.5 \mathrm{~Hz}$, 2H), $7.10(\mathrm{~d}, J=8.5 \mathrm{~Hz}, 2 \mathrm{H}), 5.34(\mathrm{dd}, J=6.0,1.5 \mathrm{~Hz}, 1 \mathrm{H}), 3.92 \sim 3.88(\mathrm{~m}, 1 \mathrm{H}), 3.64 \sim 3.59(\mathrm{~m}$, $1 \mathrm{H}), 3.58 \sim 3.53(\mathrm{~m}, 1 \mathrm{H}), 2.56 \sim 3.52(\mathrm{~m}, 1 \mathrm{H}), 2.03 \sim 2.01(\mathrm{~m}, 1 \mathrm{H}), 1.47(\mathrm{~d}, J=6.4 \mathrm{~Hz}, 3 \mathrm{H}), 1.25$ $(t, J=7.0 \mathrm{~Hz}, 3 \mathrm{H}) ;{ }^{13} \mathrm{C} \mathrm{NMR}\left(175 \mathrm{MHz}, \mathrm{CDCl}_{3}\right): \delta 153.3,125.8,125.7,123.7,115.4,101.5,64.1$, 60.8, 42.3, 20.3, 15.0; HRMS (EI-MS) m/z: $[\mathrm{M}]^{+}$calcd. For $\mathrm{C}_{13} \mathrm{H}_{16} \mathrm{~F}_{3} \mathrm{NO}_{2}$ : 275.1133; found: 275.1130 .

Spectral data of (3S,5R)-5-ethoxy-3-methyl-2-(4-(trifluoromethyl)phenyl)isoxazolidine (8n'):

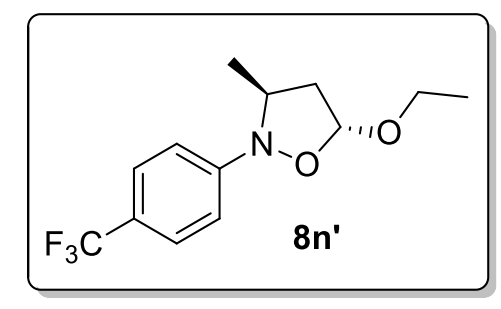

Compound 8n' was purified on silica gel column using ethyl acetate/hexane: (10:90) as the eluent; Colorless liquid (15.7 mg, $0.05 \mathrm{mmol}, 20 \%) ;{ }^{1} \mathrm{H} \mathrm{NMR}\left(700 \mathrm{MHz}, \mathrm{CDCl}_{3}\right): \delta 7.46(\mathrm{~d}, J=8.5 \mathrm{~Hz}$, 2H), $7.01(\mathrm{~d}, J=8.5 \mathrm{~Hz}, 2 \mathrm{H}), 5.26(\mathrm{~d}, J=4.9 \mathrm{~Hz}, 1 \mathrm{H}), 4.01 \sim 3.98(\mathrm{~m}, 1 \mathrm{H}), 3.76 \sim 3.71(\mathrm{~m}, 1 \mathrm{H})$, $3.52 \sim 3.47(\mathrm{~m}, 1 \mathrm{H}), 2.50 \sim 2.47(\mathrm{~m}, 1 \mathrm{H}), 2.19 \sim 2.15(\mathrm{~m}, 1 \mathrm{H}), 1.36(\mathrm{~d}, J=6.3 \mathrm{~Hz}, 3 \mathrm{H}), 1.01(t, J$ $=7.0 \mathrm{~Hz}, 3 \mathrm{H}) ;{ }^{13} \mathrm{C} \mathrm{NMR}\left(175 \mathrm{MHz}, \mathrm{CDCl}_{3}\right): \delta 154.5,125.84,125.82,125.80,113.3,102.6,63.7$, 58.3, 44.0, 20.5, 14.7 .

Spectral data of ethyl 4-((3S,5S)-5-ethoxy-3-methylisoxazolidin-2-yl)benzoate (8o): 


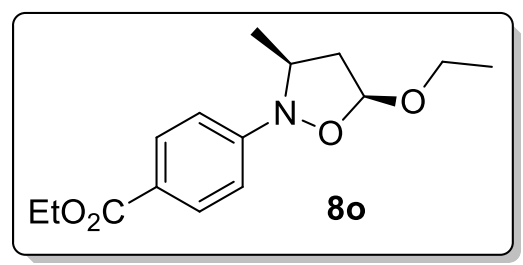

Compound 80 was purified on silica gel column using ethyl acetate/hexane: (10:90) as the eluent; Colorless oil (44.4 mg, $0.15 \mathrm{mmol}, 57 \%) ;{ }^{1} \mathrm{H} \mathrm{NMR}\left(700 \mathrm{MHz}, \mathrm{CDCl}_{3}\right): \delta 7.93$ (d, J=8.5 Hz, 2H), $7.02(\mathrm{~d}, J=8.5 \mathrm{~Hz}, 2 \mathrm{H}), 5.34(\mathrm{dd}, J=5.8,1.1 \mathrm{~Hz}, 1 \mathrm{H}), 4.32 \sim 4.30(\mathrm{~m}, 2 \mathrm{H}), 3.92 \sim 3.89(\mathrm{~m}, 1 \mathrm{H})$, $3.71 \sim 3.68(\mathrm{~m}, 1 \mathrm{H}), 3.58 \sim 3.55(\mathrm{~m}, 1 \mathrm{H}), 2.55 \sim 2.51(\mathrm{~m}, 1 \mathrm{H}), 2.03 \sim 2.0(\mathrm{~m}, 1 \mathrm{H}), 1.48(\mathrm{~d}, J=6.4$ $\mathrm{Hz}, 3 \mathrm{H}), 1.35(t, J=7.0 \mathrm{~Hz}, 3 \mathrm{H}), 1.35(t, J=7.0 \mathrm{~Hz}, 3 \mathrm{H}) ;{ }^{13} \mathrm{C} \mathrm{NMR}\left(175 \mathrm{MHz}, \mathrm{CDCl}_{3}\right): \delta 166.5$, 154.3, 130.5, 123.4, 114.4, 101.6, 64.1, 60.5, 60.3 42.3, 20.7, 15.2, 14.3; HRMS (ESI-TOF) m/z: $[\mathrm{M}+\mathrm{H}]$ calcd. For $\mathrm{C}_{15} \mathrm{H}_{21} \mathrm{NO}_{4}$ : 280.1548; found: 280.1544 .

Spectral data of ethyl 4-((3S,5R)-5-ethoxy-3-methylisoxazolidin-2-yl)benzoate (80'):

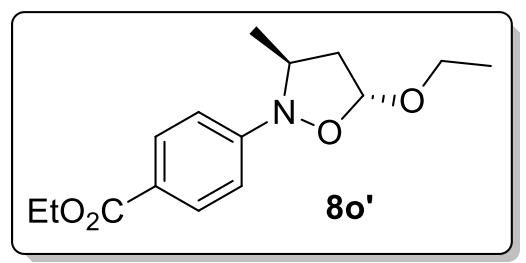

Compound 80' was purified on silica gel column using ethyl acetate/hexane: (10:90) as the eluent; Colorless liquid (12.5 mg, $\left.0.04 \mathrm{mmol}, 16 \%) ;{ }^{1} \mathrm{H} \mathrm{NMR} \mathrm{(700} \mathrm{MHz,} \mathrm{CDCl}_{3}\right): \delta 7.91(\mathrm{~d}, J=8.5 \mathrm{~Hz}$, 2H), $6.95(\mathrm{~d}, J=8.5 \mathrm{~Hz}, 2 \mathrm{H}), 5.25(\mathrm{~d}, J=4.9,1 \mathrm{H}), 4.32 \sim 4.29(\mathrm{~m}, 2 \mathrm{H}), 4.03 \sim 4.01(\mathrm{~m}, 1 \mathrm{H}), 3.75$ $\sim 3.73(\mathrm{~m}, 1 \mathrm{H}), 3.49 \sim 3.47(\mathrm{~m}, 1 \mathrm{H}), 2.50 \sim 2.47(\mathrm{~m}, 1 \mathrm{H}), 2.18 \sim 2.14(\mathrm{~m}, 1 \mathrm{H}), 1.35(\mathrm{~m}, 6 \mathrm{H}), 1.0$ $(t, J=7.0 \mathrm{~Hz}, 3 \mathrm{H}) ;{ }^{13} \mathrm{C} \mathrm{NMR}\left(175 \mathrm{MHz}, \mathrm{CDCl}_{3}\right): \delta 166.6,154.4,130.5,122.1,112.6,102.5,63.7$, $60.4,57.9,44.0,20.6,14.7,14.3$.

\section{Computational Details:}

Geometries were optimized using the B3LYP functional, the $6-31 \mathrm{G}^{* *}$ basis for light atoms, and a double- $\zeta$ contraction of the Los Alamos valence functions and the corresponding pseudopotential for gold. Hessians at these geometries provided the zero-point energy (ZPE) and vibrational enthalpy $\left(\mathrm{H}_{\mathrm{vib}}\right)$. Solvation energies $\mathrm{G}_{\text {solv }}$ were computed in dichloromethane with the CPCM continuum model. The electronic energy $\mathrm{E}_{\text {elec }}$ was evaluated with the same functional and the 6- 
$311++\mathrm{G}^{* *}$ basis and a triple $-\zeta$ contraction of the Los Alamos valence functions augmented with diffuse $s$ and $p$ functions for gold. Enthalpy of all species were computed according to the equation as follows:

$\mathrm{H}=\mathrm{E}_{\text {elec }}+\mathrm{G}_{\mathrm{solv}}+\mathrm{ZPE}+\mathrm{H}_{\mathrm{vib}}+4 \mathrm{kT}$

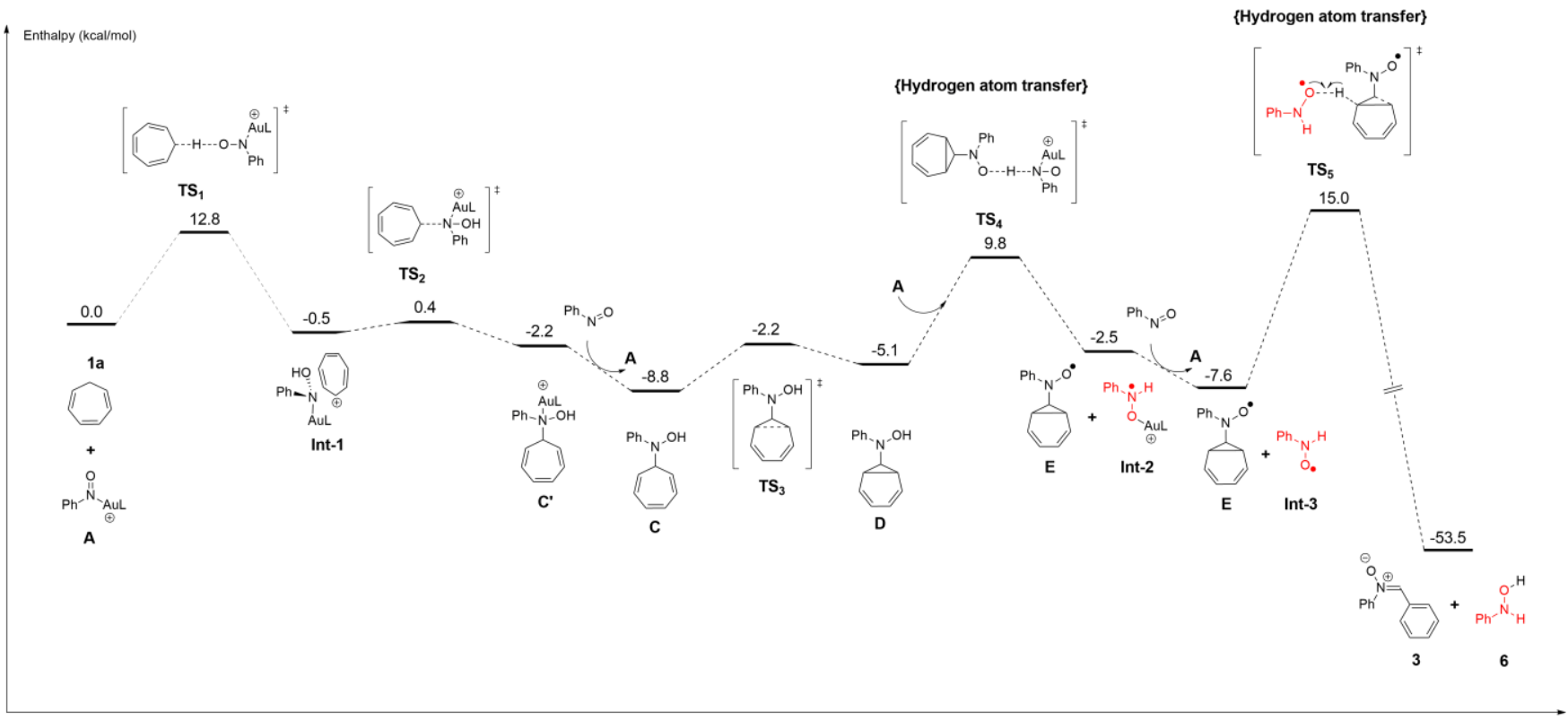

Figure S1. Energy Profile for Transitions states and Intermediates

\begin{tabular}{|c|c|c|c|c|}
\hline Species & $\mathrm{E}_{\text {elec }}$ & $\mathrm{G}_{\text {solv }}$ & $\mathrm{ZPE}+\mathrm{H}_{\text {vib }}+4 \mathrm{kT}$ & $\mathrm{H}_{\text {total }}($ hartree $)$ \\
\hline $\mathbf{1 a}$ & -271.58459 & -0.00218 & 0.13480 & -271.45198 \\
\hline $\mathbf{A}$ & -1616.95004 & -0.04800 & 0.55243 & -1616.44561 \\
\hline int1 & -1888.54025 & -0.04825 & 0.69010 & -1887.89840 \\
\hline $\mathbf{C}^{\prime}$ & -1888.54485 & -0.04745 & 0.69120 & -1887.90110 \\
\hline $\mathbf{C}$ & -633.24140 & -0.00647 & 0.24250 & -633.00537 \\
\hline nitrosobenzene & -361.63917 & -0.00483 & 0.10455 & -361.53945 \\
\hline $\mathbf{D}$ & -633.23531 & -0.00712 & 0.24295 & -632.99948 \\
\hline $\mathbf{E}$ & -632.62125 & -0.00593 & 0.23104 & -632.39614 \\
\hline int2 & -1617.55901 & -0.04992 & 0.56399 & -1617.04494 \\
\hline int3 & -362.25630 & -0.00706 & 0.11656 & -362.14680 \\
\hline $\mathbf{3}$ & -632.08274 & -0.00785 & 0.22082 & -631.86977 \\
\hline $\mathbf{6}$ & -362.87335 & -0.00645 & 0.12934 & -362.75046 \\
\hline TS & -1888.51455 & -0.04668 & 0.68398 & -1887.87725 \\
\hline
\end{tabular}




\begin{tabular}{|l|l|l|l|l|}
\hline $\mathbf{T S}_{\mathbf{2}}$ & -1888.53863 & -0.04764 & 0.68937 & -1887.89691 \\
\hline $\mathbf{T S}_{\mathbf{3}}$ & -633.22896 & -0.00730 & 0.24140 & -632.99486 \\
\hline $\mathbf{T S}_{\mathbf{4}}$ & -2250.16923 & -0.04499 & 0.79279 & -2249.42143 \\
\hline $\mathbf{T S}_{\mathbf{5}}$ & -994.84387 & -0.01020 & 0.34704 & -994.50703 \\
\hline
\end{tabular}

\section{Coordinates of optimized structures}

$\begin{array}{rrrr}\text { 1a } & & & \\ \mathrm{C} & -1.43092800 & 0.68263600 & 0.18479700 \\ \mathrm{C} & -1.43092200 & -0.68265000 & 0.18479700 \\ \mathrm{C} & -0.34859900 & -1.53113500 & -0.26404000 \\ \mathrm{C} & -0.34861400 & 1.53113200 & -0.26404000 \\ \mathrm{C} & 0.96518000 & -1.22271600 & -0.20244100 \\ \mathrm{C} & 0.96516800 & 1.22272500 & -0.20244100 \\ \mathrm{C} & 1.46347500 & 0.00000700 & 0.52790300 \\ \mathrm{H} & -2.37043900 & 1.17965500 & 0.42103000 \\ \mathrm{H} & -2.37042700 & -1.17967700 & 0.42103100 \\ \mathrm{H} & -0.63678700 & -2.48319900 & -0.70783300 \\ \mathrm{H} & -0.63681000 & 2.48319300 & -0.70783300 \\ \mathrm{H} & 1.69189900 & -1.87607100 & -0.68045600 \\ \mathrm{H} & 1.69188100 & 1.87608600 & -0.68045600 \\ \mathrm{H} & 1.06762600 & 0.00000500 & 1.55383700 \\ \mathrm{H} & 2.55449900 & 0.00001200 & 0.59347000\end{array}$

A

$\begin{array}{crrr}\mathrm{Au} & 0.60137600 & -0.24865200 & -0.35824900 \\ \mathrm{P} & -1.63037600 & -0.89156400 & -0.00476700 \\ \mathrm{C} & -1.68329300 & -1.99950700 & 1.55655600 \\ \mathrm{C} & -2.19992200 & -1.74543200 & -1.63357700 \\ \mathrm{C} & -2.76142600 & 0.53733800 & 0.30302100 \\ \mathrm{C} & -2.33322100 & 1.88598400 & 0.39734200 \\ \mathrm{C} & -4.14020500 & 0.26027700 & 0.41616100 \\ \mathrm{C} & -3.30287200 & 2.88520200 & 0.59637900 \\ \mathrm{C} & -0.92135900 & 2.38365900 & 0.30344200 \\ \mathrm{C} & -5.08090300 & 1.26622100 & 0.61430000 \\ \mathrm{H} & -4.49369400 & -0.75959600 & 0.34922000 \\ \mathrm{C} & -4.65854000 & 2.59090100 & 0.70445400 \\ \mathrm{H} & -2.96963400 & 3.91572600 & 0.66829000 \\ \mathrm{C} & -0.11140700 & 2.46664900 & 1.44891600 \\ \mathrm{C} & -0.44977100 & 2.93728900 & -0.89882100 \\ \mathrm{H} & -6.13308600 & 1.01256900 & 0.69543300 \\ \mathrm{H} & -5.37679800 & 3.39024700 & 0.85788500 \\ \mathrm{C} & 1.14208900 & 3.08189200 & 1.38961400 \\ \mathrm{H} & -0.47843600 & 2.07688300 & 2.39360100 \\ \mathrm{C} & 0.80499900 & 3.54792400 & -0.95715200\end{array}$




$\begin{array}{lrrr}\mathrm{H} & -1.07894400 & 2.90650200 & -1.78357200 \\ \mathrm{C} & 1.60147500 & 3.62580400 & 0.18775400 \\ \mathrm{H} & 1.74627200 & 3.15788100 & 2.28899600 \\ \mathrm{H} & 1.15059400 & 3.97747300 & -1.89229600 \\ \mathrm{H} & 2.56633600 & 4.12227500 & 0.14735300 \\ \mathrm{C} & -0.84731400 & -3.26927100 & 1.29814100 \\ \mathrm{H} & 0.15305000 & -3.03791700 & 0.91843000 \\ \mathrm{H} & -0.72920500 & -3.81146700 & 2.24276300 \\ \mathrm{H} & -1.33467600 & -3.94906800 & 0.59461000 \\ \mathrm{C} & -1.03074100 & -1.17480400 & 2.68798600 \\ \mathrm{H} & 0.00616600 & -0.90863400 & 2.46169200 \\ \mathrm{H} & -1.58530900 & -0.25443200 & 2.89258300 \\ \mathrm{H} & -1.03261500 & -1.77308700 & 3.60582400 \\ \mathrm{C} & -3.09947900 & -2.40270100 & 2.01391200 \\ \mathrm{H} & -3.65434300 & -2.95703200 & 1.25467700 \\ \mathrm{H} & -3.00125700 & -3.05836400 & 2.88623100 \\ \mathrm{H} & -3.69176600 & -1.53965200 & 2.32364800 \\ \mathrm{C} & -1.04951900 & -2.62927900 & -2.16663000 \\ \mathrm{H} & -0.15079800 & -2.04501400 & -2.38340400 \\ \mathrm{H} & -0.77713600 & -3.43386400 & -1.48078800 \\ \mathrm{H} & -1.37691500 & -3.09256000 & -3.10386000 \\ \mathrm{C} & -3.46140800 & -2.62065100 & -1.49941600 \\ \mathrm{H} & -4.33766100 & -2.06272200 & -1.16505800 \\ \mathrm{H} & -3.70273200 & -3.02556200 & -2.48843200 \\ \mathrm{H} & -3.30864300 & -3.47310400 & -0.83301900 \\ \mathrm{C} & -2.45719900 & -0.61090700 & -2.64873600 \\ \mathrm{H} & -3.31594500 & 0.00556900 & -2.37416700 \\ \mathrm{H} & -1.58448400 & 0.04129100 & -2.76144500 \\ \mathrm{H} & -2.66221300 & -1.05806100 & -3.62747200 \\ \mathrm{C} & 3.80452200 & -0.19422000 & -0.18889700 \\ \mathrm{C} & 5.08757200 & 0.17186900 & -0.64145800 \\ \mathrm{C} & 3.63095200 & -0.99097200 & 0.95467500 \\ \mathrm{C} & 6.19466600 & -0.26895000 & 0.06573500 \\ \mathrm{H} & 5.18088400 & 0.78590900 & -1.52947600 \\ \mathrm{C} & 4.75069700 & -1.42485000 & 1.65465000 \\ \mathrm{H} & 6.62848600 & -1.25781000 & 1.27571600 \\ \mathrm{H} & 7.19202700 & -0.00006200 & -0.26590600 \\ \mathrm{H} & 4.63489800 & -2.04068200 & 2.54000700 \\ \mathrm{H} & 2.79012400 & -1.40585500 & 1.75723900 \\ \mathrm{H} & 0.93398500 & -1.86828800 \\ \mathrm{H} & 0.23272400 & -0.87988200\end{array}$

int1

$\mathrm{Au} \quad 0.01510400 \quad-0.16904400 \quad-0.24273100$

$\begin{array}{llll}\mathrm{P} & 2.19743900 & -0.98022200 & 0.10408100\end{array}$ 


\begin{tabular}{|c|c|c|c|}
\hline $\mathrm{C}$ & 2.59229300 & -2.06916100 & -1.42636000 \\
\hline $\mathrm{C}$ & 2.19346000 & -1.92519900 & 1.78389500 \\
\hline $\mathrm{C}$ & 3.54738400 & 0.28459000 & 0.21659700 \\
\hline $\mathrm{C}$ & 3.35031400 & 1.68310900 & 0.10010200 \\
\hline $\mathrm{C}$ & 4.85443500 & -0.18302600 & 0.46924200 \\
\hline $\mathrm{C}$ & 4.46014500 & 2.53661100 & 0.24020100 \\
\hline $\mathrm{C}$ & 2.05468900 & 2.39247000 & -0.16202700 \\
\hline $\mathrm{C}$ & 5.93877600 & 0.67860100 & 0.60413000 \\
\hline $\mathrm{H}$ & 5.03735500 & -1.24419400 & 0.56234300 \\
\hline $\mathrm{C}$ & 5.74041700 & 2.05240600 & 0.48834900 \\
\hline $\mathrm{H}$ & 4.30016900 & 3.60626100 & 0.14718700 \\
\hline $\mathrm{C}$ & 1.63181400 & 2.64883800 & -1.47495300 \\
\hline $\mathrm{C}$ & 1.34930700 & 2.97915000 & 0.90175000 \\
\hline $\mathrm{H}$ & 6.92755000 & 0.27497300 & 0.79799400 \\
\hline $\mathrm{H}$ & 6.57226500 & 2.74247800 & 0.58948900 \\
\hline $\mathrm{C}$ & 0.53209400 & 3.47669900 & -1.72085500 \\
\hline $\mathrm{H}$ & 2.18462600 & 2.22671400 & -2.30841800 \\
\hline $\mathrm{C}$ & 0.24787100 & 3.80211100 & 0.65916700 \\
\hline $\mathrm{H}$ & 1.68566900 & 2.81073800 & 1.92057700 \\
\hline $\mathrm{C}$ & -0.16065200 & 4.05963000 & -0.65412200 \\
\hline $\mathrm{H}$ & 0.23541700 & 3.68692200 & -2.74424900 \\
\hline $\mathrm{H}$ & -0.26802300 & 4.27135300 & 1.49214600 \\
\hline $\mathrm{H}$ & -0.99171900 & 4.73218500 & -0.84671600 \\
\hline $\mathrm{C}$ & 1.57939100 & -3.23162300 & -1.48172000 \\
\hline $\mathrm{H}$ & 0.54433100 & -2.88478800 & -1.40332000 \\
\hline $\mathrm{H}$ & 1.68475900 & -3.74298700 & -2.44488900 \\
\hline $\mathrm{H}$ & 1.76005200 & -3.97502400 & -0.70053300 \\
\hline $\mathrm{C}$ & 2.38629500 & -1.15682300 & -2.65681700 \\
\hline $\mathrm{H}$ & 1.35803000 & -0.79100800 & -2.73272300 \\
\hline $\mathrm{H}$ & 3.06322800 & -0.29705700 & -2.64397800 \\
\hline $\mathrm{H}$ & 2.60410600 & -1.73420100 & -3.56220600 \\
\hline $\mathrm{C}$ & 4.02377100 & -2.63719300 & -1.48599500 \\
\hline $\mathrm{H}$ & 4.27716400 & -3.25895300 & -0.62496800 \\
\hline $\mathrm{H}$ & 4.10001900 & -3.27185500 & -2.37595400 \\
\hline $\mathrm{H}$ & 4.77454400 & -1.85107500 & -1.58650200 \\
\hline $\mathrm{C}$ & 0.84631700 & -2.66746600 & 1.93301200 \\
\hline $\mathrm{H}$ & 0.00069200 & -1.97430100 & 1.92205300 \\
\hline $\mathrm{H}$ & 0.68407000 & -3.41550500 & 1.15436600 \\
\hline $\mathrm{H}$ & 0.84102400 & -3.18795900 & 2.89748300 \\
\hline $\mathrm{C}$ & 3.32897400 & -2.94993500 & 1.97156200 \\
\hline $\mathrm{H}$ & 4.32283100 & -2.50123400 & 1.93283800 \\
\hline $\mathrm{H}$ & 3.22462100 & -3.40063100 & 2.96505500 \\
\hline $\mathrm{H}$ & 3.27966500 & -3.76339600 & 1.24383000 \\
\hline $\mathrm{C}$ & 2.27913600 & -0.84886500 & 2.88756000 \\
\hline $\mathrm{H}$ & 3.24611200 & -0.34104500 & 2.89797000 \\
\hline $\mathrm{H}$ & 1.49386100 & -0.09272700 & 2.77855200 \\
\hline
\end{tabular}




\begin{tabular}{|c|c|c|c|}
\hline $\mathrm{H}$ & 2.14231900 & -1.33132700 & 3.86182100 \\
\hline $\mathrm{C}$ & -2.95537000 & -0.55324400 & -0.92838400 \\
\hline $\mathrm{C}$ & -3.99675500 & -0.31837900 & -1.85770000 \\
\hline $\mathrm{C}$ & -2.94870600 & -1.77573300 & -0.21092100 \\
\hline $\mathrm{C}$ & -4.98886800 & -1.27776200 & -2.04621800 \\
\hline $\mathrm{H}$ & -3.99612600 & 0.59462500 & -2.43681300 \\
\hline $\mathrm{C}$ & -3.95183300 & -2.71520500 & -0.41093000 \\
\hline $\mathrm{H}$ & -2.13872300 & -1.97937400 & 0.48244700 \\
\hline $\mathrm{C}$ & -4.98801000 & -2.47065300 & -1.31991600 \\
\hline $\mathrm{H}$ & -5.77193600 & -1.09003600 & -2.77566400 \\
\hline $\mathrm{H}$ & -3.92409200 & -3.64983500 & 0.14232800 \\
\hline $\mathrm{H}$ & -5.76406700 & -3.21242500 & -1.47934100 \\
\hline $\mathrm{O}$ & -2.10918900 & 1.49593500 & -1.53027800 \\
\hline $\mathrm{N}$ & -1.99247400 & 0.39840000 & -0.62718000 \\
\hline $\mathrm{H}$ & -1.38658000 & 2.09016000 & -1.27031400 \\
\hline $\mathrm{C}$ & -6.11363900 & 0.60465300 & 1.09997100 \\
\hline $\mathrm{C}$ & -5.60443600 & -0.26920600 & 2.03267700 \\
\hline $\mathrm{C}$ & -4.28172000 & -0.33399700 & 2.54578800 \\
\hline $\mathrm{C}$ & -5.44116900 & 1.65895700 & 0.42392500 \\
\hline $\mathrm{C}$ & -3.19729400 & 0.47288200 & 2.25870700 \\
\hline $\mathrm{C}$ & -4.13675200 & 2.09088300 & 0.56019300 \\
\hline $\mathrm{C}$ & -3.09023800 & 1.52674500 & 1.32686700 \\
\hline $\mathrm{H}$ & -7.16328400 & 0.48220900 & 0.84652400 \\
\hline $\mathrm{H}$ & -6.29764600 & -1.00398100 & 2.43373800 \\
\hline $\mathrm{H}$ & -4.11216800 & -1.10751100 & 3.29056500 \\
\hline $\mathrm{H}$ & -6.05611400 & 2.22890300 & -0.26798300 \\
\hline $\mathrm{H}$ & -2.28494900 & 0.24749100 & 2.80650300 \\
\hline $\mathrm{H}$ & -3.85829100 & 2.94889200 & -0.04329300 \\
\hline $\mathrm{H}$ & -2.15331300 & 2.07089100 & 1.32840300 \\
\hline \multicolumn{4}{|l|}{$C^{\prime}$} \\
\hline $\mathrm{Au}$ & -0.10169700 & -0.06503600 & -0.10373800 \\
\hline $\mathrm{P}$ & 2.02919100 & -1.02469400 & 0.09284500 \\
\hline $\mathrm{C}$ & 2.24140000 & -2.14124900 & -1.45319700 \\
\hline $\mathrm{C}$ & 2.05662000 & -1.95587900 & 1.78016500 \\
\hline $\mathrm{C}$ & 3.45824900 & 0.15164100 & 0.10469600 \\
\hline $\mathrm{C}$ & 3.35037200 & 1.55787600 & -0.03295200 \\
\hline $\mathrm{C}$ & 4.74179200 & -0.40301500 & 0.29487200 \\
\hline $\mathrm{C}$ & 4.52273600 & 2.33342900 & 0.02718800 \\
\hline $\mathrm{C}$ & 2.09400800 & 2.35006200 & -0.24242600 \\
\hline $\mathrm{C}$ & 5.88844200 & 0.38311300 & 0.35103900 \\
\hline $\mathrm{H}$ & 4.85602300 & -1.47279500 & 0.39996800 \\
\hline $\mathrm{C}$ & 5.77836700 & 1.76515700 & 0.21626600 \\
\hline $\mathrm{H}$ & 4.43215300 & 3.40974500 & -0.08067500 \\
\hline $\mathrm{C}$ & 1.63064800 & 2.62375800 & -1.53826800 \\
\hline $\mathrm{C}$ & 1.47831100 & 2.99201400 & 0.84523600 \\
\hline
\end{tabular}




\begin{tabular}{|c|c|c|c|}
\hline $\mathrm{H}$ & 6.85647000 & -0.08487600 & 0.49912000 \\
\hline $\mathrm{H}$ & 6.65998900 & 2.39712100 & 0.25704000 \\
\hline $\mathrm{C}$ & 0.57658400 & 3.52023800 & -1.74363600 \\
\hline $\mathrm{H}$ & 2.11784400 & 2.16154000 & -2.39128600 \\
\hline $\mathrm{C}$ & 0.42325100 & 3.88410800 & 0.64307500 \\
\hline $\mathrm{H}$ & 1.84853900 & 2.81050900 & 1.85000600 \\
\hline $\mathrm{C}$ & -0.02757800 & 4.15622200 & -0.65324500 \\
\hline $\mathrm{H}$ & 0.25031100 & 3.74423500 & -2.75522700 \\
\hline $\mathrm{H}$ & -0.02506100 & 4.39051300 & 1.49288500 \\
\hline $\mathrm{H}$ & -0.82496900 & 4.87557800 & -0.81494500 \\
\hline $\mathrm{C}$ & 1.15243400 & -3.23348600 & -1.43535200 \\
\hline $\mathrm{H}$ & 0.14777600 & -2.81811900 & -1.30939700 \\
\hline $\mathrm{H}$ & 1.17235100 & -3.76205900 & -2.39469000 \\
\hline $\mathrm{H}$ & 1.32505200 & -3.97787000 & -0.65342800 \\
\hline $\mathrm{C}$ & 2.02053700 & -1.22054300 & -2.67445300 \\
\hline $\mathrm{H}$ & 1.01679200 & -0.78541900 & -2.68866300 \\
\hline $\mathrm{H}$ & 2.75387900 & -0.40927200 & -2.71090900 \\
\hline $\mathrm{H}$ & 2.14024400 & -1.81441000 & -3.58728000 \\
\hline $\mathrm{C}$ & 3.62678500 & -2.80259900 & -1.59505200 \\
\hline $\mathrm{H}$ & 3.88789500 & -3.44245600 & -0.74978500 \\
\hline $\mathrm{H}$ & 3.60810800 & -3.43817600 & -2.48726600 \\
\hline $\mathrm{H}$ & 4.42070000 & -2.06753500 & -1.73992900 \\
\hline $\mathrm{C}$ & 0.66827400 & -2.58973000 & 2.02364500 \\
\hline $\mathrm{H}$ & -0.12043800 & -1.83428500 & 2.07603600 \\
\hline $\mathrm{H}$ & 0.39492100 & -3.31998100 & 1.25915600 \\
\hline $\mathrm{H}$ & 0.69132800 & -3.11370200 & 2.98576000 \\
\hline $\mathrm{C}$ & 3.11855400 & -3.06693500 & 1.89689800 \\
\hline $\mathrm{H}$ & 4.13993200 & -2.69929300 & 1.78704700 \\
\hline $\mathrm{H}$ & 3.04695200 & -3.50436900 & 2.89896900 \\
\hline $\mathrm{H}$ & 2.95499600 & -3.87627500 & 1.18128900 \\
\hline $\mathrm{C}$ & 2.30118000 & -0.88544000 & 2.86543200 \\
\hline $\mathrm{H}$ & 3.30477600 & -0.45831400 & 2.80919100 \\
\hline $\mathrm{H}$ & 1.57443100 & -0.06820300 & 2.80194200 \\
\hline $\mathrm{H}$ & 2.18857500 & -1.35182800 & 3.85034000 \\
\hline $\mathrm{C}$ & -3.04712800 & -0.38776900 & -0.90903400 \\
\hline $\mathrm{C}$ & -4.00657400 & -0.11323300 & -1.88756600 \\
\hline $\mathrm{C}$ & -2.91736300 & -1.68082600 & -0.39309000 \\
\hline $\mathrm{C}$ & -4.83022700 & -1.14185500 & -2.34758100 \\
\hline $\mathrm{H}$ & -4.09816100 & 0.88472000 & -2.29323700 \\
\hline $\mathrm{C}$ & -3.75504100 & -2.69764600 & -0.85124500 \\
\hline $\mathrm{H}$ & -2.17112400 & -1.89190600 & 0.36412000 \\
\hline $\mathrm{C}$ & -4.71237000 & -2.43254900 & -1.83142300 \\
\hline $\mathrm{H}$ & -5.56722400 & -0.92722200 & -3.11527200 \\
\hline $\mathrm{H}$ & -3.65007300 & -3.69934700 & -0.44583800 \\
\hline $\mathrm{H}$ & -5.35553600 & -3.22755200 & -2.19554200 \\
\hline $\mathrm{O}$ & -2.12651000 & 1.68143500 & -1.4326970 \\
\hline
\end{tabular}




\begin{tabular}{|c|c|c|c|}
\hline $\mathrm{N}$ & -2.16802000 & 0.67316600 & -0.39700900 \\
\hline $\mathrm{H}$ & -1.31479900 & 2.18769600 & -1.25143100 \\
\hline $\mathrm{C}$ & -5.62365500 & 0.24295800 & 1.55582900 \\
\hline $\mathrm{C}$ & -4.82092600 & -0.52210100 & 2.34255000 \\
\hline $\mathrm{C}$ & -3.45051700 & -0.25345200 & 2.69973000 \\
\hline $\mathrm{C}$ & -5.28144900 & 1.48838900 & 0.91399700 \\
\hline $\mathrm{C}$ & -2.55902400 & 0.58945700 & 2.12481700 \\
\hline $\mathrm{C}$ & -4.06369500 & 2.02717500 & 0.67048300 \\
\hline $\mathrm{C}$ & -2.71422800 & 1.41791000 & 0.88734200 \\
\hline $\mathrm{H}$ & -6.66285600 & -0.06225300 & 1.46209700 \\
\hline $\mathrm{H}$ & -5.27308000 & -1.38397000 & 2.82690700 \\
\hline $\mathrm{H}$ & -3.09561600 & -0.79091900 & 3.57826500 \\
\hline $\mathrm{H}$ & -6.13536300 & 2.08839500 & 0.60351600 \\
\hline $\mathrm{H}$ & -1.59690100 & 0.69887800 & 2.62167500 \\
\hline $\mathrm{H}$ & -4.04547300 & 3.01921100 & 0.22785600 \\
\hline $\mathrm{H}$ & -1.98158400 & 2.22768500 & 0.94646900 \\
\hline \multicolumn{4}{|l|}{$\mathrm{C}$} \\
\hline $\mathrm{C}$ & 3.03322300 & -1.61446400 & 0.64255600 \\
\hline $\mathrm{C}$ & 3.68865200 & -0.41861300 & 0.75189800 \\
\hline $\mathrm{C}$ & 3.41802200 & 0.76373500 & -0.03173600 \\
\hline $\mathrm{C}$ & 1.96154900 & -1.89955700 & -0.28192100 \\
\hline $\mathrm{C}$ & 2.20849300 & 1.10554800 & -0.52818000 \\
\hline $\mathrm{C}$ & 1.05981200 & -0.99886200 & -0.73316800 \\
\hline $\mathrm{C}$ & 0.96235400 & 0.37127200 & -0.11472800 \\
\hline $\mathrm{H}$ & 3.44490100 & -2.46056000 & 1.18985200 \\
\hline $\mathrm{H}$ & 4.57762100 & -0.39451900 & 1.37980800 \\
\hline $\mathrm{H}$ & 4.27137400 & 1.39986500 & -0.26051500 \\
\hline $\mathrm{H}$ & 1.91697700 & -2.91433200 & -0.67398500 \\
\hline $\mathrm{H}$ & 2.09923300 & 1.93264200 & -1.22333300 \\
\hline $\mathrm{H}$ & 0.38655400 & -1.25259200 & -1.54567700 \\
\hline $\mathrm{H}$ & 0.96006000 & 0.26584400 & 0.98455400 \\
\hline $\mathrm{N}$ & -0.24706100 & 1.09804500 & -0.55655100 \\
\hline $\mathrm{O}$ & -0.18888000 & 2.36049000 & 0.16095500 \\
\hline $\mathrm{H}$ & -0.42300600 & 2.99731500 & -0.52864300 \\
\hline $\mathrm{C}$ & -1.48085000 & 0.43920100 & -0.19071500 \\
\hline $\mathrm{C}$ & -2.17685500 & -0.24628000 & -1.19015000 \\
\hline $\mathrm{C}$ & -1.99434000 & 0.47905400 & 1.11198400 \\
\hline $\mathrm{C}$ & -3.36373500 & -0.91554500 & -0.88578300 \\
\hline $\mathrm{H}$ & -1.78637200 & -0.23452800 & -2.20289400 \\
\hline $\mathrm{C}$ & -3.18740500 & -0.17768800 & 1.40683900 \\
\hline $\mathrm{H}$ & -1.46745800 & 1.04373000 & 1.87277800 \\
\hline $\mathrm{C}$ & -3.87173200 & -0.88278900 & 0.41280700 \\
\hline $\mathrm{H}$ & -3.89743700 & -1.44788000 & -1.66774500 \\
\hline $\mathrm{H}$ & -3.58466600 & -0.14134800 & 2.41728800 \\
\hline $\mathrm{H}$ & -4.80045400 & -1.39393400 & 0.64851600 \\
\hline
\end{tabular}




\begin{tabular}{|c|c|c|c|}
\hline \multicolumn{4}{|c|}{ nitrosobenzene } \\
\hline $\mathrm{N}$ & 1.95029900 & 0.58868000 & -0.00013700 \\
\hline $\mathrm{O}$ & 2.72615600 & -0.35633800 & 0.00023200 \\
\hline $\mathrm{C}$ & 0.55504900 & 0.22689200 & -0.00016200 \\
\hline $\mathrm{C}$ & -0.33990100 & 1.30121200 & -0.00018200 \\
\hline $\mathrm{C}$ & 0.09721300 & -1.09834200 & 0.00001100 \\
\hline $\mathrm{C}$ & -1.71119200 & 1.05261200 & 0.00024200 \\
\hline $\mathrm{H}$ & 0.06204500 & 2.30970200 & -0.00021000 \\
\hline $\mathrm{C}$ & -1.27021000 & -1.33939600 & -0.00012100 \\
\hline $\mathrm{H}$ & 0.82812700 & -1.89971600 & 0.00004100 \\
\hline $\mathrm{C}$ & -2.17261100 & -0.26564300 & 0.00001200 \\
\hline $\mathrm{H}$ & -2.41677000 & 1.87742400 & 0.00044300 \\
\hline $\mathrm{H}$ & -1.64406500 & -2.35890500 & -0.00011200 \\
\hline $\mathrm{H}$ & -3.24076700 & -0.46257100 & 0.00014100 \\
\hline \multicolumn{4}{|l|}{ D } \\
\hline $\mathrm{C}$ & 3.38620300 & -1.51022300 & 0.34111300 \\
\hline $\mathrm{C}$ & 3.89534000 & -0.21178300 & 0.75476200 \\
\hline $\mathrm{C}$ & 3.35644400 & 0.93728200 & 0.29109300 \\
\hline $\mathrm{C}$ & 2.35220400 & -1.62002800 & -0.52248500 \\
\hline $\mathrm{C}$ & 2.10734800 & 0.94562000 & -0.48669400 \\
\hline $\mathrm{C}$ & 1.55963600 & -0.44867700 & -0.92734800 \\
\hline $\mathrm{C}$ & 0.84526500 & 0.38814000 & 0.11525200 \\
\hline $\mathrm{H}$ & 3.93203800 & -2.39787000 & 0.64854500 \\
\hline $\mathrm{H}$ & 4.79838100 & -0.18203800 & 1.35792200 \\
\hline $\mathrm{H}$ & 3.84925500 & 1.88948400 & 0.46737800 \\
\hline $\mathrm{H}$ & 2.10022600 & -2.58136800 & -0.96233400 \\
\hline $\mathrm{H}$ & 1.95889200 & 1.77468900 & -1.17331900 \\
\hline $\mathrm{H}$ & 1.05173600 & -0.49451000 & -1.88656700 \\
\hline $\mathrm{H}$ & 0.90211300 & 0.04739400 & 1.15000800 \\
\hline $\mathrm{N}$ & -0.37798000 & 1.01050900 & -0.30324000 \\
\hline $\mathrm{O}$ & -0.45442400 & 2.33896000 & 0.25410400 \\
\hline $\mathrm{H}$ & -0.35501400 & 2.90379300 & -0.52560500 \\
\hline $\mathrm{C}$ & -1.58870400 & 0.30292800 & -0.07036600 \\
\hline $\mathrm{C}$ & -1.59414300 & -1.09923000 & 0.01748400 \\
\hline $\mathrm{C}$ & -2.80893800 & 0.99361700 & 0.00230600 \\
\hline $\mathrm{C}$ & -2.79967600 & -1.78668000 & 0.15833800 \\
\hline $\mathrm{H}$ & -0.66450900 & -1.65301500 & -0.02322000 \\
\hline $\mathrm{C}$ & -4.00288300 & 0.29318700 & 0.15071100 \\
\hline $\mathrm{H}$ & -2.80687600 & 2.07494800 & -0.03917100 \\
\hline $\mathrm{C}$ & -4.01157500 & -1.10100600 & 0.22586600 \\
\hline $\mathrm{H}$ & -2.78264400 & -2.87100100 & 0.22467900 \\
\hline $\mathrm{H}$ & -4.93567200 & 0.84709600 & 0.21061600 \\
\hline $\mathrm{H}$ & -4.94579400 & -1.64171800 & 0.34071800 \\
\hline
\end{tabular}




$\begin{array}{crrr}\mathbf{E} & & & \\ \mathrm{C} & -3.68480400 & 1.20441400 & -0.47526800 \\ \mathrm{C} & -3.76810600 & 0.76604500 & 0.90997900 \\ \mathrm{C} & -3.05200200 & -0.28747800 & 1.36179800 \\ \mathrm{C} & -2.88841000 & 0.57467700 & -1.36808000 \\ \mathrm{C} & -2.02370200 & -0.93509400 & 0.53224800 \\ \mathrm{C} & -1.93516100 & -0.46275000 & -0.94986500 \\ \mathrm{C} & -0.84210900 & -0.13256300 & 0.04644300 \\ \mathrm{H} & -4.36119900 & 1.98614200 & -0.80913600 \\ \mathrm{H} & -4.50409700 & 1.23753600 & 1.55506000 \\ \mathrm{H} & -3.24375200 & -0.70428300 & 2.34677700 \\ \mathrm{H} & -2.95691500 & 0.80373900 & -2.42806900 \\ \mathrm{H} & -1.78330700 & -1.97723000 & 0.71913400 \\ \mathrm{H} & -1.63615800 & -1.21551600 & -1.67414300 \\ \mathrm{H} & -0.75408500 & 0.88671300 & 0.39382200 \\ \mathrm{~N} & 0.41109200 & -0.83516400 & -0.09526500 \\ \mathrm{O} & 0.35704300 & -2.09716500 & -0.32930700 \\ \mathrm{C} & 1.66472200 & -0.19057400 & -0.01321100 \\ \mathrm{C} & 1.79683900 & 1.20934700 & 0.03767400 \\ \mathrm{C} & 2.81661000 & -0.99868100 & 0.00778900 \\ \mathrm{C} & 3.06515300 & 1.77915900 & 0.12284700 \\ \mathrm{H} & 0.93161600 & 1.85866400 & -0.01195500 \\ \mathrm{C} & 4.07228600 & -0.41092500 & 0.09315400 \\ \mathrm{H} & 2.69144100 & -2.07231700 & -0.04242500 \\ \mathrm{C} & 4.20872200 & 0.97959300 & 0.15448900 \\ \mathrm{H} & 3.15572200 & 2.86080400 & 0.15756600 \\ \mathrm{H} & 4.95395600 & -1.04486100 & 0.11330400 \\ \mathrm{H} & 5.19256900 & 1.43305900 & 0.22138700\end{array}$

int2

$\begin{array}{lrrr}\mathrm{Au} & 0.37834800 & -0.28083800 & -0.00975500 \\ \mathrm{P} & -1.85919100 & -0.88470600 & 0.01879800 \\ \mathrm{C} & -2.18163600 & -1.77922200 & 1.68225500 \\ \mathrm{C} & -2.16545900 & -1.94530100 & -1.55703100 \\ \mathrm{C} & -3.00324400 & 0.56504600 & -0.05030800 \\ \mathrm{C} & -2.57864400 & 1.91803700 & -0.04896600 \\ \mathrm{C} & -4.38568300 & 0.29357000 & -0.12651800 \\ \mathrm{C} & -3.55555000 & 2.92723600 & -0.11878000 \\ \mathrm{C} & -1.16188700 & 2.40559600 & 0.01288800 \\ \mathrm{C} & -5.33346100 & 1.30981200 & -0.19761500 \\ \mathrm{H} & -4.73699500 & -0.72923300 & -0.12807700 \\ \mathrm{C} & -4.91479800 & 2.63875900 & -0.19233500 \\ \mathrm{H} & -3.22485500 & 3.96107100 & -0.11594100 \\ \mathrm{C} & -0.55935000 & 2.69862300 & 1.24719200 \\ \mathrm{C} & -0.47486500 & 2.72847100 & -1.16962600 \\ \mathrm{H} & -6.38819500 & 1.06054500 & -0.25604000\end{array}$




\begin{tabular}{|c|c|c|c|}
\hline $\mathrm{H}$ & -5.63881900 & 3.44579700 & -0.24615000 \\
\hline $\mathrm{C}$ & 0.70648900 & 3.28733200 & 1.29714300 \\
\hline $\mathrm{H}$ & -1.09362500 & 2.48505100 & 2.16814700 \\
\hline $\mathrm{C}$ & 0.79089600 & 3.31708200 & -1.11729000 \\
\hline $\mathrm{H}$ & -0.94489800 & 2.53677800 & -2.12989300 \\
\hline $\mathrm{C}$ & 1.38268800 & 3.59912500 & 0.11575600 \\
\hline $\mathrm{H}$ & 1.15418200 & 3.51818200 & 2.25914900 \\
\hline $\mathrm{H}$ & 1.30428200 & 3.57021000 & -2.04006600 \\
\hline $\mathrm{H}$ & 2.35805500 & 4.07455500 & 0.15611500 \\
\hline $\mathrm{C}$ & -1.33882500 & -3.06932000 & 1.73067200 \\
\hline $\mathrm{H}$ & -0.28486600 & -2.88091200 & 1.50251200 \\
\hline $\mathrm{H}$ & -1.39058600 & -3.48469700 & 2.74295600 \\
\hline $\mathrm{H}$ & -1.71273700 & -3.83579900 & 1.04683000 \\
\hline $\mathrm{C}$ & -1.70237700 & -0.81270100 & 2.78813200 \\
\hline $\mathrm{H}$ & -0.63313700 & -0.59435400 & 2.71142600 \\
\hline $\mathrm{H}$ & -2.25141900 & 0.13324700 & 2.76952700 \\
\hline $\mathrm{H}$ & -1.88117300 & -1.27978000 & 3.76288200 \\
\hline $\mathrm{C}$ & -3.66119200 & -2.12164500 & 1.94976700 \\
\hline $\mathrm{H}$ & -4.09770000 & -2.77036700 & 1.18785500 \\
\hline $\mathrm{H}$ & -3.71730800 & -2.65887200 & 2.90292200 \\
\hline $\mathrm{H}$ & -4.27833700 & -1.22655300 & 2.04694500 \\
\hline $\mathrm{C}$ & -0.95393500 & -2.87825000 & -1.78033400 \\
\hline $\mathrm{H}$ & -0.02872600 & -2.31306200 & -1.92410000 \\
\hline $\mathrm{H}$ & -0.80298400 & -3.58272200 & -0.95987800 \\
\hline $\mathrm{H}$ & -1.13158700 & -3.46348700 & -2.68927500 \\
\hline $\mathrm{C}$ & -3.44095200 & -2.81014400 & -1.51477500 \\
\hline $\mathrm{H}$ & -4.35394200 & -2.22316400 & -1.40217200 \\
\hline $\mathrm{H}$ & -3.52355700 & -3.34333800 & -2.46828100 \\
\hline $\mathrm{H}$ & -3.40672600 & -3.56612100 & -0.72648900 \\
\hline $\mathrm{C}$ & -2.24468700 & -0.95501100 & -2.73880700 \\
\hline $\mathrm{H}$ & -3.12959500 & -0.31695800 & -2.68749800 \\
\hline $\mathrm{H}$ & -1.35822000 & -0.31409400 & -2.79238600 \\
\hline $\mathrm{H}$ & -2.29520600 & -1.52710100 & -3.67163300 \\
\hline $\mathrm{C}$ & 4.75104600 & -0.50990100 & -0.01863200 \\
\hline $\mathrm{C}$ & 5.23481300 & 0.81052900 & -0.08151100 \\
\hline $\mathrm{C}$ & 5.63245200 & -1.60883900 & 0.02528600 \\
\hline $\mathrm{C}$ & 6.60787600 & 1.01598600 & -0.10005100 \\
\hline $\mathrm{H}$ & 4.53285700 & 1.63438100 & -0.11388500 \\
\hline $\mathrm{C}$ & 7.00140500 & -1.37804600 & 0.00571200 \\
\hline $\mathrm{H}$ & 5.24302900 & -2.62257500 & 0.07375700 \\
\hline $\mathrm{C}$ & 7.49400100 & -0.06880200 & -0.05691800 \\
\hline $\mathrm{H}$ & 6.99514200 & 2.02856700 & -0.14855000 \\
\hline $\mathrm{H}$ & 7.68741200 & -2.21789100 & 0.03919400 \\
\hline $\mathrm{H}$ & 8.56479300 & 0.10568000 & -0.07204400 \\
\hline $\mathrm{O}$ & 2.49987900 & 0.18343100 & -0.03479500 \\
\hline $\mathrm{N}$ & 3.39049200 & -0.76486700 & $0.0016050 \mathrm{C}$ \\
\hline
\end{tabular}


0.04605800

$\begin{array}{lrrr}\text { int3 } & & & \\ \mathrm{N} & 1.88333700 & -0.47845300 & 0.00007800 \\ \mathrm{O} & 2.78517100 & 0.42512000 & 0.00018500 \\ \mathrm{C} & 0.51071200 & -0.22103800 & 0.00000400 \\ \mathrm{C} & -0.39090200 & -1.29734200 & -0.00003300 \\ \mathrm{C} & 0.04352700 & 1.10237700 & -0.00000600 \\ \mathrm{C} & -1.75894500 & -1.04425100 & -0.00007900 \\ \mathrm{H} & -0.01853900 & -2.31906400 & -0.00001700 \\ \mathrm{C} & -1.32721400 & 1.33512100 & -0.00007200 \\ \mathrm{H} & 0.76701500 & 1.90848600 & 0.00002800 \\ \mathrm{C} & -2.23481600 & 0.27023200 & -0.00011200 \\ \mathrm{H} & -2.45646300 & -1.87643400 & -0.00010700 \\ \mathrm{H} & -1.69275000 & 2.35777700 & -0.00008800 \\ \mathrm{H} & -3.30295900 & 0.46258000 & -0.00016000 \\ \mathrm{H} & 2.18480000 & -1.45572000 & 0.00011200\end{array}$

3

$\mathrm{C}$

C

C

$\mathrm{C}$

$\mathrm{C}$

C

C

$\mathrm{H}$

$\mathrm{H}$

$\mathrm{H}$

$\mathrm{H}$

$\mathrm{H}$

$\mathrm{H}$

$\mathrm{N}$

$\mathrm{O}$

C

$\mathrm{C}$

C

C

$\mathrm{H}$

C

$\mathrm{H}$

C

$\mathrm{H}$

$\mathrm{H}$

$\mathrm{H}$

$-4.07259100$

1.27936600

0.31157600

$-4.69619100$

$0.06401900 \quad 0.01371600$

$-3.91701400-1.06323300$

$-0.25255800$

$-2.68634000$

1.35962500

0.33958600

$-2.52584800$

$-0.99601400$

$-0.22463300$

$-1.88434700$

0.22701400

0.07177400

$-0.45130400$

0.41705900

0.12009200

$-4.66869700$

2.16272200

0.52100200

$-5.78001200$

$-0.00167300$

$-0.00882300$

$-4.39702700$

$-2.00990500$

$-0.48358700$

$-2.20668900$

2.30769500

0.57034500

$-1.91674900-1.86561600$

$-0.42574900$

$-0.07475900$

1.39978800

0.36879500

0.47667800

$-0.50262100$

$-0.09143300$

0.24848900

$-1.74391600-0.31169600$

1.88526500

$-0.12340800$

$-0.04351300$

2.78356800

$-1.08058500$

0.42841400

2.33003000

1.12332700

$-0.48723000$

4.13882600

$-0.76744900$

0.49485000

2.39927600

$-2.04860300$

0.72464200

3.69099500

1.42312300

$-0.42087600$

1.63454900

1.83954200

$-0.91040200$

4.59626100

0.48410000

0.07499700

4.84036400

$-1.50524600$

0.87198500

4.04259100

2.38784700

$-0.77350900$

5.65463700

0.72146800

0.12172600 


$\begin{array}{lrrr}\mathbf{6} & & & \\ \mathrm{N} & -1.82663700 & -0.60679200 & -0.21597600 \\ \mathrm{H} & -2.05431400 & -1.43734500 & 0.32614900 \\ \mathrm{O} & -2.70680300 & 0.41504900 & 0.25813200 \\ \mathrm{H} & -3.20592600 & 0.64906300 & -0.53726100 \\ \mathrm{C} & -0.46019300 & -0.26406400 & -0.06884400 \\ \mathrm{C} & -0.02778400 & 1.06688300 & -0.07126900 \\ \mathrm{C} & 0.48468100 & -1.29957200 & -0.00432700 \\ \mathrm{C} & 1.33657900 & 1.35060400 & -0.01394000 \\ \mathrm{H} & -0.76295300 & 1.86148000 & -0.09957600 \\ \mathrm{C} & 1.84376800 & -1.00195600 & 0.04681300 \\ \mathrm{H} & 0.15148400 & -2.33482300 & -0.00222800 \\ \mathrm{C} & 2.28069100 & 0.32488400 & 0.04251300 \\ \mathrm{H} & 1.66104700 & 2.38751300 & -0.01006800 \\ \mathrm{H} & 2.56435200 & -1.81334500 & 0.09707900 \\ \mathrm{H} & 3.34074900 & 0.55393900 & 0.08700300\end{array}$

TS $_{1}$

$\begin{array}{cccc}\mathrm{Au} & -0.16583300 & 0.03959500 & 0.10555100 \\ \mathrm{P} & 2.03258200 & 0.79350800 & 0.46402500 \\ \mathrm{C} & 2.33955200 & 0.77936500 & 2.35752100 \\ \mathrm{C} & 2.16777400 & 2.52566900 & -0.36687300 \\ \mathrm{C} & 3.35528200 & -0.26306400 & -0.28720100 \\ \mathrm{C} & 3.10990500 & -1.47436300 & -0.98189100 \\ \mathrm{C} & 4.68783400 & 0.18923500 & -0.18174800 \\ \mathrm{C} & 4.20318200 & -2.16420800 & -1.53721400 \\ \mathrm{C} & 1.77725700 & -2.12625800 & -1.19783300 \\ \mathrm{C} & 5.75367800 & -0.51080500 & -0.73842800 \\ \mathrm{H} & 4.90621800 & 1.10626300 & 0.34736300 \\ \mathrm{C} & 5.50914000 & -1.69857200 & -1.42437900 \\ \mathrm{H} & 4.00683400 & -3.09100200 & -2.06705800 \\ \mathrm{C} & 1.27029900 & -3.04212400 & -0.26203700 \\ \mathrm{C} & 1.10354700 & -1.96313100 & -2.41891900 \\ \mathrm{H} & 6.76389400 & -0.12742700 & -0.63479700 \\ \mathrm{H} & 6.32636500 & -2.25970500 & -1.86693400 \\ \mathrm{C} & 0.11050100 & -3.76922100 & -0.53761500 \\ \mathrm{H} & 1.80141400 & -3.20300000 & 0.67143800 \\ \mathrm{C} & -0.05820200 & -2.68812600 & -2.69104500 \\ \mathrm{H} & 1.50356000 & -1.27959000 & -3.16233300 \\ \mathrm{C} & -0.55517500 & -3.59461200 & -1.75260600 \\ \mathrm{H} & -0.26337400 & -4.48239400 & 0.19087400 \\ \mathrm{H} & -0.56537500 & -2.55431000 & -3.64168700 \\ \mathrm{H} & -1.45009500 & -4.16932300 & -1.97033900 \\ \mathrm{C} & 1.33820800 & 1.73753600 & 3.03402400 \\ \mathrm{H} & 0.30787700 & 1.54956700 & 2.71568400 \\ \mathrm{H} & 1.38581300 & 1.58970000 & 4.11868600\end{array}$




\begin{tabular}{|c|c|c|c|}
\hline $\mathrm{H}$ & 1.57704700 & 2.78691500 & 2.84078800 \\
\hline $\mathrm{C}$ & 2.05396500 & -0.66748000 & 2.81849800 \\
\hline $\mathrm{H}$ & 1.02860600 & -0.97744700 & 2.59755400 \\
\hline $\mathrm{H}$ & 2.73708200 & -1.38385200 & 2.35303500 \\
\hline $\mathrm{H}$ & 2.19932800 & -0.72738400 & 3.90295700 \\
\hline $\mathrm{C}$ & 3.77054700 & 1.14550700 & 2.79816300 \\
\hline $\mathrm{H}$ & 4.07852400 & 2.14176500 & 2.47486200 \\
\hline $\mathrm{H}$ & 3.80279700 & 1.13635700 & 3.89350700 \\
\hline $\mathrm{H}$ & 4.50627000 & 0.41912600 & 2.44818800 \\
\hline $\mathrm{C}$ & 0.86525000 & 3.30902200 & -0.09282100 \\
\hline $\mathrm{H}$ & -0.01591700 & 2.78117000 & -0.46677300 \\
\hline $\mathrm{H}$ & 0.71405900 & 3.51628600 & 0.96889100 \\
\hline $\mathrm{H}$ & 0.92681100 & 4.27286000 & -0.61141300 \\
\hline $\mathrm{C}$ & 3.35507600 & 3.39623000 & 0.08772600 \\
\hline $\mathrm{H}$ & 4.32485700 & 2.94188000 & -0.11993600 \\
\hline $\mathrm{H}$ & 3.32260000 & 4.33894200 & -0.47028600 \\
\hline $\mathrm{H}$ & 3.30340300 & 3.65057400 & 1.14925400 \\
\hline $\mathrm{C}$ & 2.26820600 & 2.26294400 & -1.88549500 \\
\hline $\mathrm{H}$ & 3.20802900 & 1.77892200 & -2.16027900 \\
\hline $\mathrm{H}$ & 1.44147200 & 1.64159100 & -2.24612300 \\
\hline $\mathrm{H}$ & 2.21712300 & 3.22219200 & -2.41272200 \\
\hline $\mathrm{C}$ & -2.84643400 & -1.55983800 & 0.53296100 \\
\hline $\mathrm{C}$ & -3.95940400 & -2.26207200 & 0.02372300 \\
\hline $\mathrm{C}$ & -2.42090900 & -1.79664800 & 1.85588500 \\
\hline $\mathrm{C}$ & -4.62563400 & -3.17661600 & 0.83204400 \\
\hline $\mathrm{H}$ & -4.26414200 & -2.09497500 & -1.00200300 \\
\hline $\mathrm{C}$ & -3.09433400 & -2.71657000 & 2.64983000 \\
\hline $\mathrm{H}$ & -1.57210100 & -1.24342600 & 2.24665800 \\
\hline $\mathrm{C}$ & -4.20106700 & -3.40944900 & 2.14496300 \\
\hline $\mathrm{H}$ & -5.47720600 & -3.72035600 & 0.43433100 \\
\hline $\mathrm{H}$ & -2.76202200 & -2.89237500 & 3.66830300 \\
\hline $\mathrm{H}$ & -4.72564800 & -4.12633500 & 2.76841400 \\
\hline $\mathrm{O}$ & -2.63695000 & -0.40239600 & -1.43247700 \\
\hline $\mathrm{N}$ & -2.14825900 & -0.62303500 & -0.23864300 \\
\hline $\mathrm{C}$ & -2.94661900 & 3.63817500 & 0.30078900 \\
\hline $\mathrm{C}$ & -3.01943600 & 4.08676600 & -0.99302100 \\
\hline $\mathrm{C}$ & -3.75009300 & 3.53093400 & -2.09289900 \\
\hline $\mathrm{C}$ & -3.59801100 & 2.50415100 & 0.88484000 \\
\hline $\mathrm{C}$ & -4.42410600 & 2.34304500 & -2.15258100 \\
\hline $\mathrm{C}$ & -4.31979700 & 1.51719100 & 0.26795100 \\
\hline $\mathrm{C}$ & -4.52878500 & 1.29486000 & -1.15238500 \\
\hline $\mathrm{H}$ & -2.36962800 & 4.24840100 & 0.99093500 \\
\hline $\mathrm{H}$ & -2.49344200 & 5.01365000 & -1.20929600 \\
\hline $\mathrm{H}$ & -3.77327700 & 4.14293200 & -2.99093600 \\
\hline $\mathrm{H}$ & -3.53051400 & 2.43904200 & 1.96822000 \\
\hline $\mathrm{H}$ & -4.92690000 & 2.12513400 & -3.0925030 \\
\hline
\end{tabular}




$\begin{array}{lrrr}H & -4.76060900 & 0.76611200 & 0.91885800 \\ H & -5.33570300 & 0.59116600 & -1.36232000 \\ H & -3.50574200 & 0.41734600 & -1.40119200\end{array}$

$\mathbf{T S}_{2}$

$\mathrm{Au}$

$\begin{array}{llll}-0.02699100 & -0.13128000 & -0.19103900\end{array}$

$\mathrm{P}$

$\begin{array}{llll}2.14306500 & -0.98945100 & 0.12195100\end{array}$

C

$\begin{array}{llll}2.46696900 & -2.12027500 & -1.39520400\end{array}$

C

$2.15510900-1.89533800$

1.82265800

C

$3.52591100 \quad 0.24247300$

0.16994200

C

$3.36092800 \quad 1.64230700$

0.02370100

$\begin{array}{llll}4.82776300 & -0.25357700 & 0.39387700\end{array}$

$\mathrm{C}$

4.49657300

2.46903800

0.10298900

2.07437200

2.37830600

$-0.20758900$

$\mathrm{C}$

5.93767400

$0.58223800 \quad 0.47072400$

$\mathrm{H}$

4.98637400

$-1.31651300$

0.50914500

5.77137000

1.95720100

0.32249700

4.36122900

$3.53988500-0.01256200$

$\mathrm{C}$

1.63038100

2.64901700

$-1.51056500$

1.40129700

2.97205200

0.87312000

6.92141700

0.15773500

0.64445500

6.62378800

2.62707000

0.37756600

0.54030000

$3.49704700-1.73024300$

$\mathrm{H}$

2.15888800

2.22122100

$-2.35690500$

0.30994300

3.81559200

0.65675800

$\begin{array}{lll}1.75513600 & 2.79300400 & 1.88416200\end{array}$

$\mathrm{H}$

$-0.12064500$

$4.08641000-0.64691100$

0.22688000

$3.71787700-2.74642200$

$\begin{array}{llll}-0.18142300 & 4.28892200 & 1.50203100\end{array}$

$\begin{array}{llll}-0.94444600 & 4.77316600 & -0.81915600\end{array}$

$\mathrm{H}$

1.42492700

$-3.25800500-1.39893200$

$\mathrm{H}$

$\begin{array}{llll}0.40067900 & -2.88504700 & -1.30089900\end{array}$

$\mathrm{H}$

$\begin{array}{llll}1.49219100 & -3.79116200 & -2.35363400\end{array}$

$\begin{array}{llll}1.60893300 & -3.98968700 & -0.60748300\end{array}$

$\mathrm{H}$

$\begin{array}{llll}2.25091900 & -1.22795600 & -2.63864900\end{array}$

$\mathrm{C}$

$\begin{array}{llll}1.22959700 & -0.84006000 & -2.69719500\end{array}$

$\mathrm{H}$

$2.94696300 \quad-0.38379200 \quad-2.66021900$

$\mathrm{H}$

$\begin{array}{llll}2.43277100 & -1.82819000 & -3.53704800\end{array}$

$\mathrm{C}$

$3.88189500 \quad-2.72553200-1.48025200$

$\mathrm{H} \quad 4.14323800 \quad-3.33435300 \quad-0.61237900$

$\mathrm{H} \quad 3.91774900 \quad-3.38142000 \quad-2.35723200$

$\mathrm{H} \quad 4.64879700 \quad-1.96113000 \quad-1.61879100$

C $\quad 0.79577000 \quad-2.60298900 \quad 2.01992700$

$\mathrm{H} \quad-0.03516200 \quad-1.89222800 \quad 2.00839400$

$\mathrm{H} \quad 0.60116200 \quad-3.36766800 \quad 1.26530800$

$\mathrm{H} \quad 0.80010400 \quad-3.09769100 \quad 2.99781200$ 


$\begin{array}{lrrr}\mathrm{C} & 3.27210000 & -2.94091800 & 2.00642900 \\ \mathrm{H} & 4.27413600 & -2.51491900 & 1.93572200 \\ \mathrm{H} & 3.18089600 & -3.36825200 & 3.01142900 \\ \mathrm{H} & 3.18844900 & -3.76807400 & 1.29750000 \\ \mathrm{C} & 2.29156300 & -0.79612000 & 2.89830800 \\ \mathrm{H} & 3.26892200 & -0.30914900 & 2.87229000 \\ \mathrm{H} & 1.51956800 & -0.02604300 & 2.79205400 \\ \mathrm{H} & 2.16991600 & -1.25317600 & 3.88667900 \\ \mathrm{C} & -2.96265800 & -0.52152800 & -0.95934600 \\ \mathrm{C} & -3.95658800 & -0.28588100 & -1.92899600 \\ \mathrm{C} & -2.92922200 & -1.76733900 & -0.29868600 \\ \mathrm{C} & -4.88545700 & -1.28286400 & -2.22470300 \\ \mathrm{H} & -3.97806200 & 0.65879700 & -2.45455200 \\ \mathrm{C} & -3.87082000 & -2.74639200 & -0.59902000 \\ \mathrm{H} & -2.15200600 & -1.96429700 & 0.43242800 \\ \mathrm{C} & -4.85830900 & -2.50991200 & -1.56008400 \\ \mathrm{H} & -5.63720700 & -1.09559400 & -2.98603400 \\ \mathrm{H} & -3.82745000 & -3.70346200 & -0.08718100 \\ \mathrm{H} & -5.58435500 & -3.28021400 & -1.79998400 \\ \mathrm{O} & -2.11705300 & 1.55340000 & -1.49204800 \\ \mathrm{~N} & -2.04998400 & 0.47488200 & -0.55761700 \\ \mathrm{H} & -1.37421900 & 2.12968800 & -1.24657500 \\ \mathrm{C} & -6.01425400 & 0.59133700 & 1.29836400 \\ \mathrm{C} & -5.43208500 & -0.27456900 & 2.18683000 \\ \mathrm{C} & -4.06996200 & -0.31737600 & 2.60745300 \\ \mathrm{C} & -5.40082600 & 1.66612400 & 0.58757000 \\ \mathrm{C} & -3.00706400 & 0.45040500 & 2.19914500 \\ \mathrm{C} & -4.08938800 & 2.07076200 & 0.57692400 \\ \mathrm{C} & -2.94941300 & 1.44882500 & 1.17944100 \\ \mathrm{H} & -7.08165900 & 0.47203100 & 1.13212500 \\ \mathrm{H} & -6.08676800 & -1.00740700 & 2.65126900 \\ \mathrm{H} & -3.85365900 & -1.04697100 & 3.38465100 \\ \mathrm{H} & -6.08443700 & 2.27340000 & -0.00127200 \\ \mathrm{H} & -2.06077300 & 0.25385300 & 2.69890300 \\ \mathrm{H} & -3.86972300 & 2.95386700 & -0.01447600 \\ \mathrm{H} & -2.04824900 & 2.05207700 & 1.18376800\end{array}$

$\mathrm{TS}_{3}$

$\begin{array}{lrrr}\mathrm{C} & -3.24232400 & -1.52444400 & -0.38529200 \\ \mathrm{C} & -3.74882700 & -0.28142100 & -0.83882400 \\ \mathrm{C} & -3.34568500 & 0.92885400 & -0.30112400 \\ \mathrm{C} & -2.30615500 & -1.62118000 & 0.63057400 \\ \mathrm{C} & -2.12131300 & 1.10415700 & 0.37635000 \\ \mathrm{C} & -1.44466000 & -0.56148400 & 0.98450400 \\ \mathrm{C} & -0.90056800 & 0.37411000 & -0.03776900 \\ \mathrm{H} & -3.77721700 & -2.42168100 & -0.68667100\end{array}$




$\begin{array}{lrrr}\mathrm{H} & -4.63986300 & -0.30209800 & -1.46122300 \\ \mathrm{H} & -4.03466200 & 1.76984800 & -0.32508300 \\ \mathrm{H} & -2.29019800 & -2.51686700 & 1.24729700 \\ \mathrm{H} & -1.98478700 & 1.94249800 & 1.05241700 \\ \mathrm{H} & -0.96607200 & -0.53448900 & 1.95895900 \\ \mathrm{H} & -0.92763100 & -0.00452500 & -1.06584400 \\ \mathrm{~N} & 0.34187300 & 1.01963200 & 0.34322000 \\ \mathrm{O} & 0.39666400 & 2.31985500 & -0.27533900 \\ \mathrm{H} & 0.32162300 & 2.92067500 & 0.47949100 \\ \mathrm{C} & 1.54807100 & 0.30761700 & 0.08796800 \\ \mathrm{C} & 1.55734400 & -1.09110600 & -0.03820600 \\ \mathrm{C} & 2.76635400 & 1.00355100 & 0.02735500 \\ \mathrm{C} & 2.76558100 & -1.77013400 & -0.20248300 \\ \mathrm{H} & 0.63254800 & -1.65340600 & -0.00632800 \\ \mathrm{C} & 3.96189400 & 0.31296400 & -0.14606700 \\ \mathrm{H} & 2.76193900 & 2.08363900 & 0.09755600 \\ \mathrm{C} & 3.97432500 & -1.07909700 & -0.25788400 \\ \mathrm{H} & 2.75152600 & -2.85224900 & -0.29793700 \\ \mathrm{H} & 4.89241800 & 0.87153900 & -0.19570900 \\ \mathrm{H} & 4.90972600 & -1.61347100 & -0.39137200\end{array}$

$\begin{array}{lrrr}\text { TS } & & & \\ \mathrm{Au} & -0.70872600 & -0.16994800 & -0.34528800 \\ \mathrm{P} & -2.92948200 & 0.44694000 & -0.77934000 \\ \mathrm{C} & -3.46586100 & -0.51960000 & -2.34722200 \\ \mathrm{C} & -2.94106800 & 2.36309100 & -0.97149900 \\ \mathrm{C} & -4.14949100 & 0.02602500 & 0.54631200 \\ \mathrm{C} & -3.82061000 & -0.66794500 & 1.73746500 \\ \mathrm{C} & -5.48368700 & 0.44730000 & 0.36290800 \\ \mathrm{C} & -4.83819000 & -0.90738600 & 2.67892100 \\ \mathrm{C} & -2.46770000 & -1.18617300 & 2.12241500 \\ \mathrm{C} & -6.47346700 & 0.20054800 & 1.30911700 \\ \mathrm{H} & -5.76368700 & 0.97762600 & -0.53667100 \\ \mathrm{C} & -6.14807600 & -0.48423000 & 2.47816800 \\ \mathrm{H} & -4.57869500 & -1.44093700 & 3.58790200 \\ \mathrm{C} & -2.07807100 & -2.49106500 & 1.78089300 \\ \mathrm{C} & -1.64482900 & -0.43490900 & 2.97678200 \\ \mathrm{H} & -7.48816600 & 0.54191700 & 1.12998500 \\ \mathrm{H} & -6.90585000 & -0.68754400 & 3.22852500 \\ \mathrm{C} & -0.88913100 & -3.02900600 & 2.27799500 \\ \mathrm{H} & -2.72093500 & -3.09332500 & 1.14584100 \\ \mathrm{C} & -0.45531200 & -0.97349600 & 3.47079900 \\ \mathrm{H} & -1.95091800 & 0.56522300 & 3.26981800 \\ \mathrm{C} & -0.07821700 & -2.27277600 & 3.12660300 \\ \mathrm{H} & -0.60567300 & -4.04326900 & 2.01375800 \\ \mathrm{H} & 0.16723800 & -0.38285200 & 4.13577000\end{array}$




\begin{tabular}{|c|c|c|c|}
\hline $\mathrm{H}$ & 0.83719900 & -2.69791000 & 3.52638500 \\
\hline $\mathrm{C}$ & -2.57305000 & -0.09163600 & -3.52969100 \\
\hline $\mathrm{H}$ & -1.50765900 & -0.14527600 & -3.28444300 \\
\hline $\mathrm{H}$ & -2.75619400 & -0.76814400 & -4.37188700 \\
\hline $\mathrm{H}$ & -2.79914400 & 0.92109800 & -3.87380700 \\
\hline $\mathrm{C}$ & -3.21682900 & -2.01181500 & -2.03216500 \\
\hline $\mathrm{H}$ & -2.16343800 & -2.22169100 & -1.82500500 \\
\hline $\mathrm{H}$ & -3.81084900 & -2.35183600 & -1.17870000 \\
\hline $\mathrm{H}$ & -3.51540300 & -2.60874200 & -2.90129100 \\
\hline $\mathrm{C}$ & -4.94713100 & -0.35494800 & -2.74140600 \\
\hline $\mathrm{H}$ & -5.22695000 & 0.68165300 & -2.93914900 \\
\hline $\mathrm{H}$ & -5.11747700 & -0.91800200 & -3.66590700 \\
\hline $\mathrm{H}$ & -5.62075900 & -0.76357700 & -1.98588900 \\
\hline $\mathrm{C}$ & -1.65687500 & 2.80001700 & -1.71213600 \\
\hline $\mathrm{H}$ & -0.75417800 & 2.52476800 & -1.16003800 \\
\hline $\mathrm{H}$ & -1.58331400 & 2.38267500 & -2.71846000 \\
\hline $\mathrm{H}$ & -1.66965500 & 3.89174200 & -1.80835400 \\
\hline $\mathrm{C}$ & -4.15377000 & 2.93865700 & -1.72900500 \\
\hline $\mathrm{H}$ & -5.10676700 & 2.71590400 & -1.24645500 \\
\hline $\mathrm{H}$ & -4.05807200 & 4.03011600 & -1.75032000 \\
\hline $\mathrm{H}$ & -4.19578000 & 2.60050700 & -2.76725100 \\
\hline $\mathrm{C}$ & -2.89889000 & 2.94230500 & 0.45907400 \\
\hline $\mathrm{H}$ & -3.81796600 & 2.74292300 & 1.01456700 \\
\hline $\mathrm{H}$ & -2.05432500 & 2.54630700 & 1.03283700 \\
\hline $\mathrm{H}$ & -2.77374300 & 4.02888400 & 0.39374400 \\
\hline $\mathrm{C}$ & 1.81795500 & -2.01756400 & -0.45897400 \\
\hline $\mathrm{C}$ & 2.52462300 & -2.78643200 & 0.46863700 \\
\hline $\mathrm{C}$ & 1.46570300 & -2.54355500 & -1.70668100 \\
\hline $\mathrm{C}$ & 2.86680200 & -4.09932900 & 0.14236500 \\
\hline $\mathrm{H}$ & 2.78546400 & -2.34812900 & 1.42386700 \\
\hline $\mathrm{C}$ & 1.82085100 & -3.85341100 & -2.02498500 \\
\hline $\mathrm{H}$ & 0.92106900 & -1.92973000 & -2.41968300 \\
\hline $\mathrm{C}$ & 2.51874900 & -4.63583000 & -1.10043800 \\
\hline $\mathrm{H}$ & 3.40344600 & -4.70796900 & 0.86429200 \\
\hline $\mathrm{H}$ & 1.55488100 & -4.26221400 & -2.99481900 \\
\hline $\mathrm{H}$ & 2.78892300 & -5.65763000 & -1.34748700 \\
\hline $\mathrm{O}$ & 1.99390600 & -0.12320400 & 0.90499500 \\
\hline $\mathrm{N}$ & 1.41639900 & -0.65529400 & -0.14841900 \\
\hline $\mathrm{C}$ & 7.29121700 & -1.03420500 & 0.46525800 \\
\hline $\mathrm{C}$ & 7.80003400 & 0.26947200 & 0.07294100 \\
\hline $\mathrm{C}$ & 7.07451100 & 1.11161300 & -0.70083800 \\
\hline $\mathrm{C}$ & 6.06796300 & -1.46317600 & 0.08003200 \\
\hline $\mathrm{C}$ & 5.68004200 & 0.81425800 & -1.04344900 \\
\hline $\mathrm{C}$ & 5.12930000 & -0.56814600 & -0.62116800 \\
\hline $\mathrm{C}$ & 4.64887700 & 0.66884000 & 0.08386100 \\
\hline $\mathrm{H}$ & 7.95740700 & -1.70623300 & 0.99798200 \\
\hline
\end{tabular}




$\begin{array}{lrrr}\mathrm{H} & 8.82420300 & 0.52096500 & 0.33153600 \\ \mathrm{H} & 7.51958800 & 2.01447000 & -1.10825100 \\ \mathrm{H} & 5.75479800 & -2.48873400 & 0.24870200 \\ \mathrm{H} & 5.26588600 & 1.25056900 & -1.94844800 \\ \mathrm{H} & 4.39617000 & -1.02476200 & -1.27897300 \\ \mathrm{H} & 4.98006900 & 0.85746600 & 1.09574200 \\ \mathrm{~N} & 3.32129800 & 1.16249300 & -0.17030800 \\ \mathrm{O} & 2.80581900 & 0.87403400 & -1.33103200 \\ \mathrm{H} & 1.90036800 & 0.06320400 & -1.01332200 \\ \mathrm{C} & 2.91948100 & 2.41075500 & 0.43050200 \\ \mathrm{C} & 3.10810800 & 2.61743300 & 1.80097300 \\ \mathrm{C} & 2.35590800 & 3.40070500 & -0.37914100 \\ \mathrm{C} & 2.73985300 & 3.83938900 & 2.35853200 \\ \mathrm{H} & 3.50196200 & 1.82621900 & 2.42852000 \\ \mathrm{C} & 1.99320900 & 4.61887300 & 0.19439300 \\ \mathrm{H} & 2.22926500 & 3.21673500 & -1.43884500 \\ \mathrm{C} & 2.18533200 & 4.84305200 & 1.55920100 \\ \mathrm{H} & 2.87965300 & 4.00415200 & 3.42210000 \\ \mathrm{H} & 1.57264400 & 5.39894000 & -0.43255000 \\ \mathrm{H} & 1.90570600 & 5.79499900 & 1.99925900\end{array}$

$\begin{array}{lrrr}\text { TS5 } & & & \\ \mathrm{C} & 3.11987000 & -0.57156300 & 0.45740100 \\ \mathrm{C} & 4.19023300 & 0.31596700 & 0.67674700 \\ \mathrm{C} & 3.30485200 & -1.72486100 & -0.33140500 \\ \mathrm{C} & 5.42893300 & 0.04024700 & 0.11150000 \\ \mathrm{H} & 4.01473500 & 1.19434900 & 1.28561600 \\ \mathrm{C} & 4.55104400 & -1.98177500 & -0.88826700 \\ \mathrm{H} & 2.46672600 & -2.39319300 & -0.50644200 \\ \mathrm{C} & 5.62035800 & -1.10486900 & -0.67065400 \\ \mathrm{H} & 6.25651800 & 0.72357800 & 0.27988600 \\ \mathrm{H} & 4.69125800 & -2.86781100 & -1.50057900 \\ \mathrm{H} & 6.59239300 & -1.31172300 & -1.10742300 \\ \mathrm{O} & 1.60302700 & 0.75769300 & 1.64000500 \\ \mathrm{~N} & 1.88602800 & -0.35525900 & 1.05475800 \\ \mathrm{H} & 1.06946900 & -0.87334300 & 0.66306900 \\ \mathrm{C} & -1.57629600 & 4.28312400 & 0.41643600 \\ \mathrm{C} & -1.97791200 & 4.24588900 & -0.96507700 \\ \mathrm{C} & -1.63069400 & 3.17157100 & -1.75195000 \\ \mathrm{C} & -0.90491100 & 3.25305800 & 0.98813500 \\ \mathrm{C} & -0.91072800 & 2.07809000 & -1.21688400 \\ \mathrm{C} & -0.55227900 & 2.02106000 & 0.22820700 \\ \mathrm{C} & -1.59666600 & 0.94881200 & 0.20524000 \\ \mathrm{H} & -1.80310700 & 5.16997100 & 1.00227900 \\ \mathrm{H} & -2.52507200 & 5.08342100 & -1.38523900 \\ \mathrm{H} & -1.88710500 & 3.16159300 & -2.80783300\end{array}$




$\begin{array}{lrrr}\mathrm{H} & -0.57604800 & 3.30609500 & 2.02181200 \\ \mathrm{H} & -0.51853400 & 1.29138100 & -1.85160800 \\ \mathrm{H} & 0.44633000 & 1.60866800 & 0.52800300 \\ \mathrm{H} & -2.61705800 & 1.18696400 & 0.46661400 \\ \mathrm{~N} & -1.31260100 & -0.34256100 & -0.06758700 \\ \mathrm{O} & -0.13929100 & -0.66278100 & -0.56616300 \\ \mathrm{C} & -2.29592800 & -1.37569700 & 0.02211200 \\ \mathrm{C} & -3.41777400 & -1.26293100 & 0.85720300 \\ \mathrm{C} & -2.09340000 & -2.54016200 & -0.72980700 \\ \mathrm{C} & -4.34473300 & -2.30137100 & 0.90573600 \\ \mathrm{H} & -3.55439700 & -0.39398200 & 1.49087800 \\ \mathrm{C} & -3.02542900 & -3.57175900 & -0.66459900 \\ \mathrm{H} & -1.21004100 & -2.60371800 & -1.35161500 \\ \mathrm{C} & -4.15733200 & -3.45798100 & 0.14594200 \\ \mathrm{H} & -5.20906300 & -2.21070600 & 1.55670200 \\ \mathrm{H} & -2.86745700 & -4.46931700 & -1.25519400 \\ \mathrm{H} & -4.88067300 & -4.26589200 & 0.19404600\end{array}$

\section{8. ${ }^{1} \mathrm{H}$ and ${ }^{13} \mathrm{C}$ spectra of key compounds:}


Solvent: $\mathrm{CDCl}_{3}$

SFO1: $400 \mathrm{MHz}$

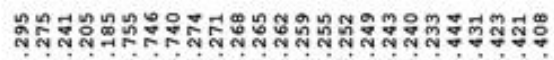

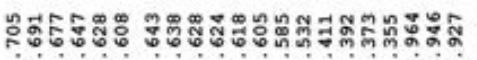

N

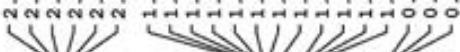

SAM-02-195 (4-n-Bu-CHT)

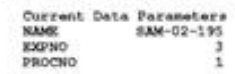

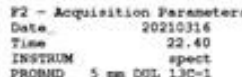

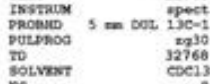

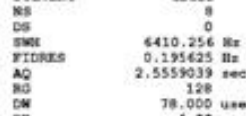

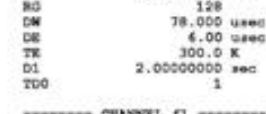

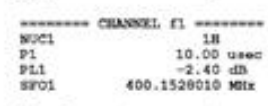

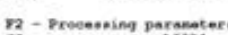

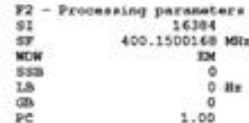

$$
\begin{aligned}
& \text { pe } \quad 1.00^{\circ}
\end{aligned}
$$
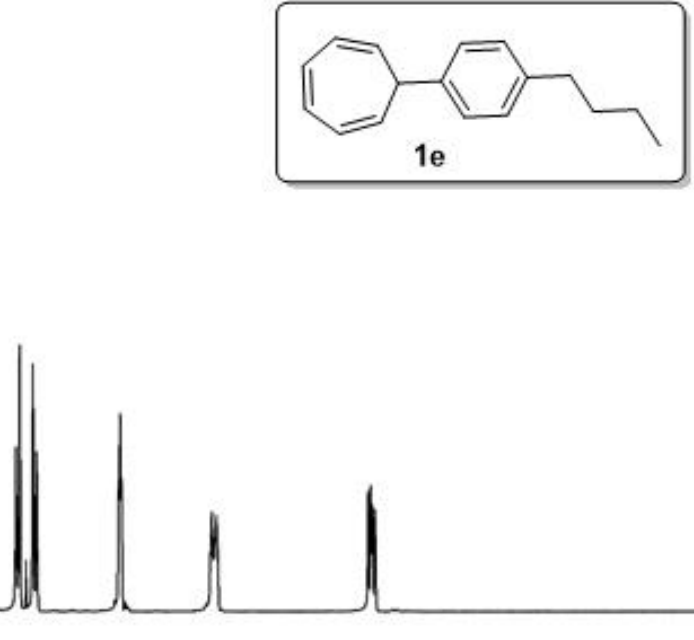

$\begin{array}{llll}9.5 & 9.0 & 8.5 & 8.0\end{array}$

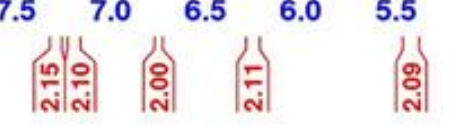
|ำ

4.54
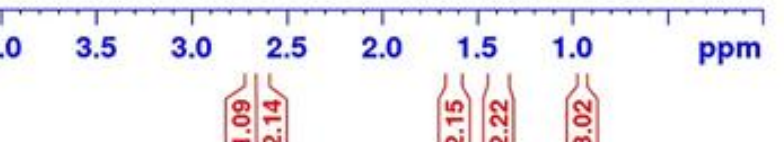
Solvent: $\mathrm{CDCl}_{3}$

SFO1: $100 \mathrm{MHz}$

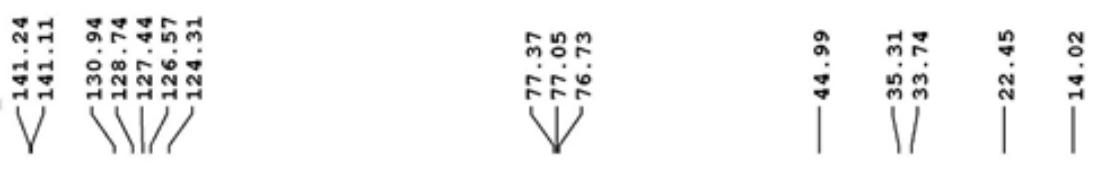

SAM-02-195 (4-n-Bu-CHT)
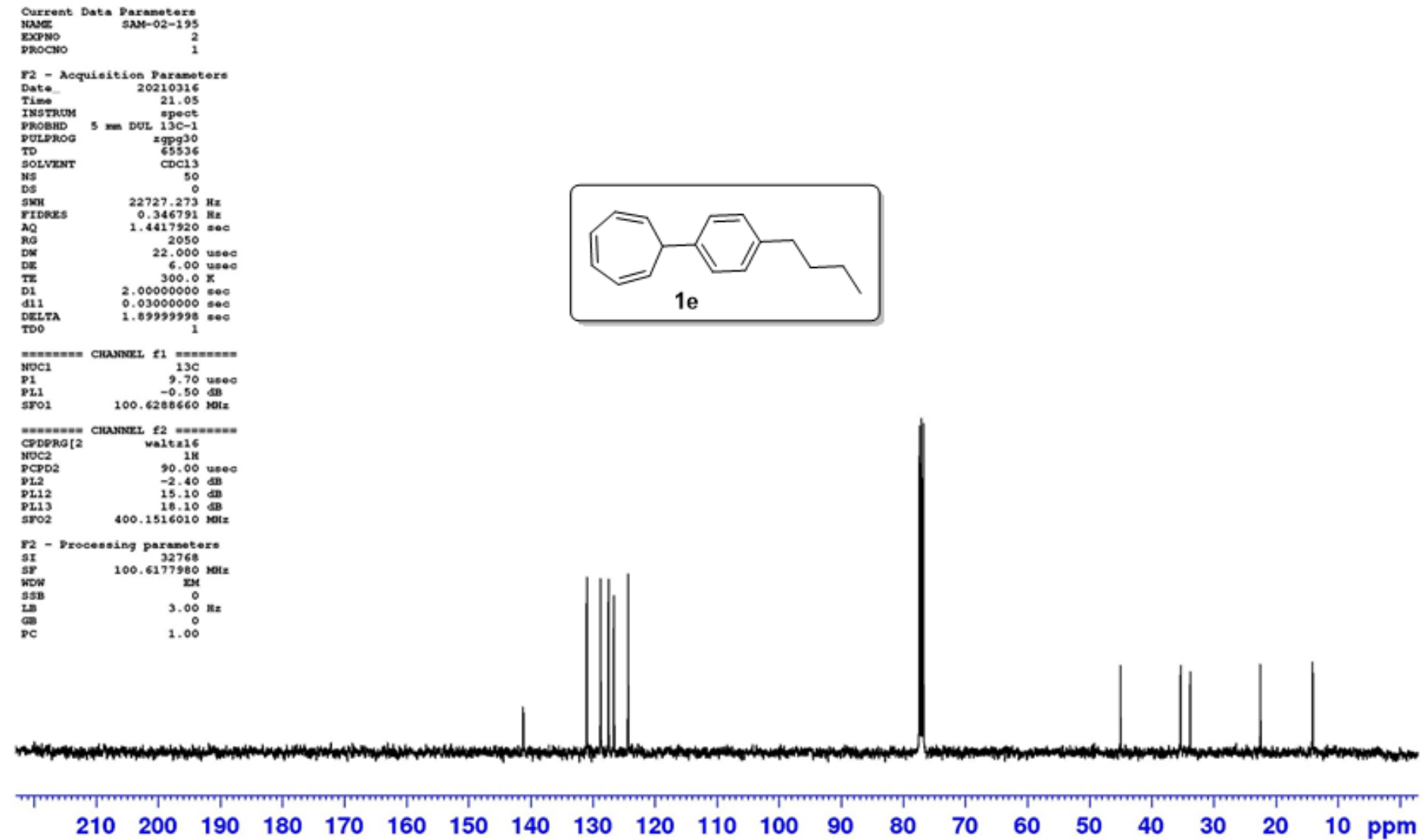
Solvent: $\mathrm{CDCl}_{3}$

SFO1: $400 \mathrm{MHz}$

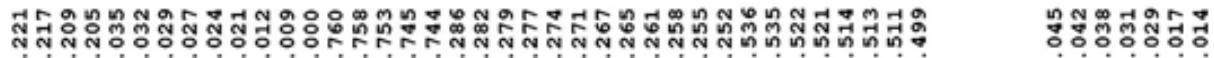

0606060060 on

(1)

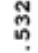

$\longrightarrow$

SAM-02-199
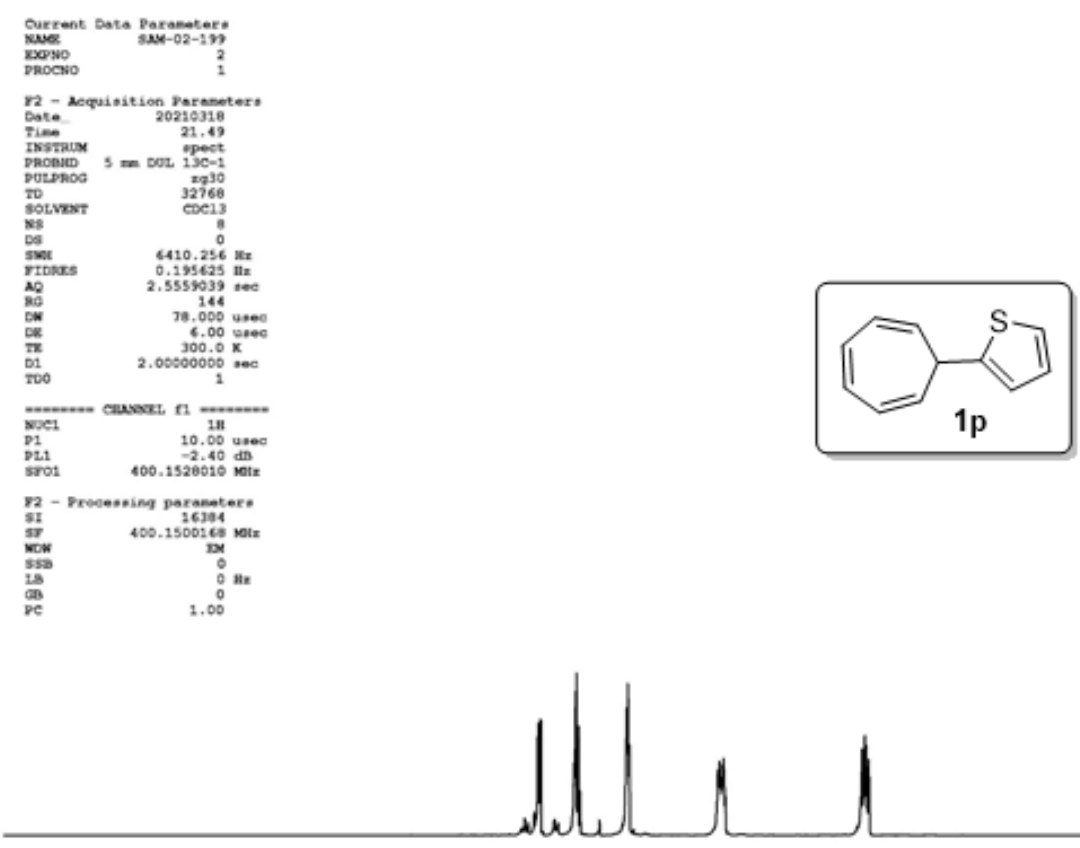

$9.5 \quad 9.0$

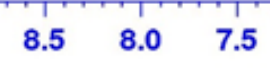

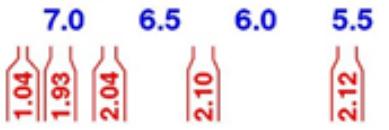

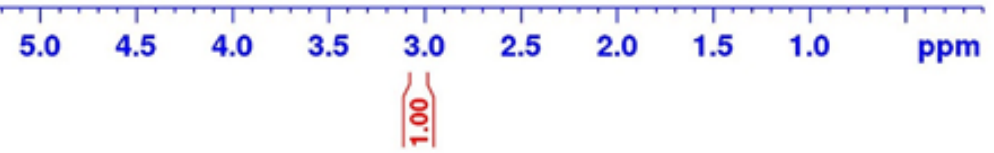


Solvent: $\mathrm{CDCl}_{3}$

SFO1: $100 \mathrm{MHz}$

\section{SAM-02-199}
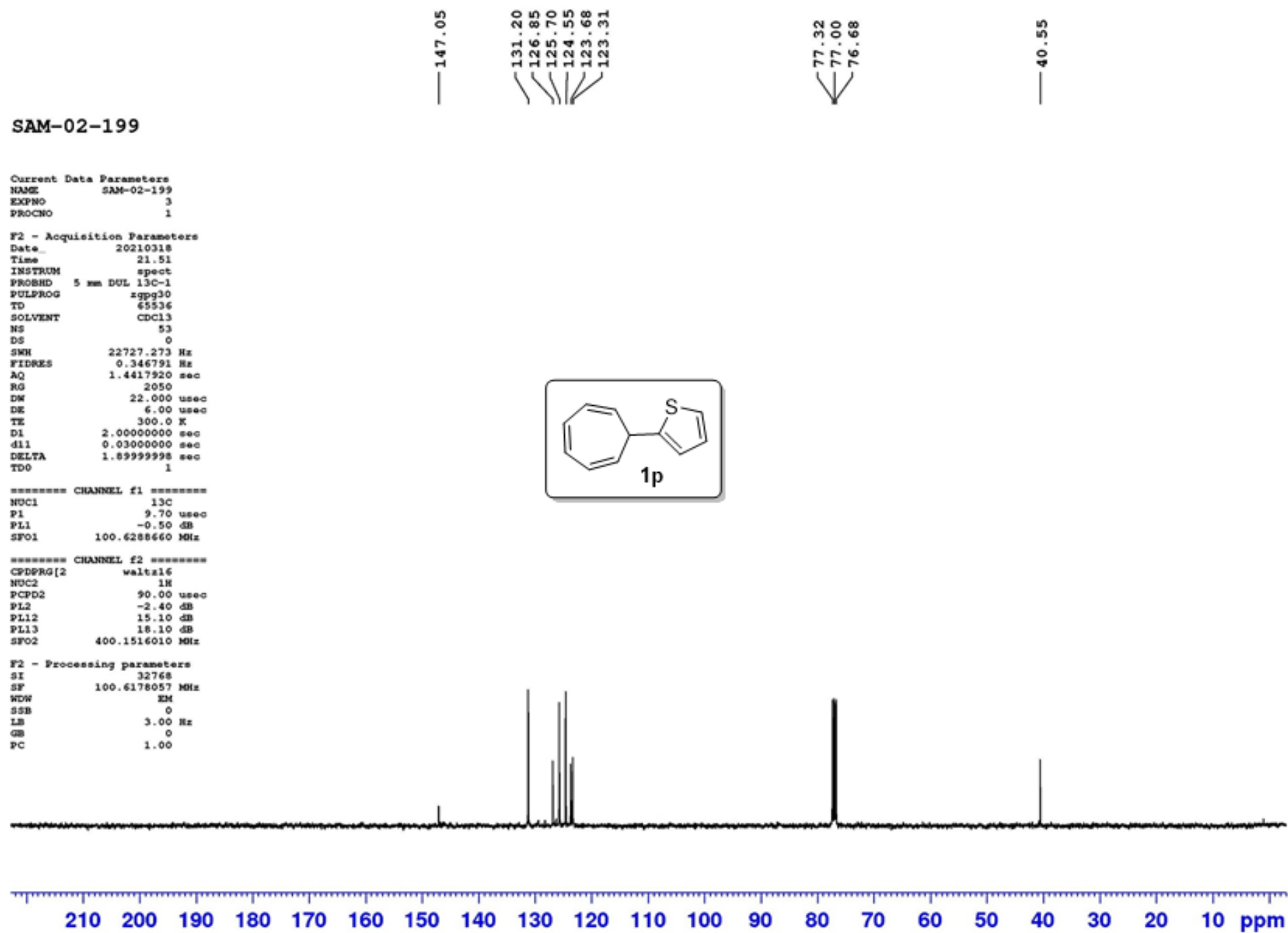
Solvent: $\mathrm{CDCl}_{3}$

SFO1: $400 \mathrm{MHz}$

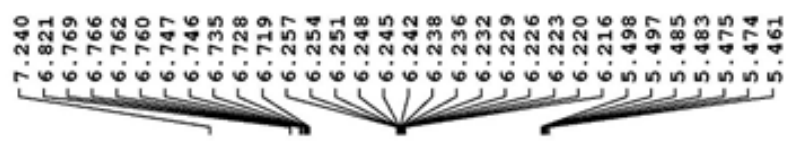

ฟ

SAM-02-208
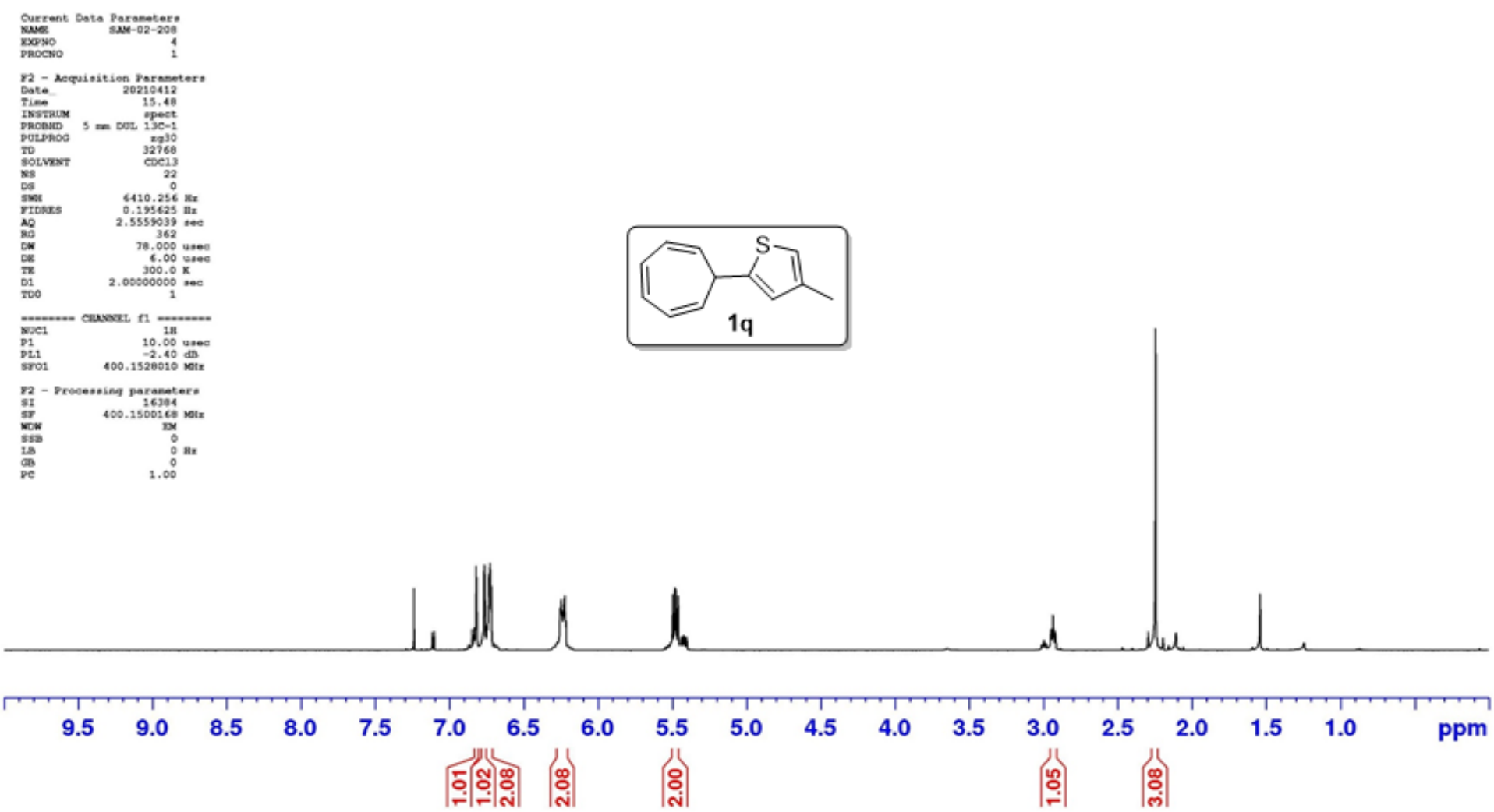
Solvent: $\mathrm{CDCl}_{3}$

SFO1: $100 \mathrm{MHz}$

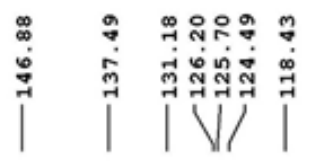

쏘요

FF⿱

$\bigvee$

$\stackrel{\circ}{\circ}$

$\stackrel{\circ}{\infty}$

|

SAM-02-208
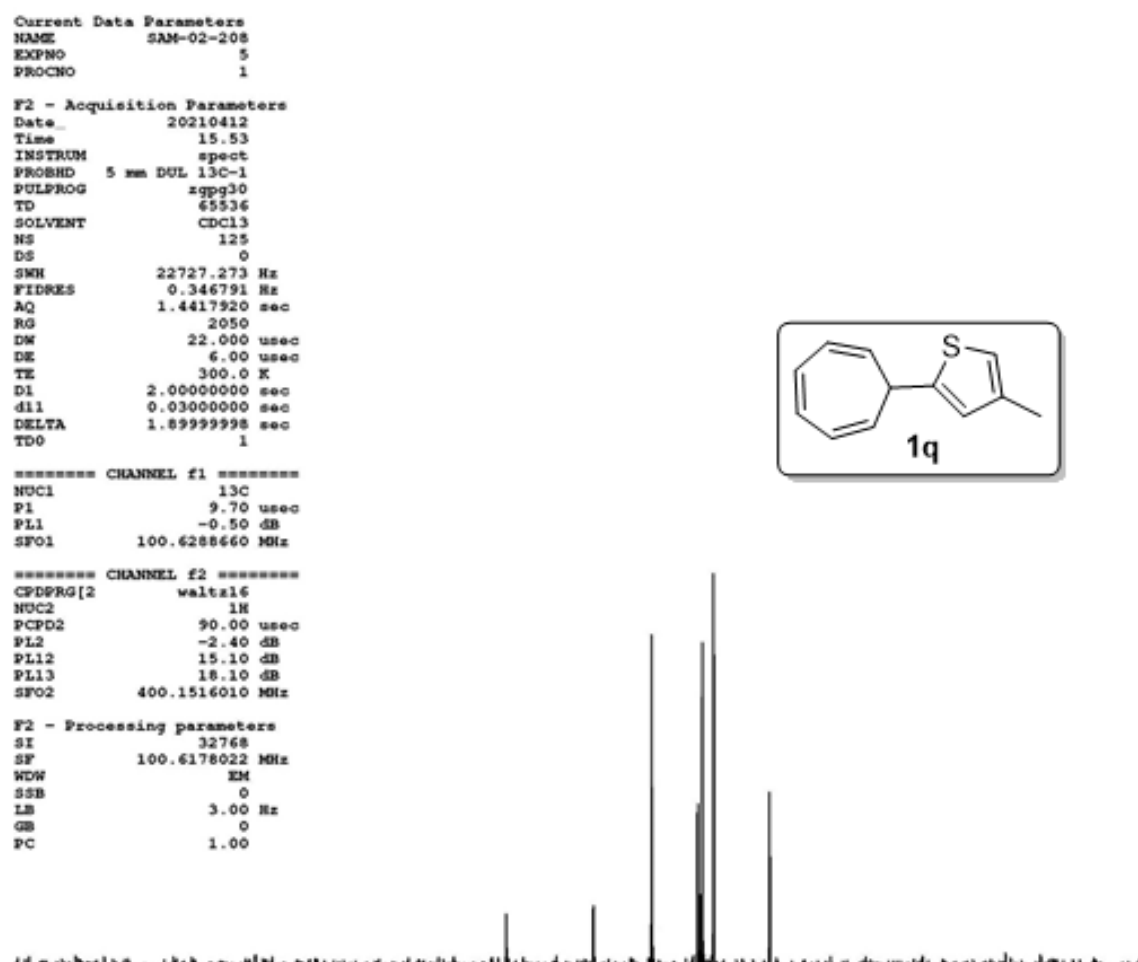

Shem

$\begin{array}{lllllllllllllllllll}190 & 180 & 170 & 160 & 150 & 140 & 130 & 120 & 110 & 100 & 90 & 80 & 70 & 60 & 50 & 40 & 30 & 20 & \mathrm{ppm}\end{array}$ 
Solvent: $\mathrm{CDCl}_{3}$

SFO1: $400 \mathrm{MHz}$

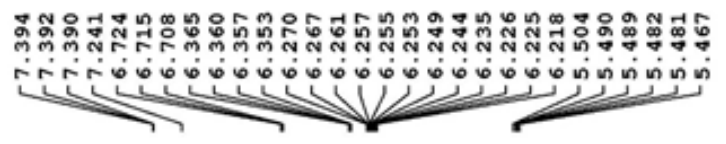

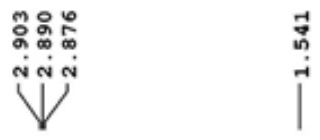

\section{SAM-02-203}

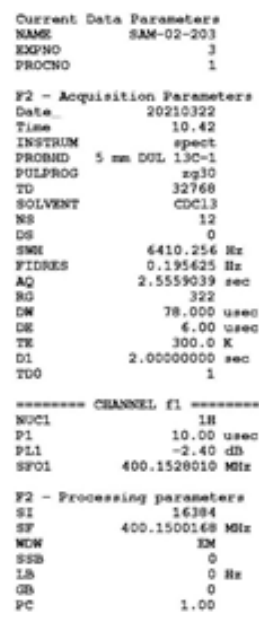
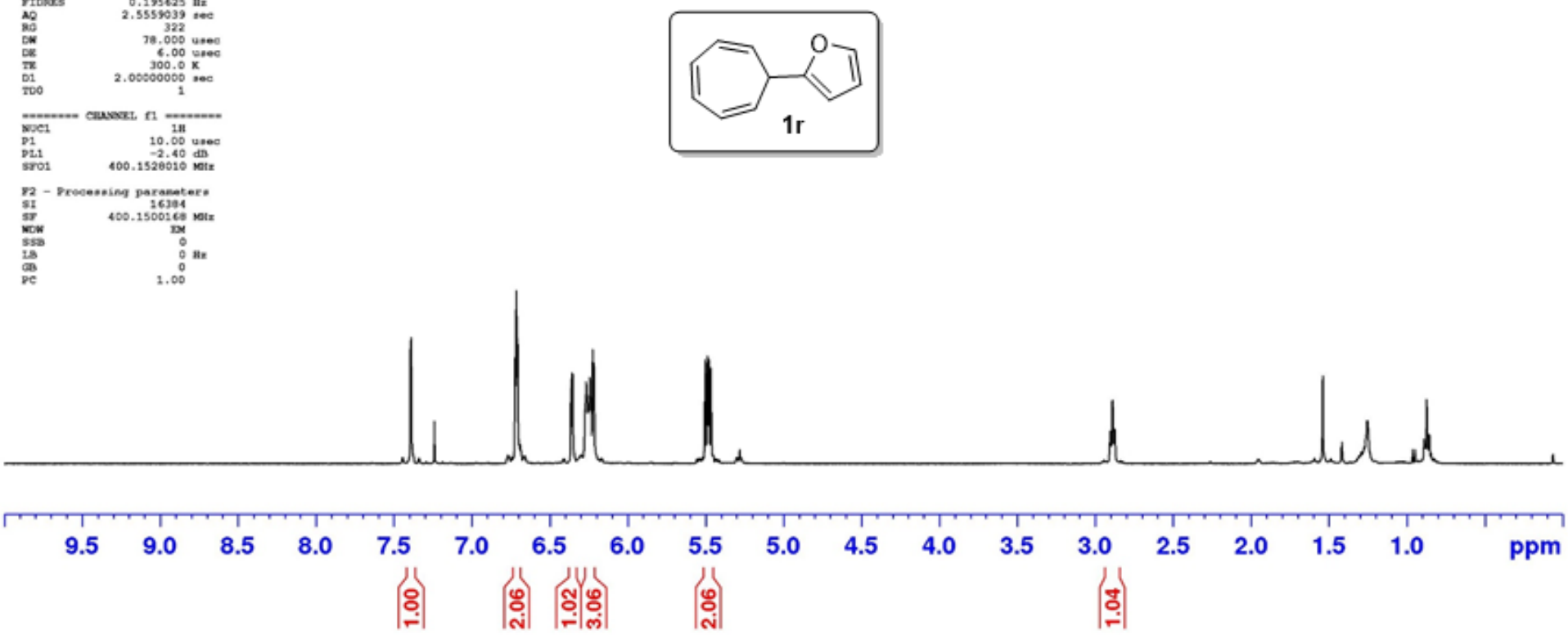
Solvent: $\mathrm{CDCl}_{3}$

SFO1: $100 \mathrm{MHz}$
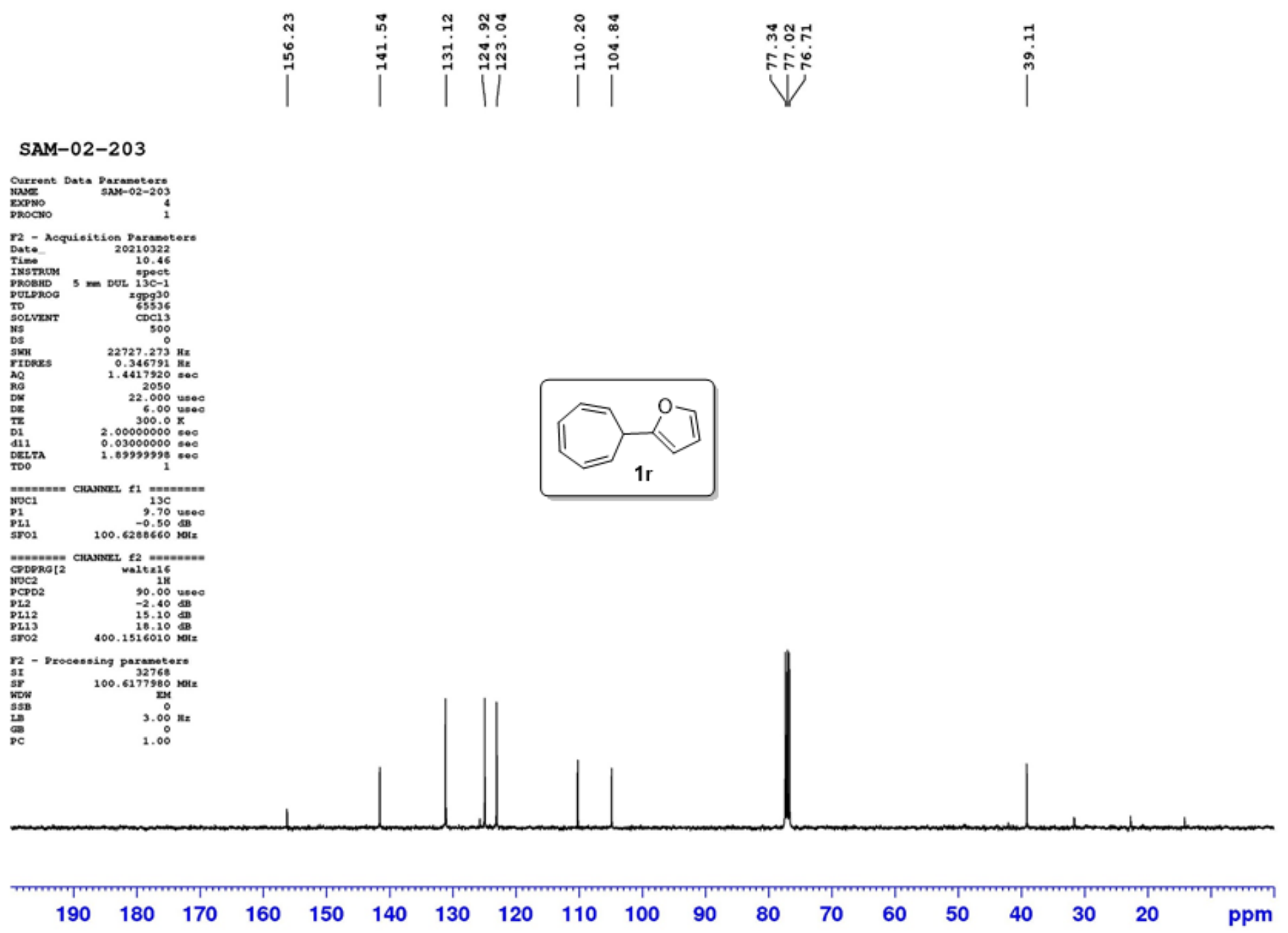
Solvent: $\mathrm{CDCl}_{3}$ SFO1: $700 \mathrm{MHz}$

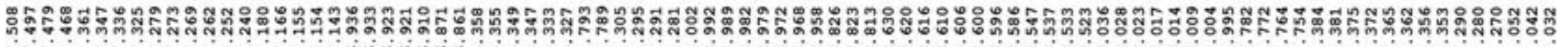

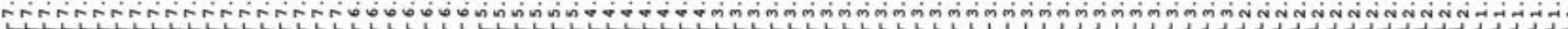

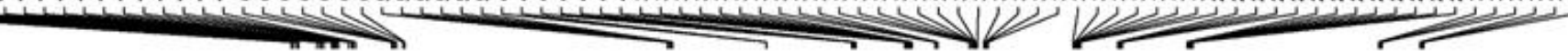

\section{SAM-02-106-D-1}

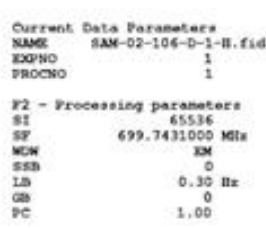
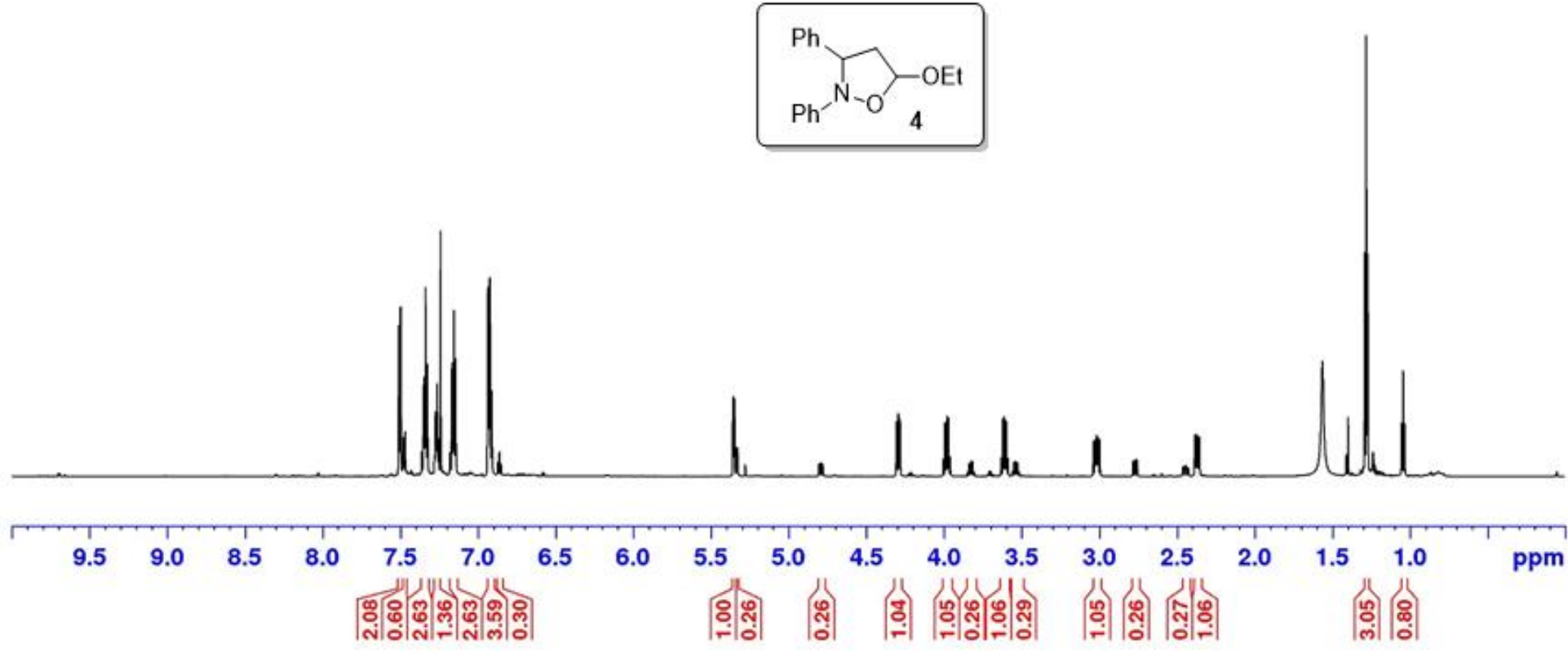
Solvent: $\mathrm{CDCl}_{3}$

SFO1: $175 \mathrm{MHz}$

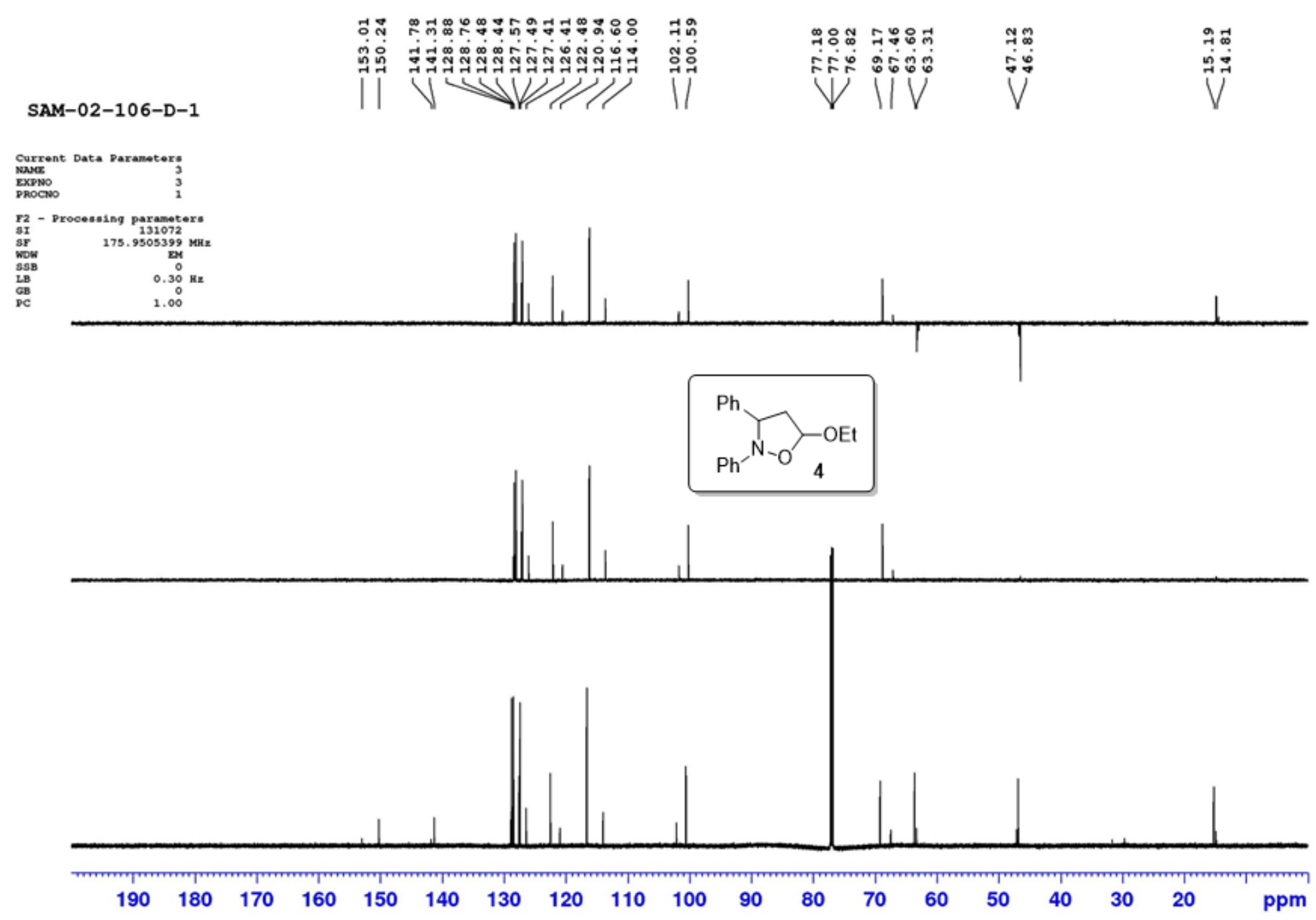


Solvent: $\mathrm{CDCl}_{3}$

SFO1: $700 \mathrm{MHz}$

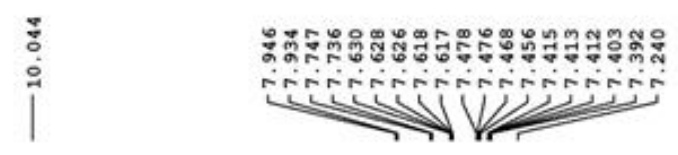

SAM-02-166-2

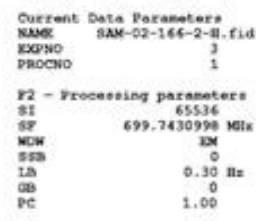

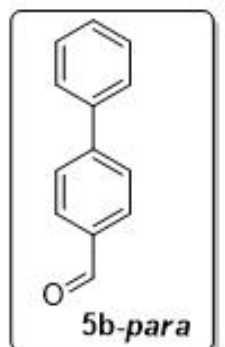

5b-para

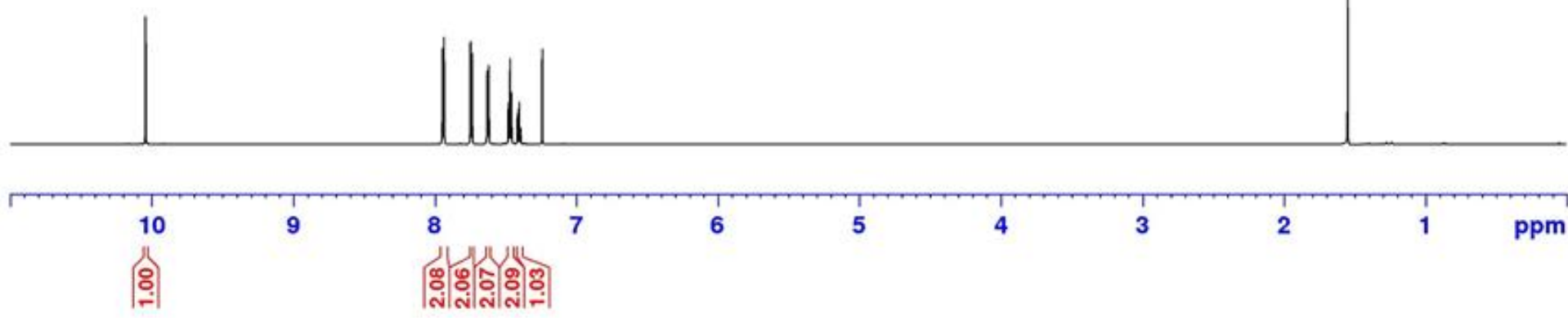


Solvent: $\mathrm{CDCl}_{3}$

SFO1: $175 \mathrm{MHz}$

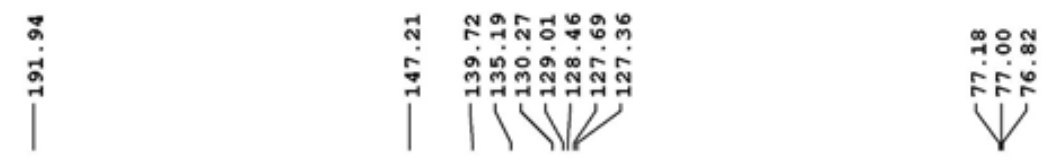

SAM-02-166-2
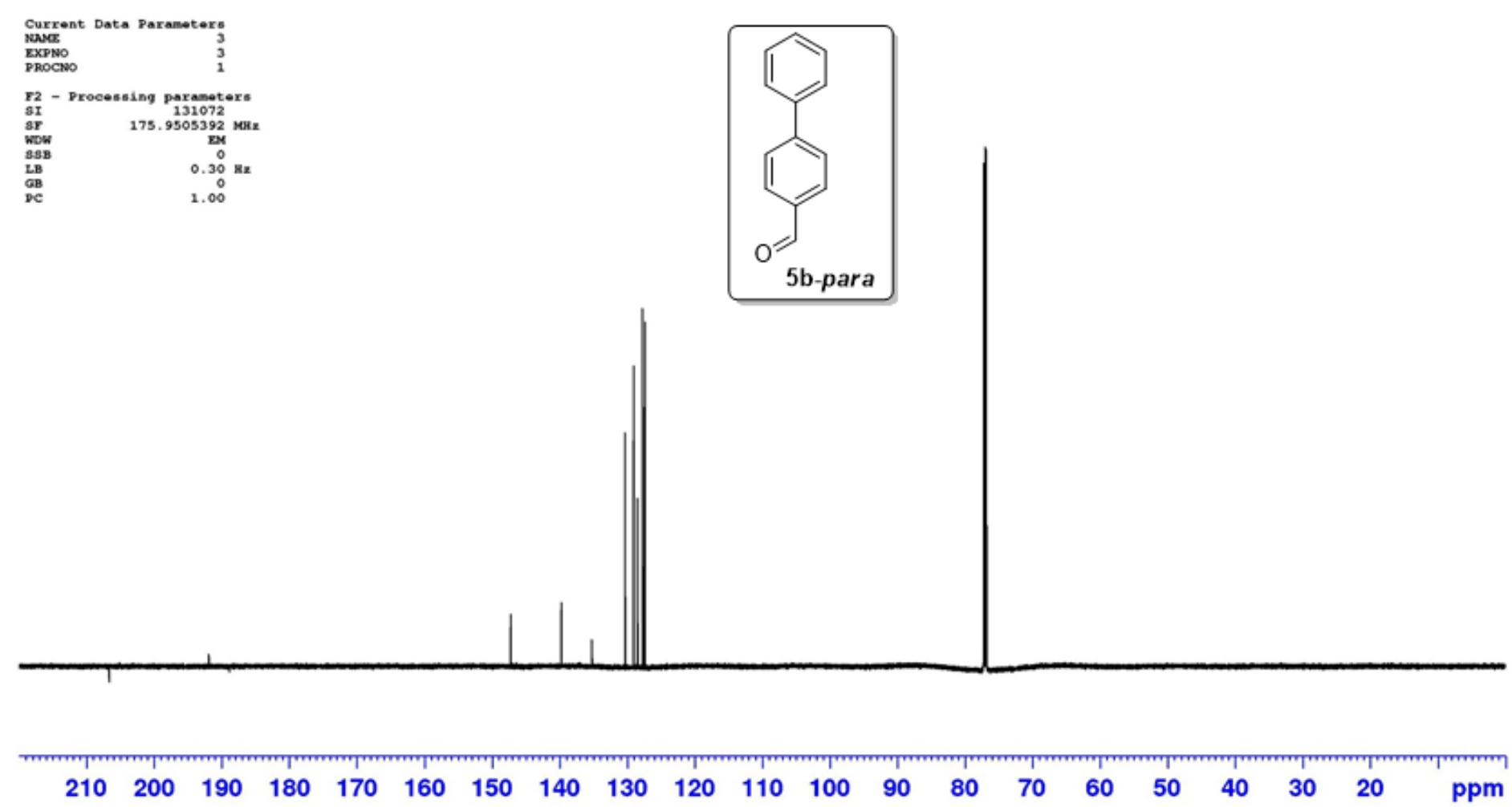
Solvent: $\mathrm{CDCl}_{3}$

SFO1: $700 \mathrm{MHz}$

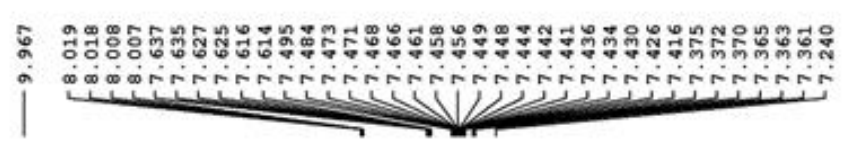

SAM-02-166-1

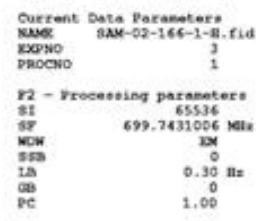

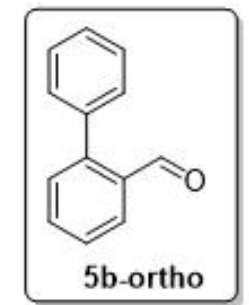

5b-ortho

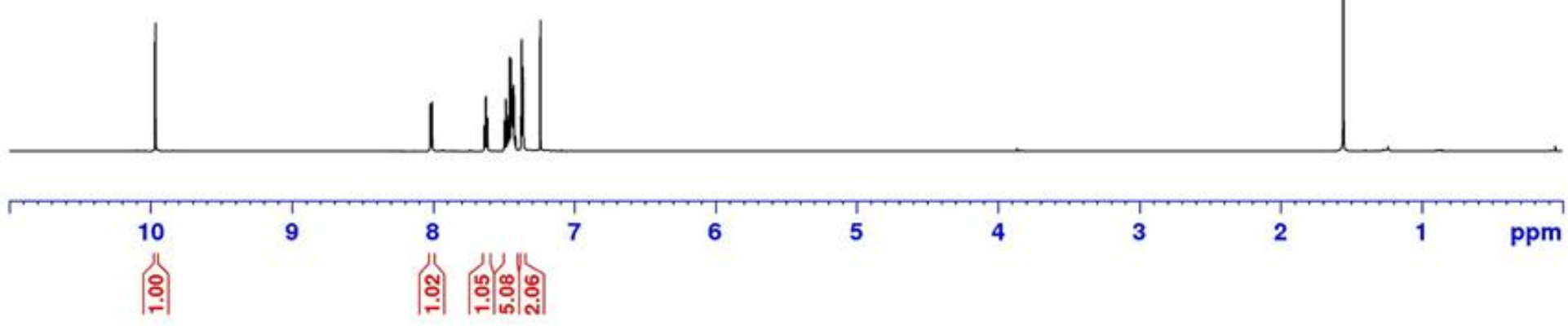


Solvent: $\mathrm{CDCl}_{3}$

SFO1: $175 \mathrm{MHz}$
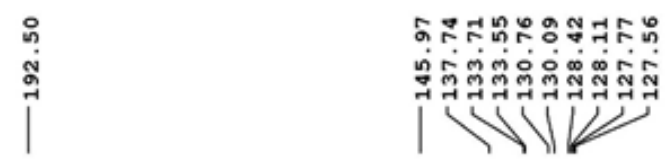

๓๐ำ

FF

SAM-02-166-1

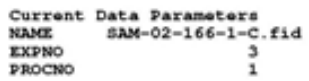

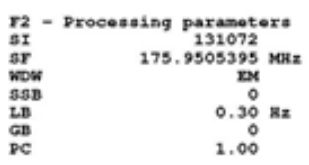
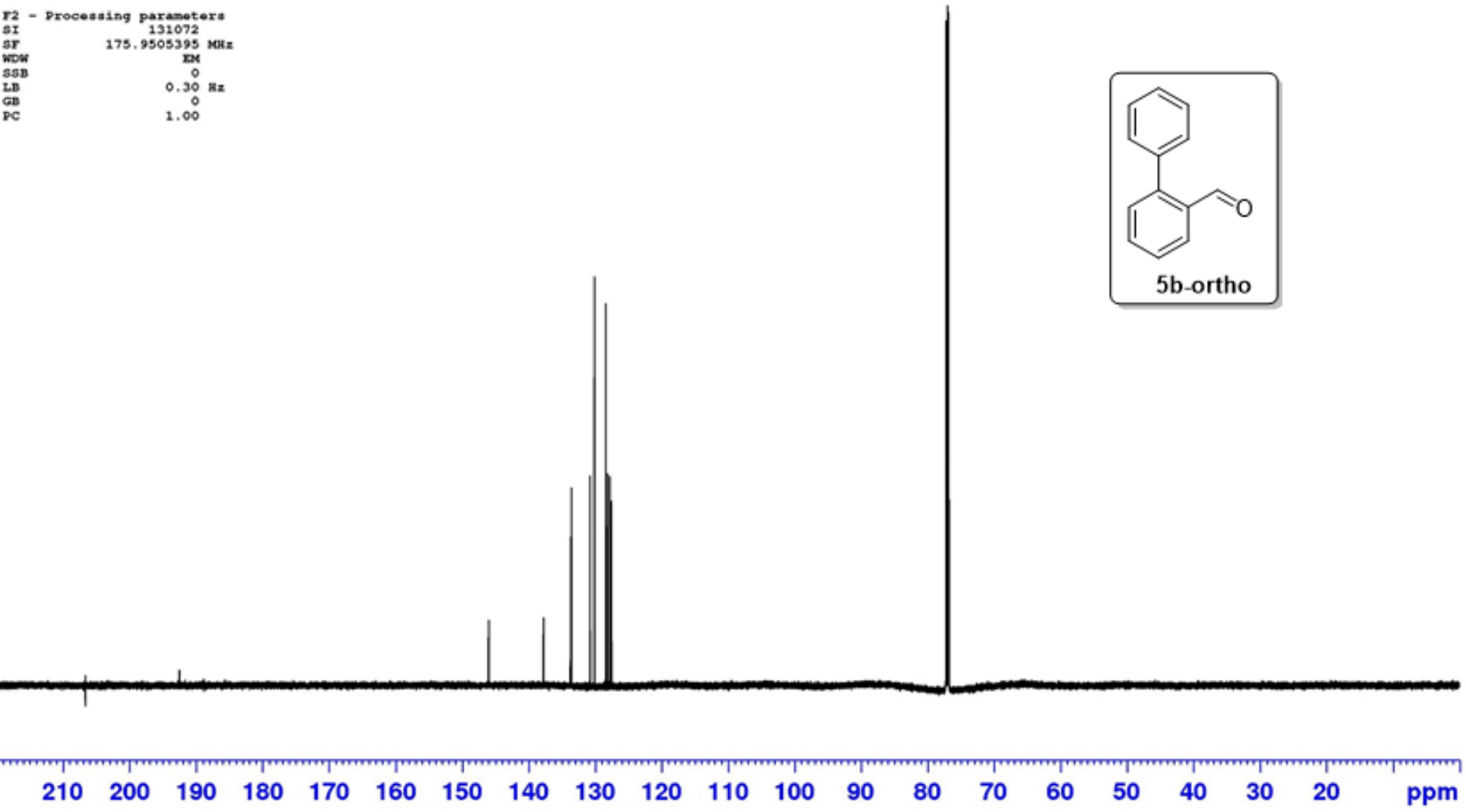
Solvent: $\mathrm{CDCl}_{3}$ SFO1: $700 \mathrm{MHz}$

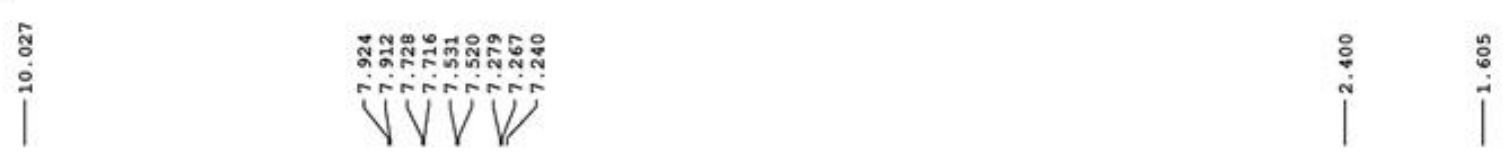

\section{SAM-02-177-2}
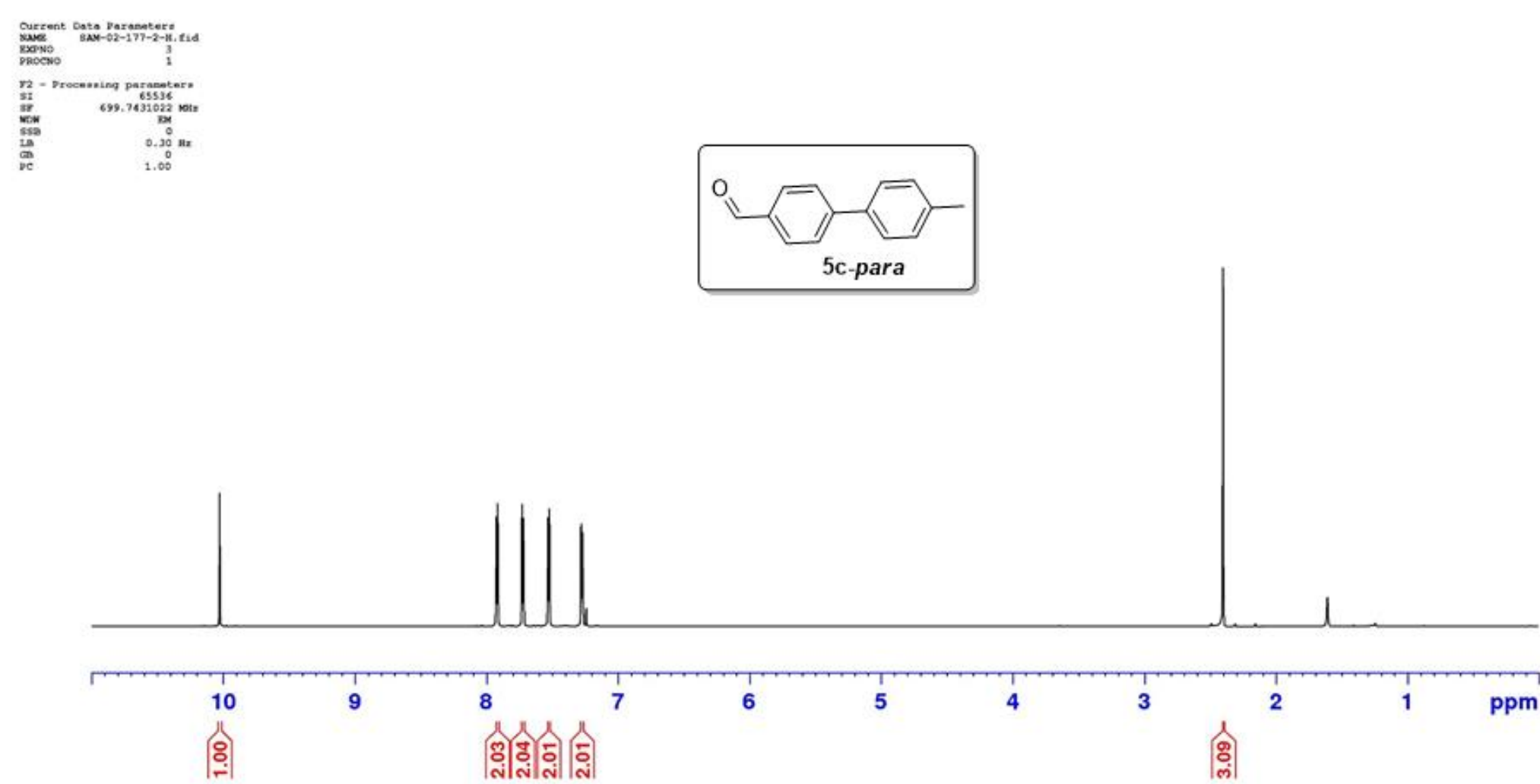
Solvent: $\mathrm{CDCl}_{3}$

SFO1: $175 \mathrm{MHz}$

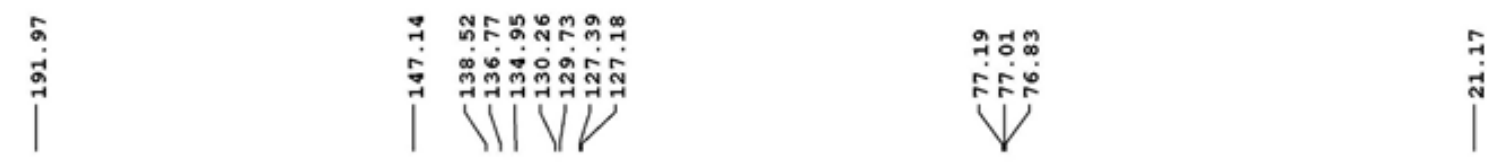

SAM-02-177-2
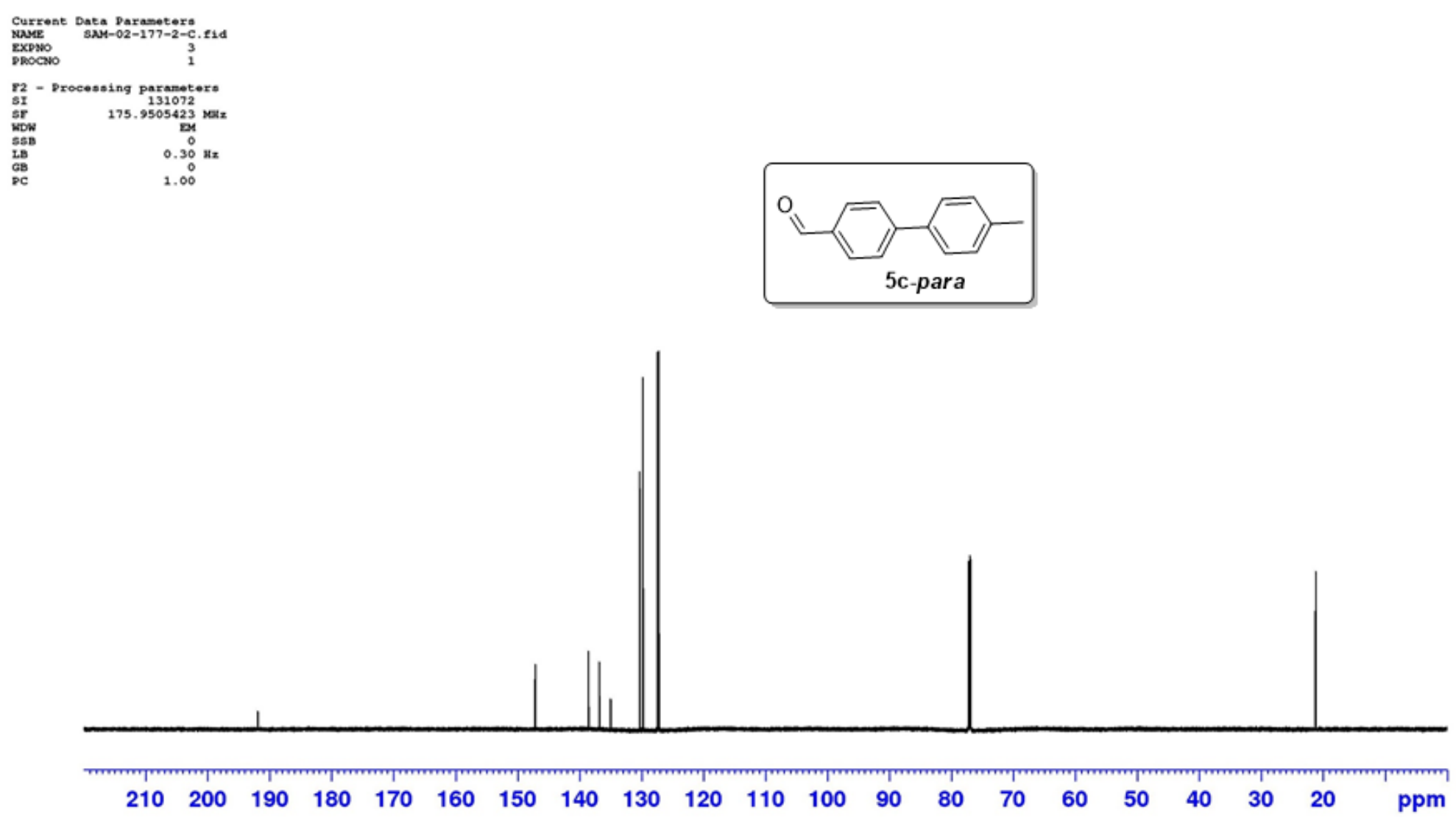
Solvent: $\mathrm{CDCl}_{3}$

SFO1: $700 \mathrm{MHz}$

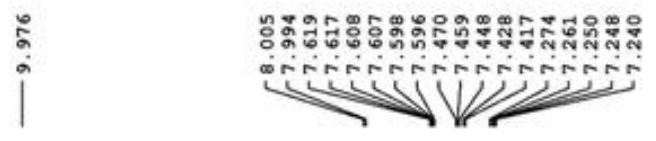

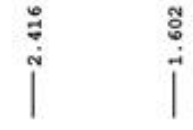

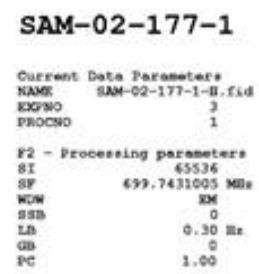

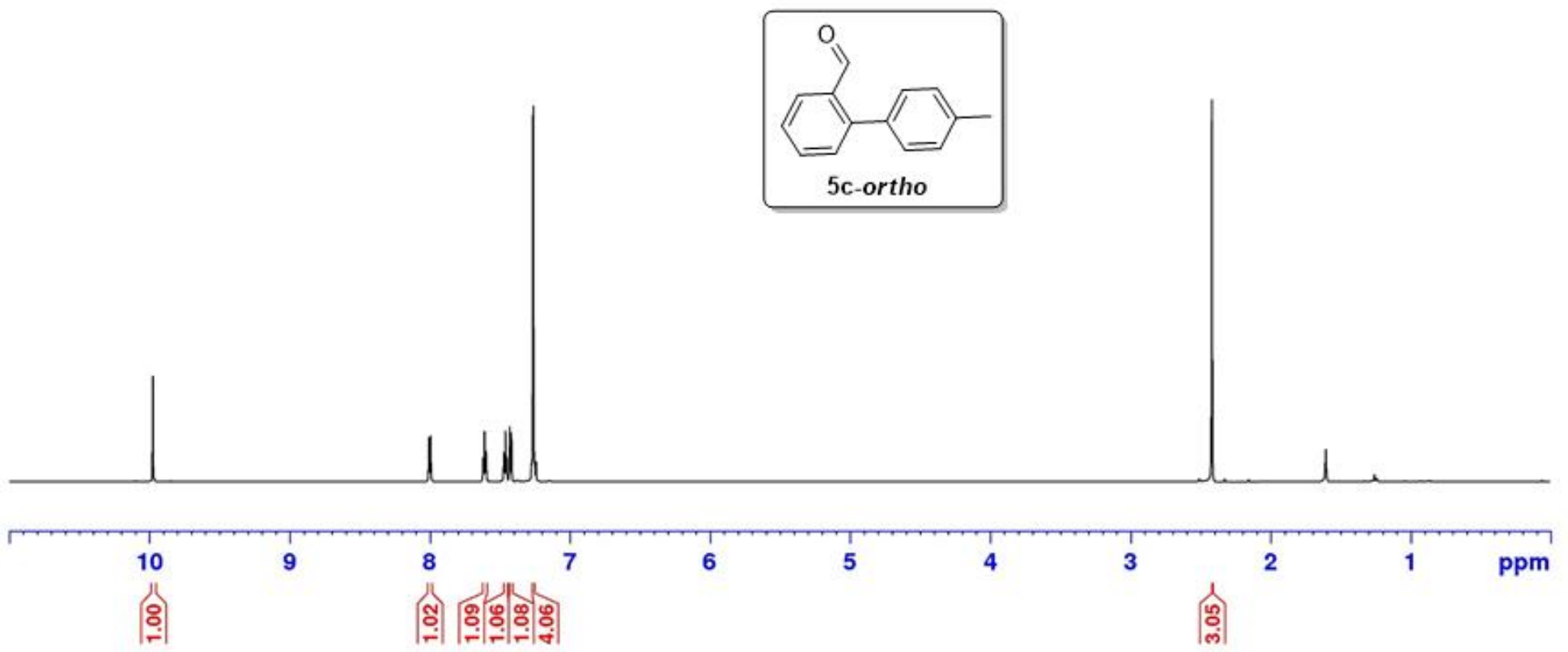


Solvent: $\mathrm{CDCl}_{3}$

SFO1: $175 \mathrm{MHz}$

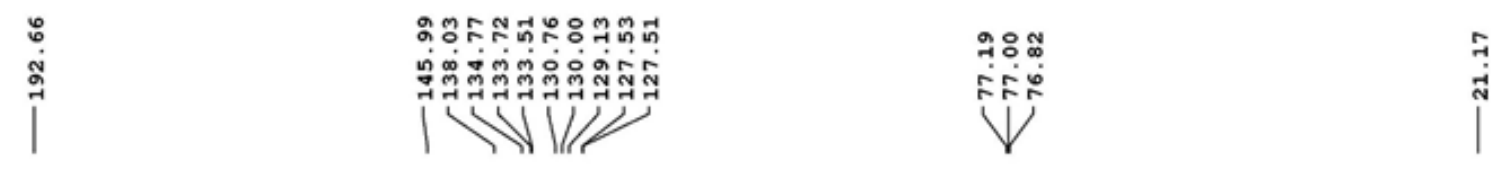

SAM-02-177-1
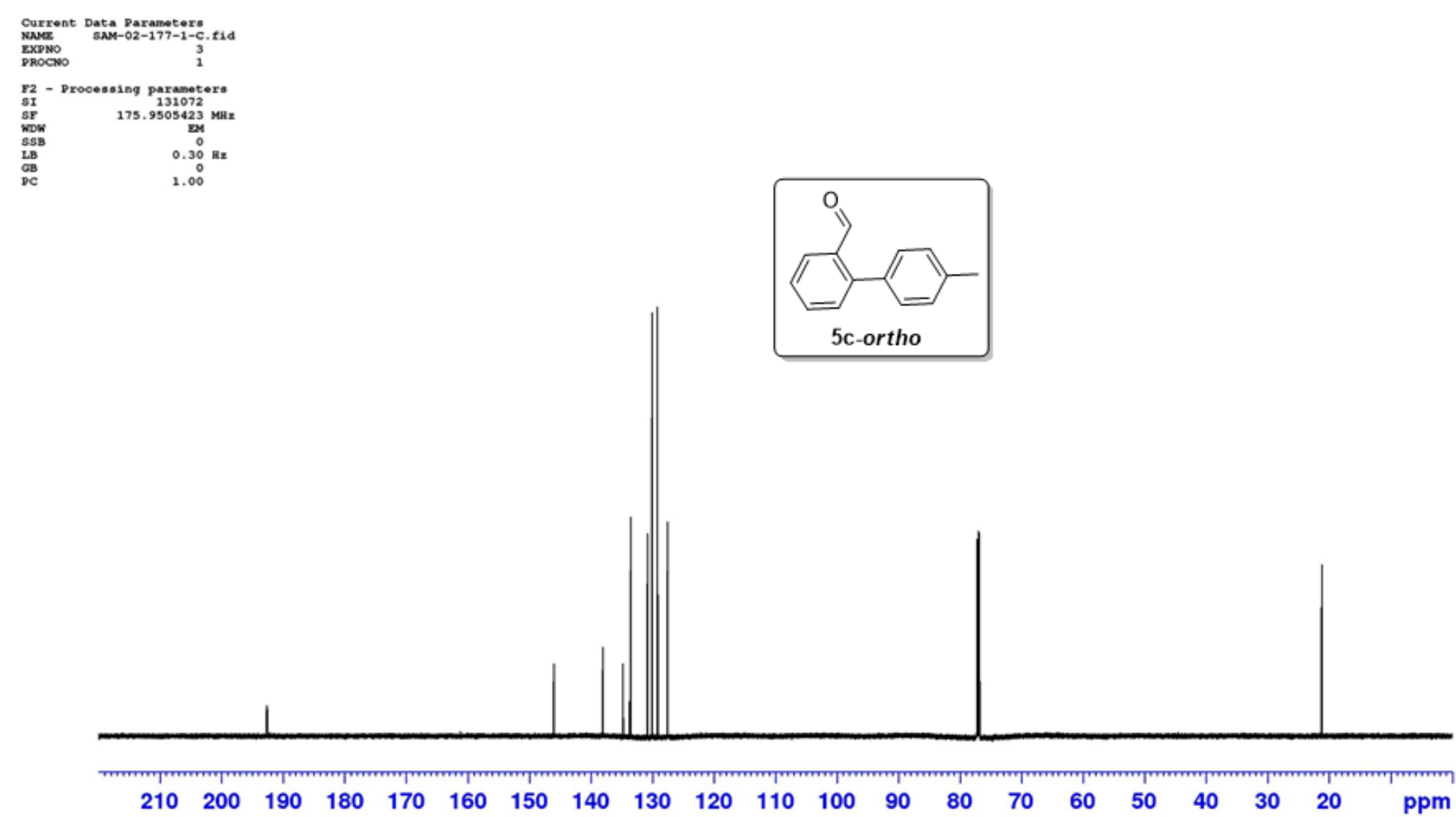
Solvent: $\mathrm{CDCl}_{3}$ SFO1: $700 \mathrm{MHz}$

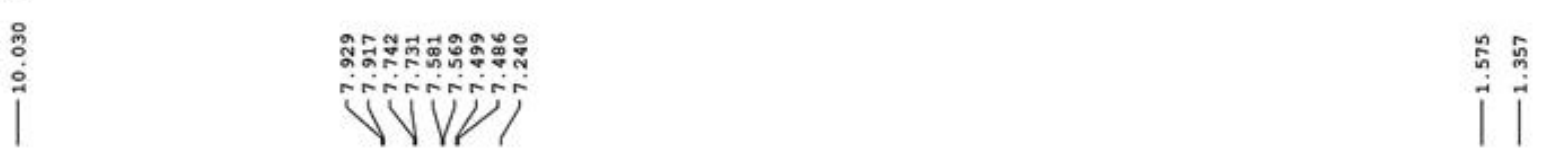

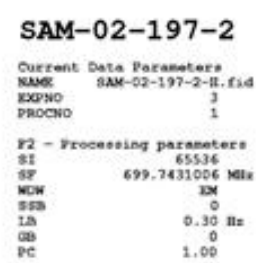
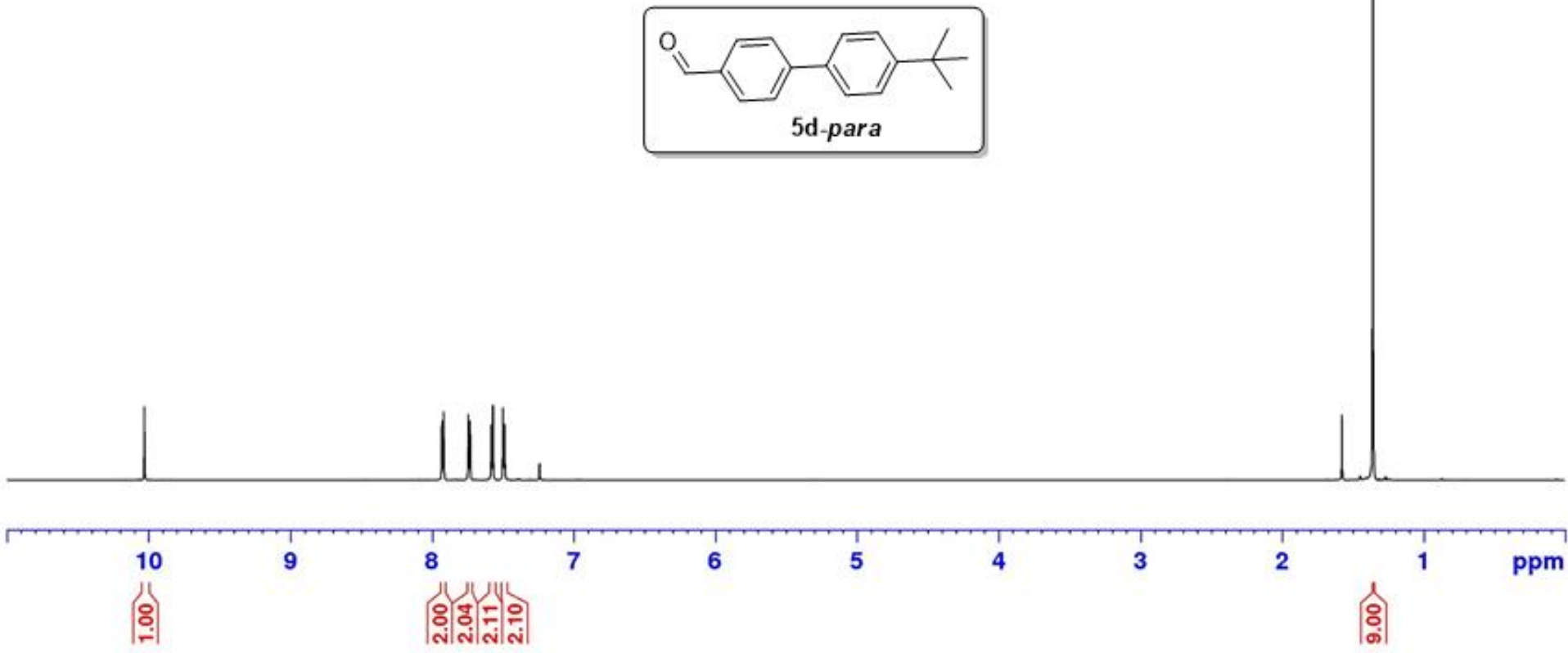
Solvent: $\mathrm{CDCl}_{3}$

SFO1: $175 \mathrm{MHz}$

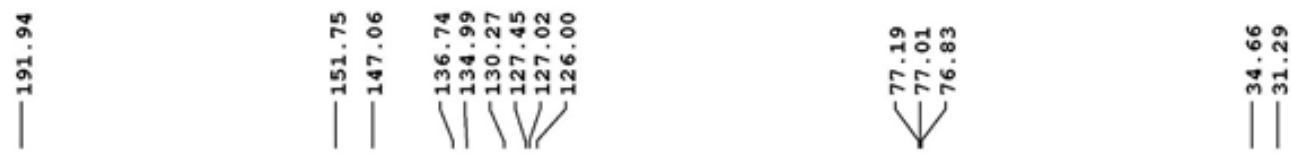

\section{SAM-02-197-2}
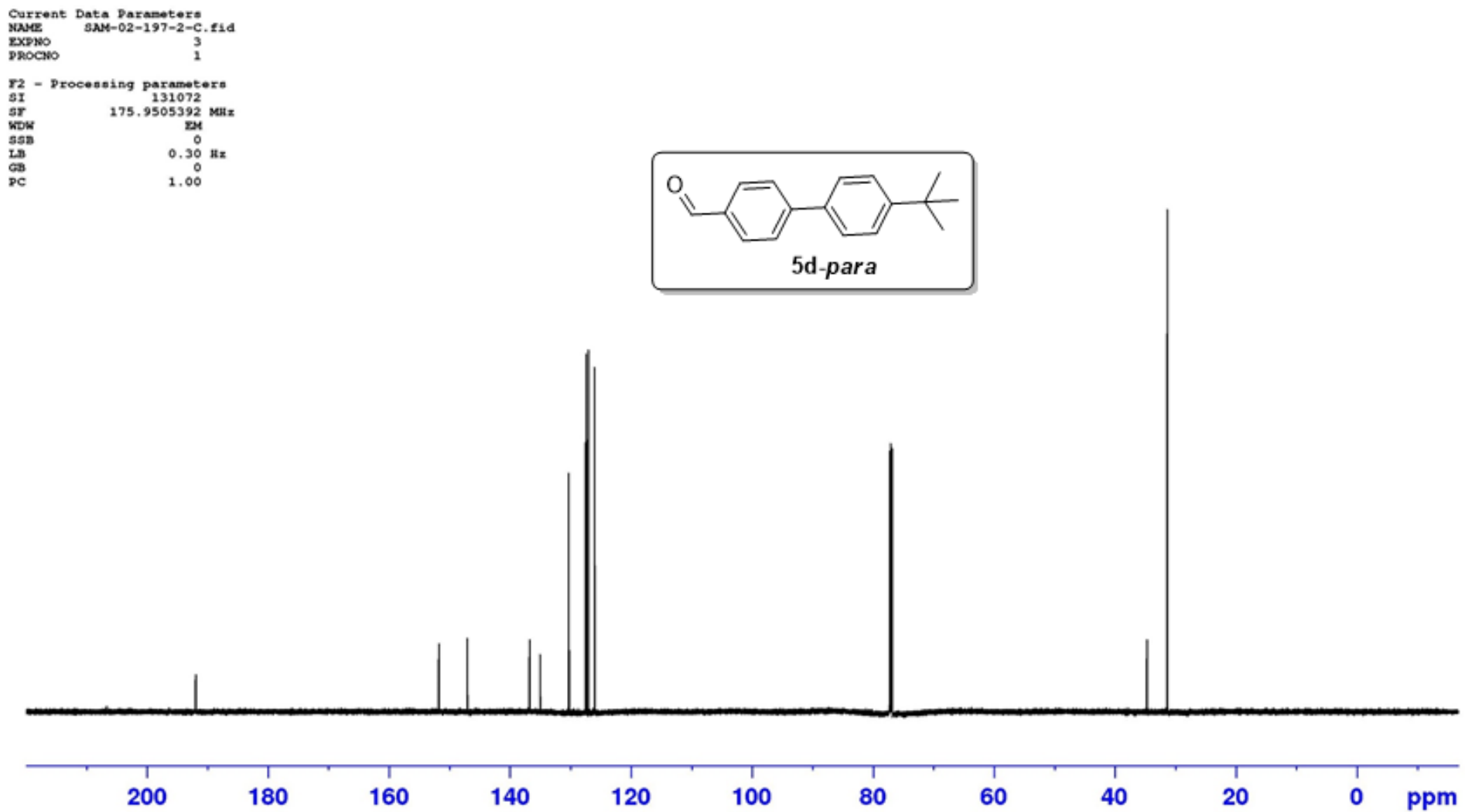
Solvent: $\mathrm{CDCl}_{3}$

SFO1: $400 \mathrm{MHz}$

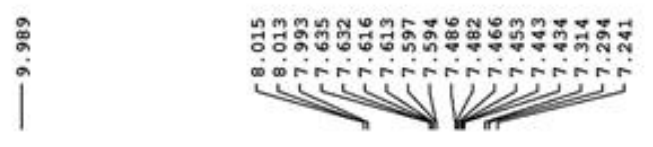

SAM-02-197-1-H
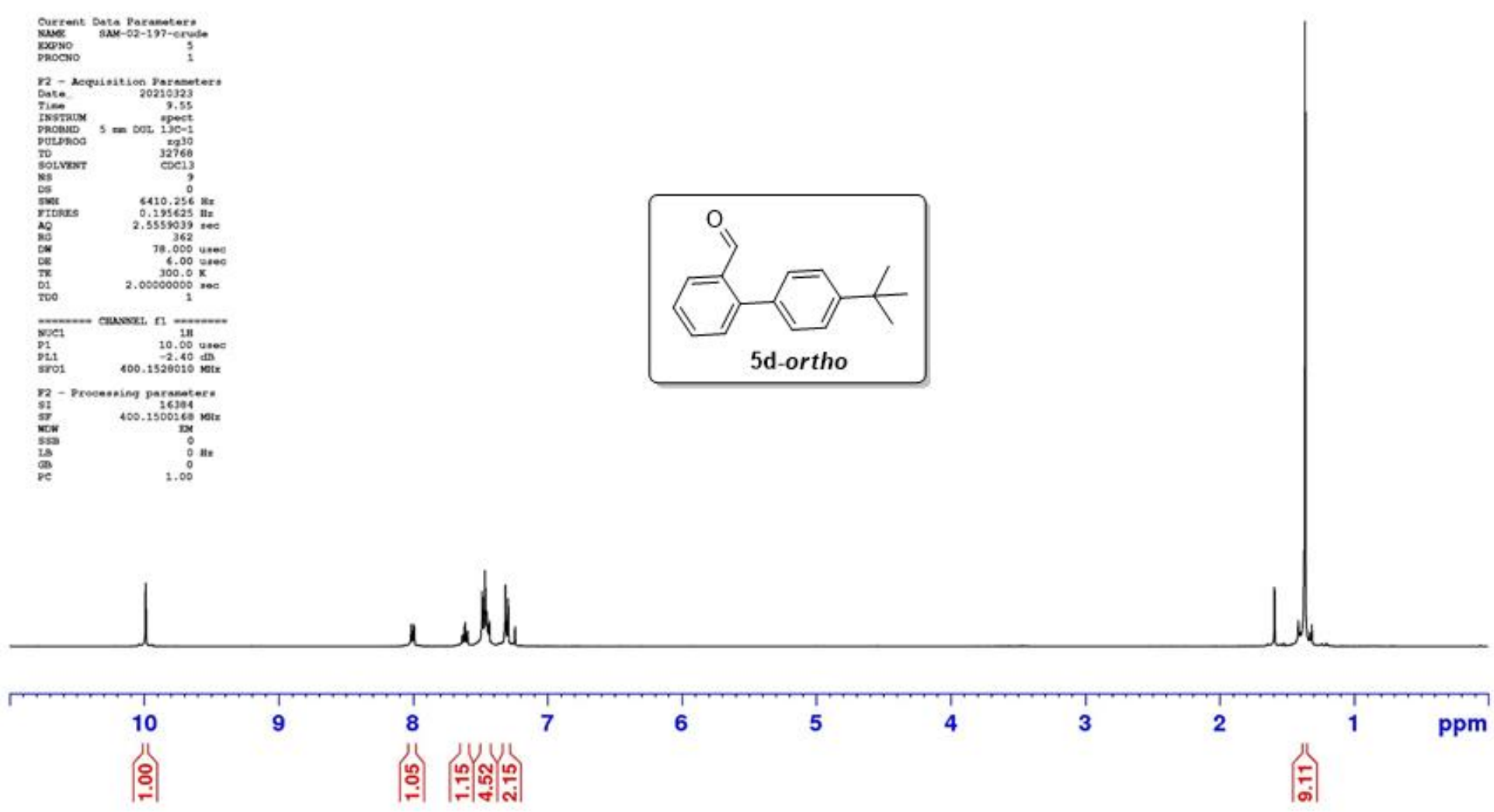
Solvent: $\mathrm{CDCl}_{3}$

SFO1: $175 \mathrm{MHz}$

|

SAM-02-197-1
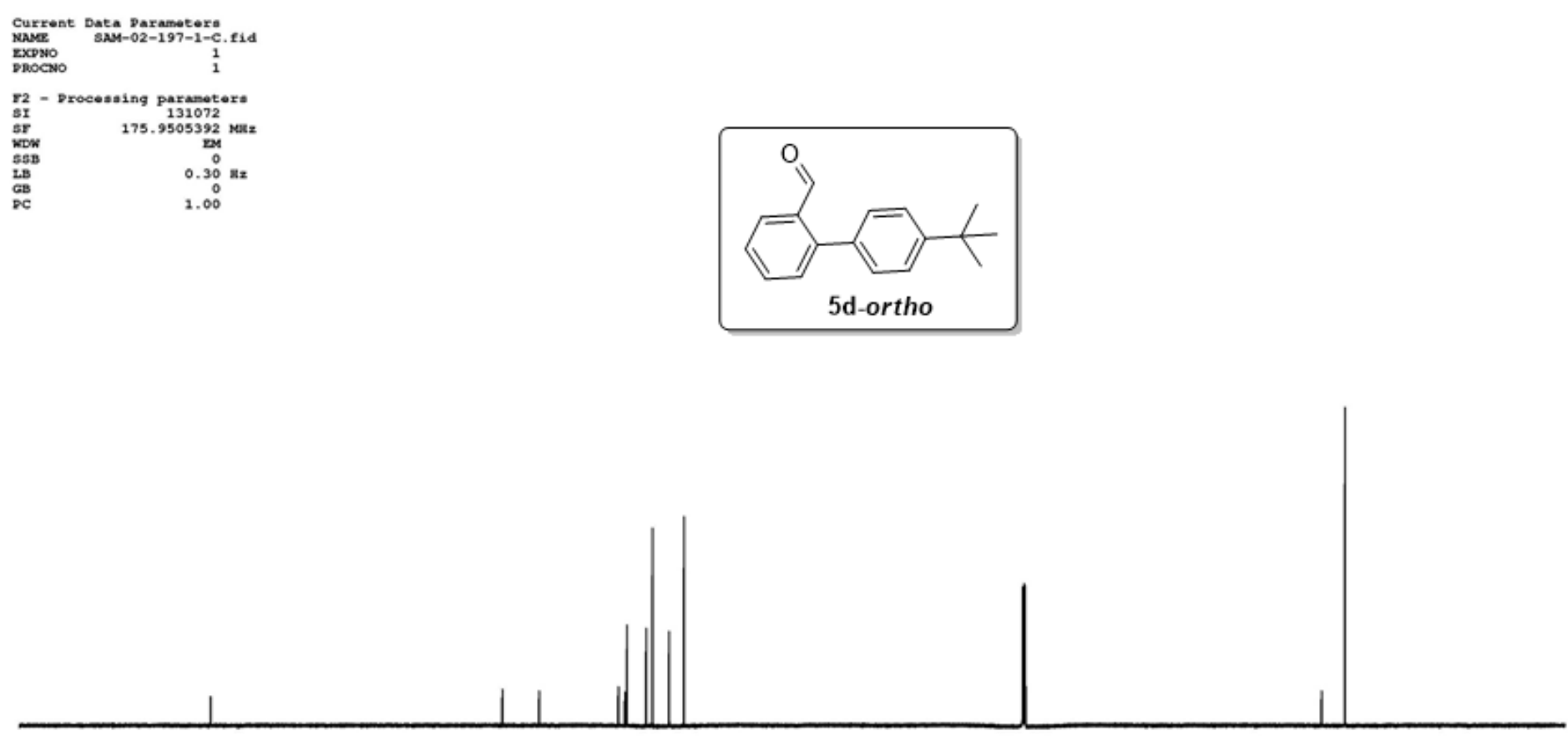

$$
\begin{array}{llllllllllllllllllllll}
210 & 200 & 190 & 180 & 170 & 160 & 150 & 140 & 130 & 120 & 110 & 100 & 90 & 80 & 70 & 60 & 50 & 40 & 30 & 20 & \mathrm{ppm}
\end{array}
$$


Solvent: $\mathrm{CDCl}_{3}$

SFO1: $700 \mathrm{MHz}$
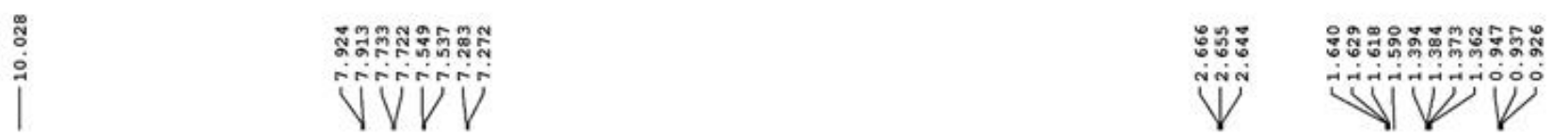

SAM-02-198-2

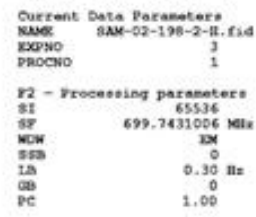
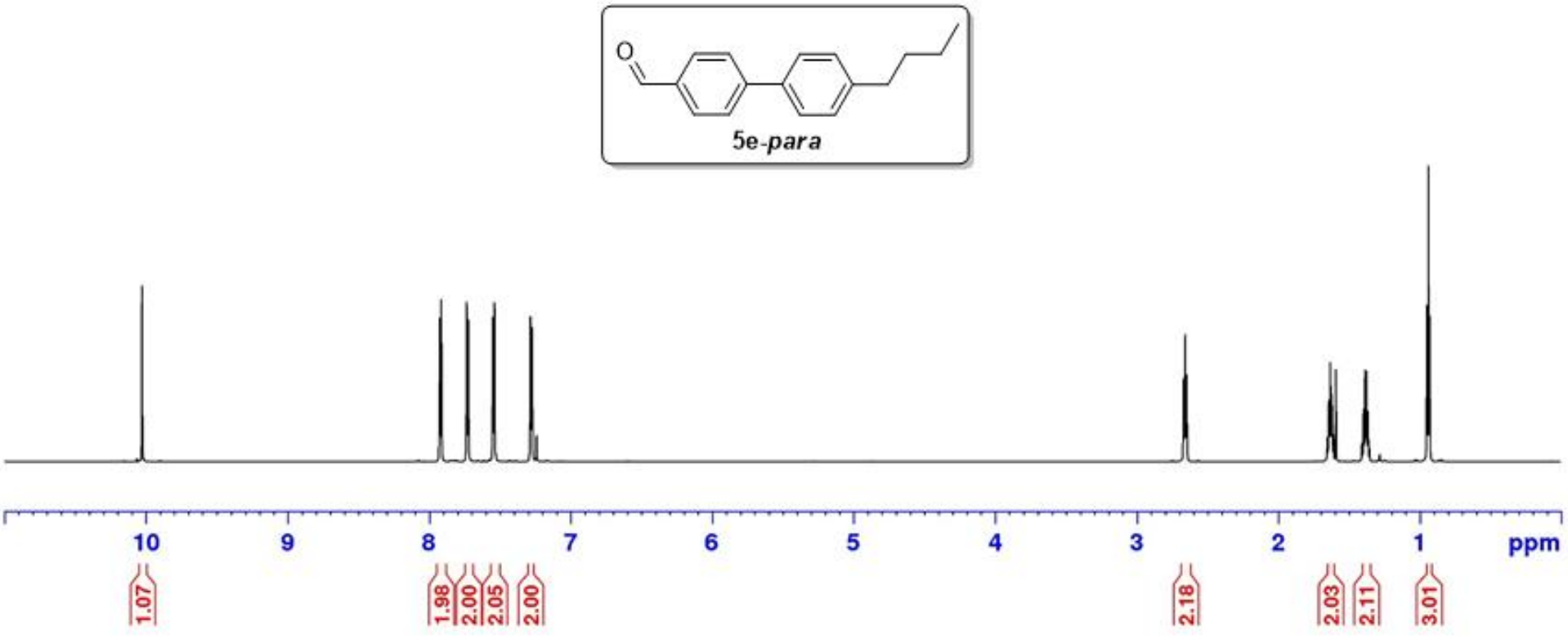
Solvent: $\mathrm{CDCl}_{3}$

SFO1: $175 \mathrm{MHz}$

|

\section{SAM-02-198-2}
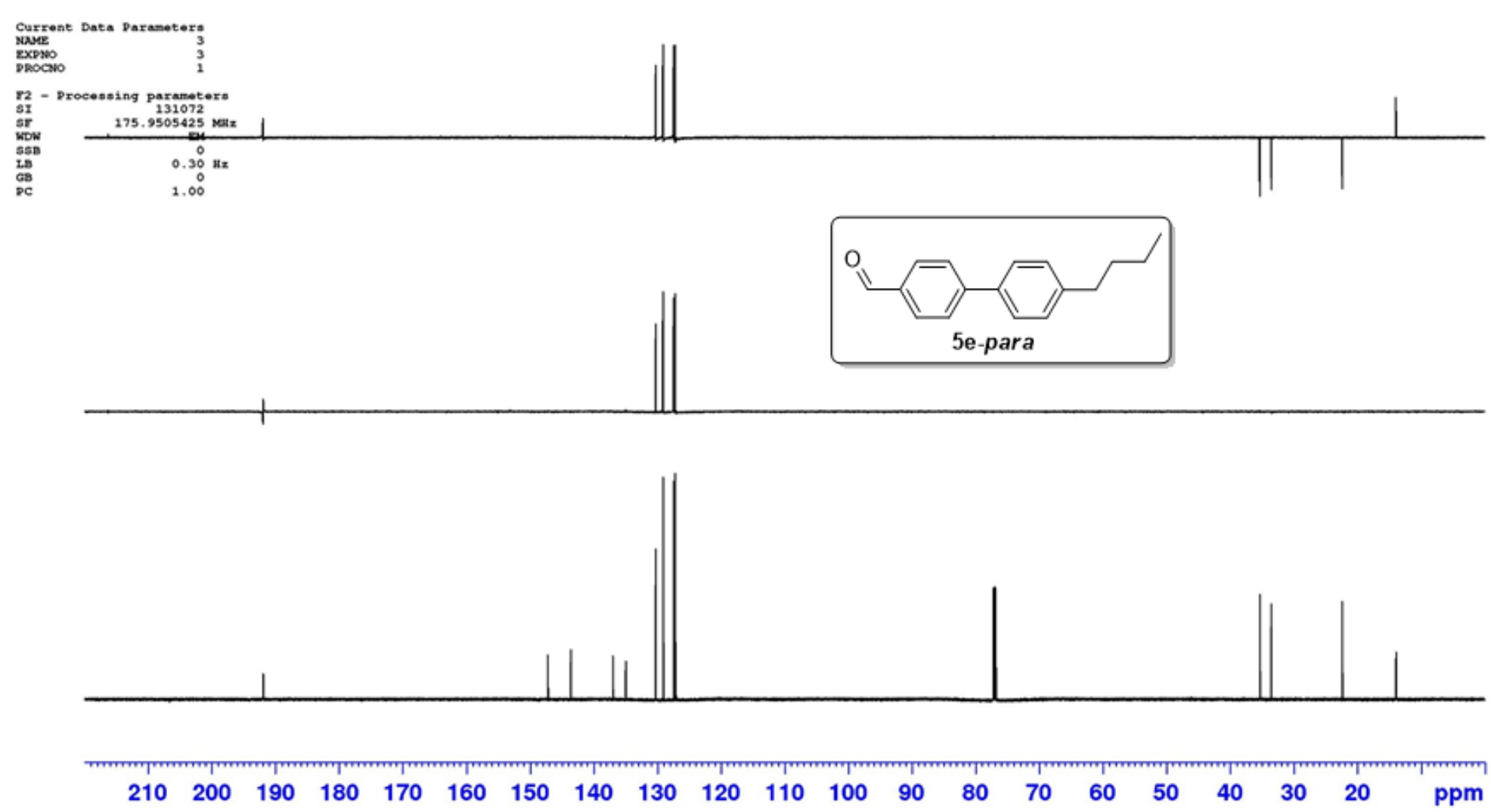
Solvent: $\mathrm{CDCl}_{3}$

SFO1: $700 \mathrm{MHz}$
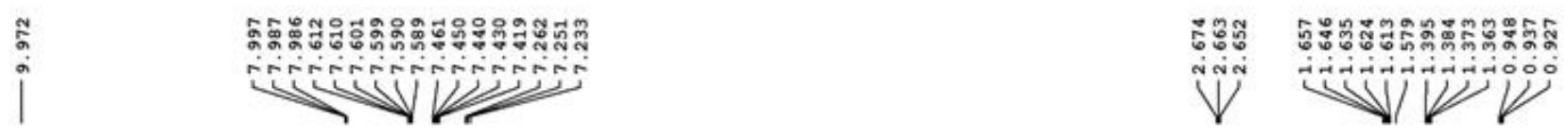

SAM-02-198-1

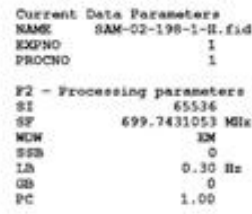

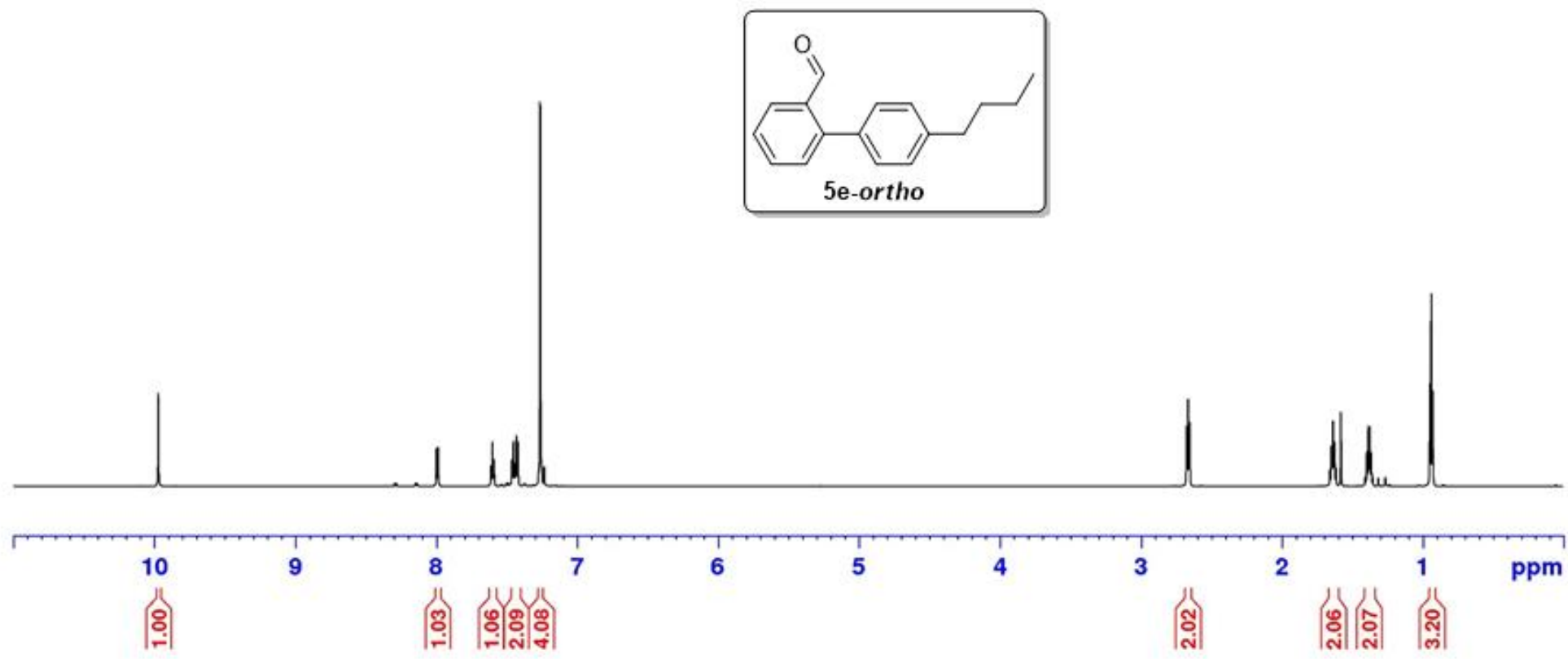


Solvent: $\mathrm{CDCl}_{3}$

SFO1: $175 \mathrm{MHz}$
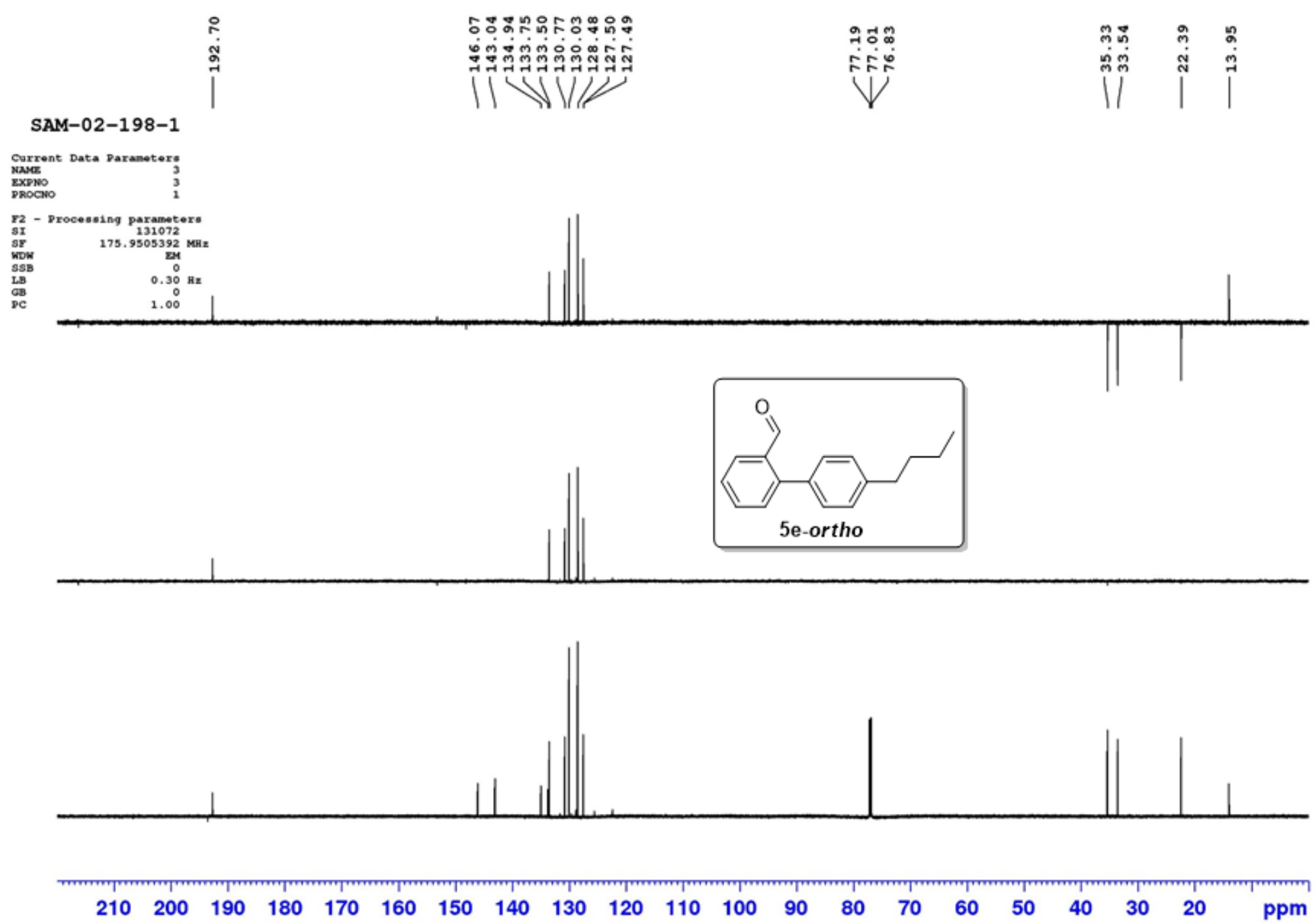
Solvent: $\mathrm{CDCl}_{3}$

SFO1: $700 \mathrm{MHz}$

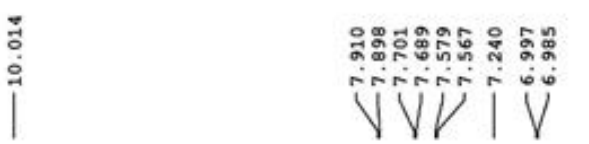

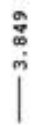

|

SAM-02-191-2

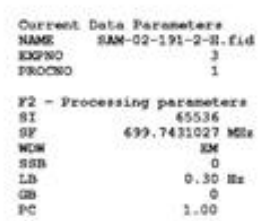
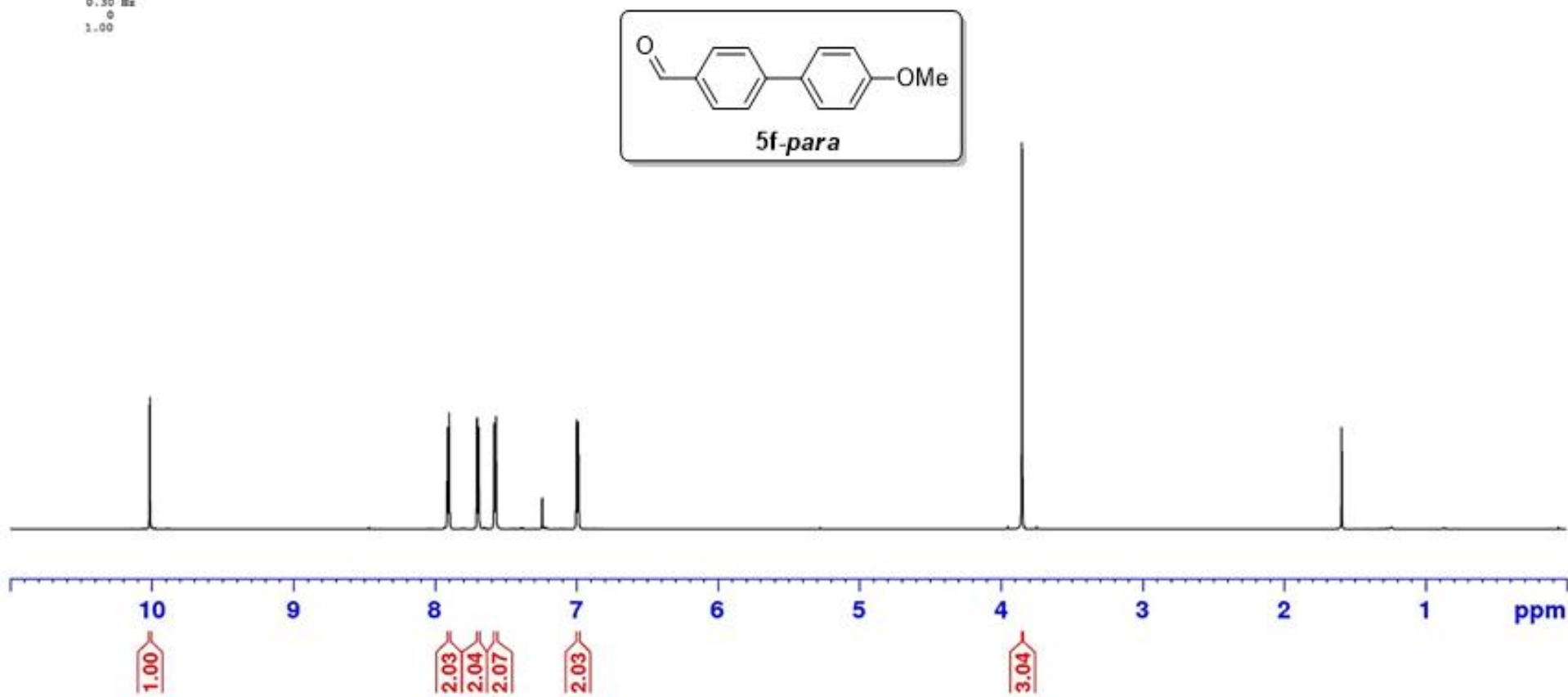
Solvent: $\mathrm{CDCl}_{3}$

SFO1: $175 \mathrm{MHz}$

|

\section{SAM-02-191-2}
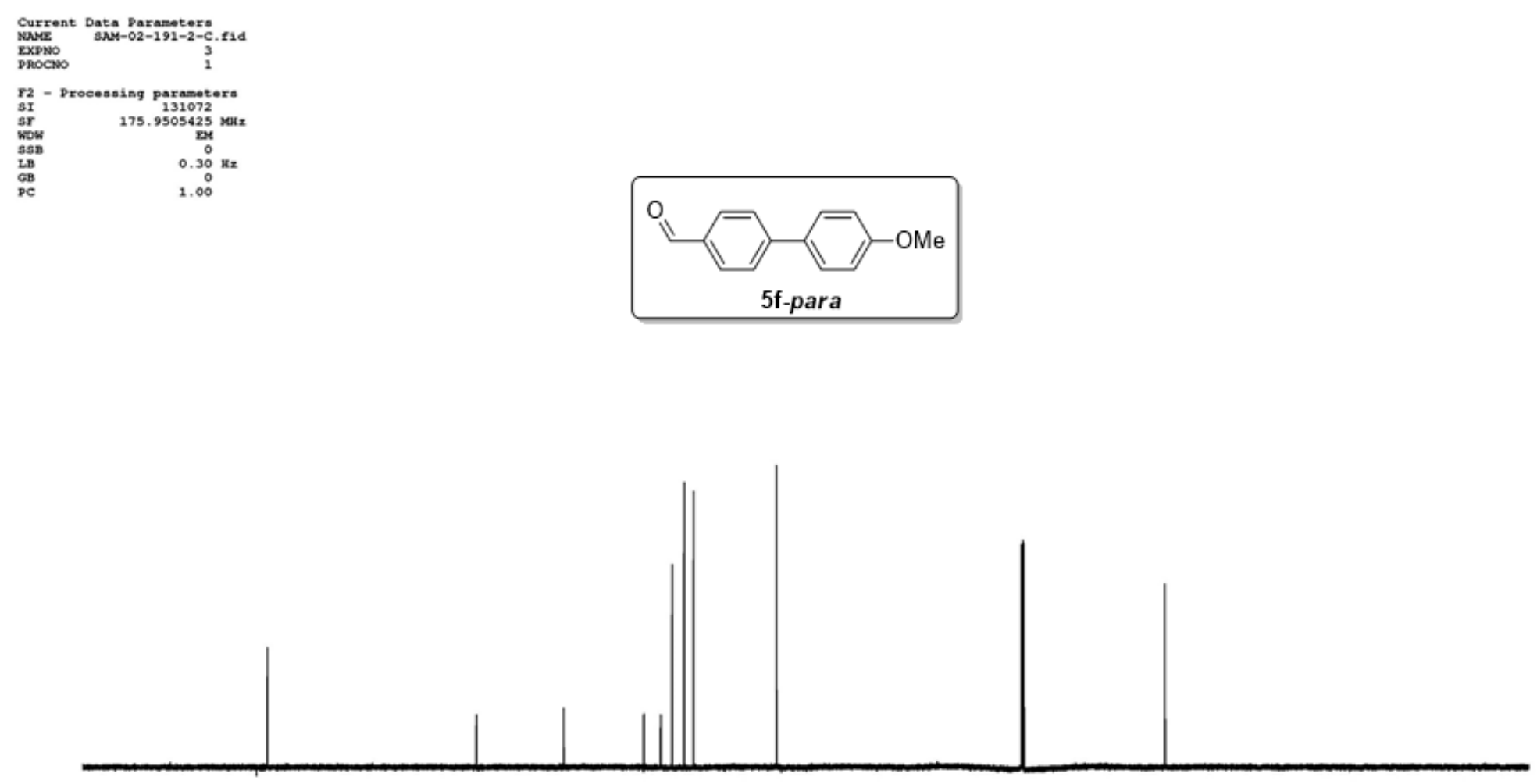

$\begin{array}{lllllllllllllllllllll}210 & 200 & 190 & 180 & 170 & 160 & 150 & 140 & 130 & 120 & 110 & 100 & 90 & 80 & 70 & 60 & 50 & 40 & 30 & 20 & \mathrm{ppm}\end{array}$ 
Solvent: $\mathrm{CDCl}_{3}$

SFO1: $700 \mathrm{MHz}$

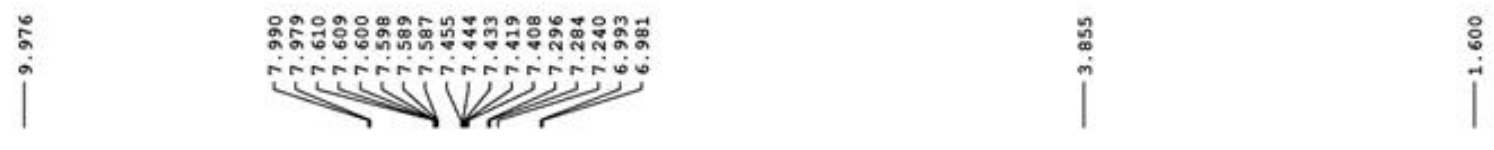

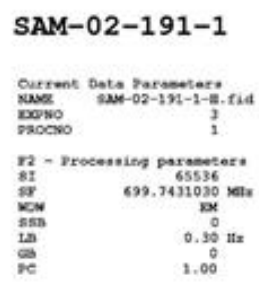

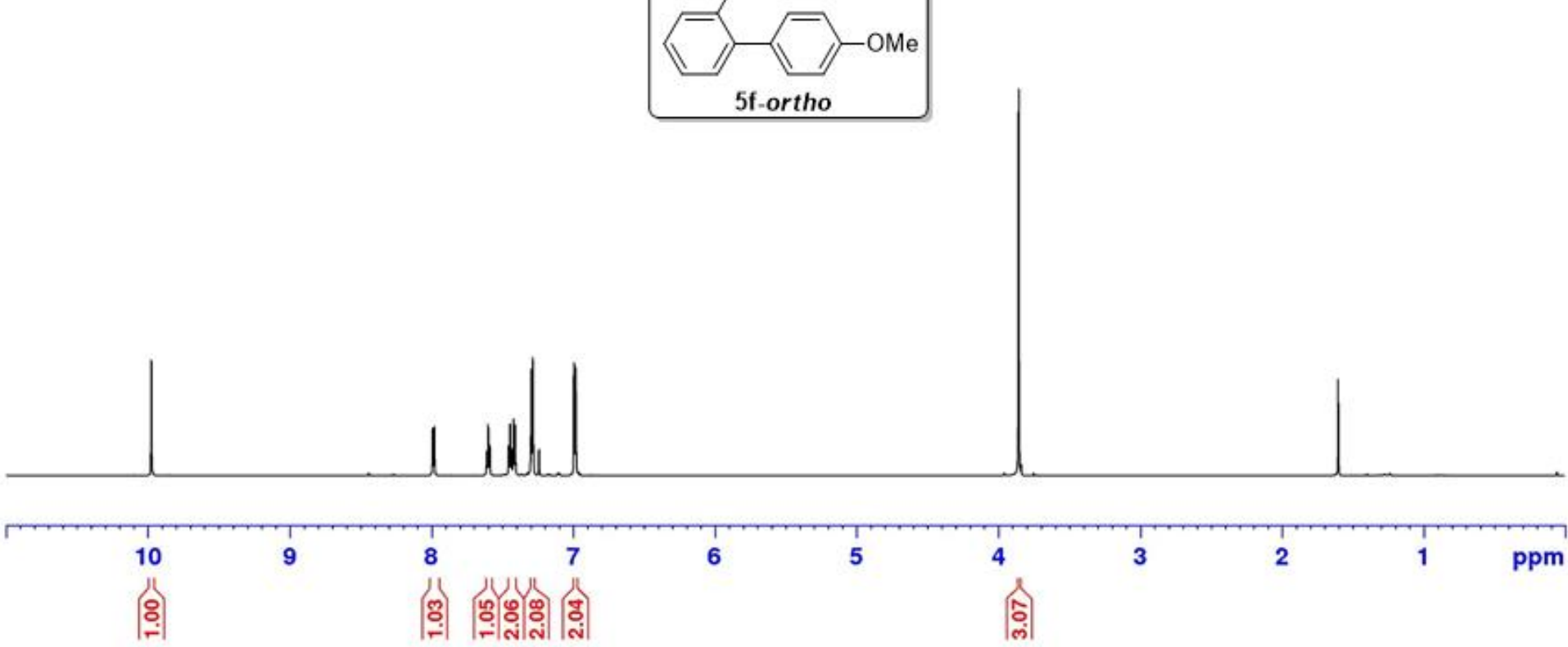


Solvent: $\mathrm{CDCl}_{3}$

SFO1: $175 \mathrm{MHz}$

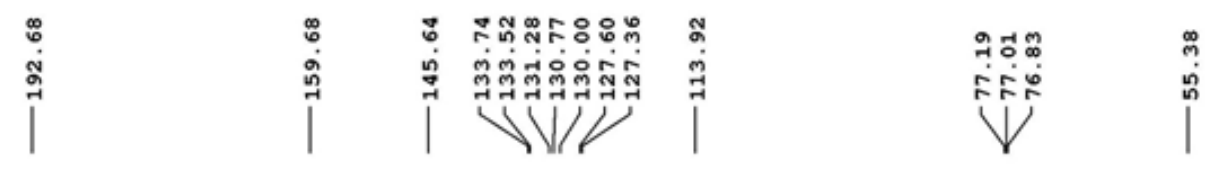

\section{SAM-02-191-1}
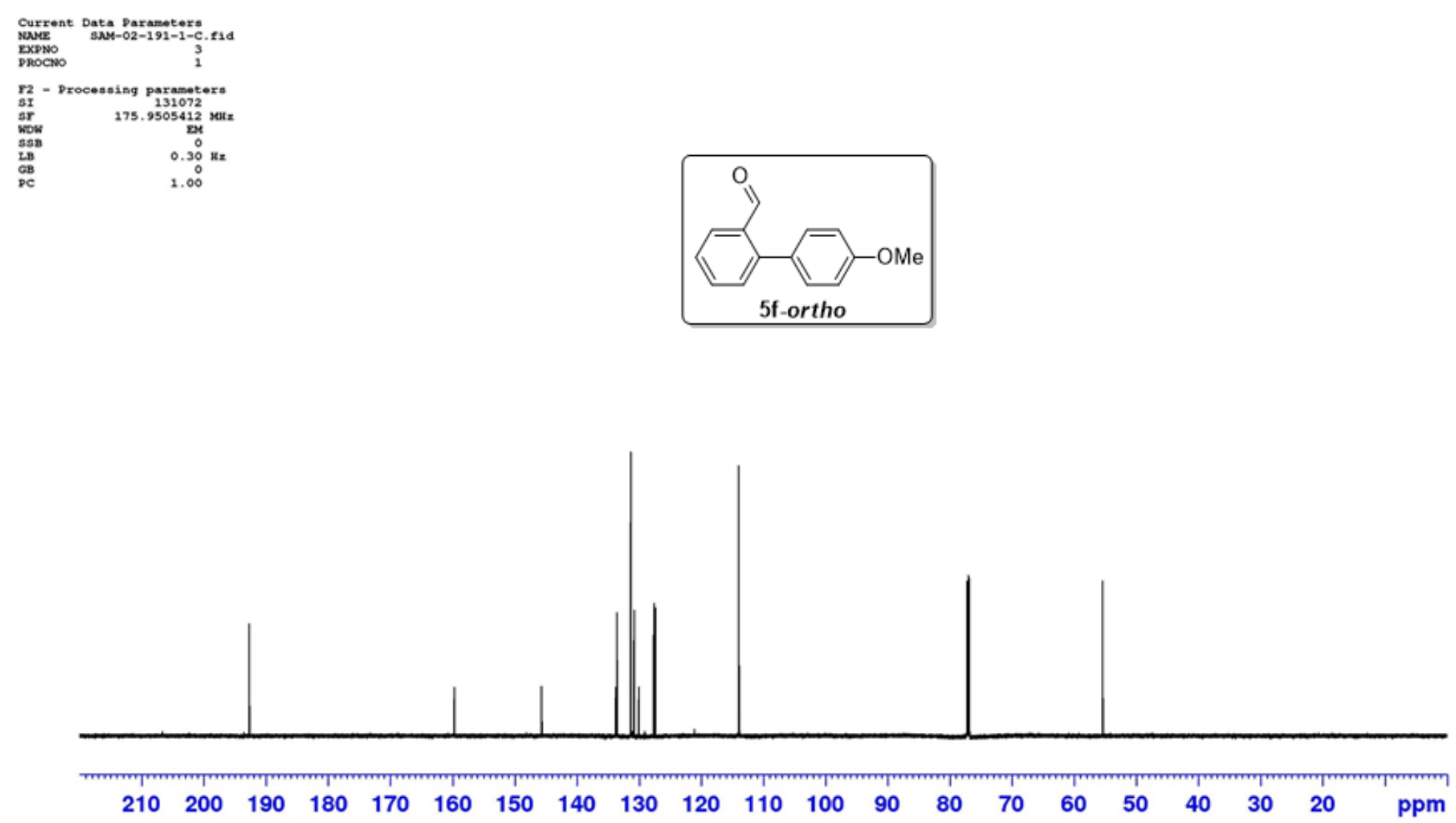
Solvent: $\mathrm{CDCl}_{3}$

SFO1: $700 \mathrm{MHz}$

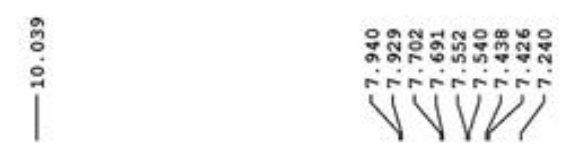

SAM-02-176-2

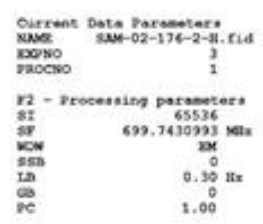
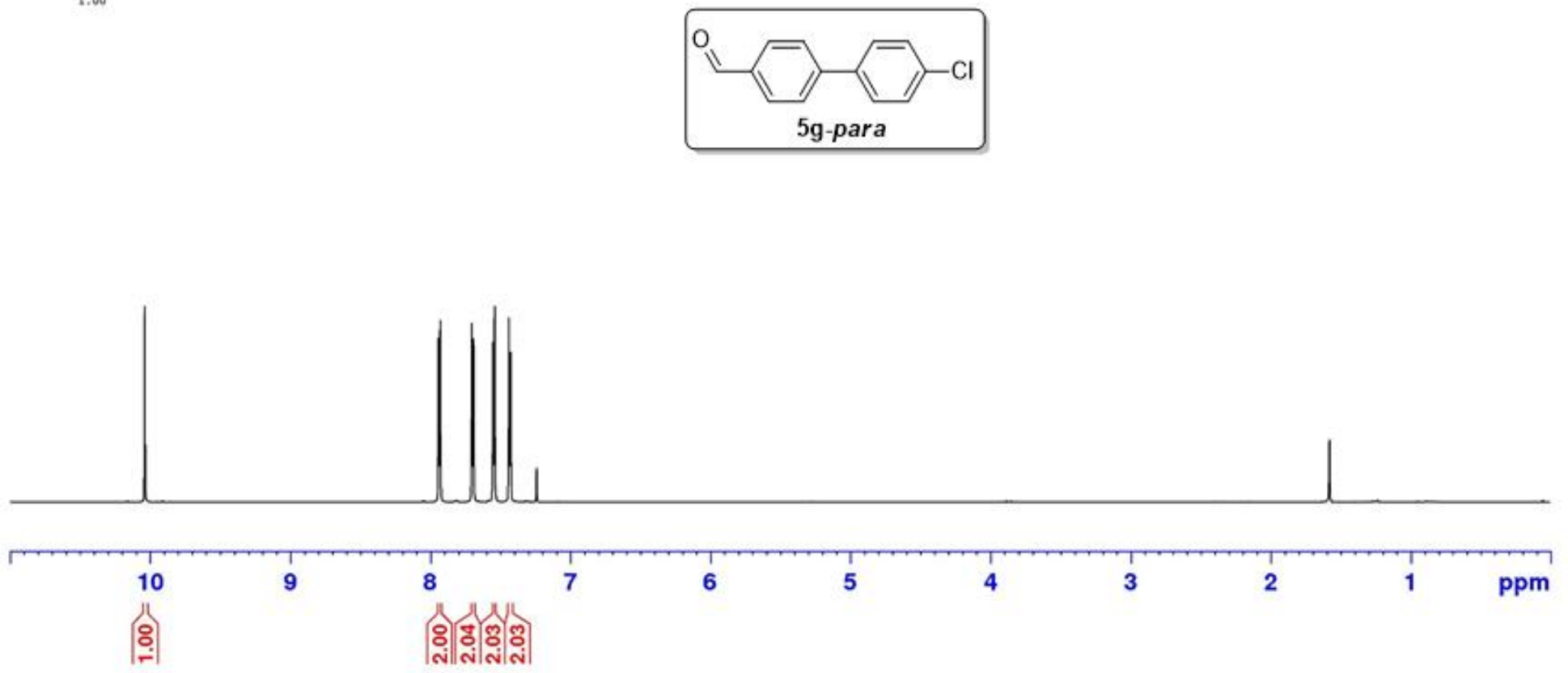
Solvent: $\mathrm{CDCl}_{3}$

SFO1: $175 \mathrm{MHz}$

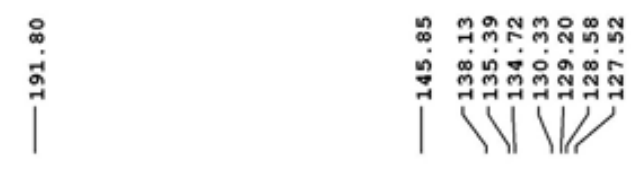

$\stackrel{-\infty}{\circ}$

Fह

SAM-02-176-2
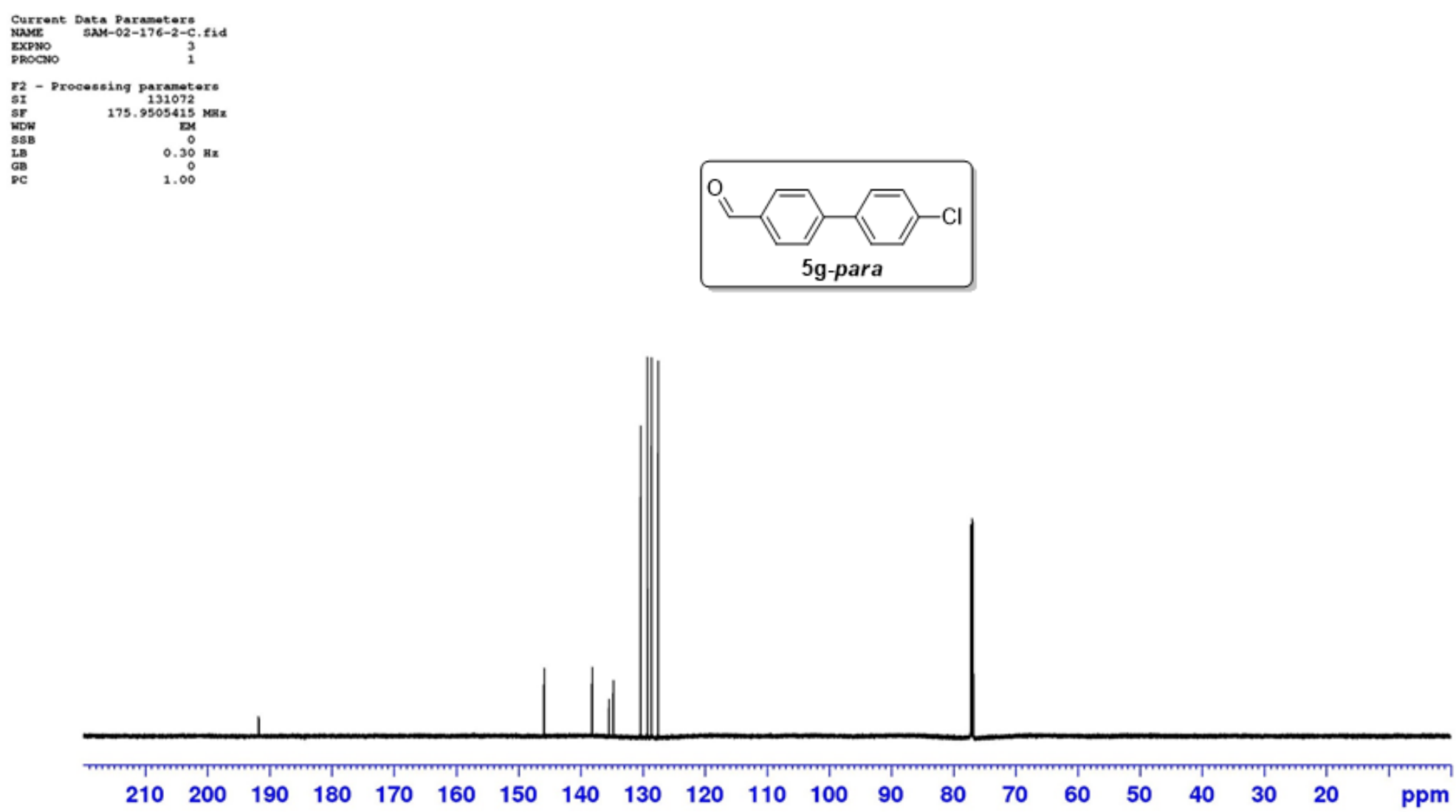
Solvent: $\mathrm{CDCl}_{3}$

SFO1: $700 \mathrm{MHz}$

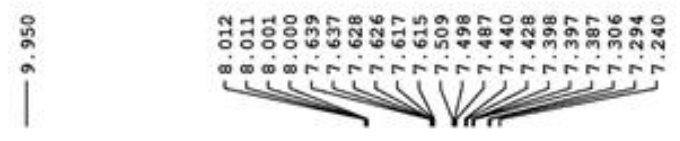

\section{SAM-02-176-1}

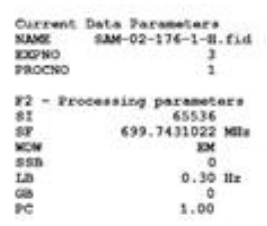
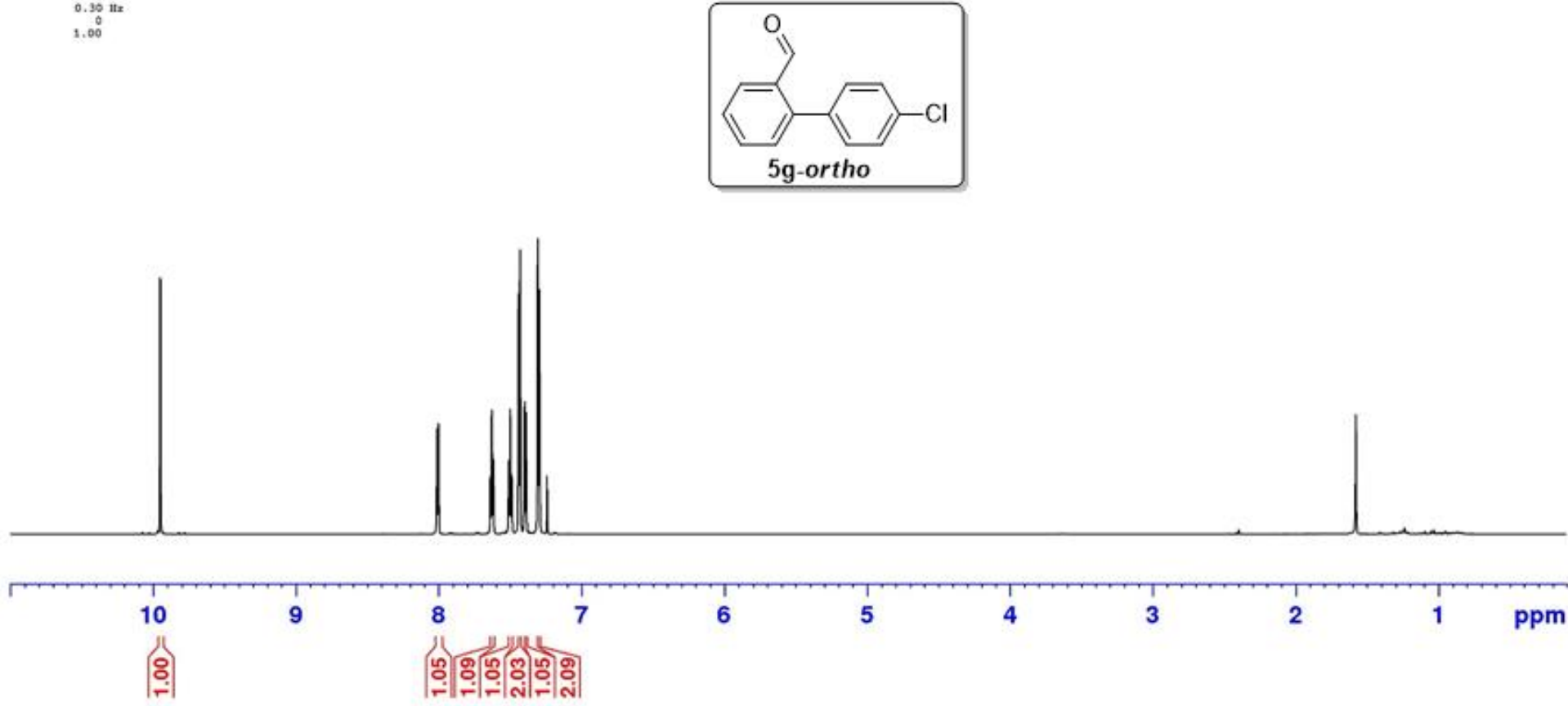
Solvent: $\mathrm{CDCl}_{3}$

SFO1: $175 \mathrm{MHz}$

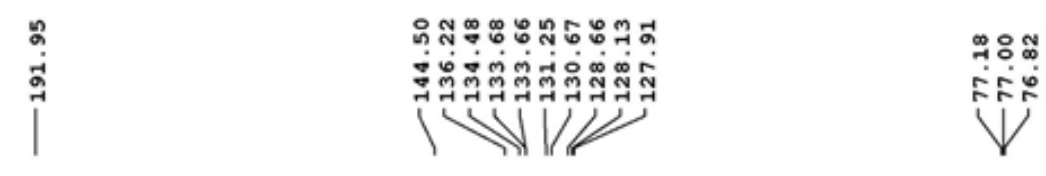

SAM-02-176-1
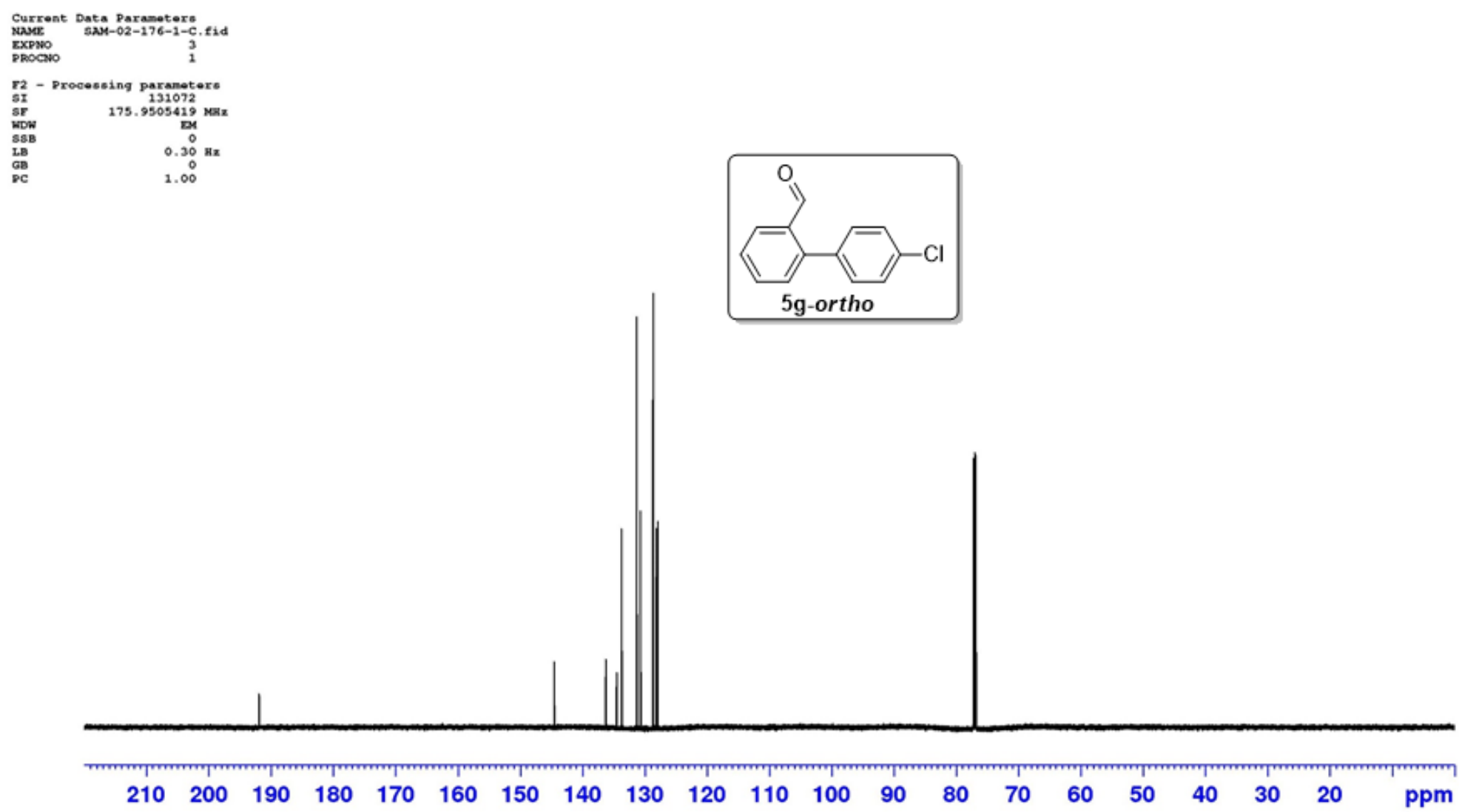
Solvent: $\mathrm{CDCl}_{3}$

SFO1: $700 \mathrm{MHz}$

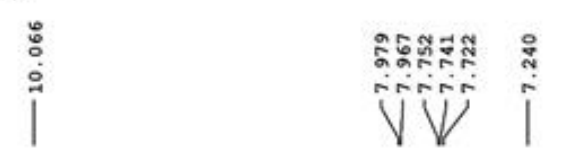

$\stackrel{\circ}{\stackrel{\leftrightarrow}{*}}$

SAM-02-196-2

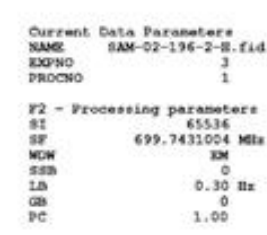
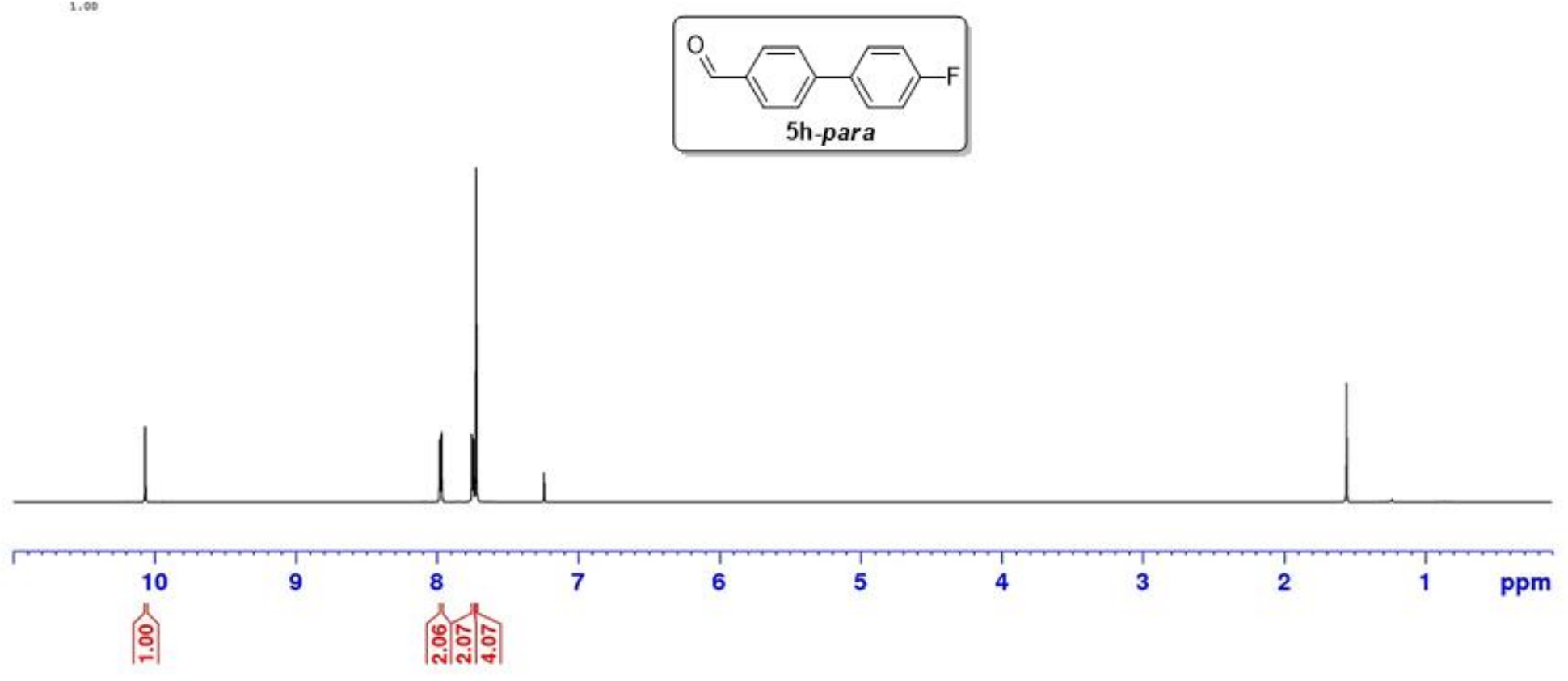
Solvent: $\mathrm{CDCl}_{3}$

SFO1: $175 \mathrm{MHz}$

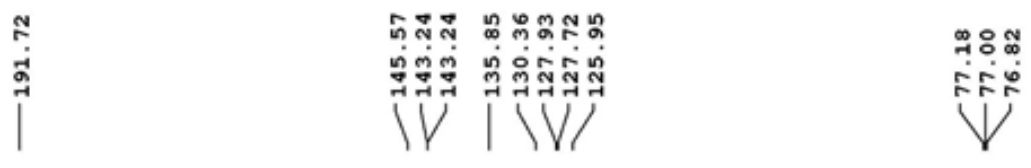

\section{SAM-02-196-2}

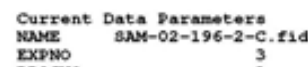

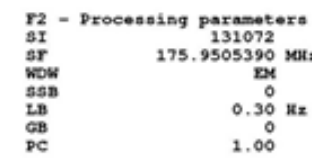
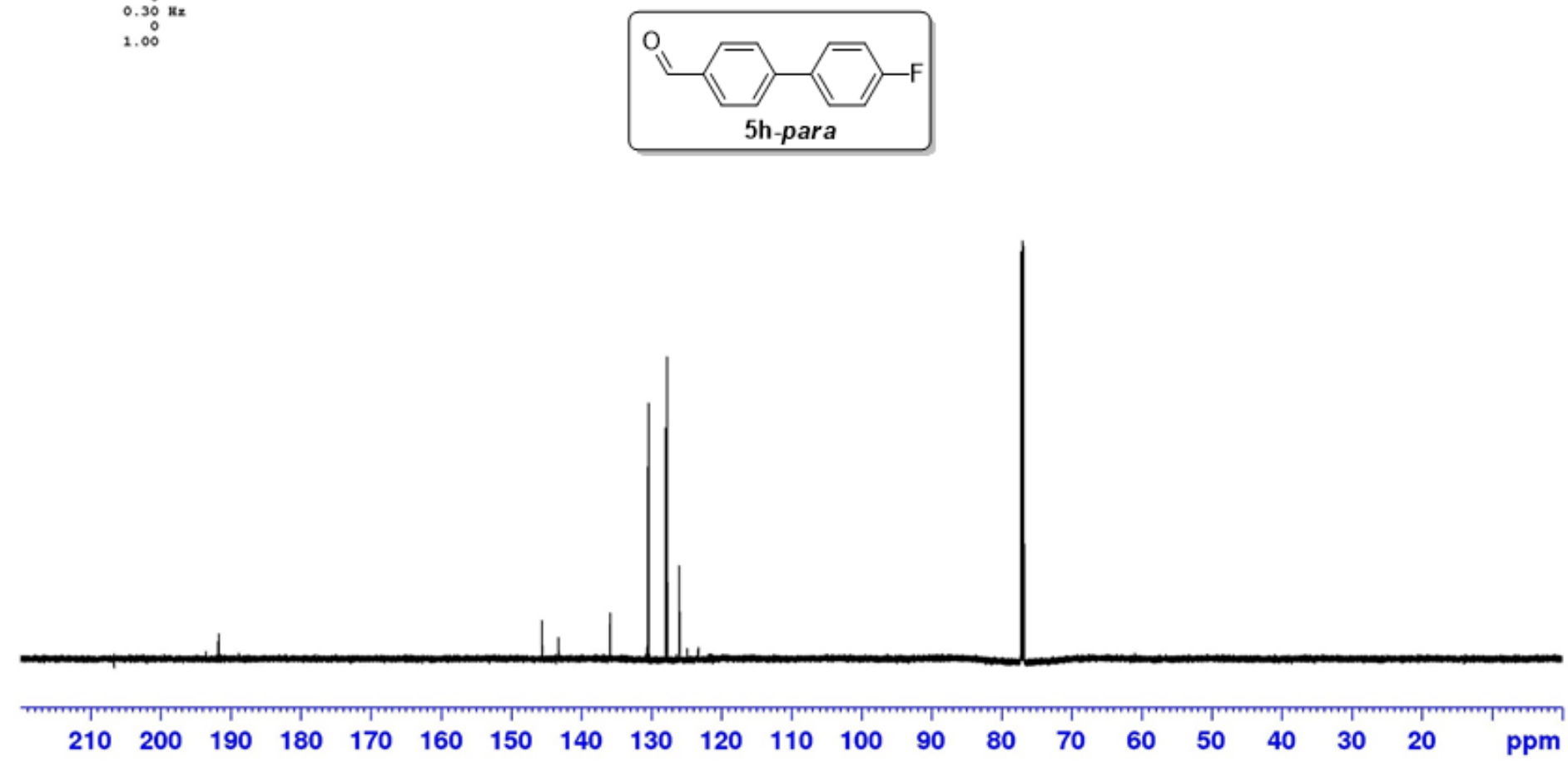
Solvent: $\mathrm{CDCl}_{3}$

SFO1: $700 \mathrm{MHz}$

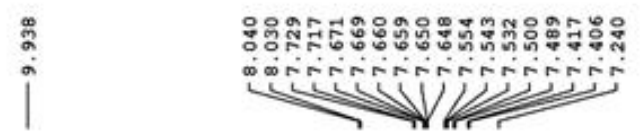

\section{SAM-02-196-1}
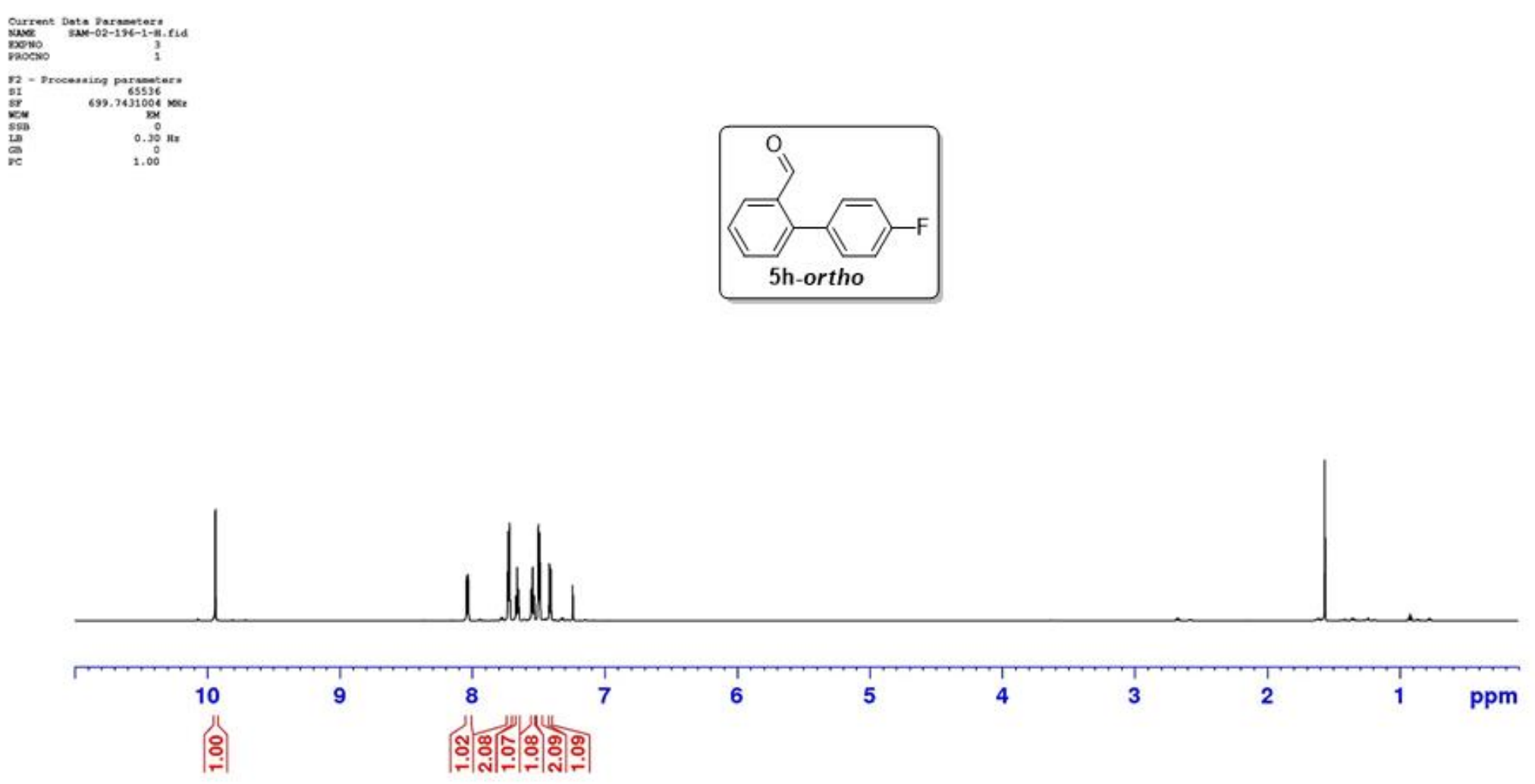
Solvent: $\mathrm{CDCl}_{3}$

SFO1: $175 \mathrm{MHz}$

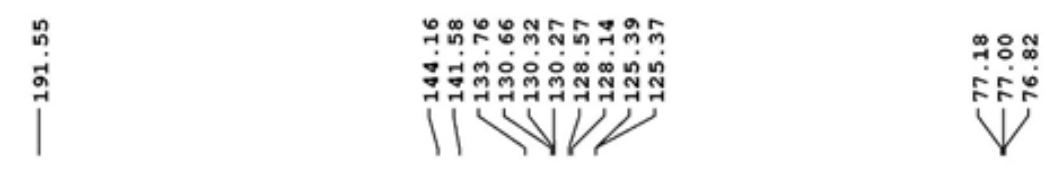

\section{SAM-02-196-1}
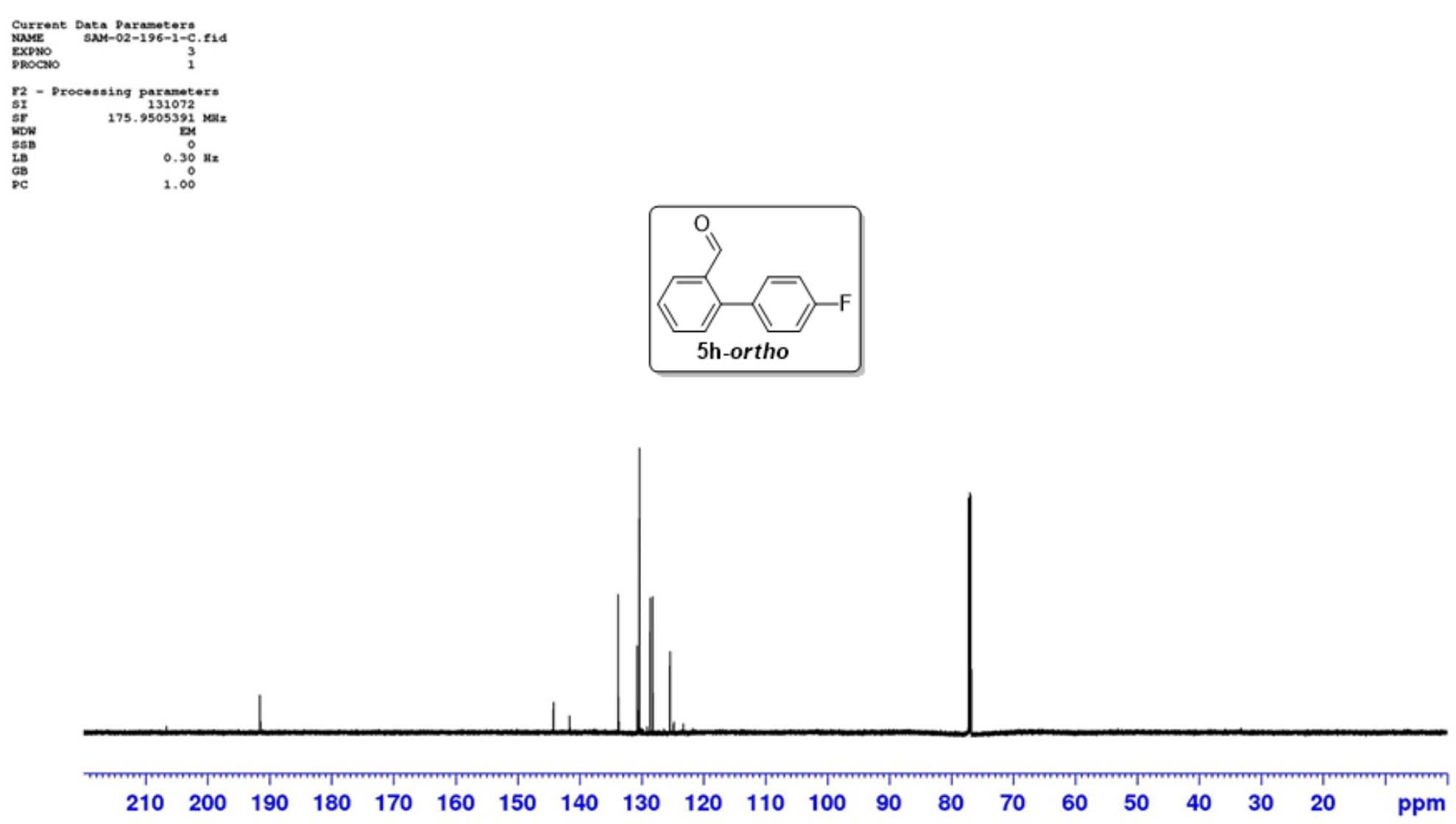
Solvent: $\mathrm{CDCl}_{3}$

SFO1: $700 \mathrm{MHz}$

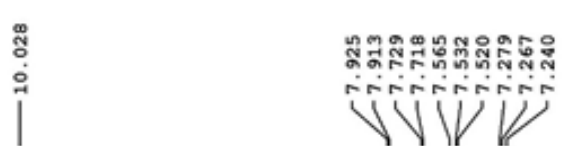

|

SAM-02-173-2

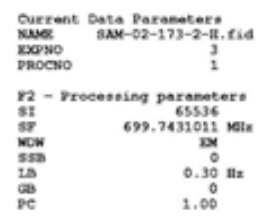
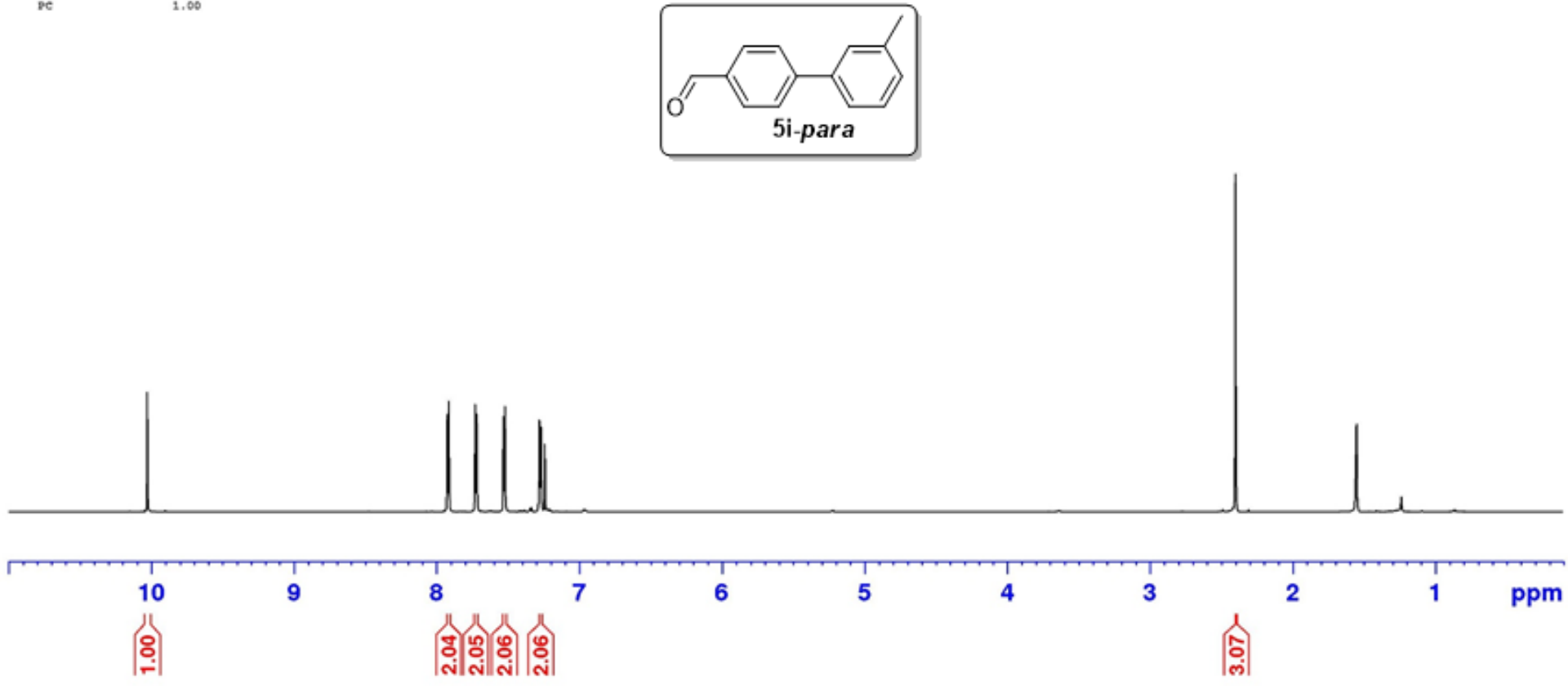
Solvent: $\mathrm{CDCl}_{3}$

SFO1: $175 \mathrm{MHz}$

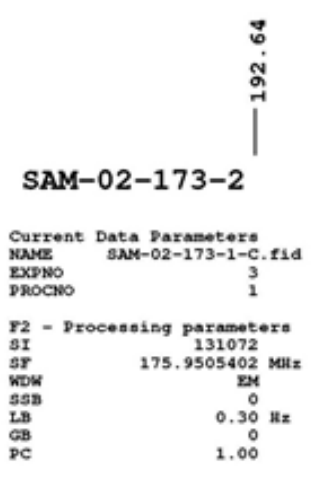

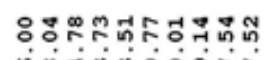

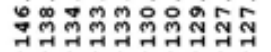

WVIV

$\underset{\sim}{\infty}:$

FF

$\stackrel{\infty}{7}$

V

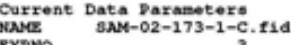

PXOCONO
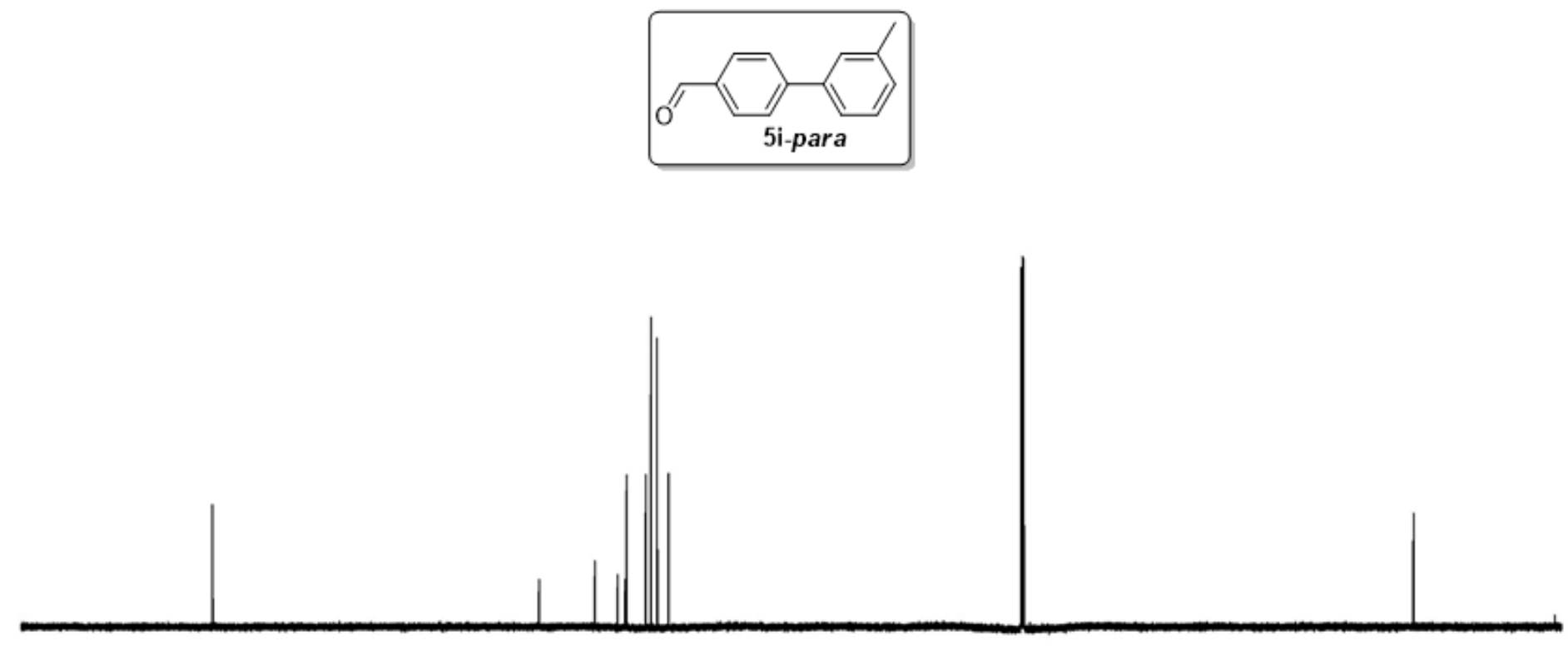

$\begin{array}{lllllllllllllllllllll}210 & 200 & 190 & 180 & 170 & 160 & 150 & 140 & 130 & 120 & 110 & 100 & 90 & 80 & 70 & 60 & 50 & 40 & 30 & 20 & \mathrm{ppm}\end{array}$ 
Solvent: $\mathrm{CDCl}_{3}$

SFO1: $700 \mathrm{MHz}$

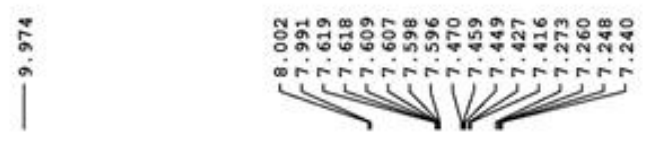

|

SAM-02-173-1

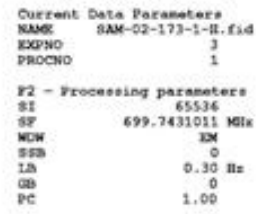

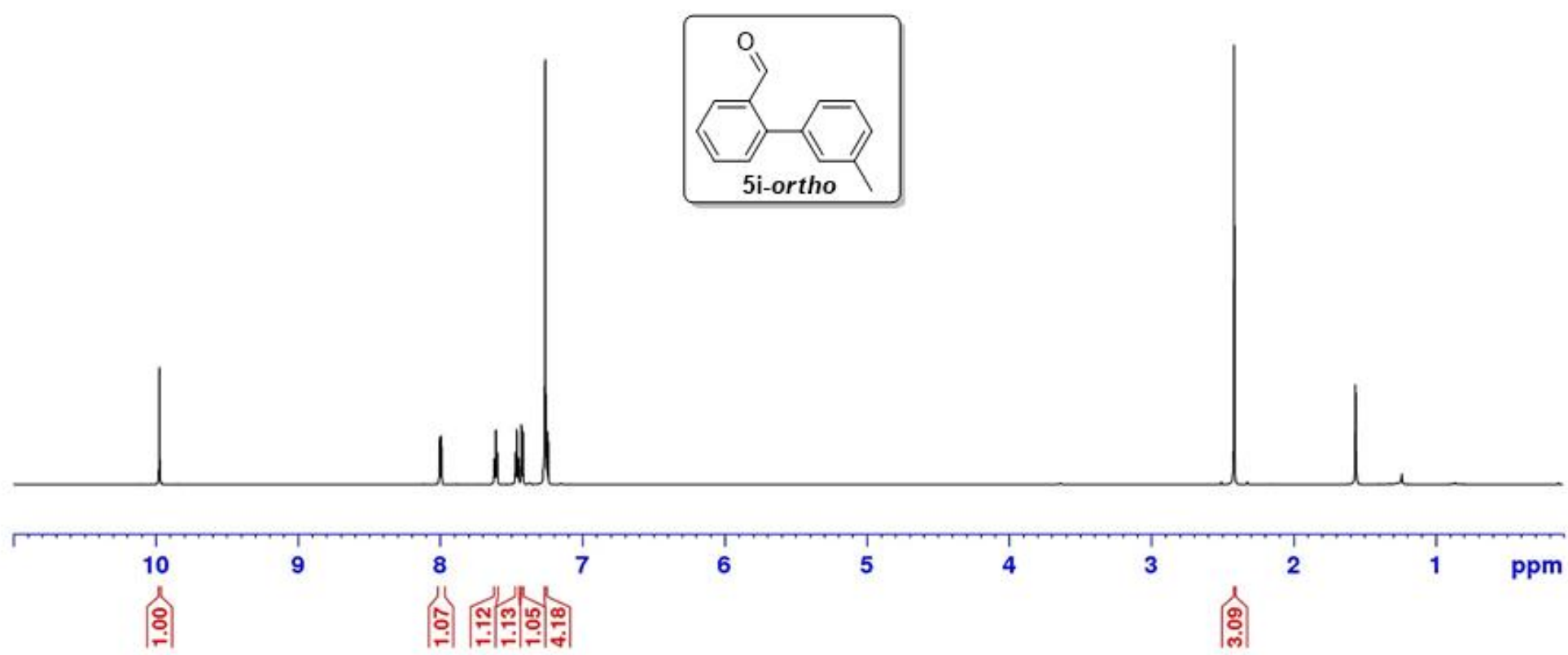


Solvent: $\mathrm{CDCl}_{3}$

SFO1: $175 \mathrm{MHz}$

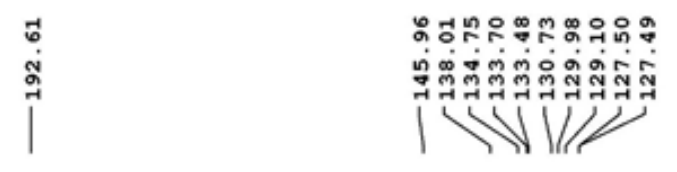

SAM-02-173-1
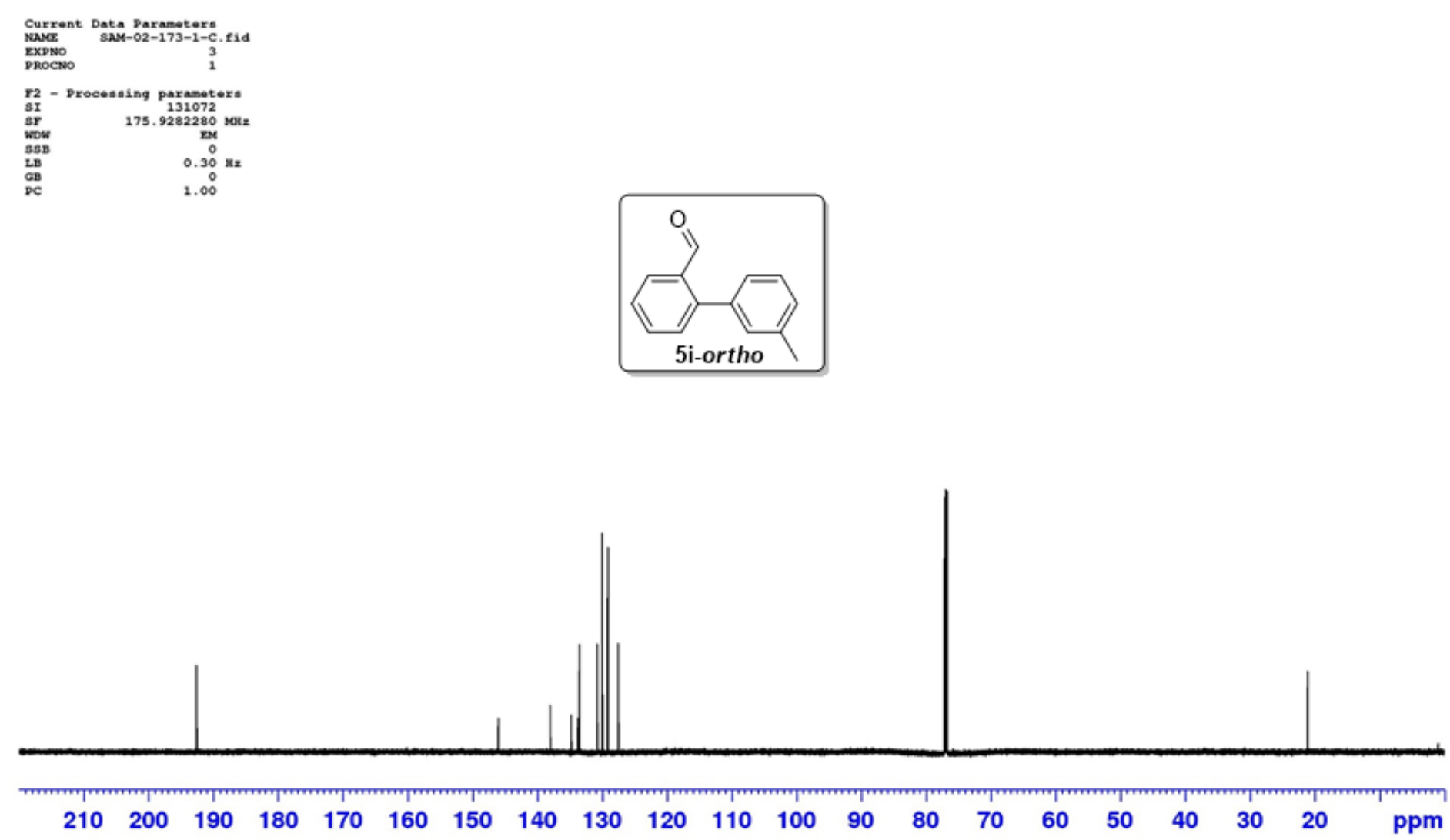
Solvent: $\mathrm{CDCl}_{3}$

SFO1: $700 \mathrm{MHz}$
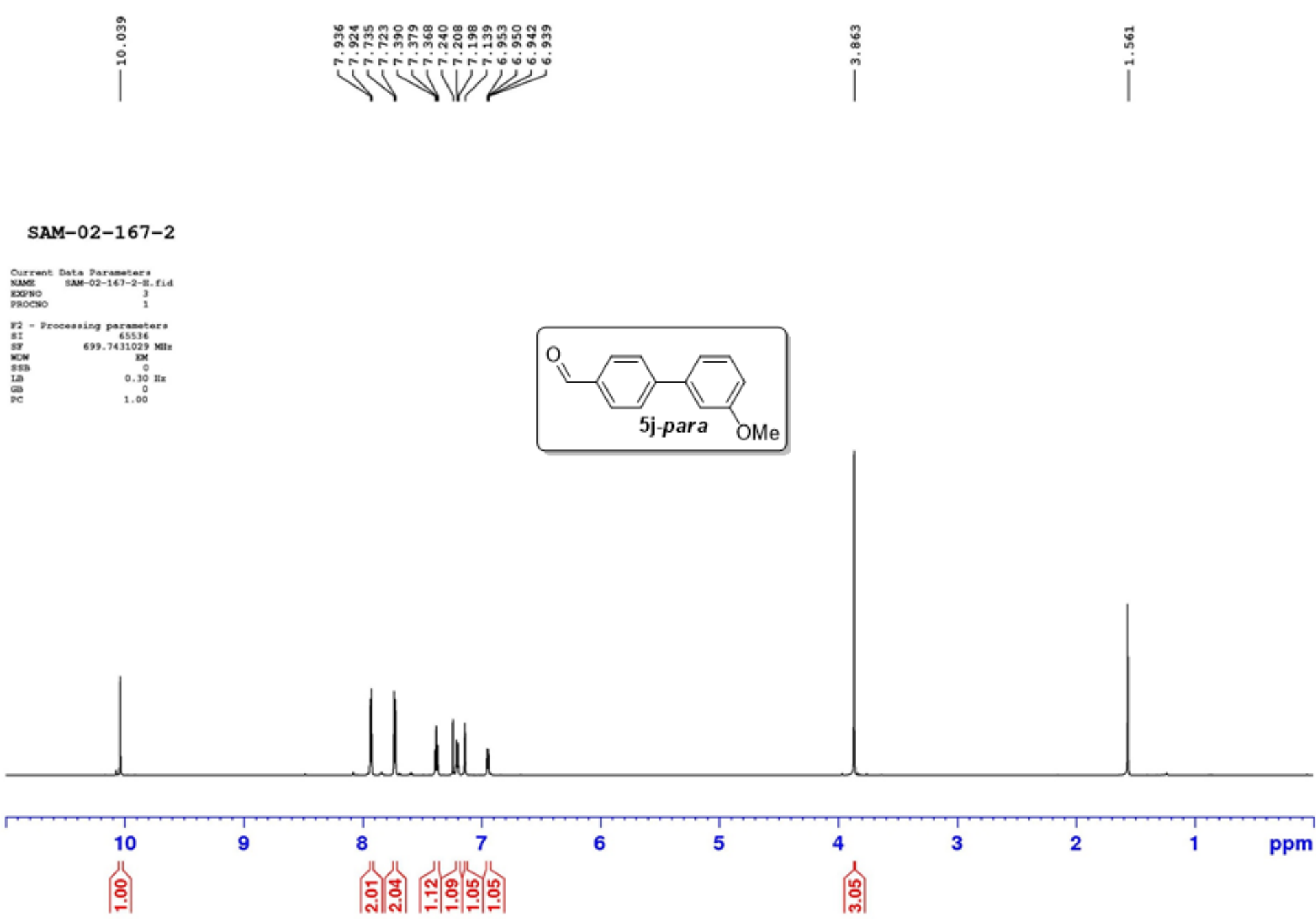
Solvent: $\mathrm{CDCl}_{3}$

SFO1: $175 \mathrm{MHz}$

|

\section{SAM-02-167-2}

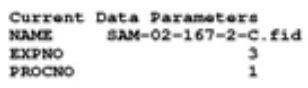

res - Procossing parasooters

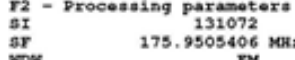

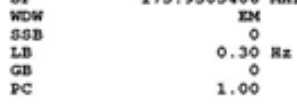

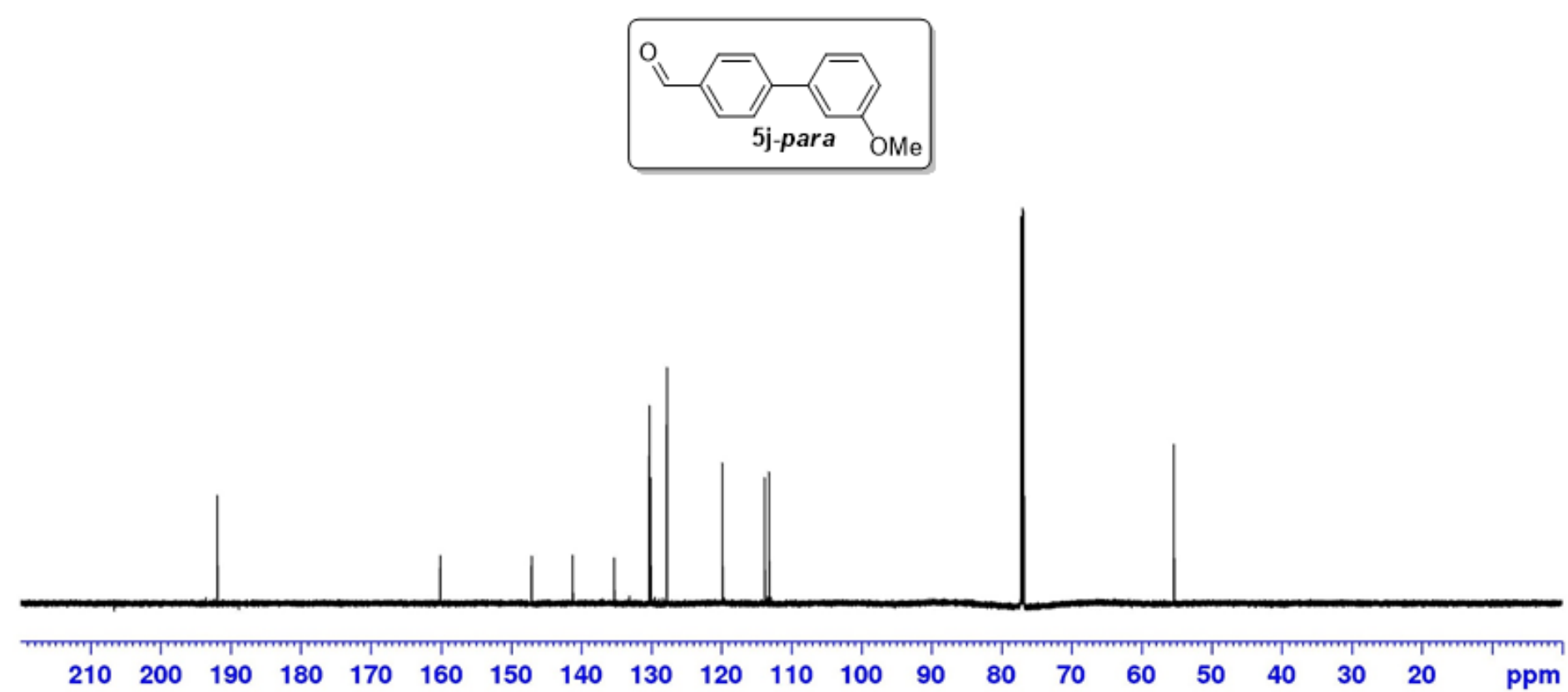


Solvent: $\mathrm{CDCl}_{3}$

SFO1: $700 \mathrm{MHz}$
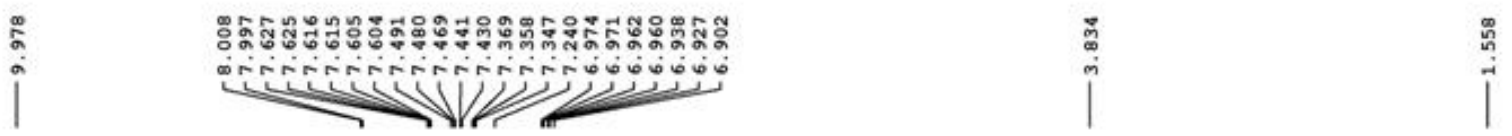

SAM-02-167-1

Purtant Bara Parasotor:

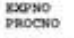

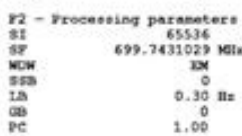

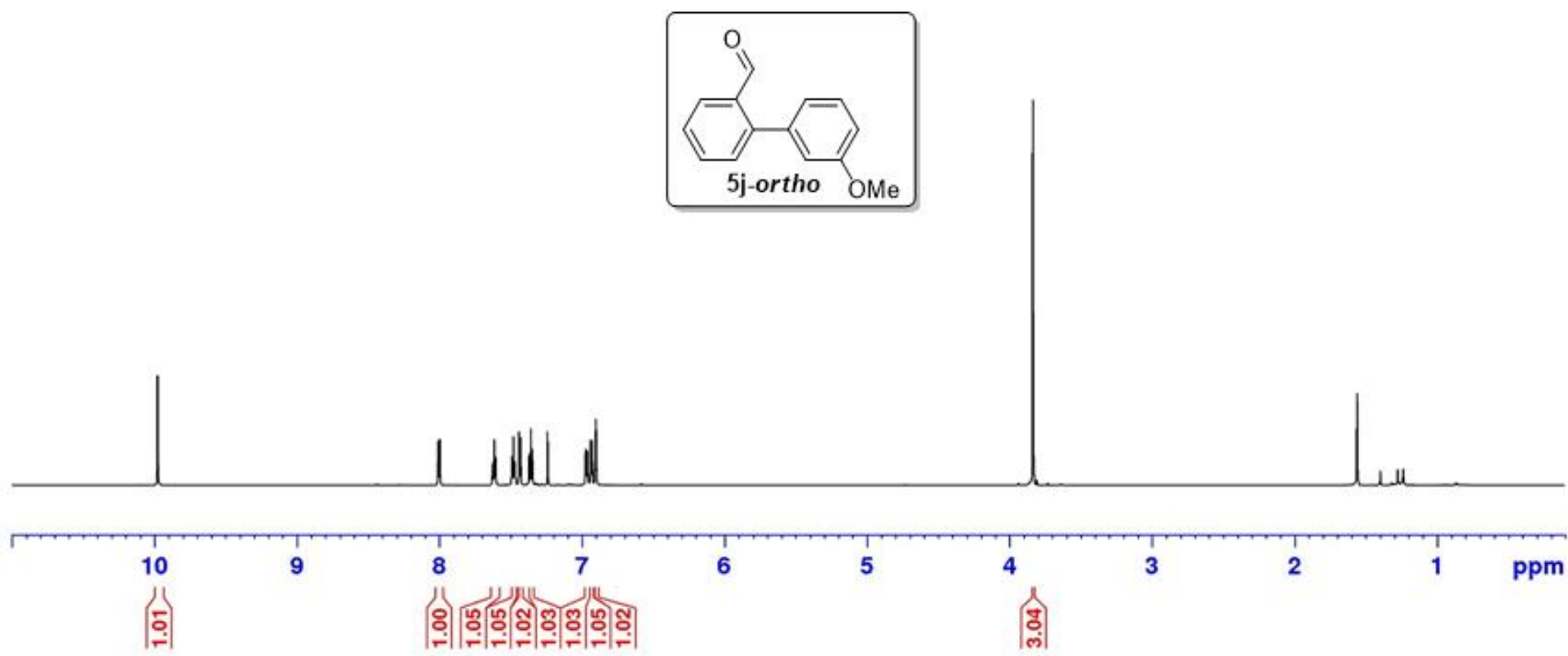


Solvent: $\mathrm{CDCl}_{3}$

SFO1: $175 \mathrm{MHz}$

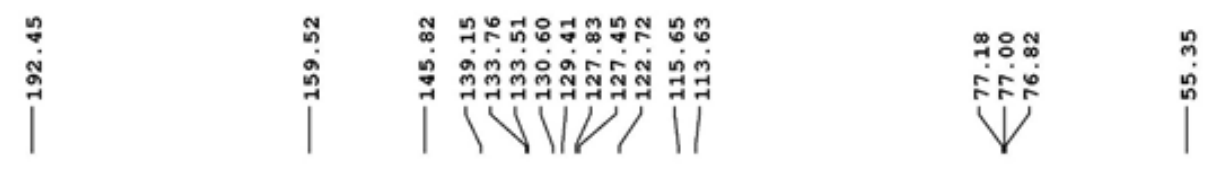

SAM-02-167-1

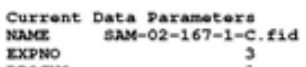

PRPOCNO
PRONO

$r_{3}^{2}$ - processing parasoters

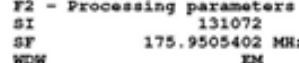

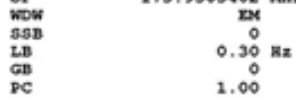
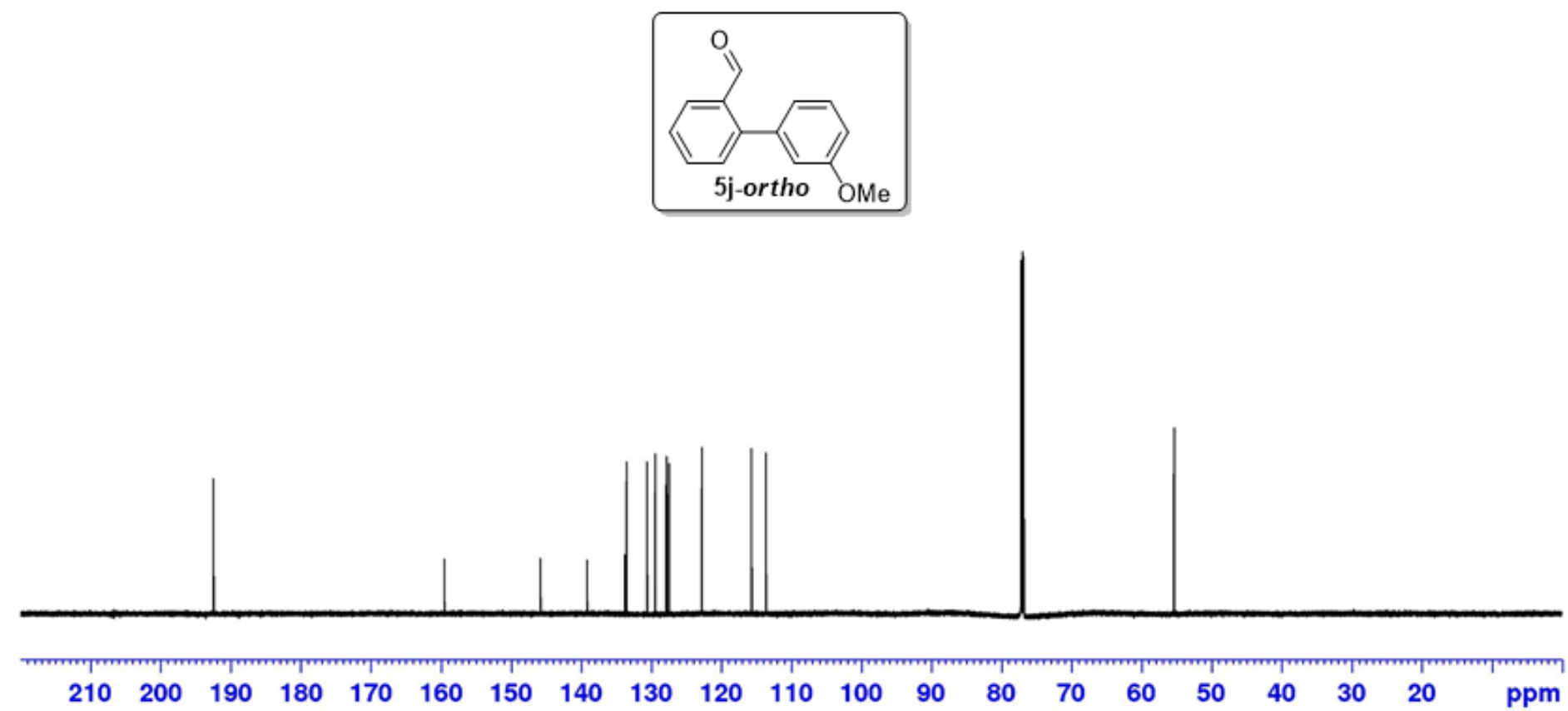
Solvent: $\mathrm{CDCl}_{3}$

SFO1: $700 \mathrm{MHz}$

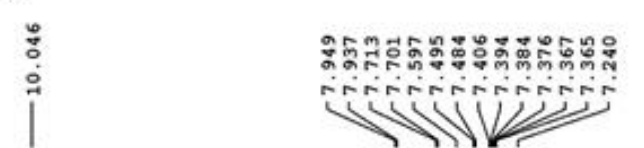

\section{SAM-02-172-2}

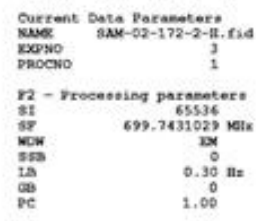
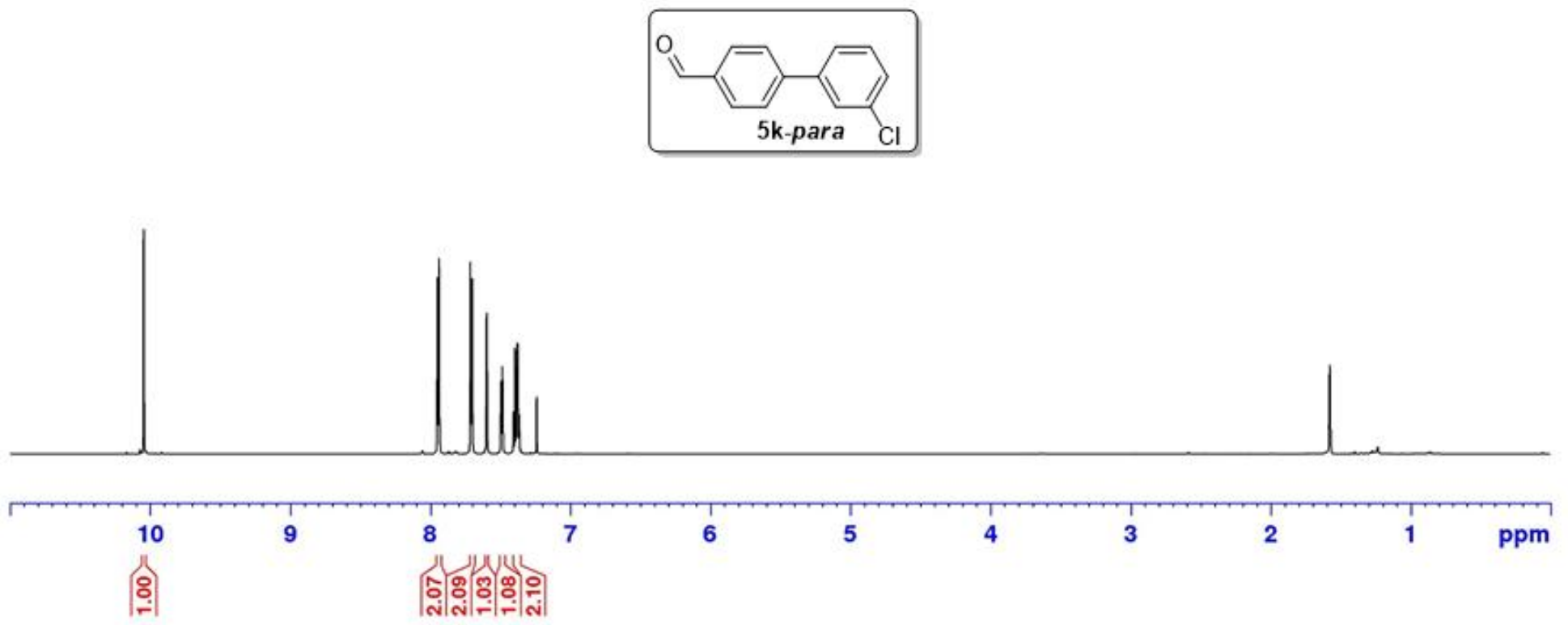
Solvent: $\mathrm{CDCl}_{3}$

SFO1: $175 \mathrm{MHz}$

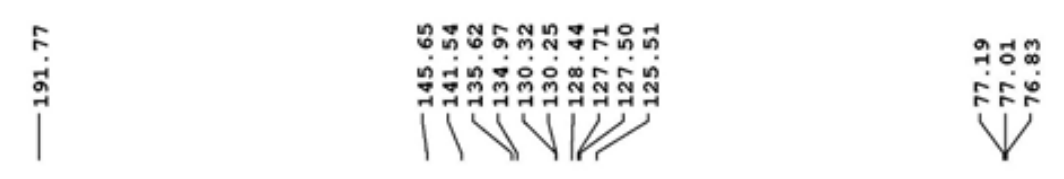

\section{SAM-02-172-2}
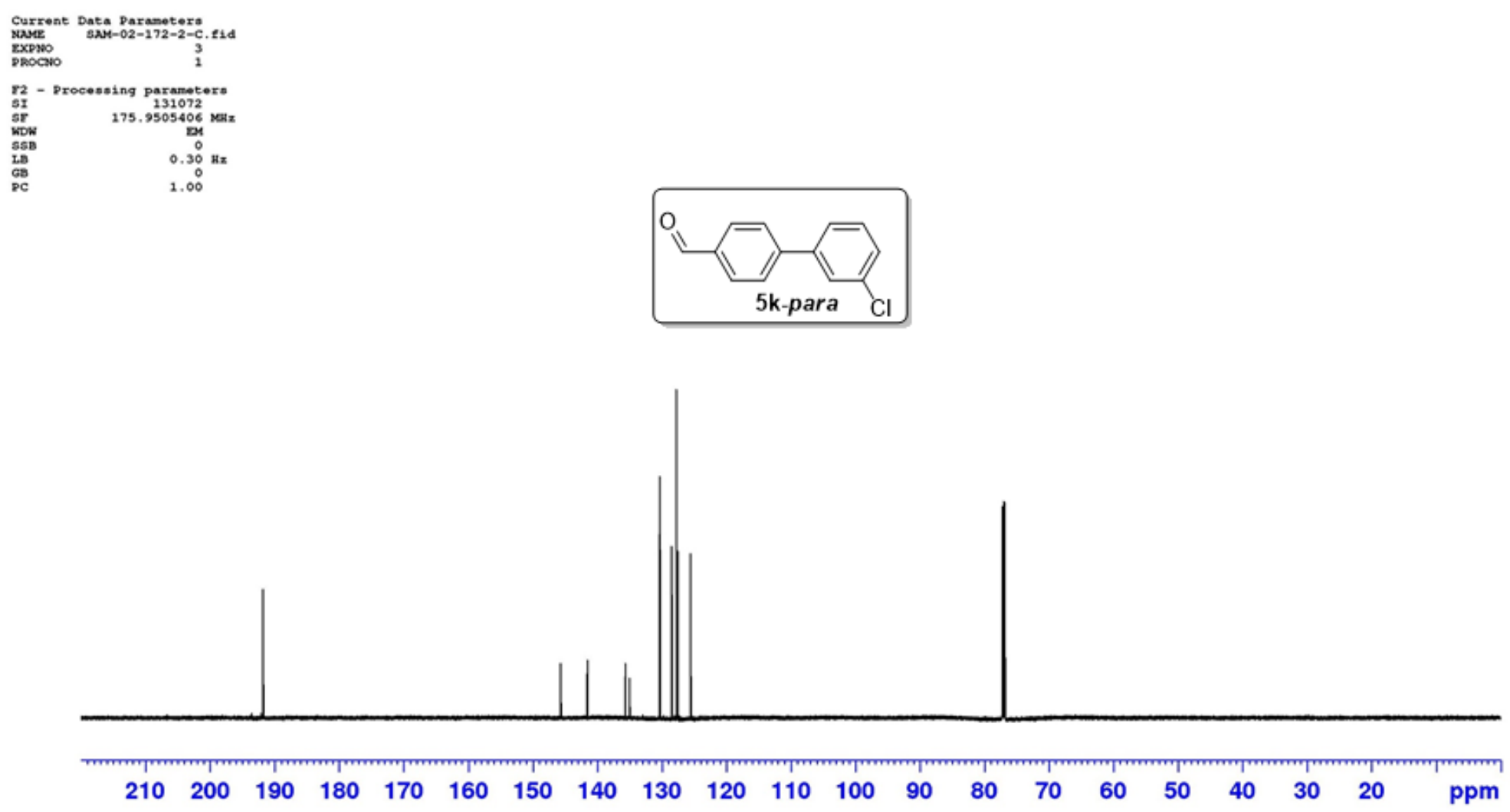
Solvent: $\mathrm{CDCl}_{3}$

SFO1: $700 \mathrm{MHz}$

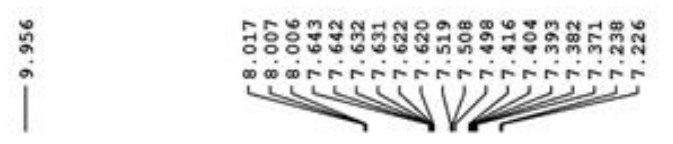

SAM-02-172-1

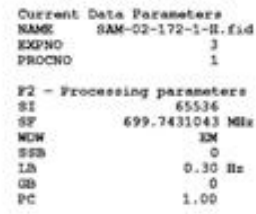
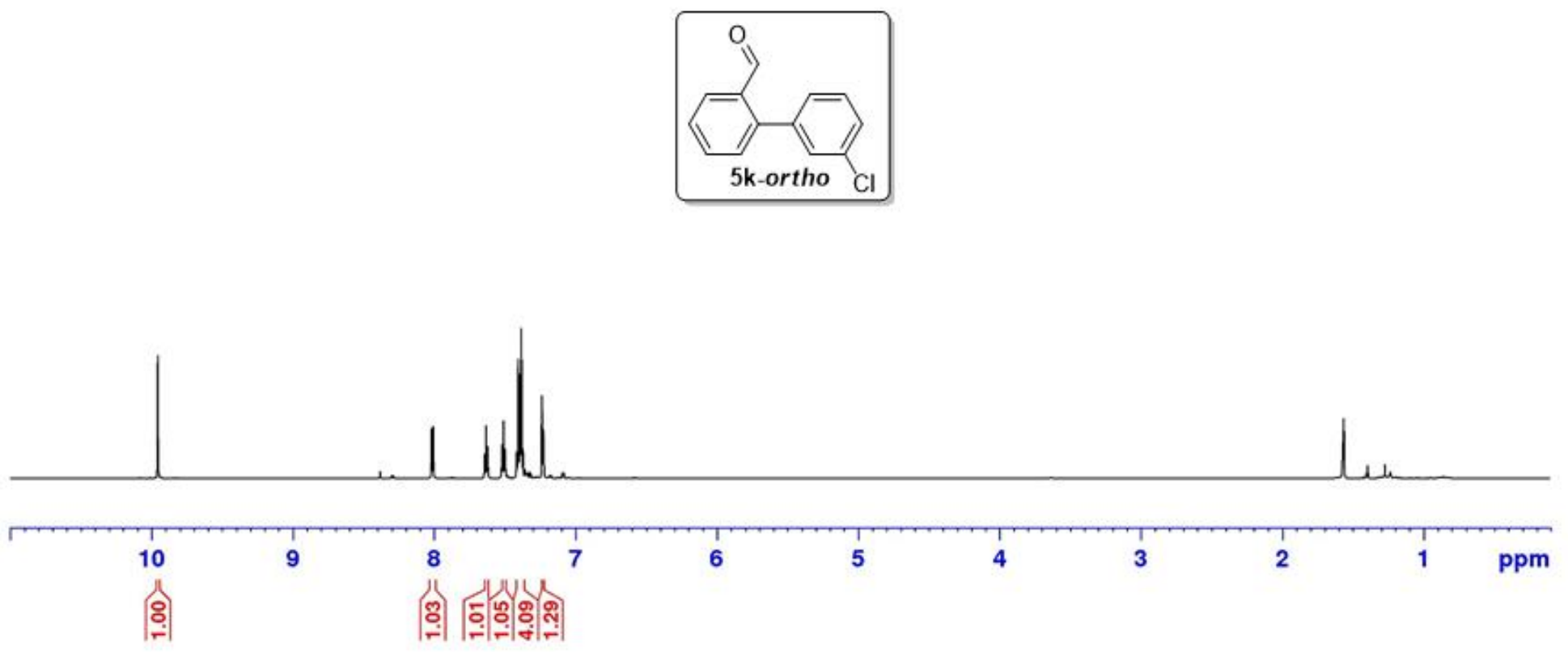
Solvent: $\mathrm{CDCl}_{3}$

SFO1: $175 \mathrm{MHz}$

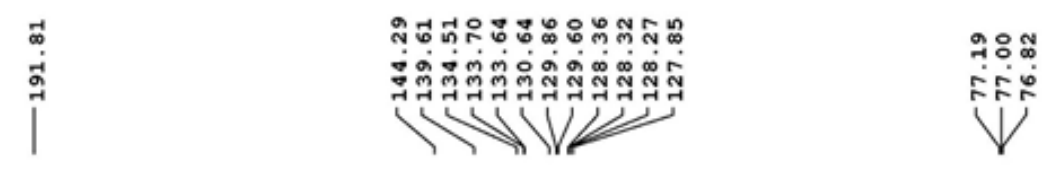

SAM-02-172-1
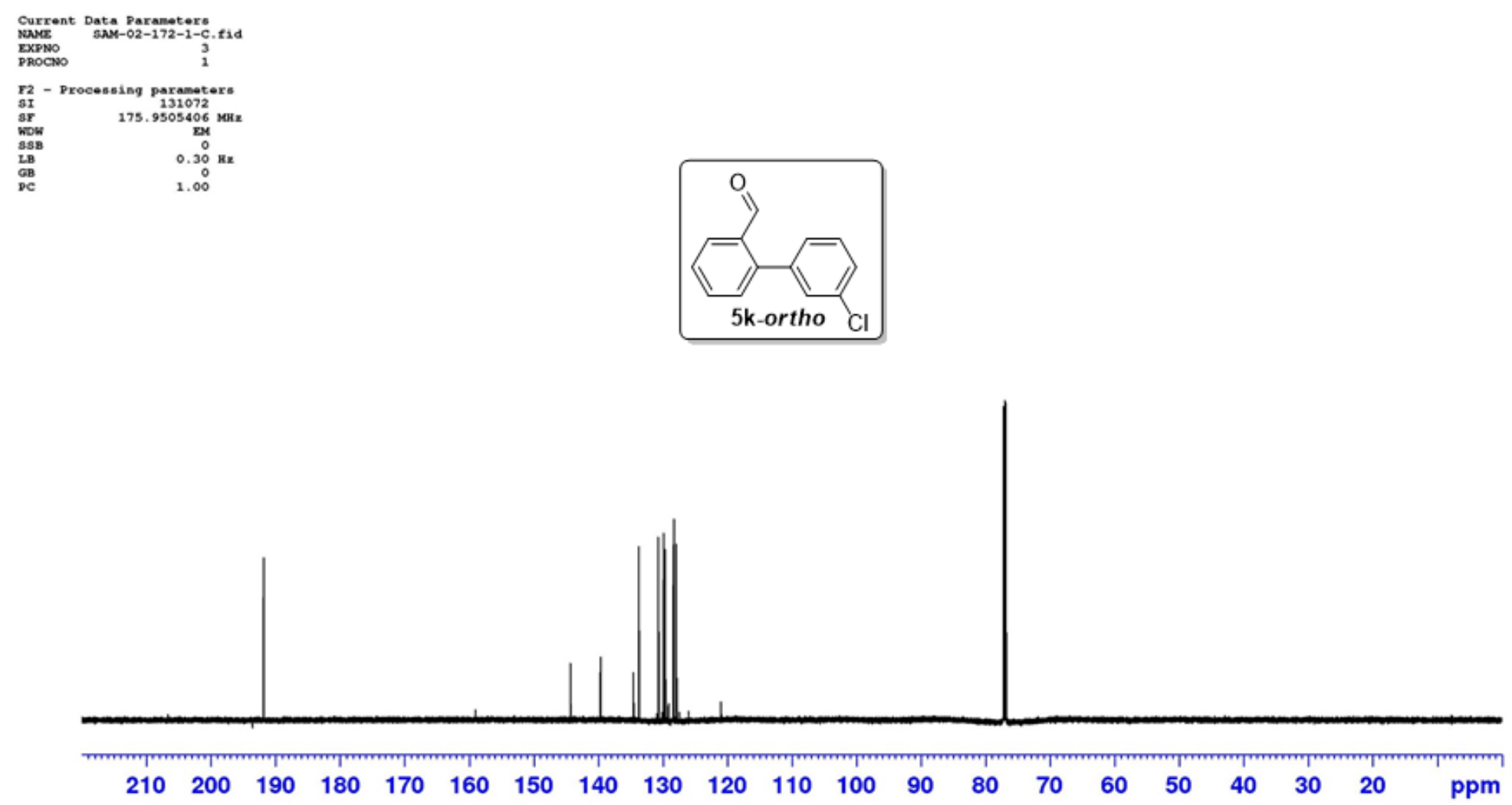
Solvent: $\mathrm{CDCl}_{3}$

SFO1: $700 \mathrm{MHz}$

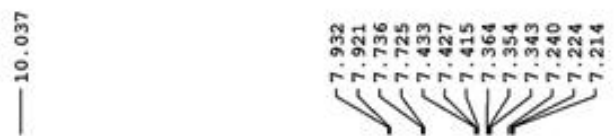

i

SAM-02-174-2

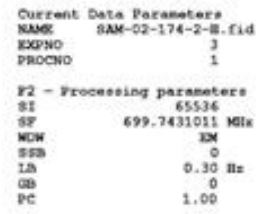
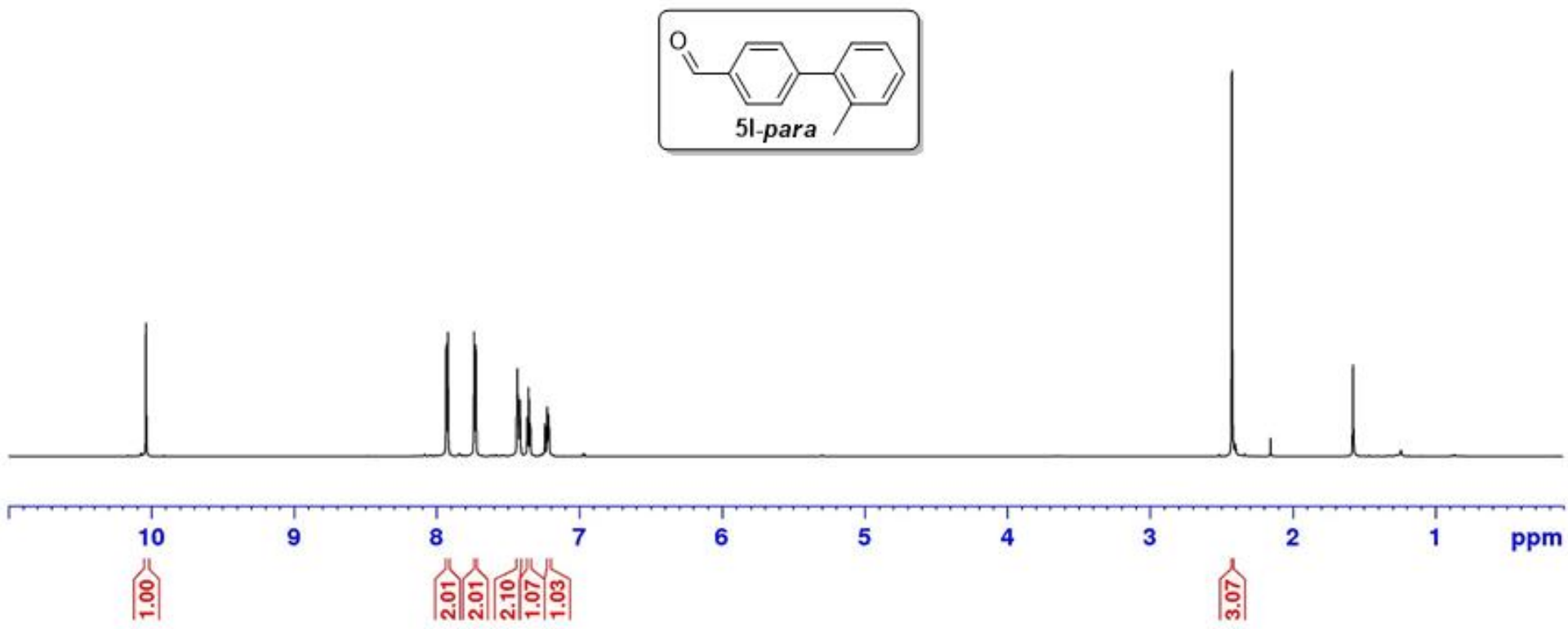
Solvent: $\mathrm{CDCl}_{3}$

SFO1: $175 \mathrm{MHz}$

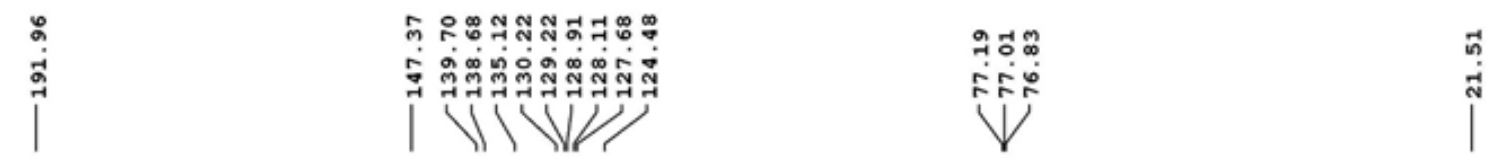

SAM-02-174-2
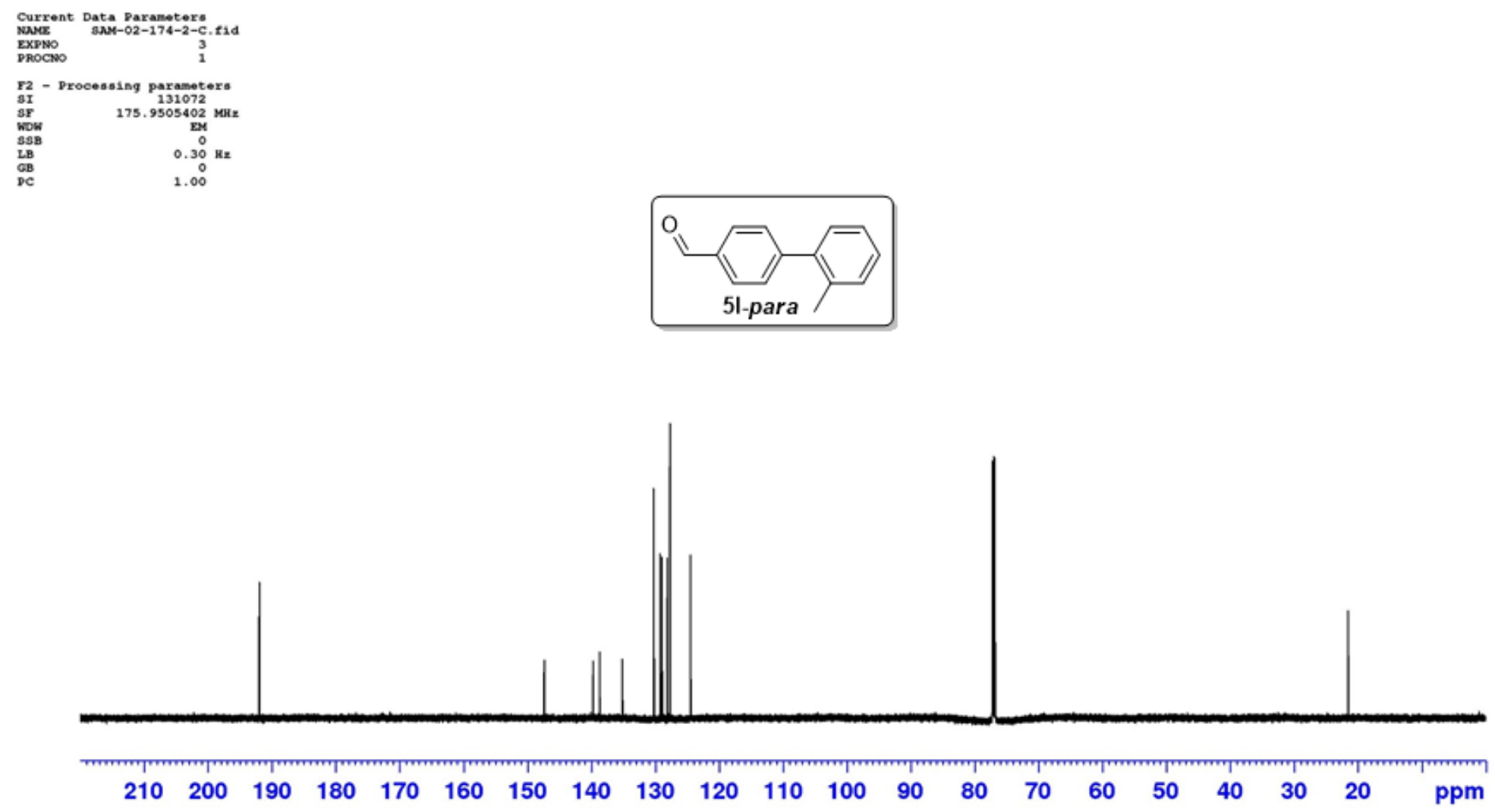
Solvent: $\mathrm{CDCl}_{3}$

SFO1: $700 \mathrm{MHz}$

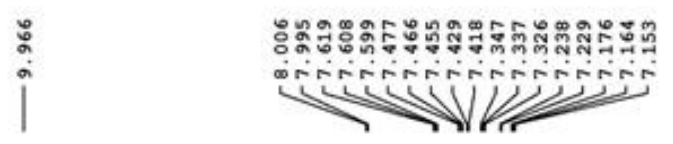

|

SAM-02-174-1

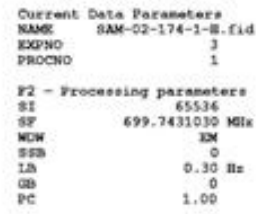

(1-ortho

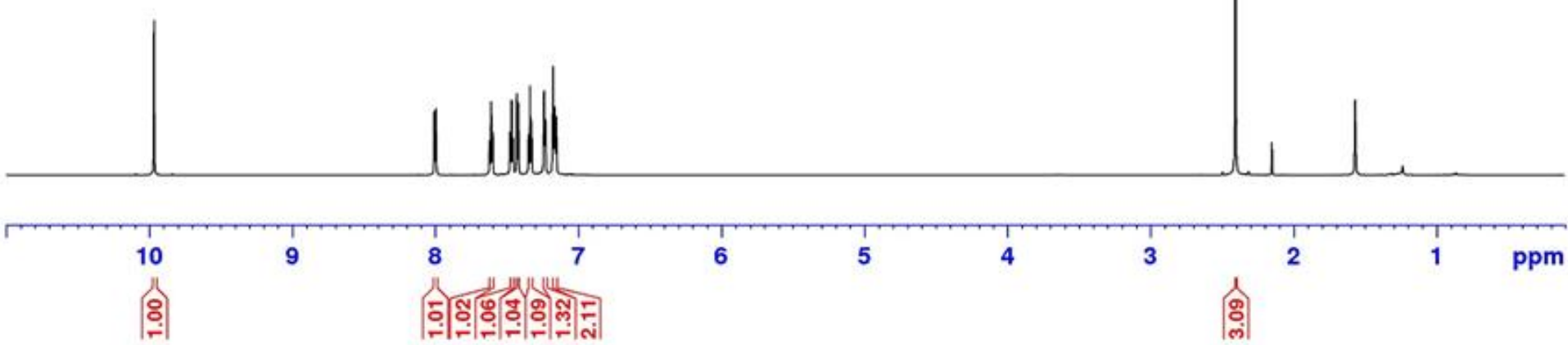


Solvent: $\mathrm{CDCl}_{3}$

SFO1: $175 \mathrm{MHz}$

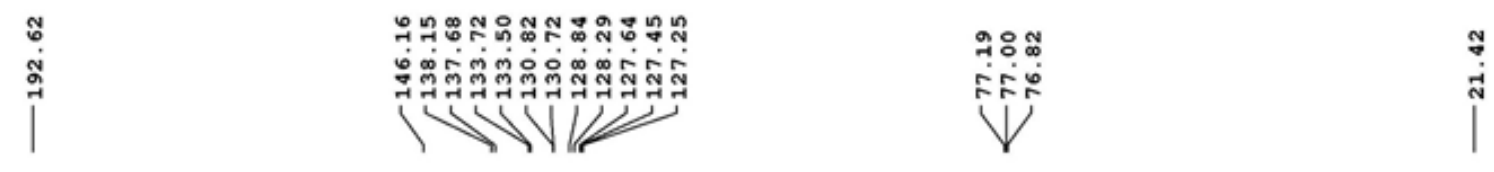

SAM-02-174-1
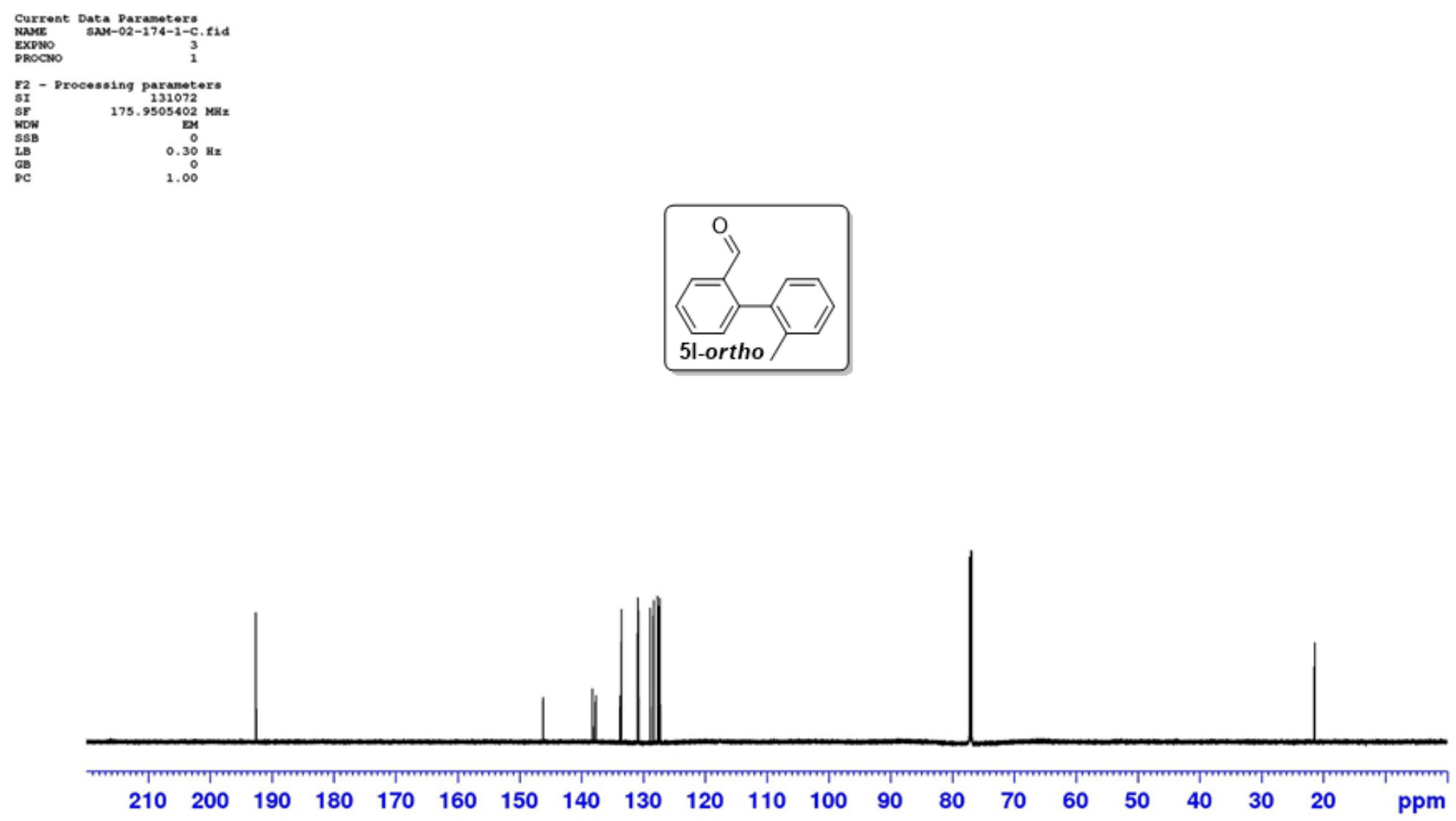
Solvent: $\mathrm{CDCl}_{3}$

SFO1: $700 \mathrm{MHz}$

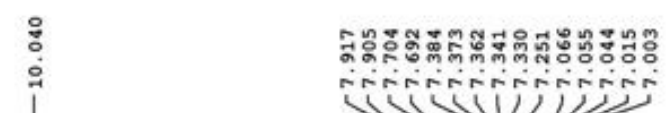

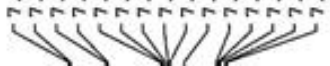

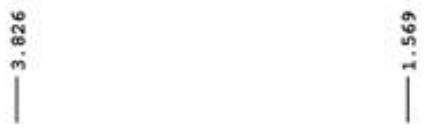

SAM-02-220-2

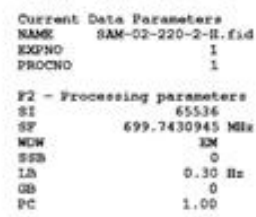
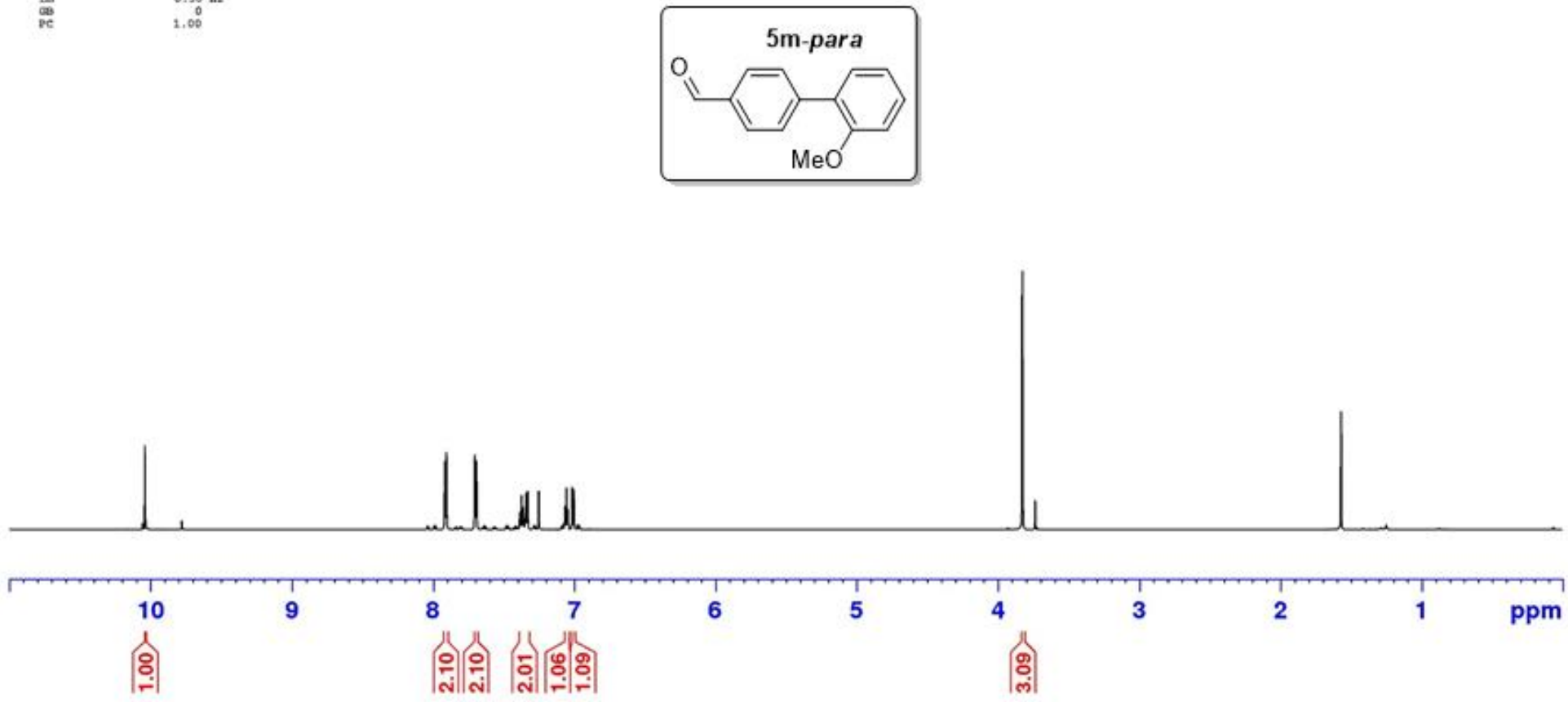
Solvent: $\mathrm{CDCl}_{3}$

SFO1: $175 \mathrm{MHz}$

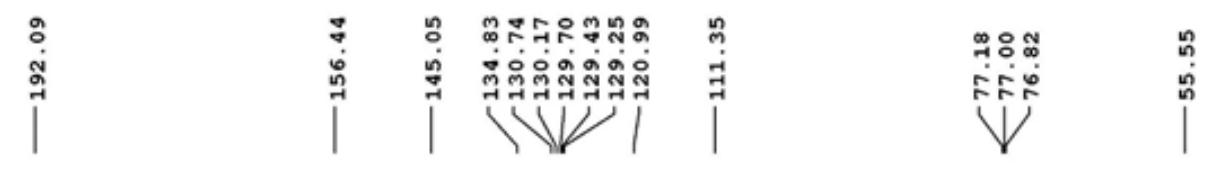

SAM-02-202-2

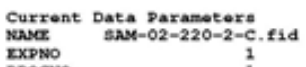

PRPOCNO
PRONO

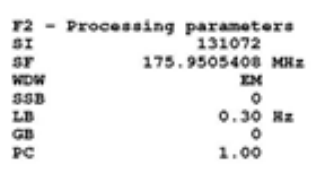
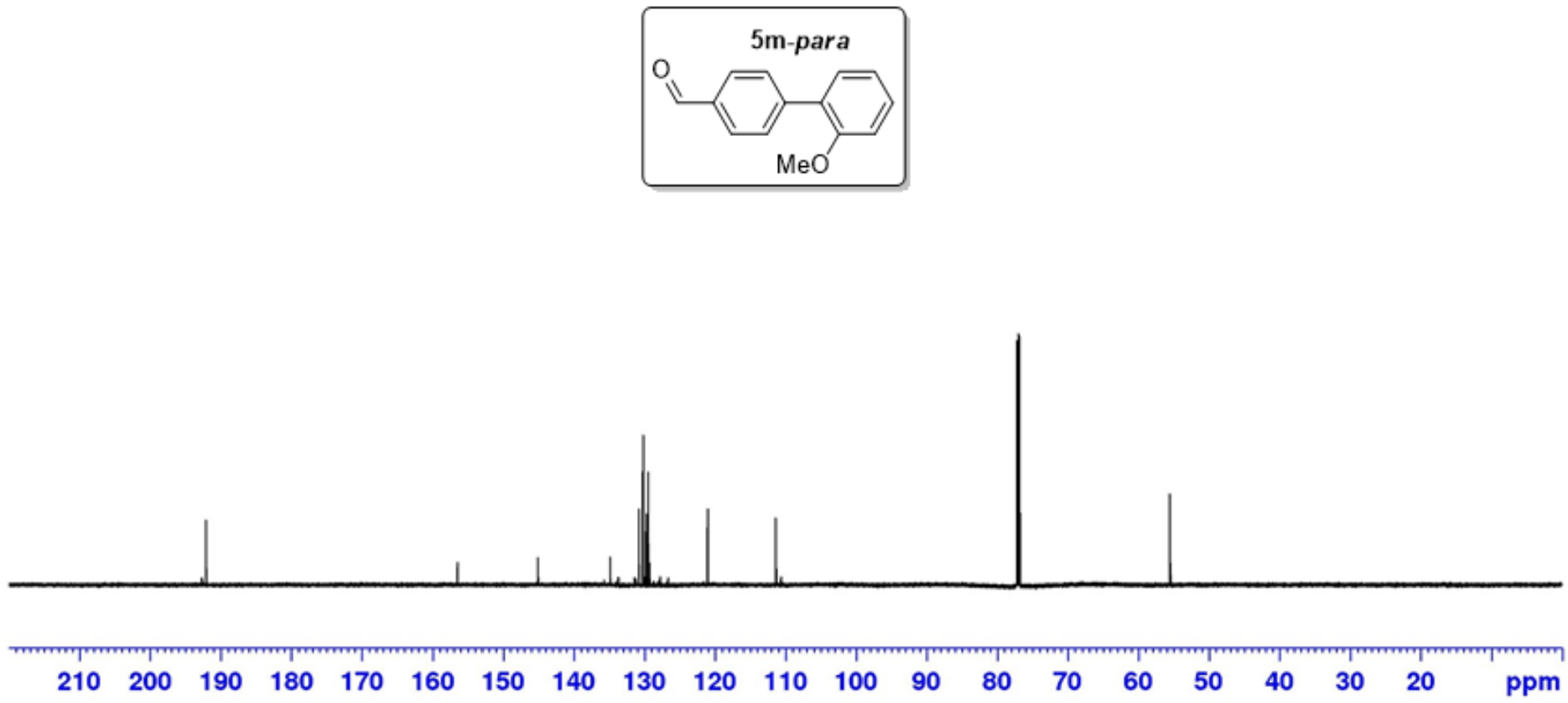
Solvent: $\mathrm{CDCl}_{3}$

SFO1: $700 \mathrm{MHz}$
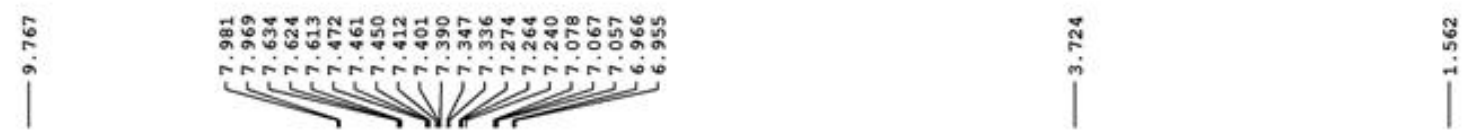

SAM-02-220-1

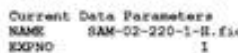

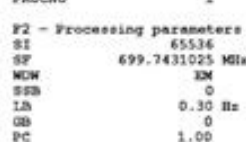

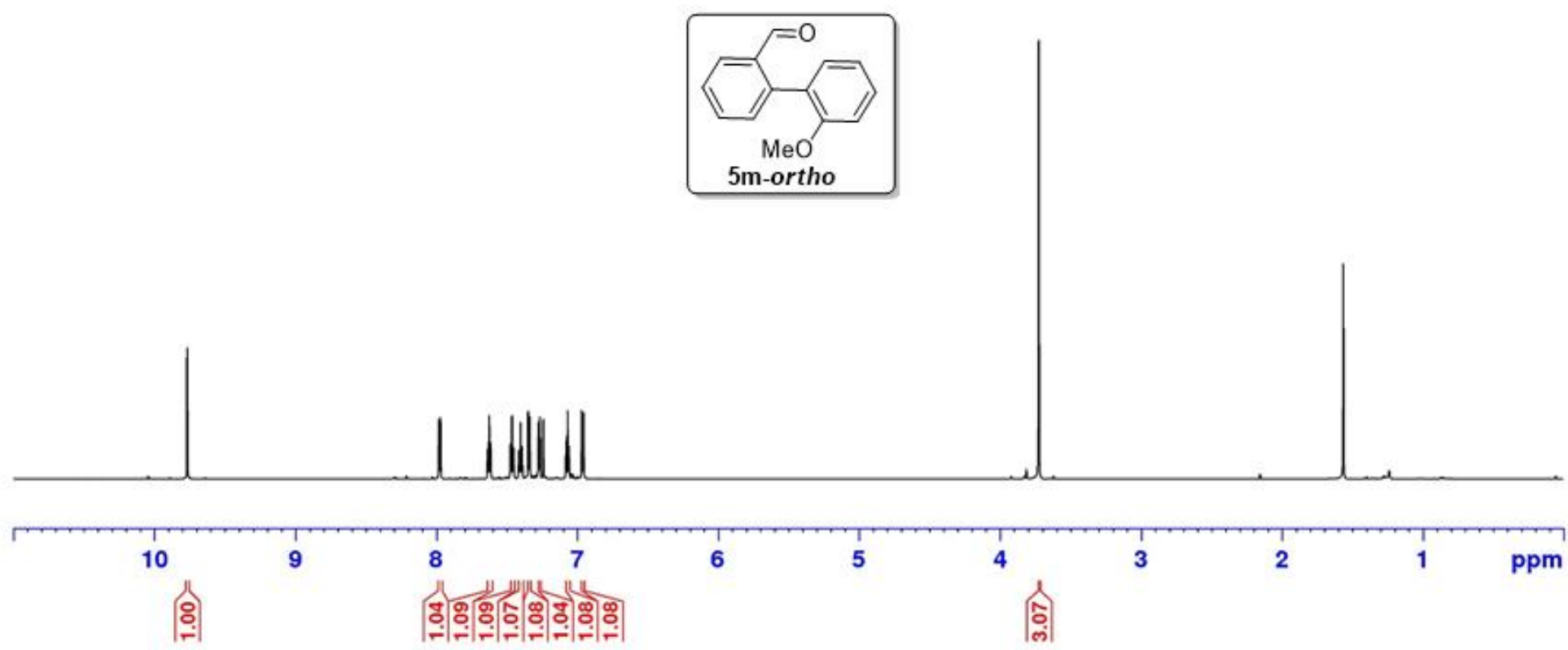


Solvent: $\mathrm{CDCl}_{3}$

SFO1: $175 \mathrm{MHz}$

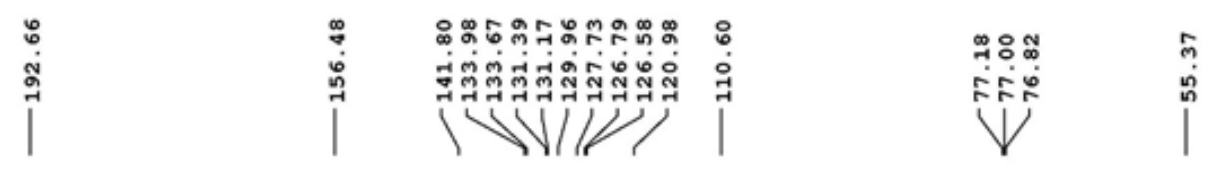

SAM-02-202-1
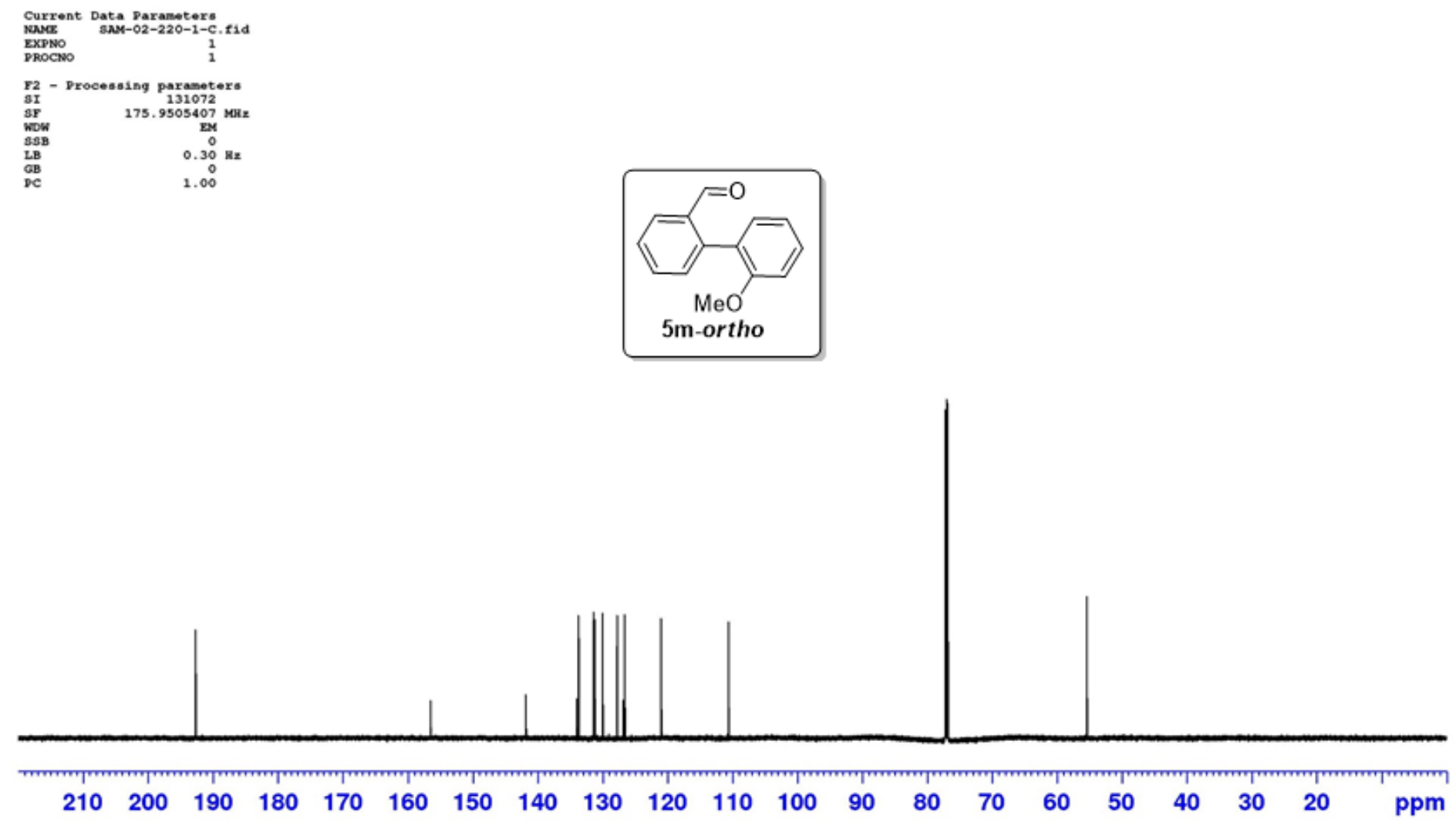
Solvent: $\mathrm{CDCl}_{3}$

SFO1: $700 \mathrm{MHz}$

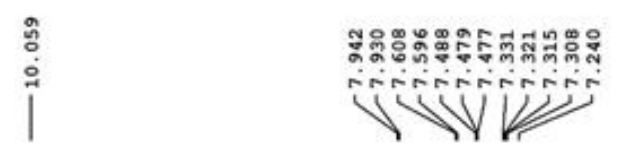

SAM-02-190-2

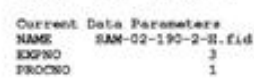

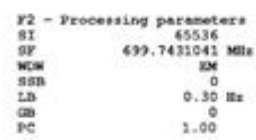
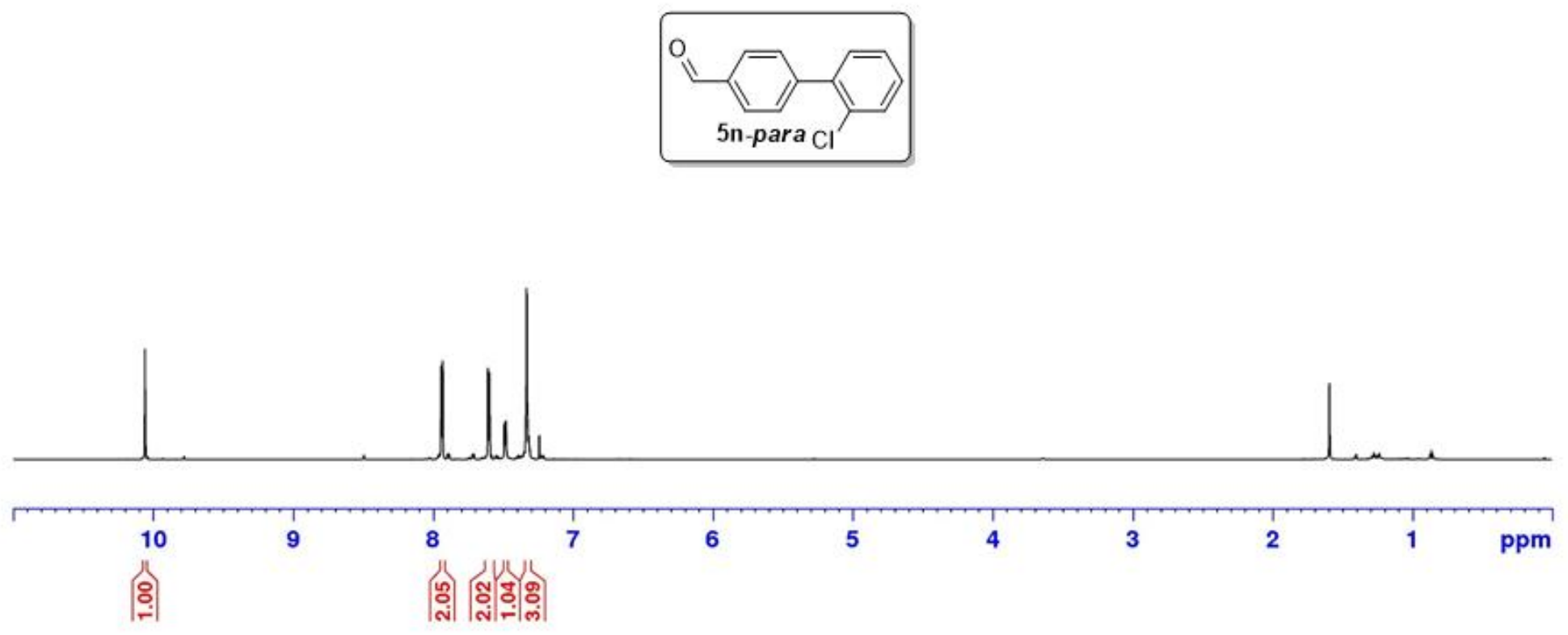
Solvent: $\mathrm{CDCl}_{3}$

SFO1: $175 \mathrm{MHz}$

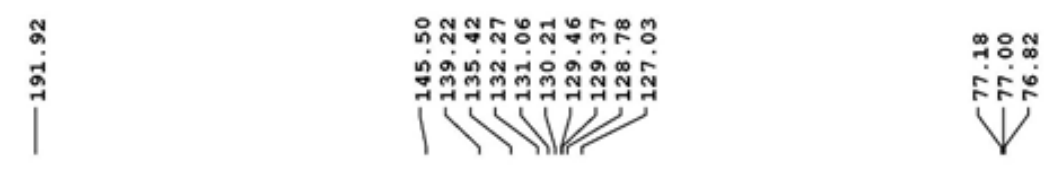

SAM-02-190-2
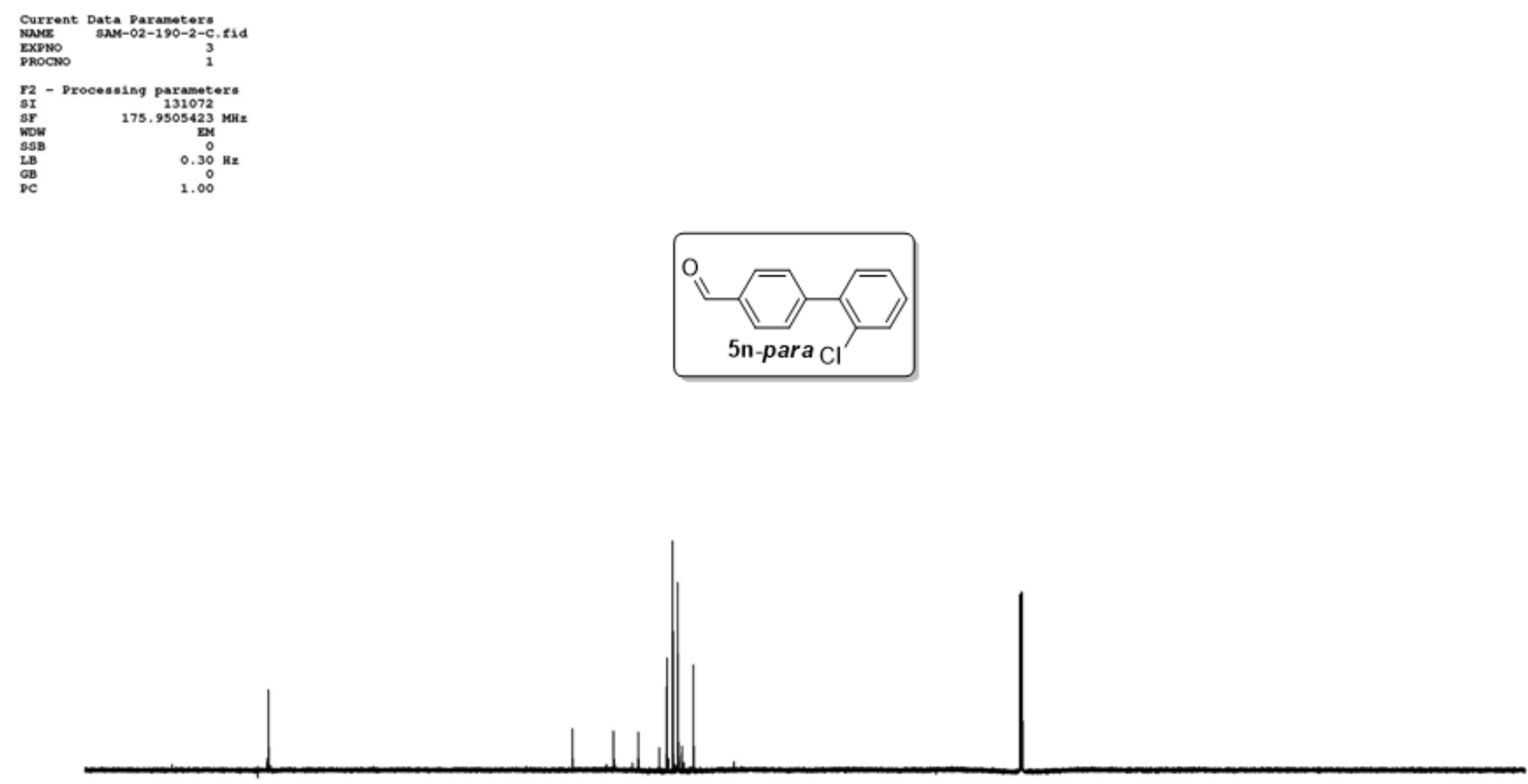

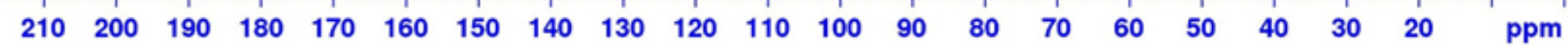


Solvent: $\mathrm{CDCl}_{3}$

SFO1: $700 \mathrm{MHz}$

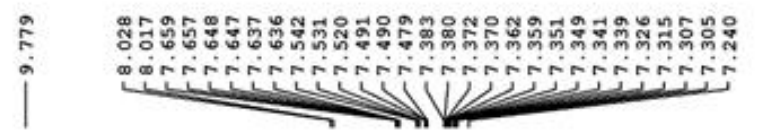

\section{SAM-02-190-1}

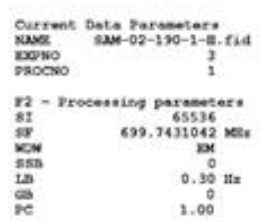

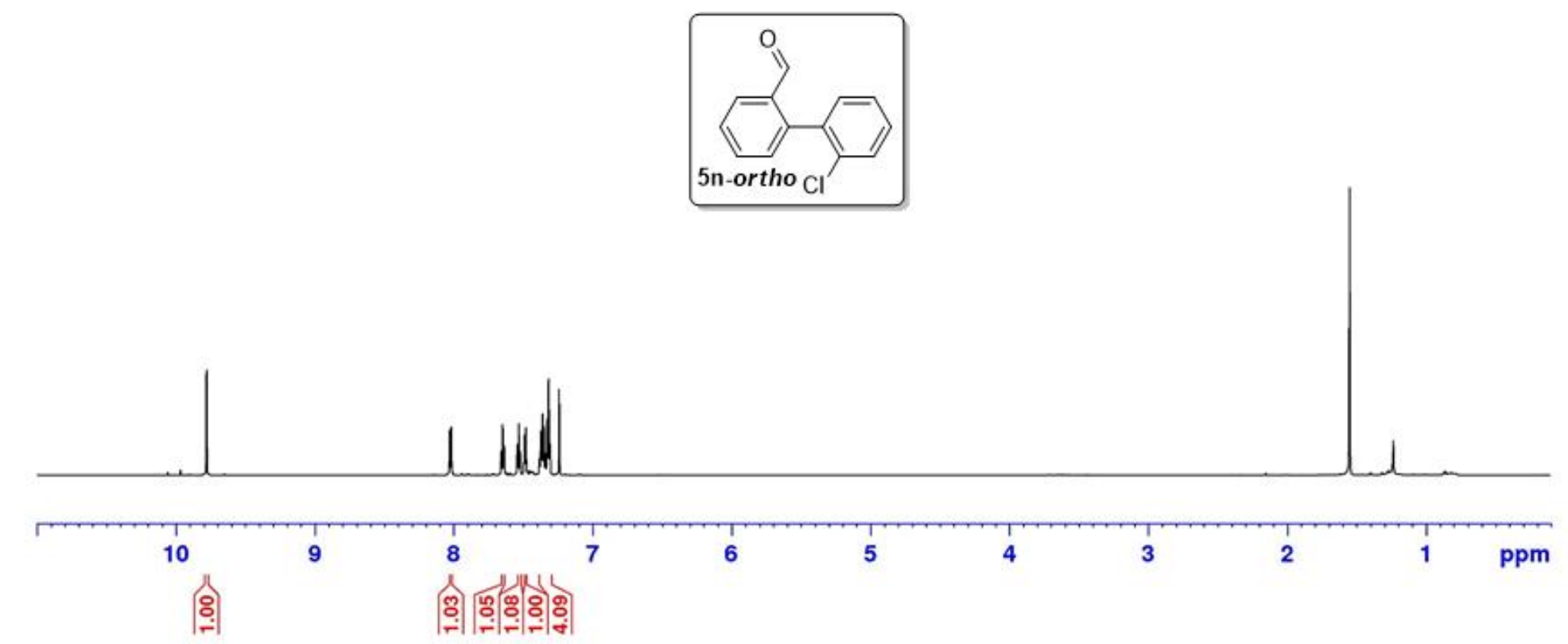


Solvent: $\mathrm{CDCl}_{3}$

SFO1: $175 \mathrm{MHz}$

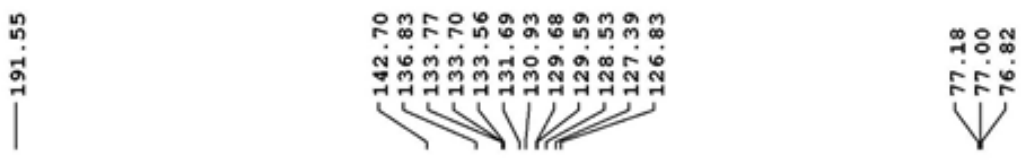

SAM-02-190-1
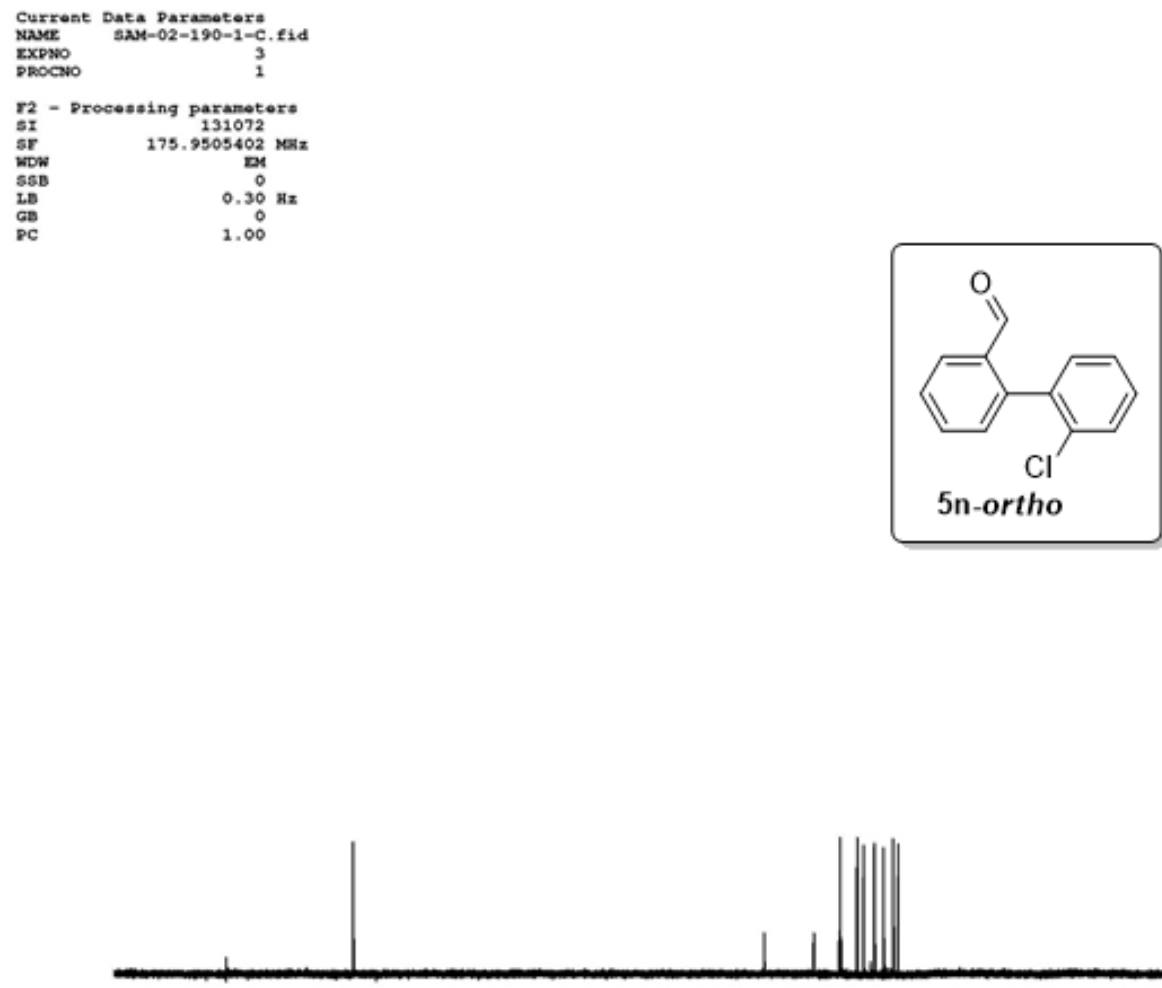

V

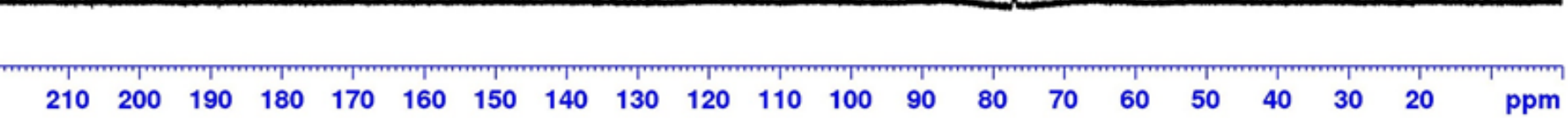


Solvent: $\mathrm{CDCl}_{3}$ SFO1: $700 \mathrm{MHz}$

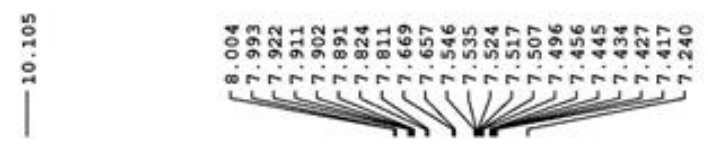

$\stackrel{5}{5}$

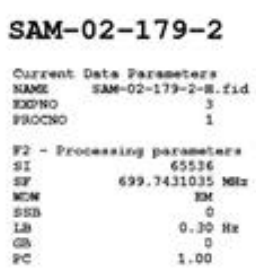
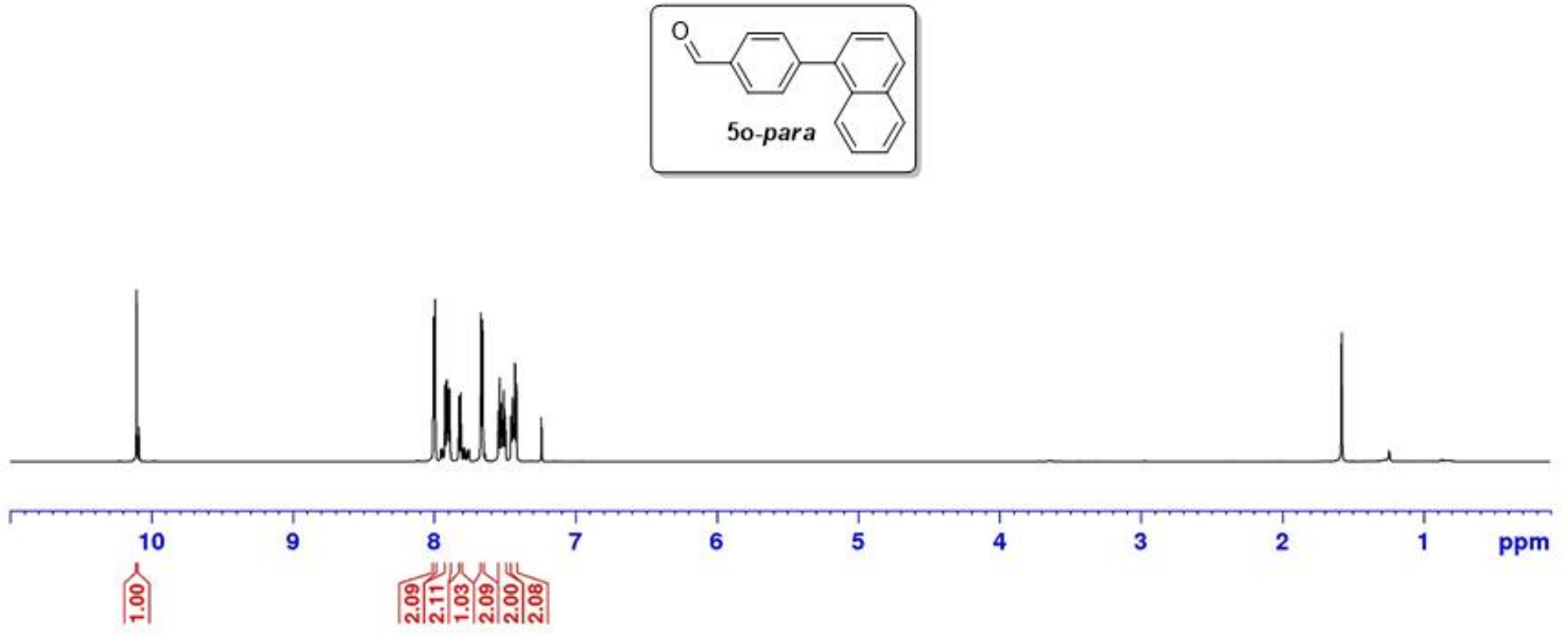
Solvent: $\mathrm{CDCl}_{3}$

SFO1: $175 \mathrm{MHz}$

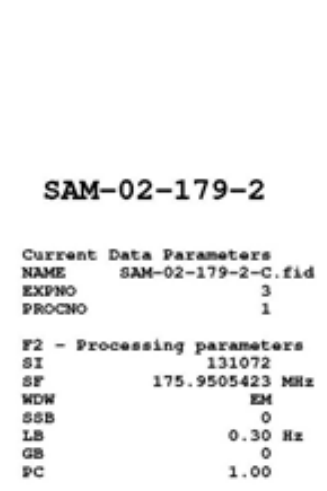

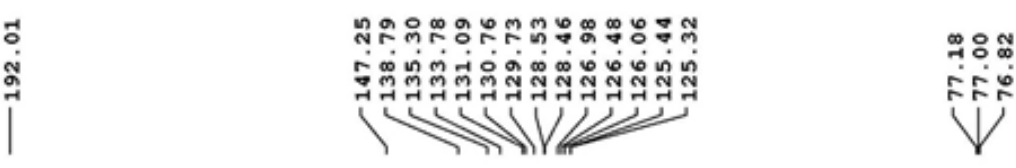

๓๐ํำ

ㄷํ
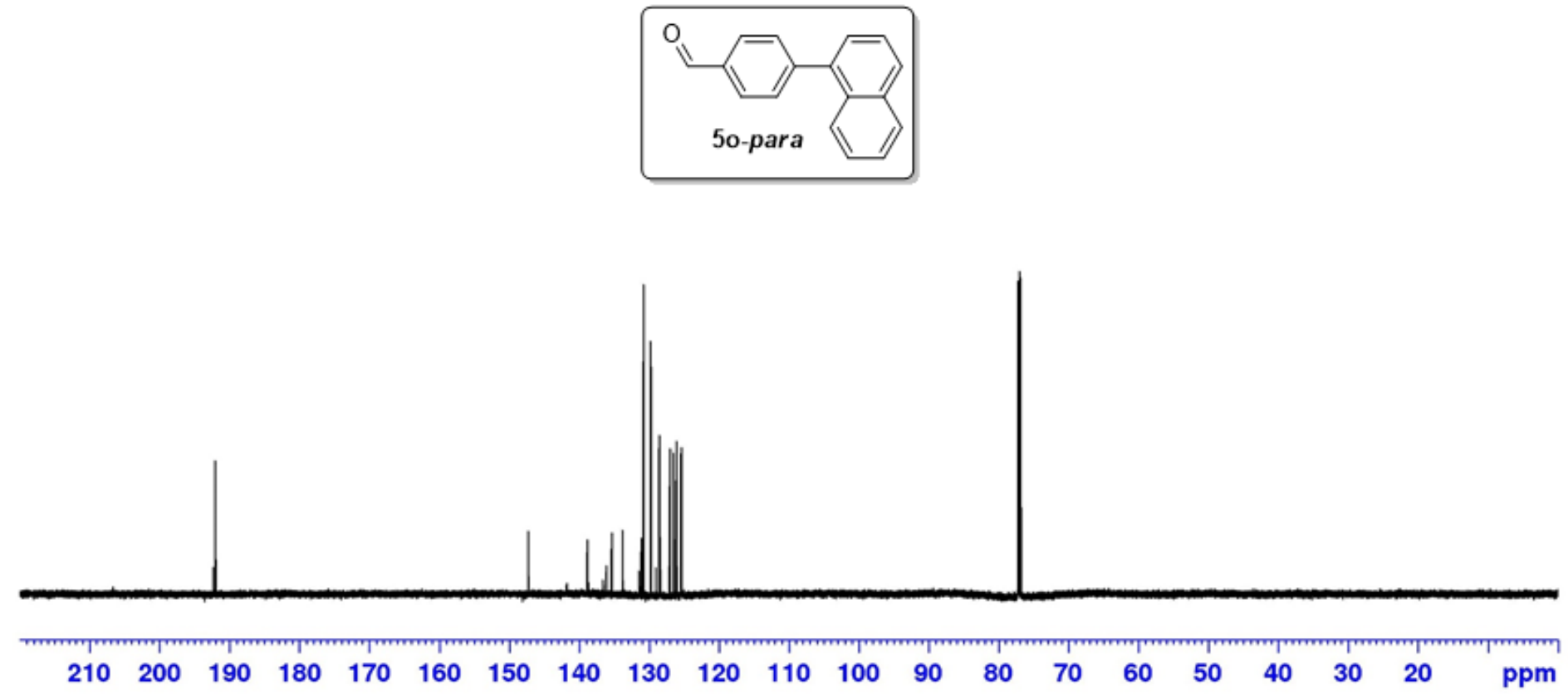
Solvent: $\mathrm{CDCl}_{3}$

SFO1: $700 \mathrm{MHz}$

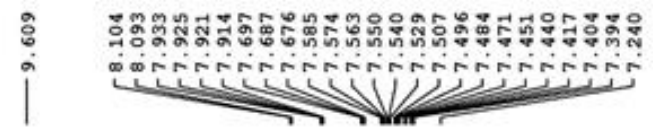

SAM-02-179-1

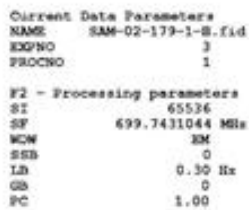
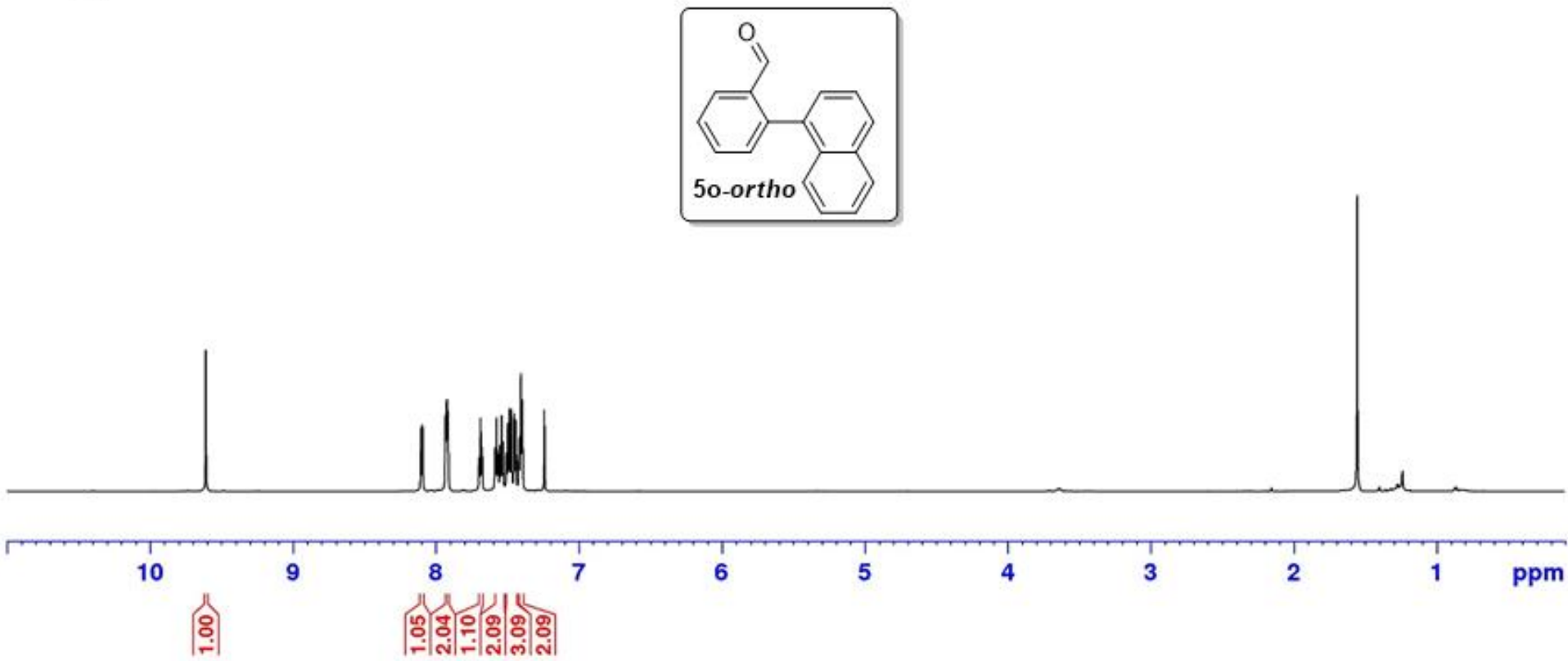
Solvent: $\mathrm{CDCl}_{3}$

SFO1: $175 \mathrm{MHz}$

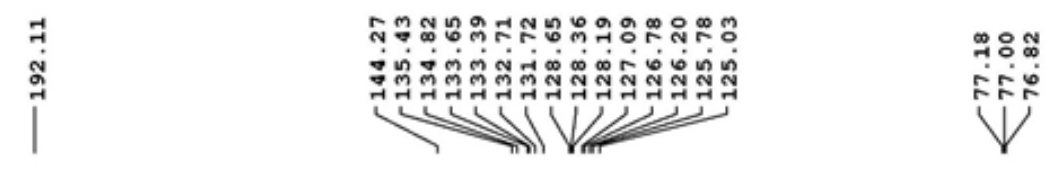

SAM-02-179-1
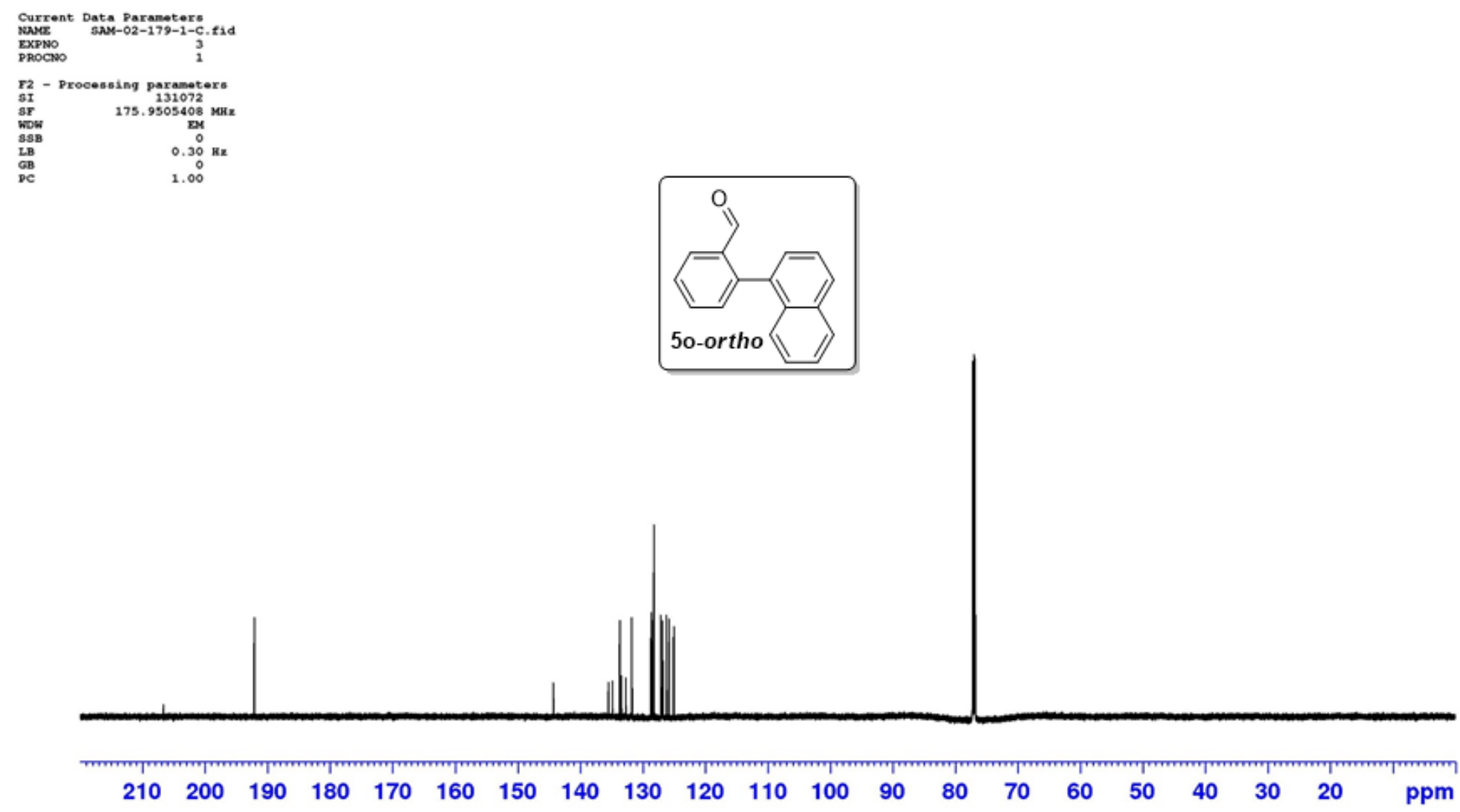

V 
Solvent: $\mathrm{CDCl}_{3}$

SFO1: $700 \mathrm{MHz}$

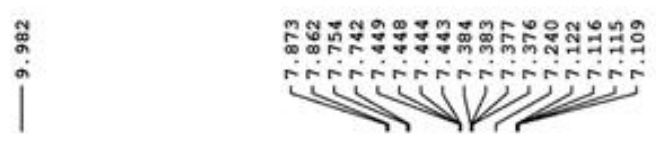

$$
\text { iิ }
$$

SAM-02-201-2

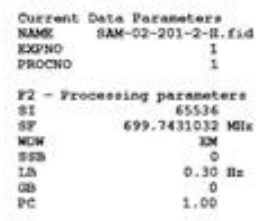
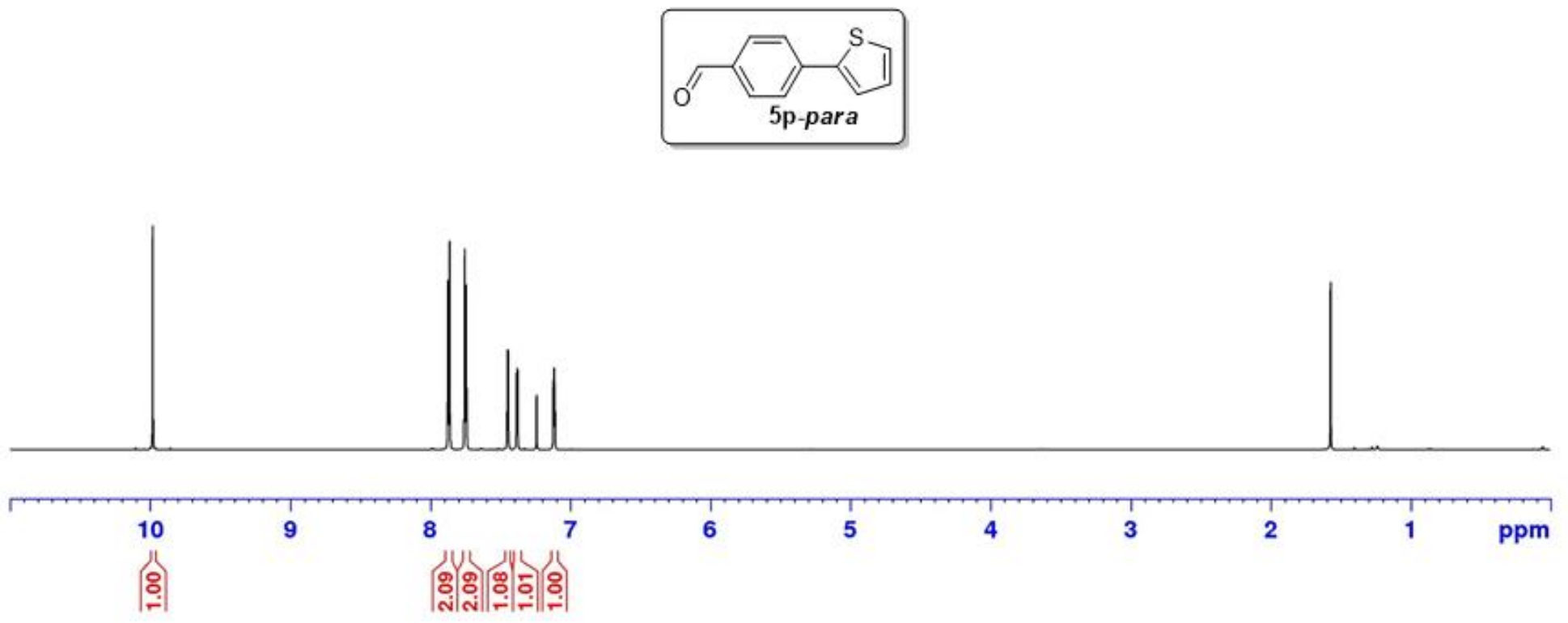
Solvent: $\mathrm{CDCl}_{3}$

SFO1: $175 \mathrm{MHz}$

$$
\text { 1) }
$$

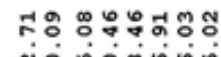

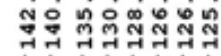

II $111 \mathrm{~K}$

$\underset{-\infty}{\infty}$

FF

SAM-02-201-2

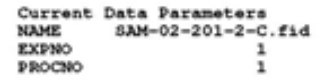

$$
\begin{aligned}
& 72 \text { - Processing paranotors }
\end{aligned}
$$

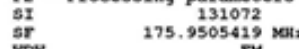

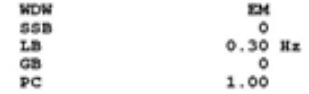
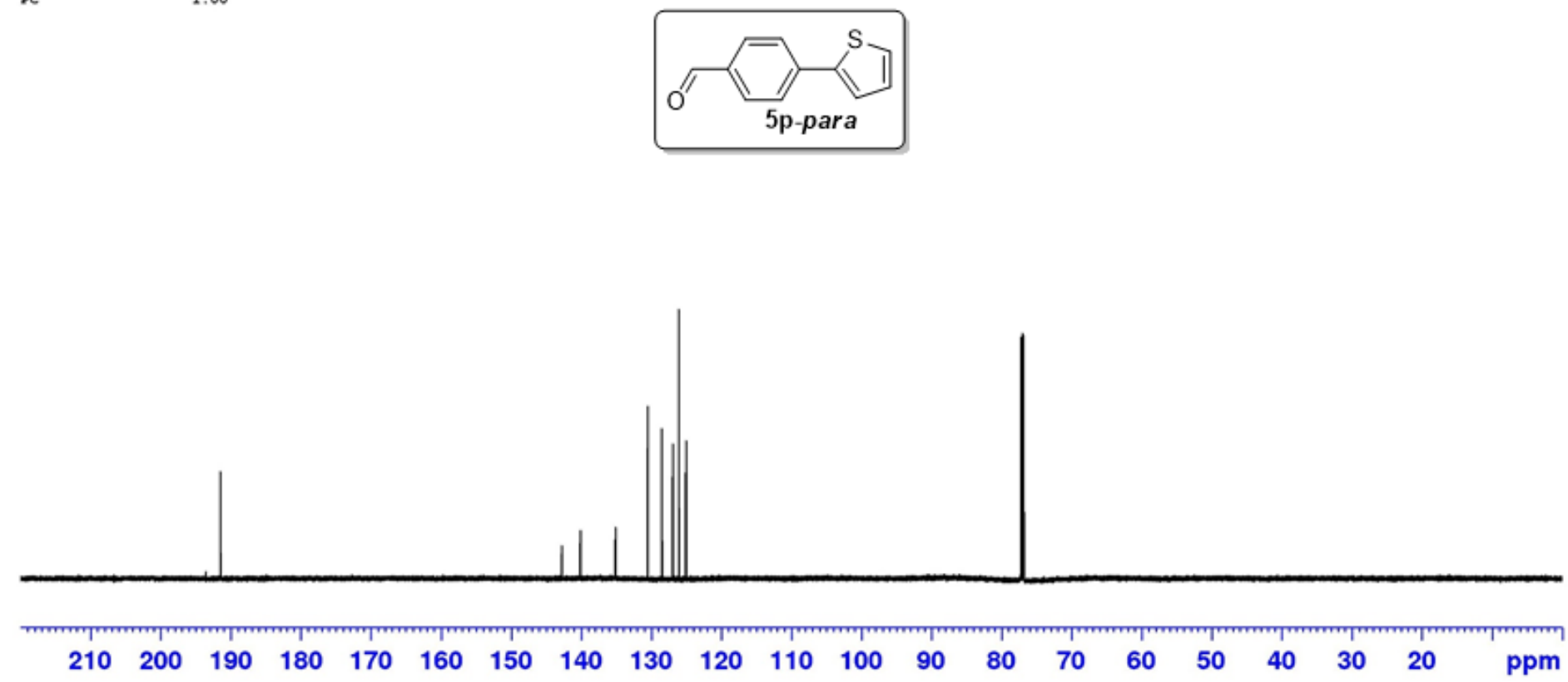
Solvent: $\mathrm{CDCl}_{3}$ SFO1: $700 \mathrm{MHz}$

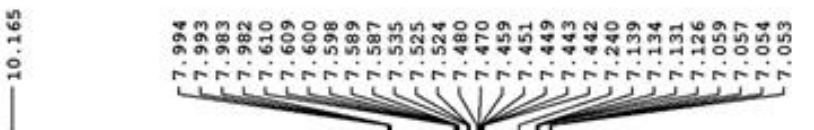

SAM-02-201-1

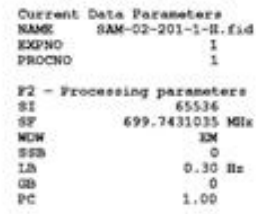
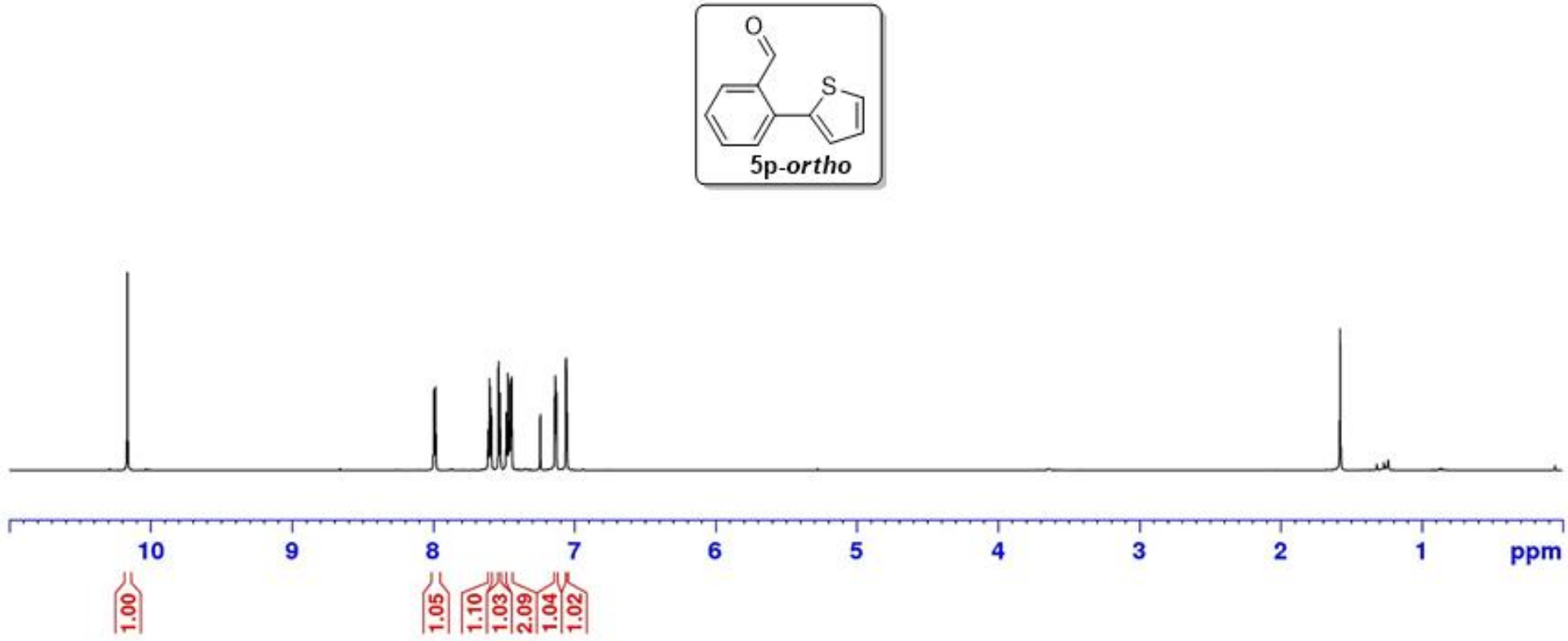
Solvent: $\mathrm{CDCl}_{3}$

SFO1: $175 \mathrm{MHz}$

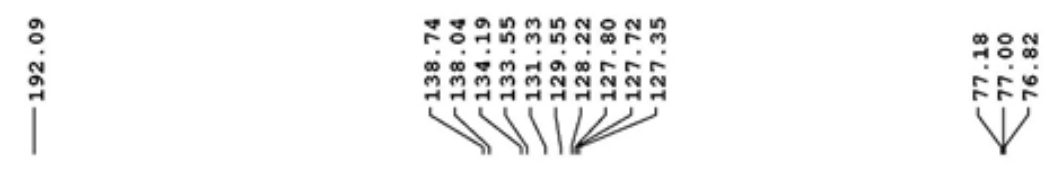

SAM-02-201-1
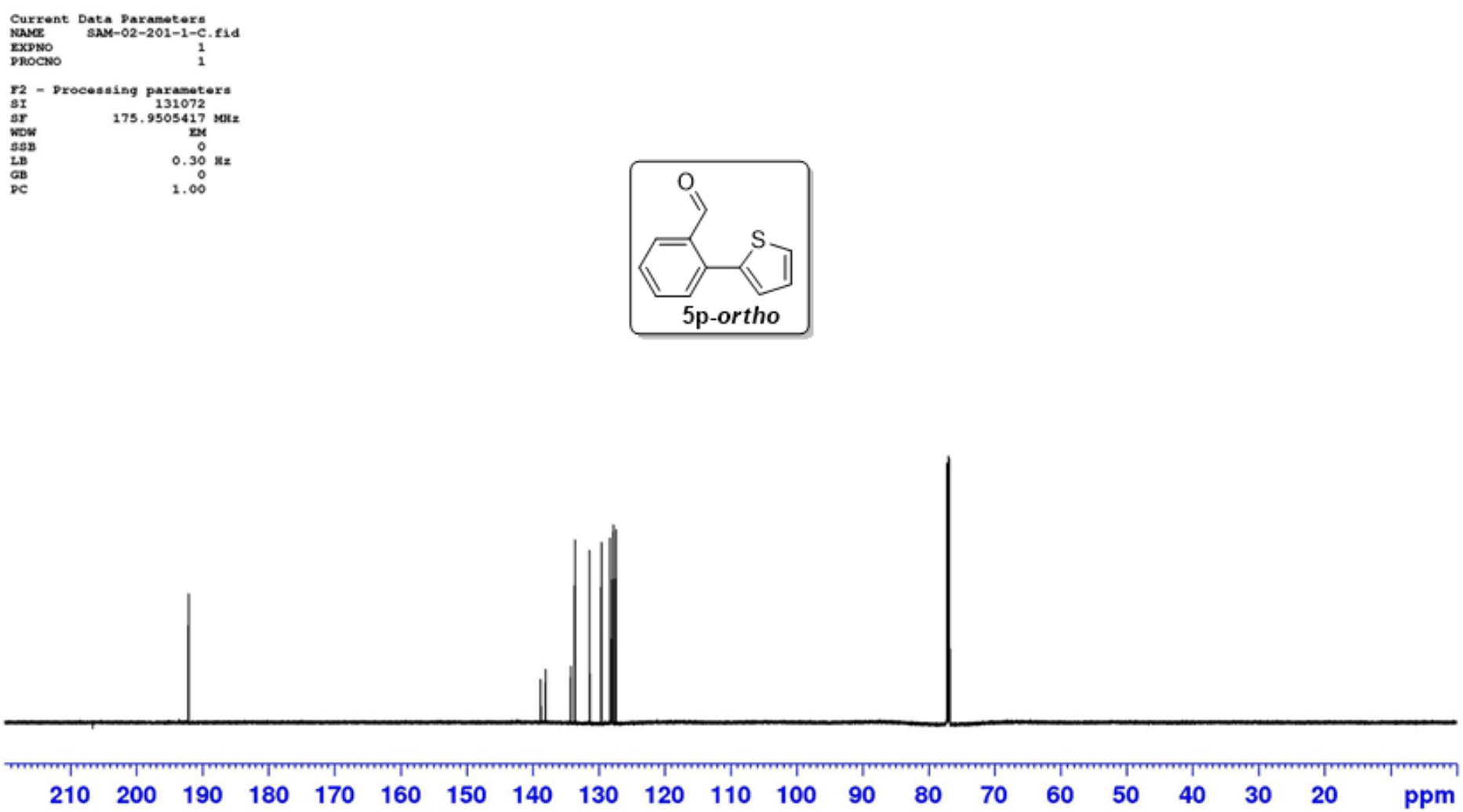
Solvent: $\mathrm{CDCl}_{3}$

SFO1: $700 \mathrm{MHz}$
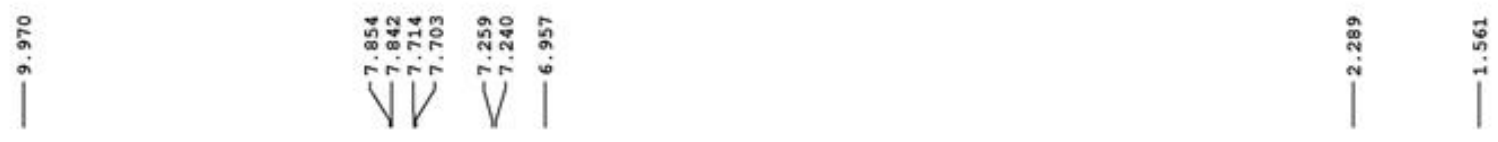

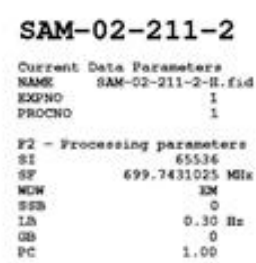

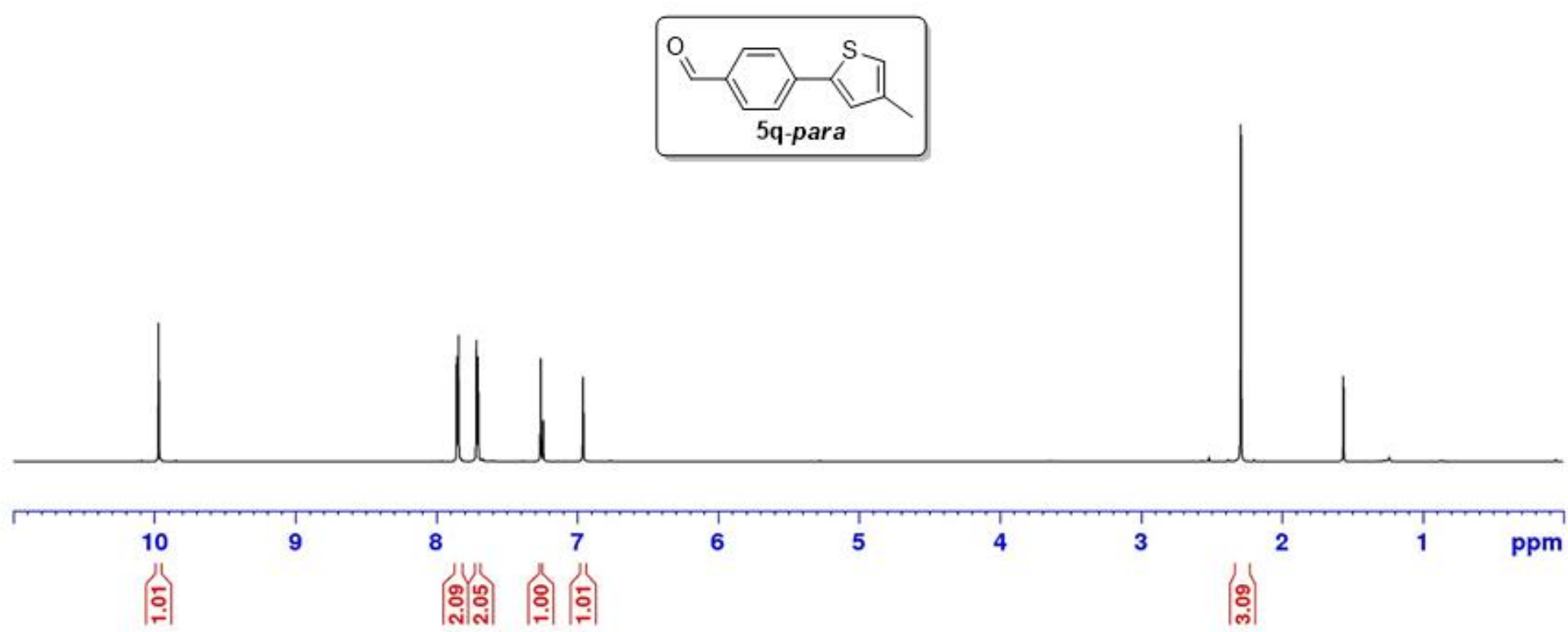


Solvent: $\mathrm{CDCl}_{3}$

SFO1: $175 \mathrm{MHz}$

$$
\text { 1) }
$$

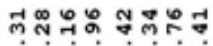

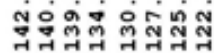

$1 / 111$

ㄸํㅇำ

FF

$\stackrel{\infty}{\oplus}$

SAM-02-211-2
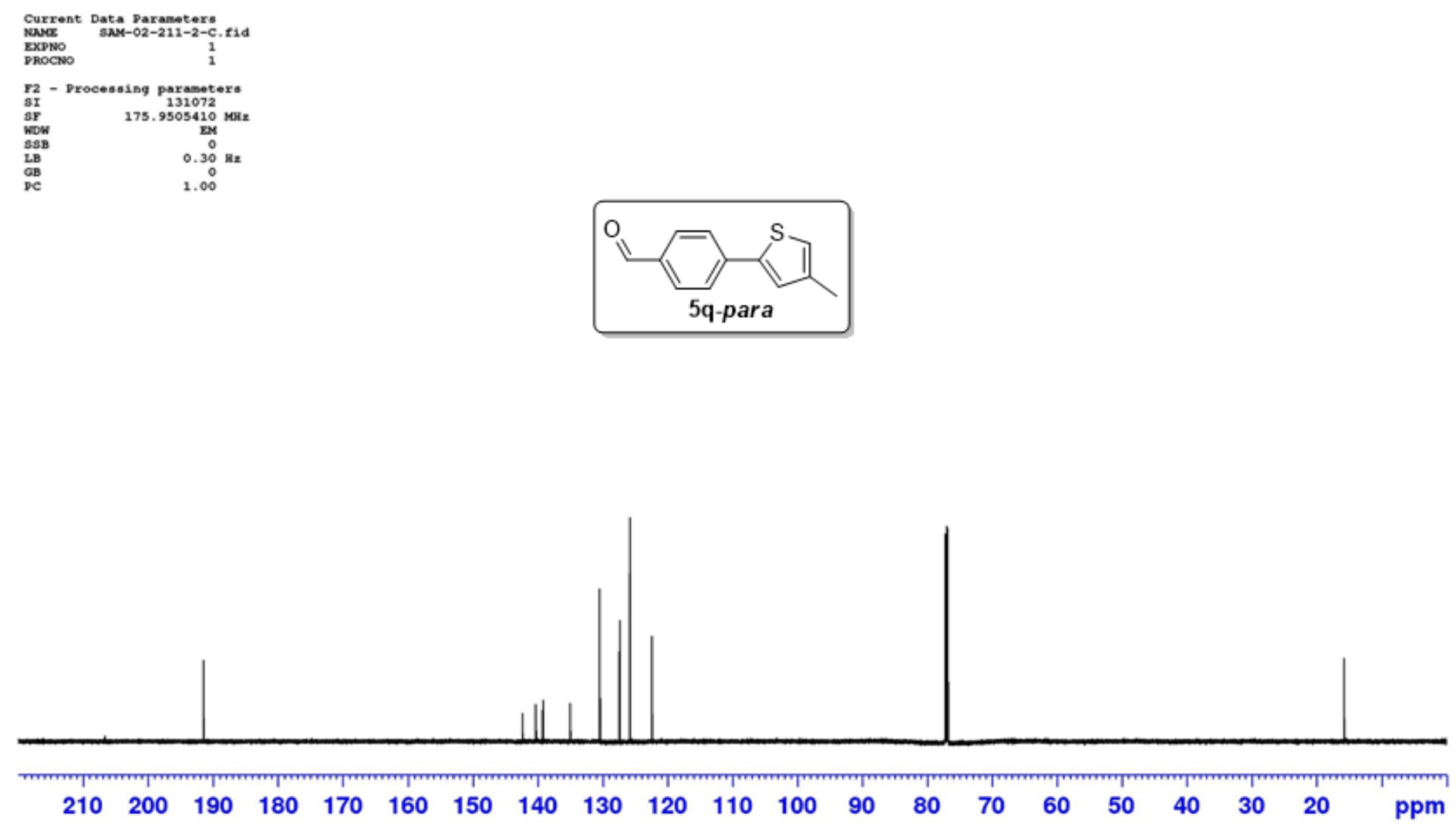
Solvent: $\mathrm{CDCl}_{3}$ SFO1: $700 \mathrm{MHz}$

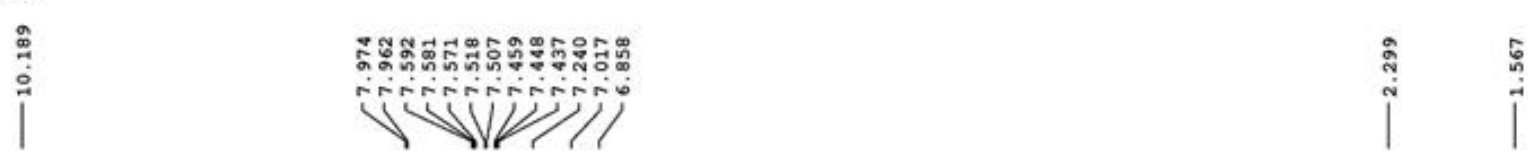

SAM-02-211-1

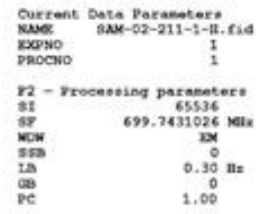

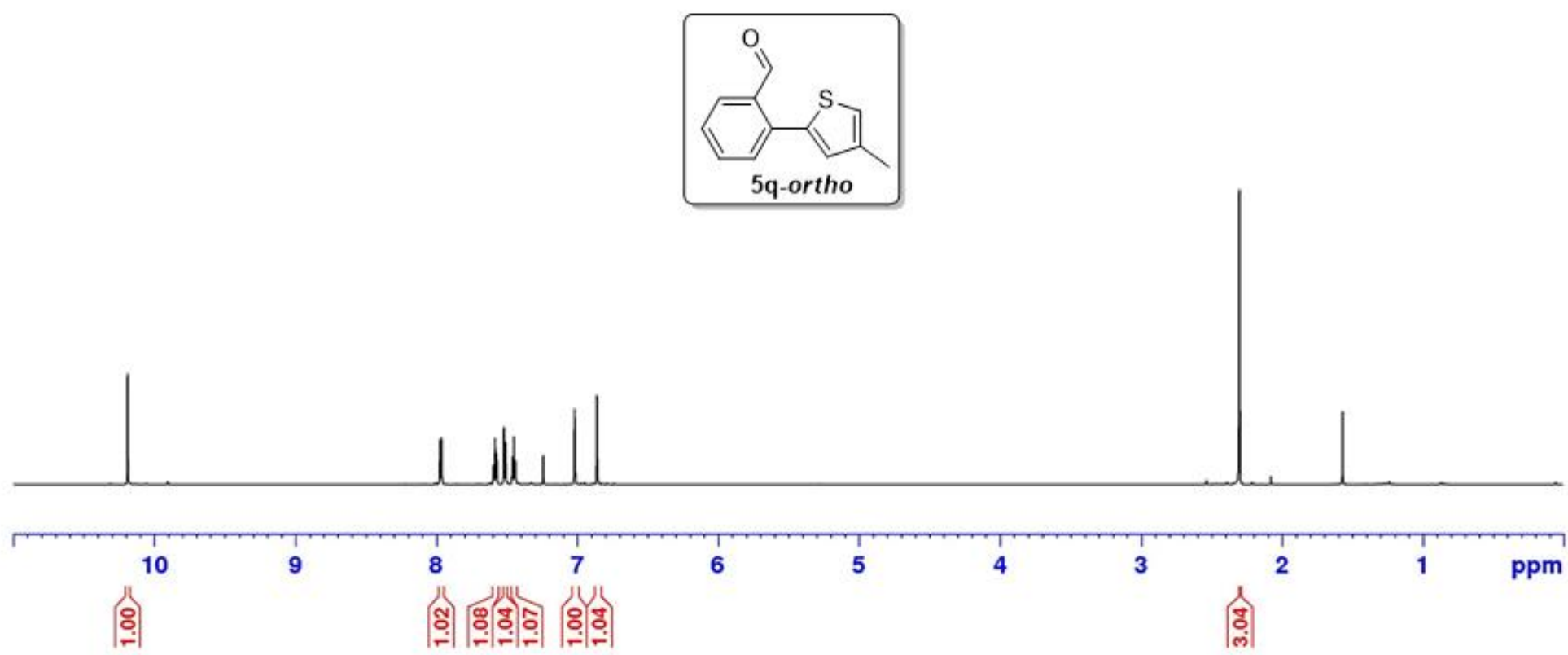


Solvent: $\mathrm{CDCl}_{3}$

SFO1: $175 \mathrm{MHz}$

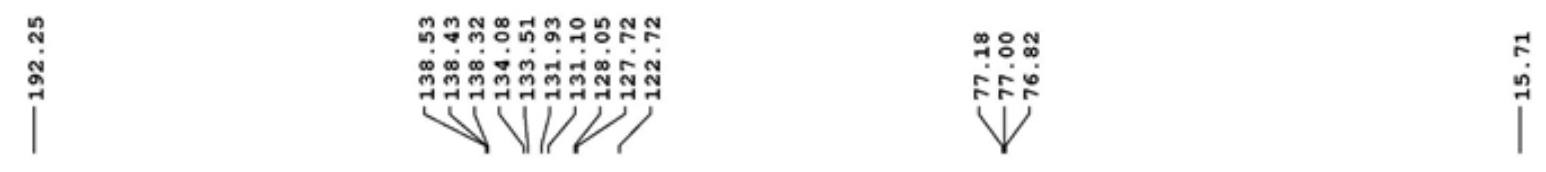

SAM-02-211-1
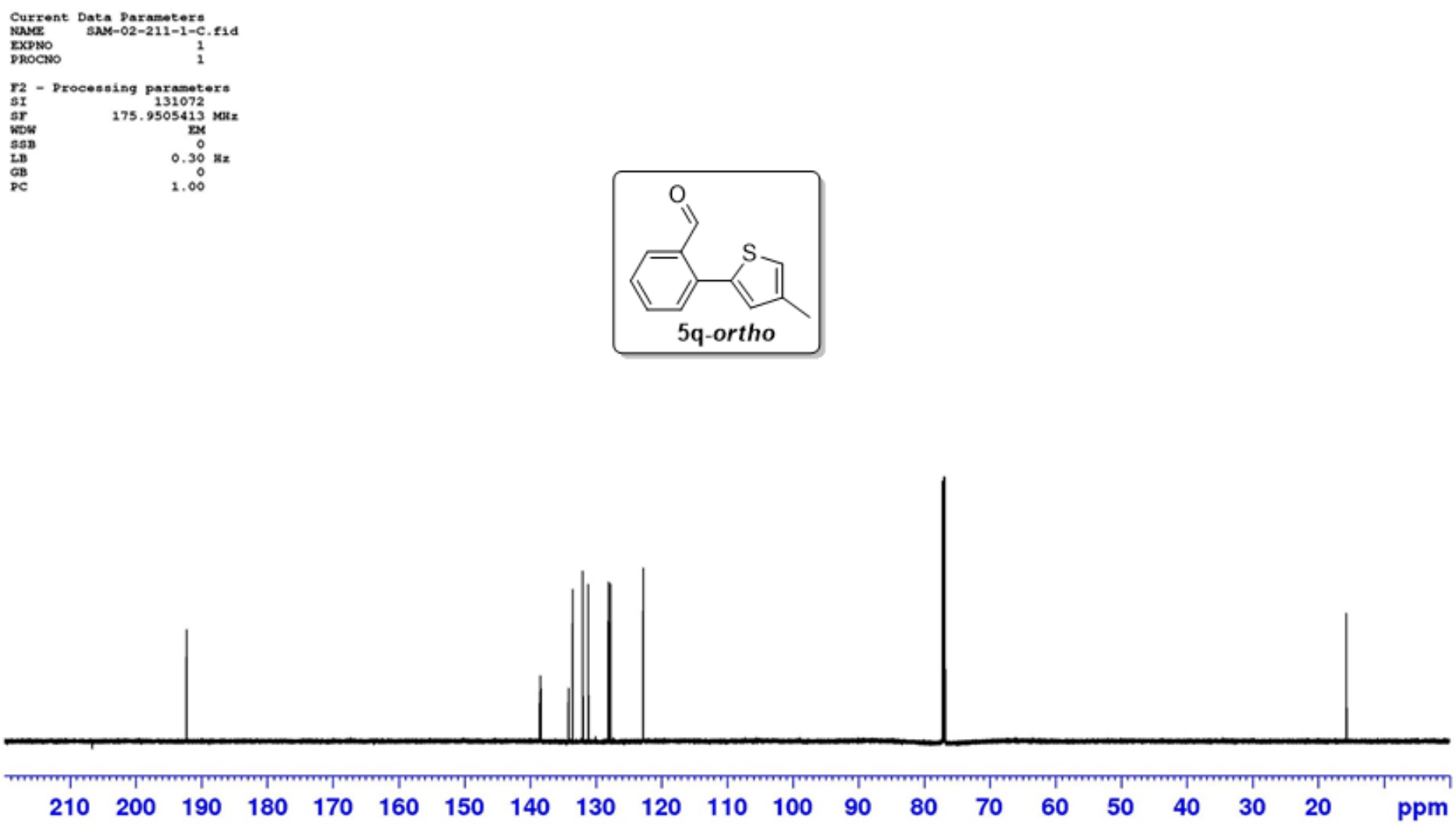
Solvent: $\mathrm{CDCl}_{3}$

SFO1: $700 \mathrm{MHz}$

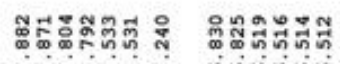

$\tilde{V} \hat{V} \hat{V} \hat{i} \dot{V} \Psi$

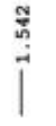

SAM-02-204-2

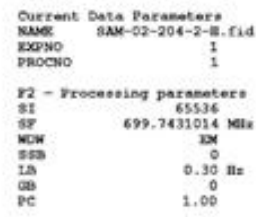
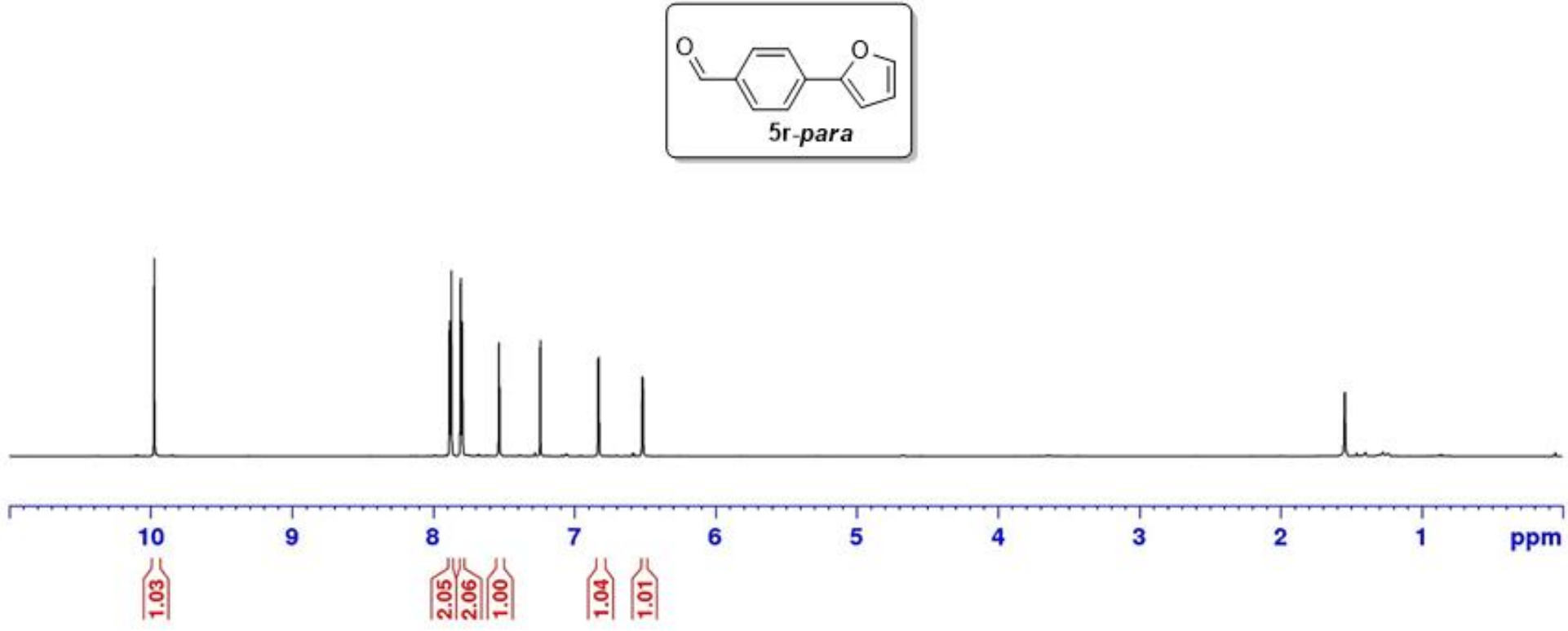
Solvent: $\mathrm{CDCl}_{3}$

SFO1: $175 \mathrm{MHz}$

|
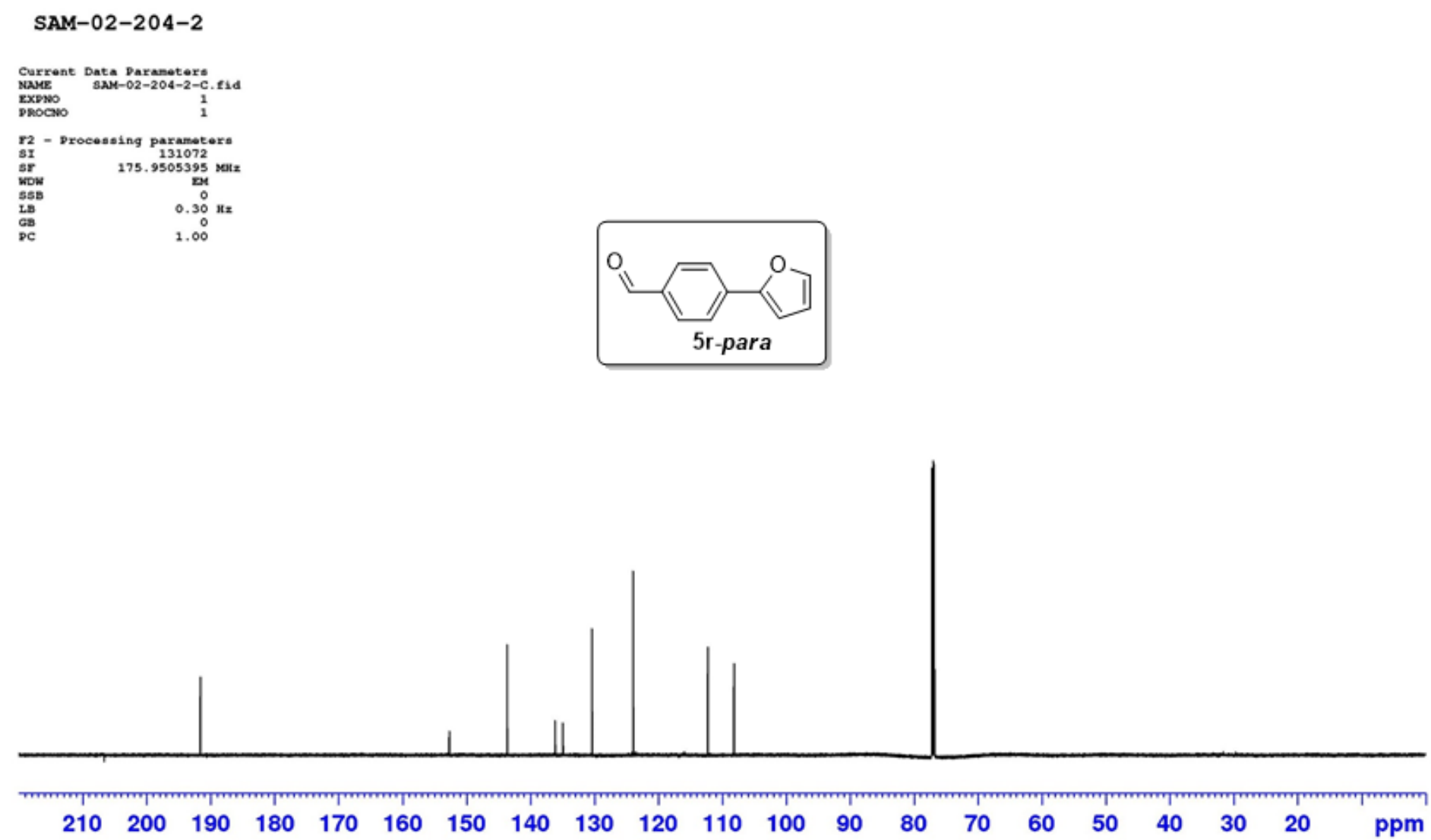
Solvent: $\mathrm{CDCl}_{3}$

SFO1: $700 \mathrm{MHz}$

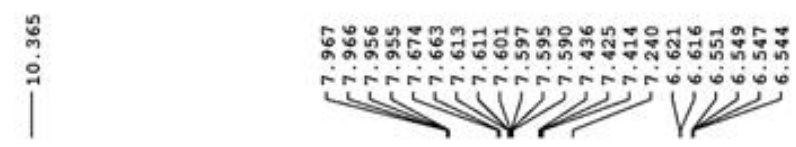

SAM-02-204-1

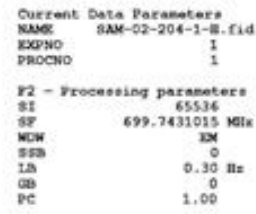
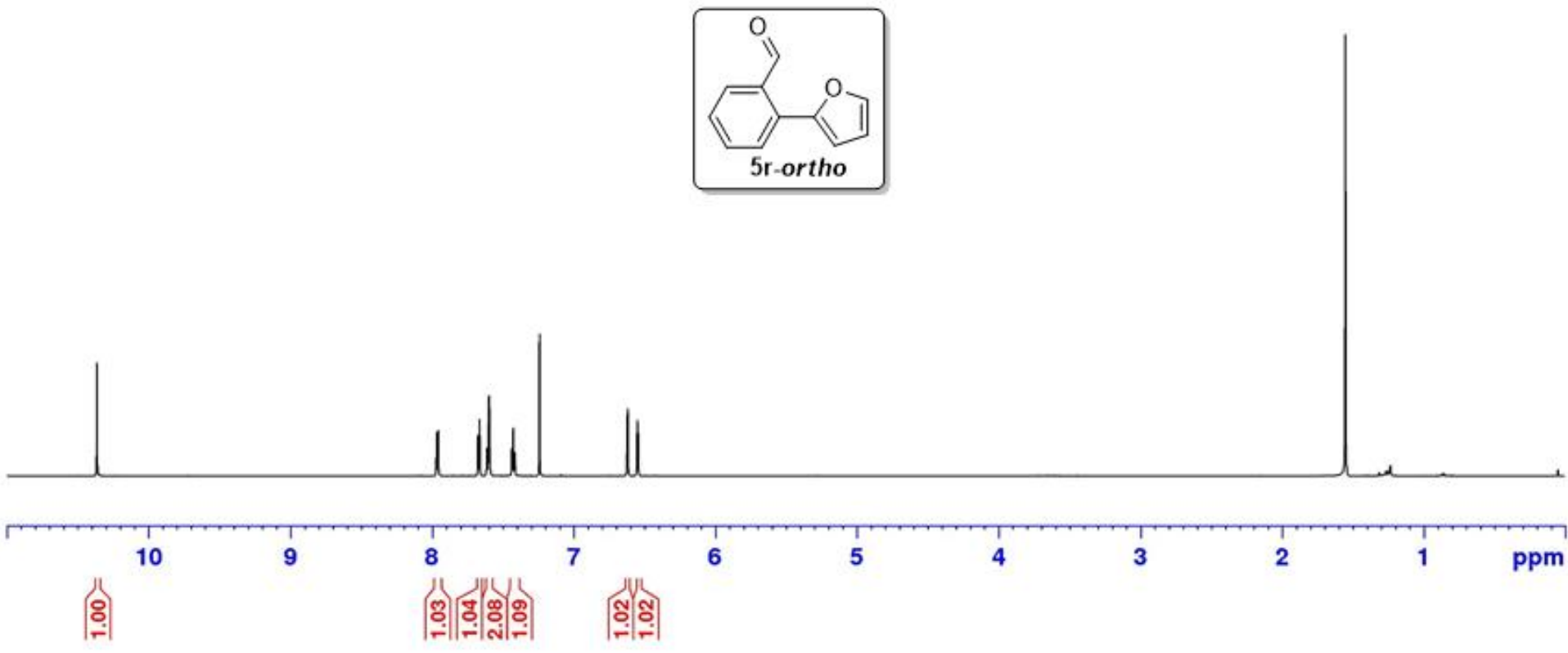
Solvent: $\mathrm{CDCl}_{3}$

SFO1: $175 \mathrm{MHz}$

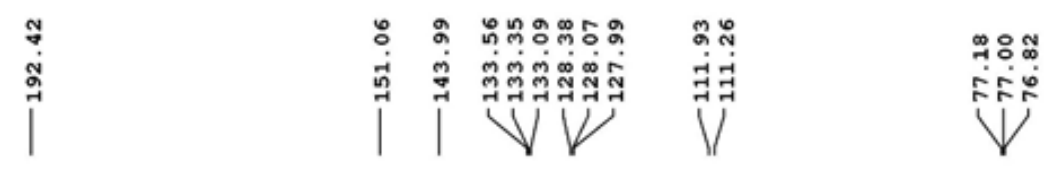

SAM-02-204-1
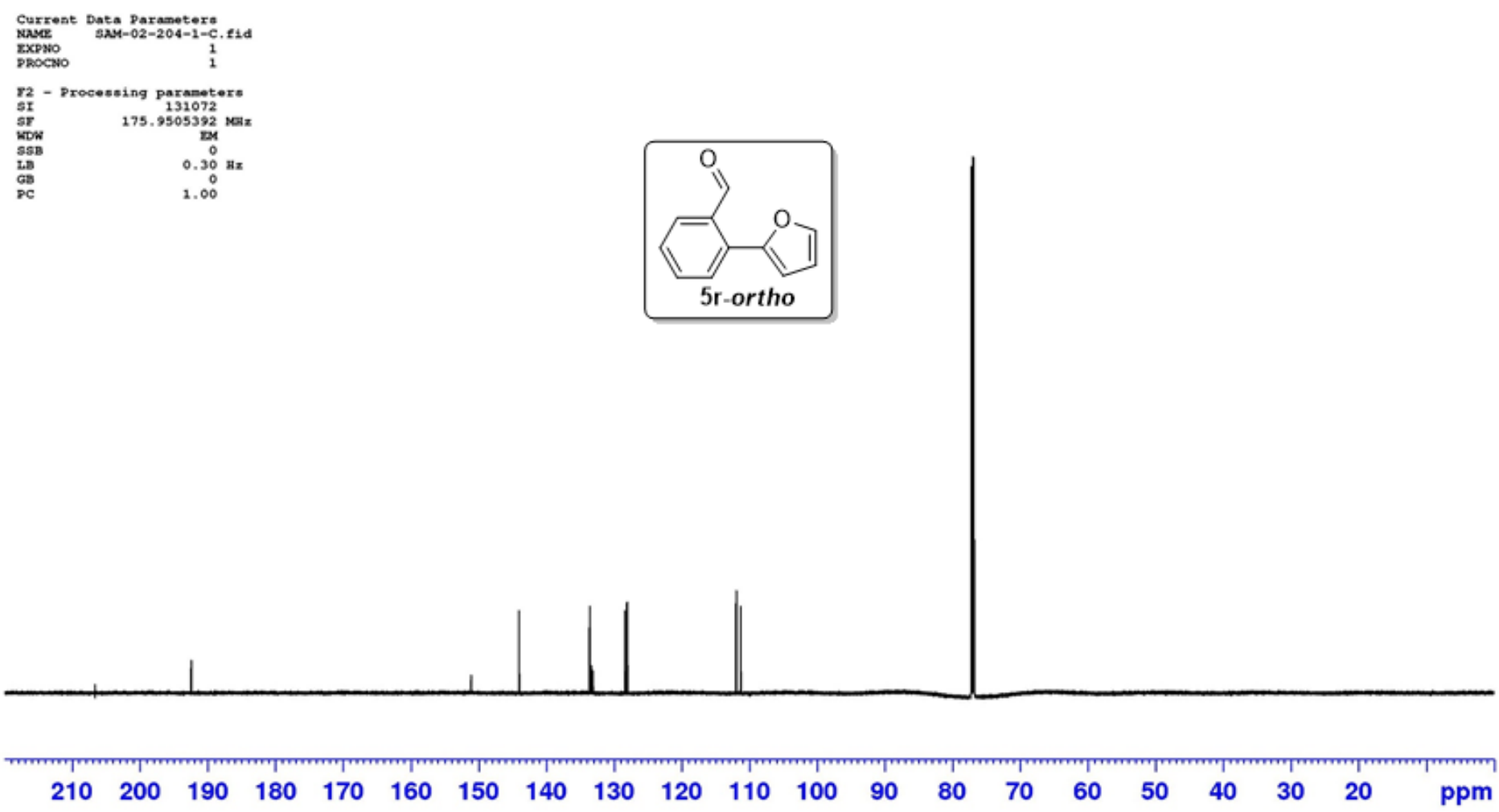
Solvent: $\mathrm{CDCl}_{3}$

SFO1: $400 \mathrm{MHz}$

|

SAM-02-228-3

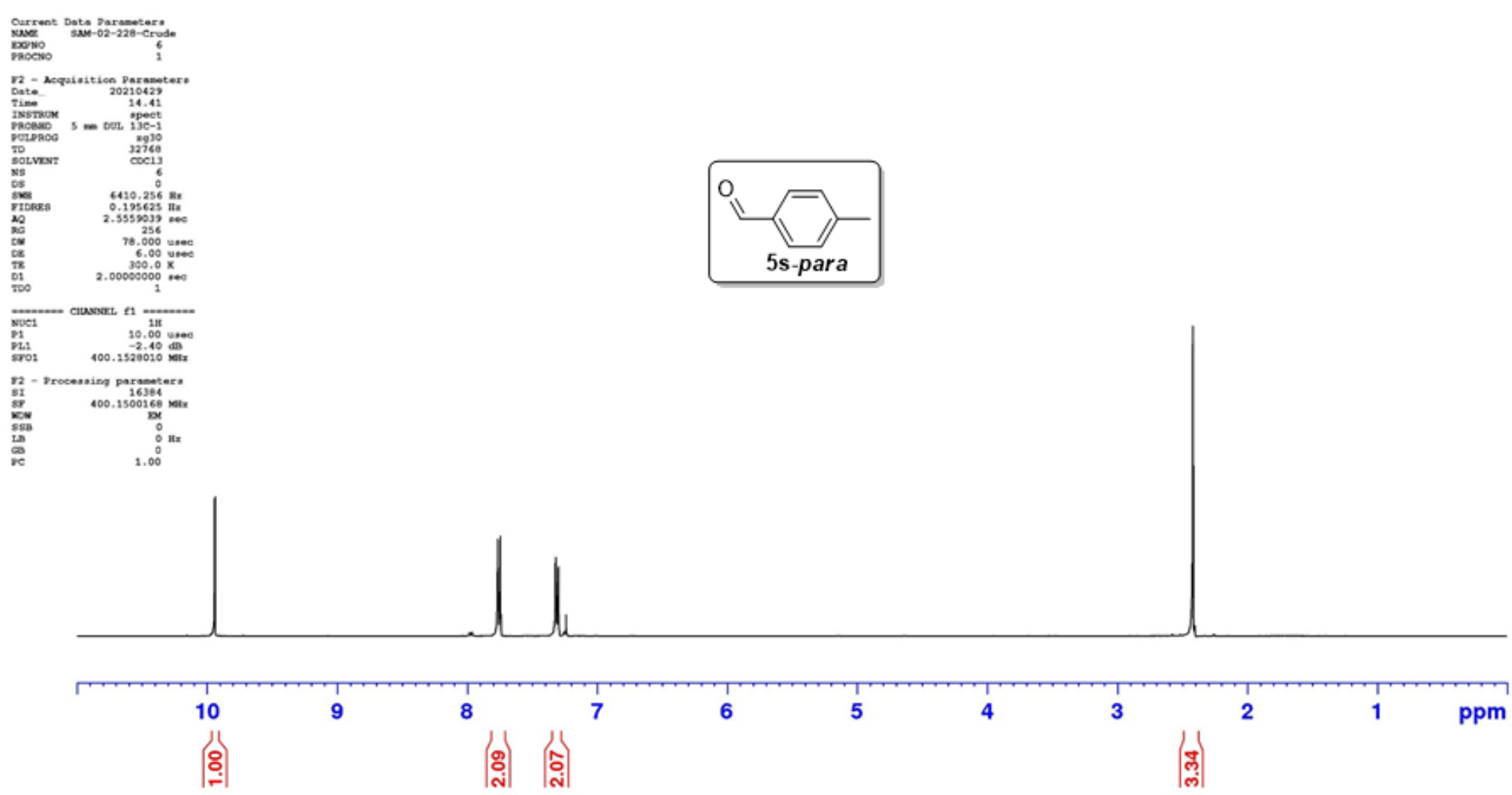


Solvent: $\mathrm{CDCl}_{3}$

SFO1: $175 \mathrm{MHz}$

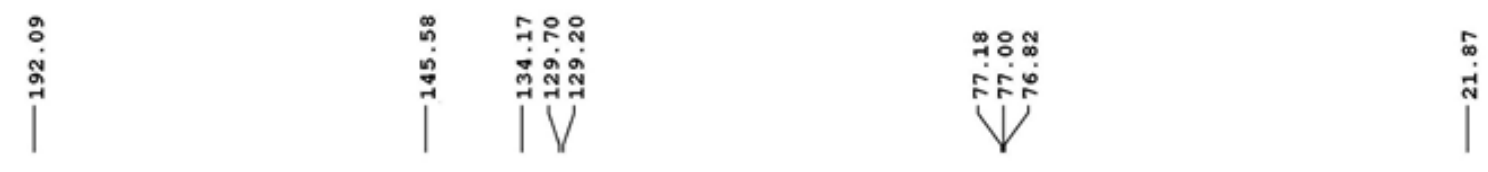

SAM-2-228-3
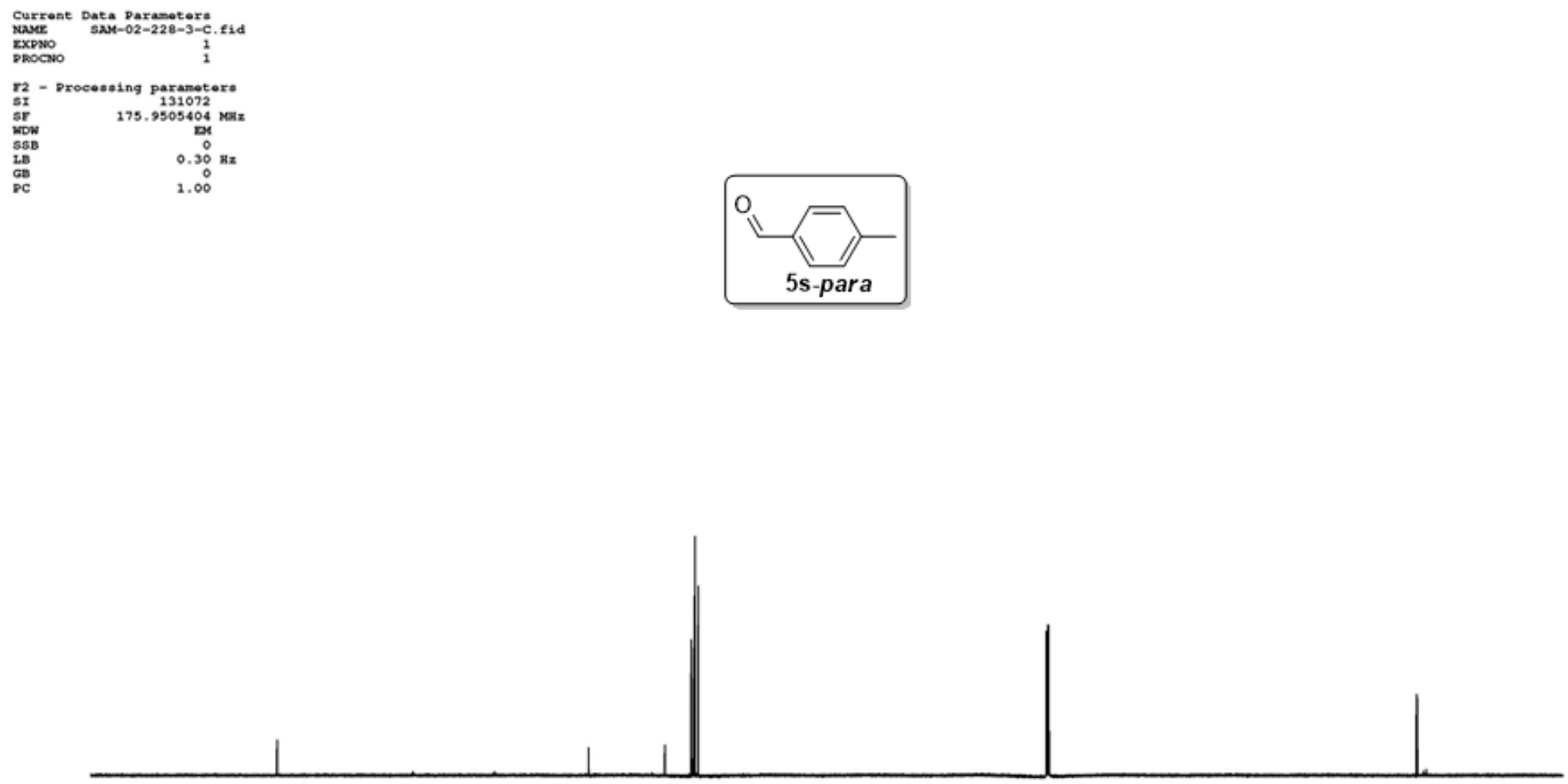

$$
\begin{array}{lllllllllllllllllllll}
210 & 200 & 190 & 180 & 170 & 160 & 150 & 140 & 130 & 120 & 110 & 100 & 90 & 80 & 70 & 60 & 50 & 40 & 30 & 20 & \mathrm{ppm}
\end{array}
$$


Solvent: $\mathrm{CDCl}_{3}$ SFO1: $400 \mathrm{MHz}$

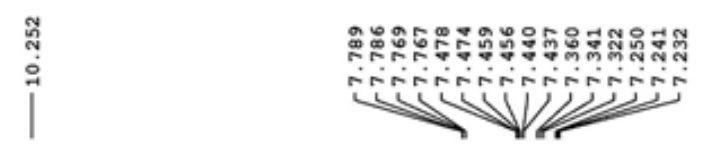

SAM-02-228-1

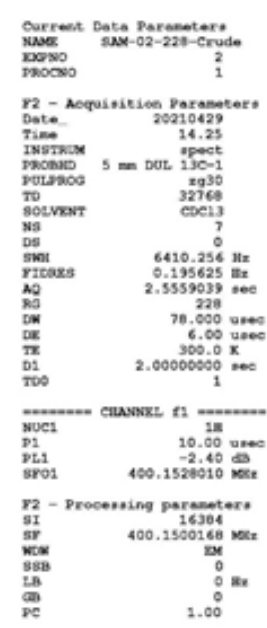

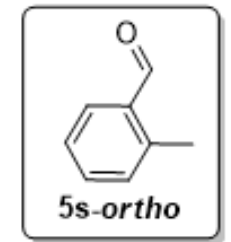

Lill

웅항

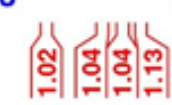
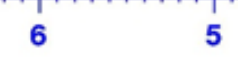

$\left|\frac{1}{\circ}\right|$ 
Solvent: $\mathrm{CDCl}_{3}$

SFO1: $100 \mathrm{MHz}$

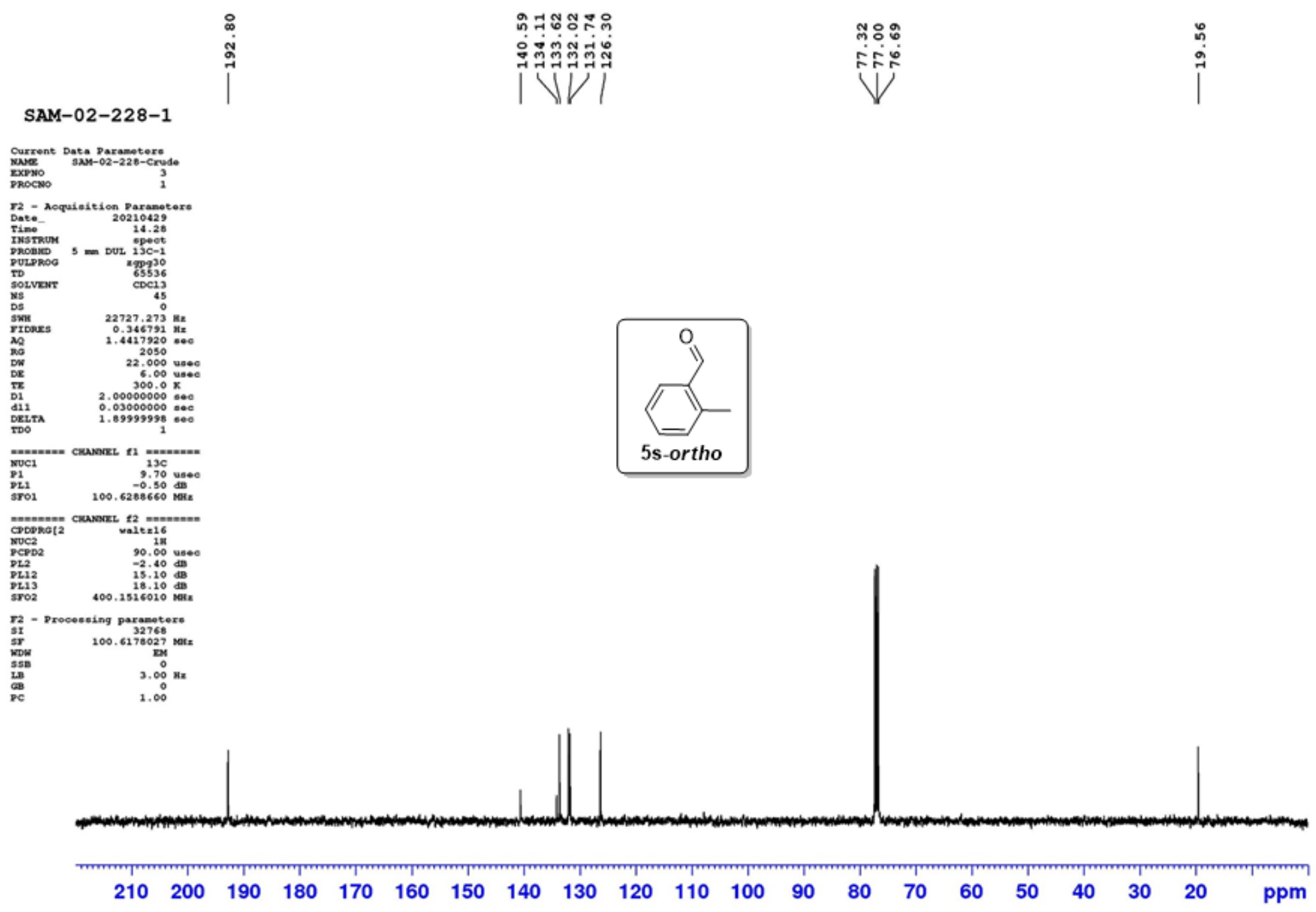


Solvent: $\mathrm{CDCl}_{3}$

SFO1: $400 \mathrm{MHz}$

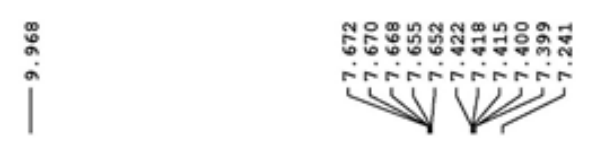

SAM-02-228-2
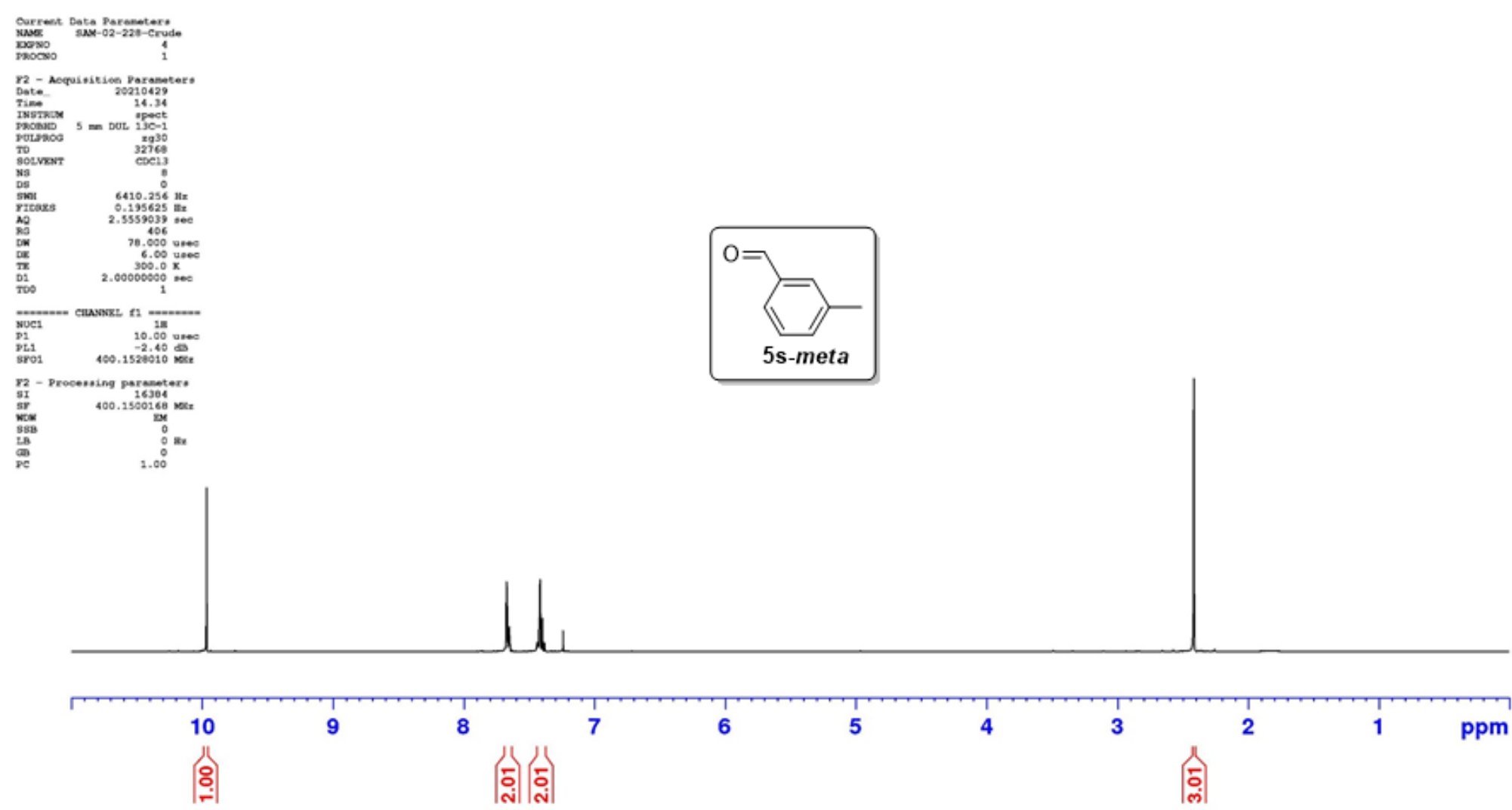
Solvent: $\mathrm{CDCl}_{3}$

SFO1: $100 \mathrm{MHz}$

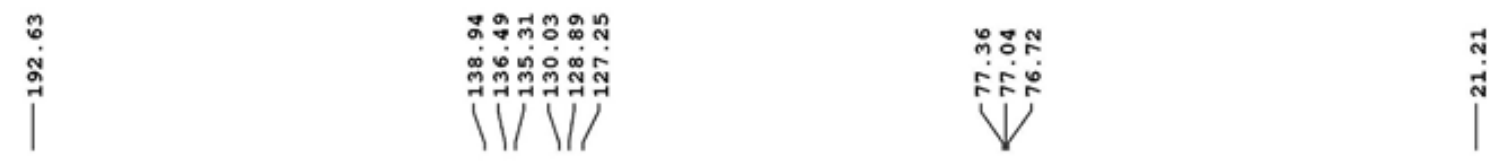

SAM-02-228-2
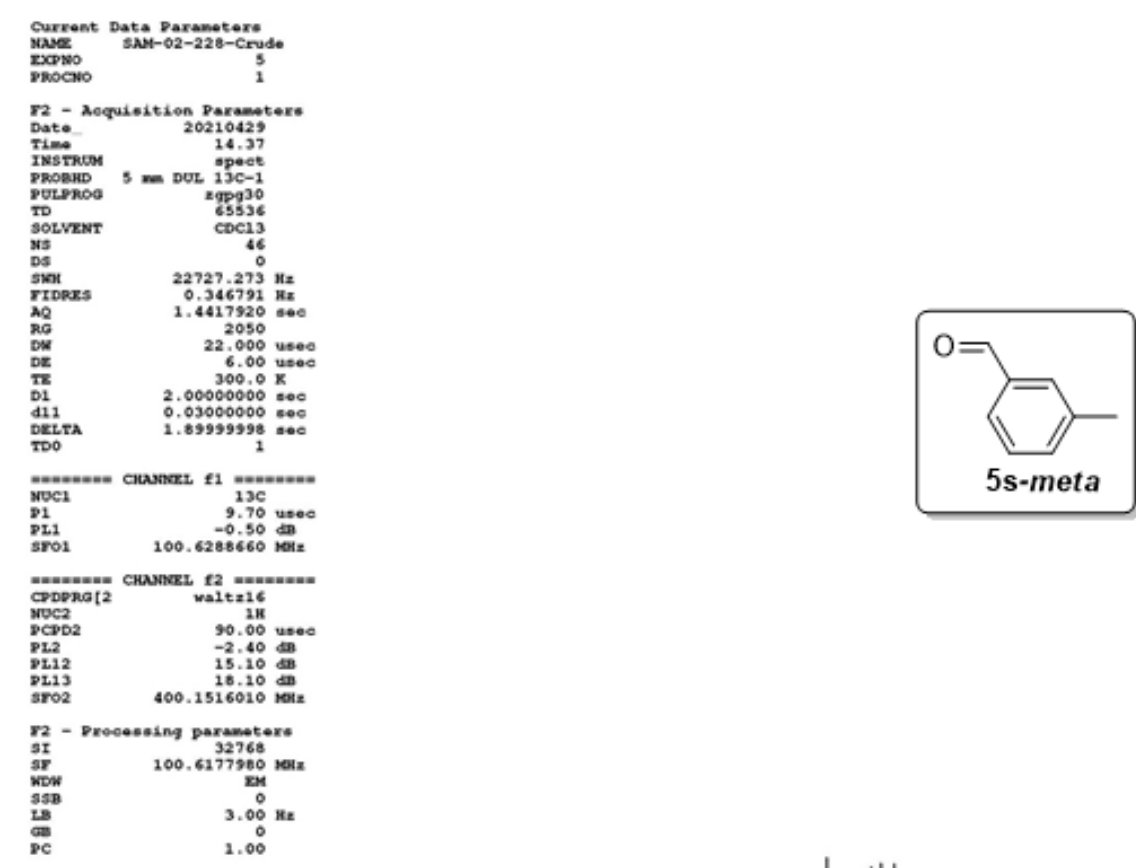
Solvent: $\mathrm{CDCl}_{3}$

SFO1: $700 \mathrm{MHz}$

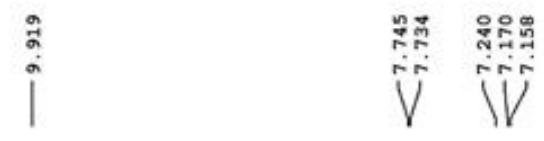

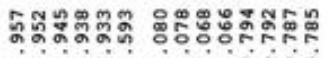

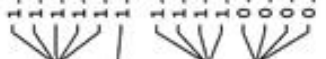
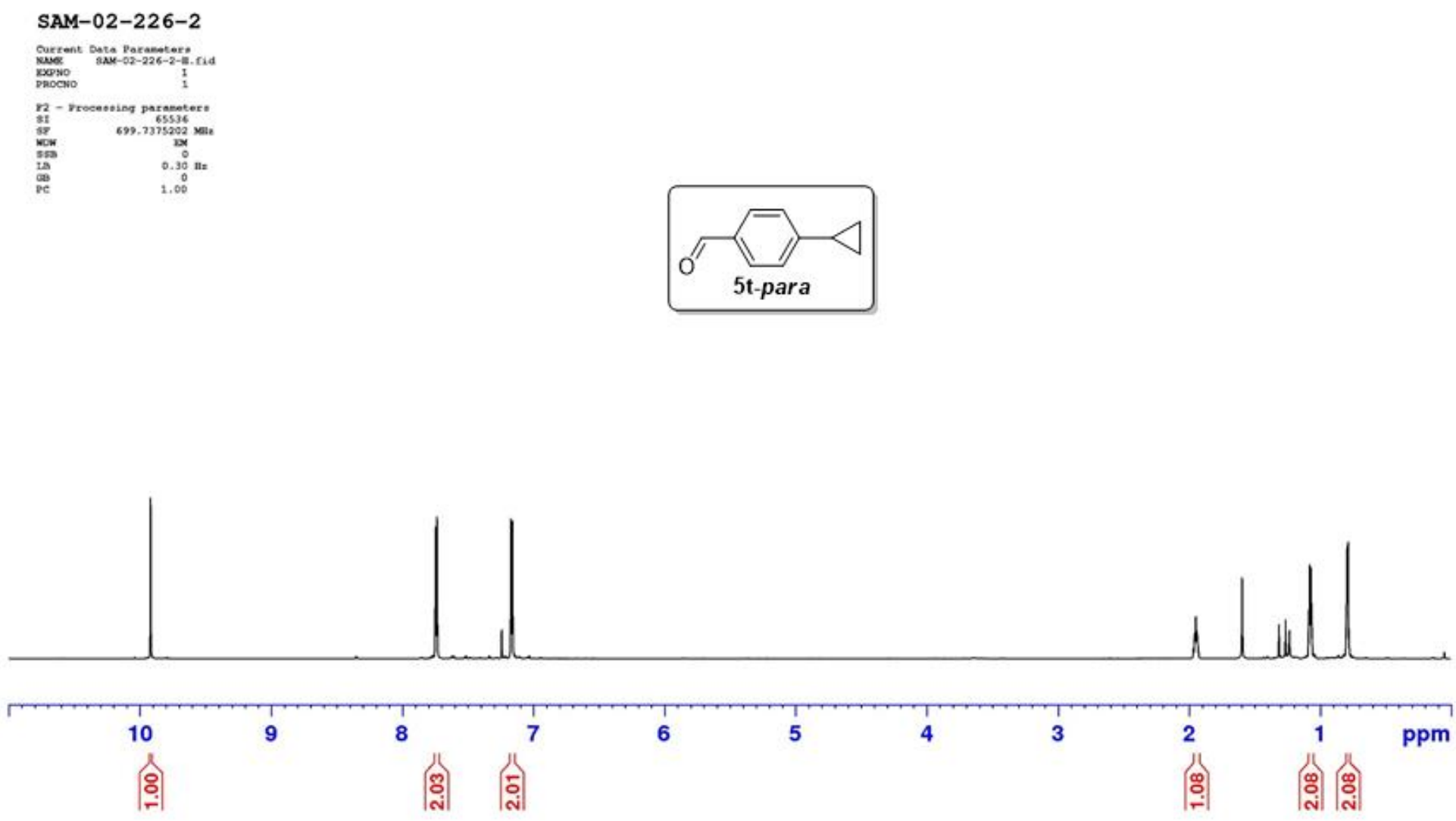
Solvent: $\mathrm{CDCl}_{3}$

SFO1: $175 \mathrm{MHz}$

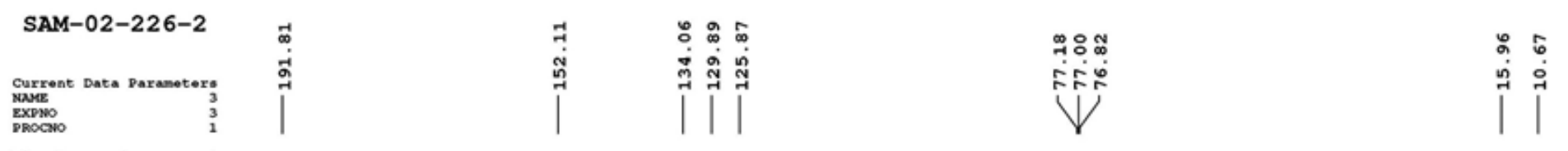

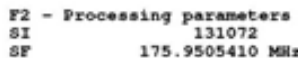

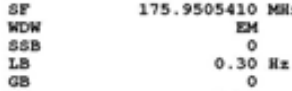

1.00

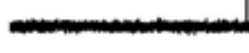

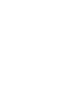
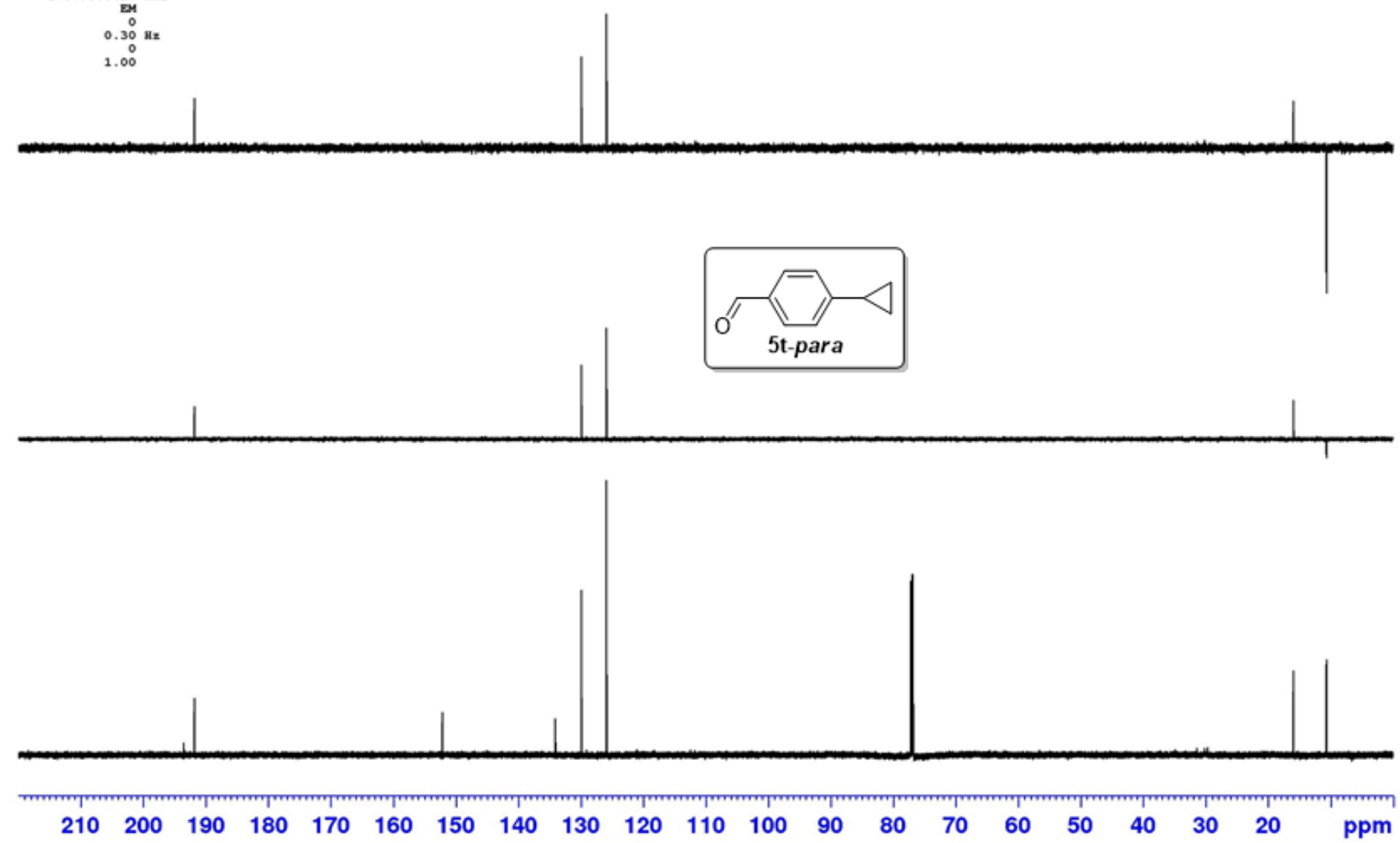
Solvent: $\mathrm{CDCl}_{3}$

SFO1: $400 \mathrm{MHz}$

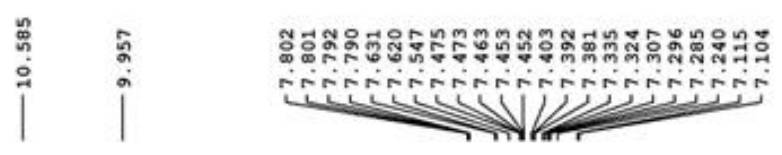

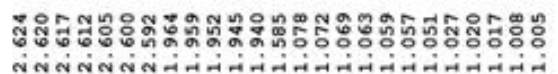

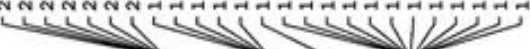

\section{SAM-02-226-1}

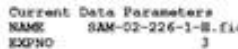

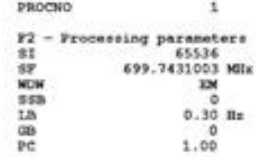
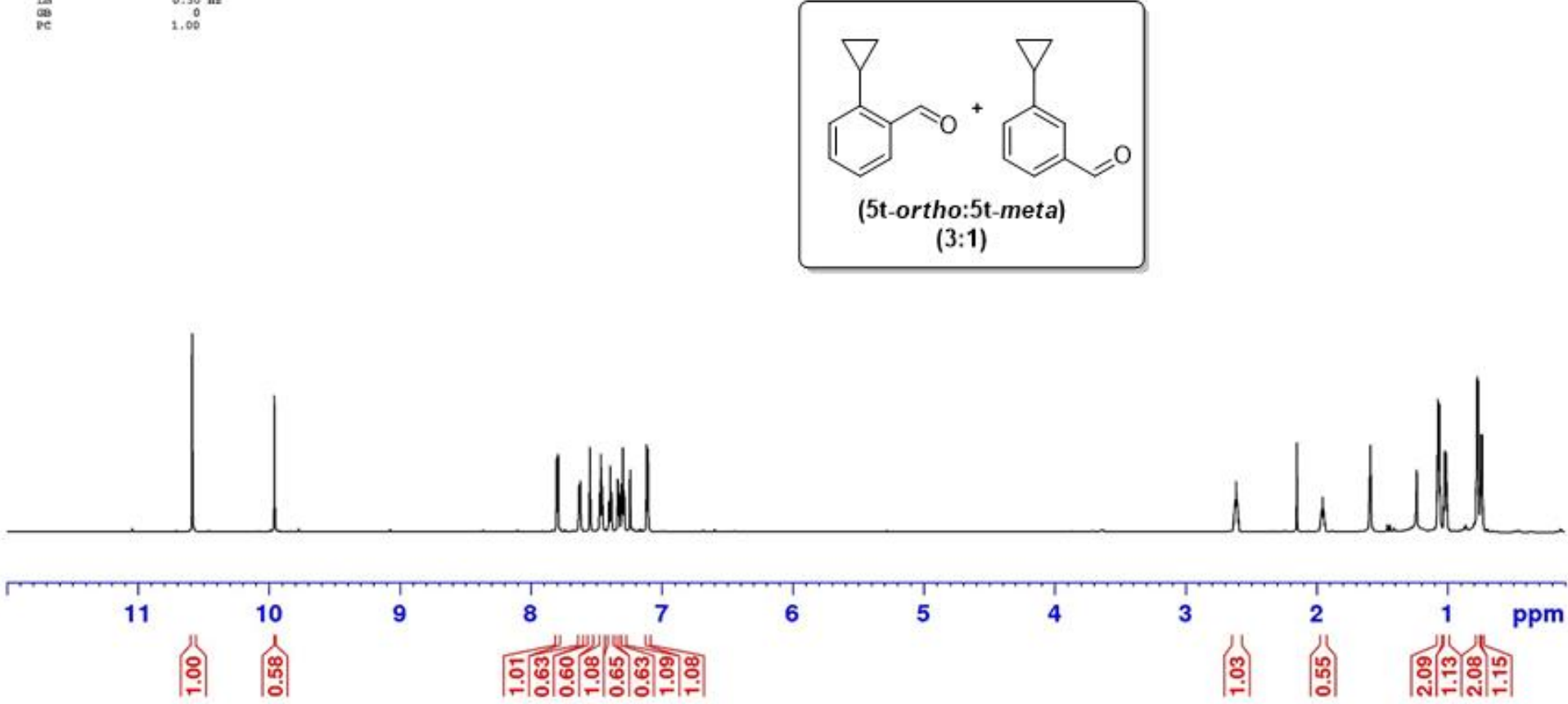
Solvent: $\mathrm{CDCl}_{3}$

SFO1: $175 \mathrm{MHz}$

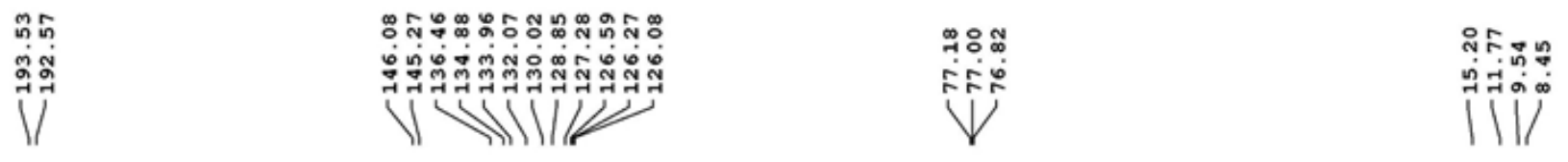

SAM-02-226-1
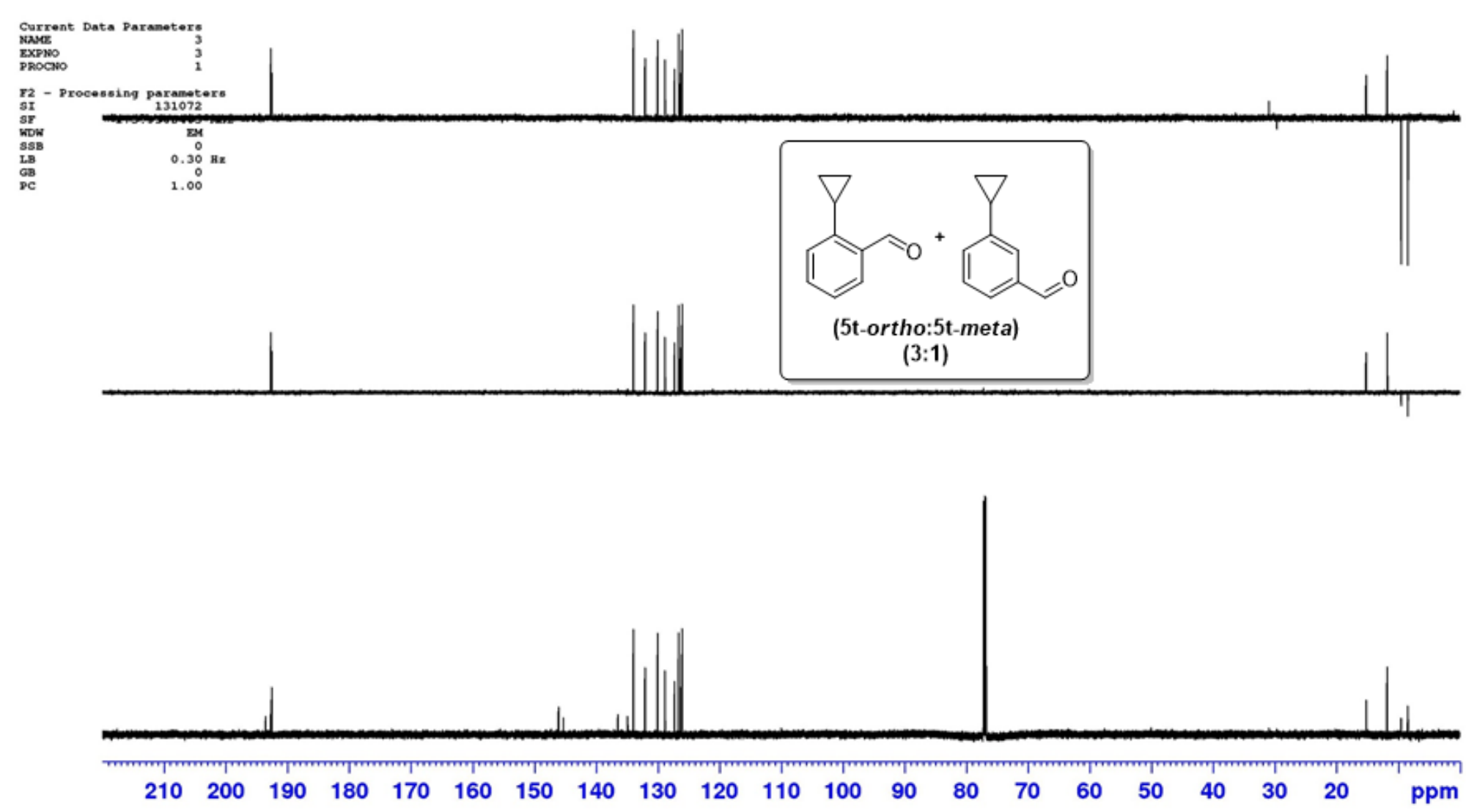
Solvent: $\mathrm{CDCl}_{3}$ SFO1: $600 \mathrm{MHz}$

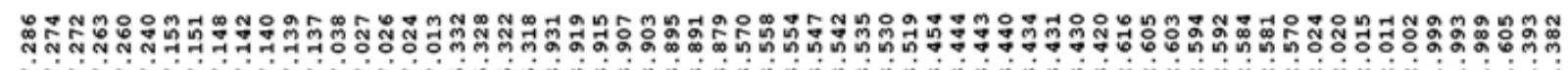

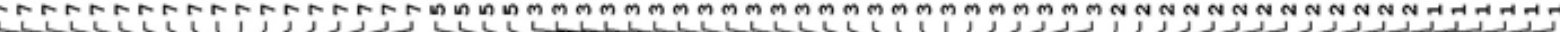

SAM-02-106-H-minor
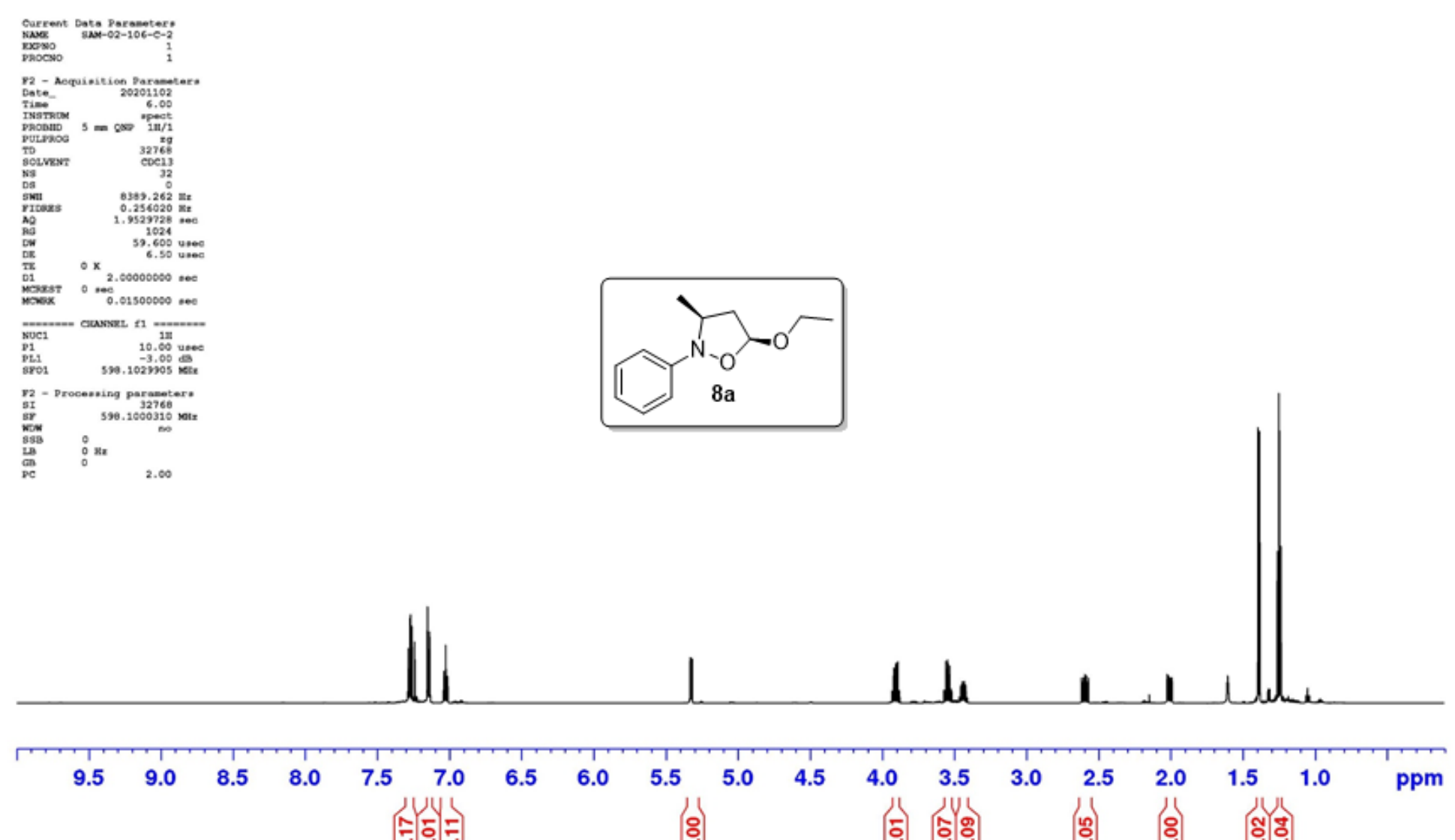

$9.5 \quad 9.0$

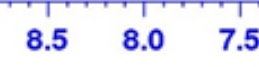

천ํำ:

6.5

항

5.

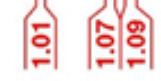

욤

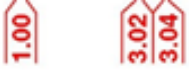


Solvent: $\mathrm{CDCl}_{3}$ SFO1: $175 \mathrm{MHz}$

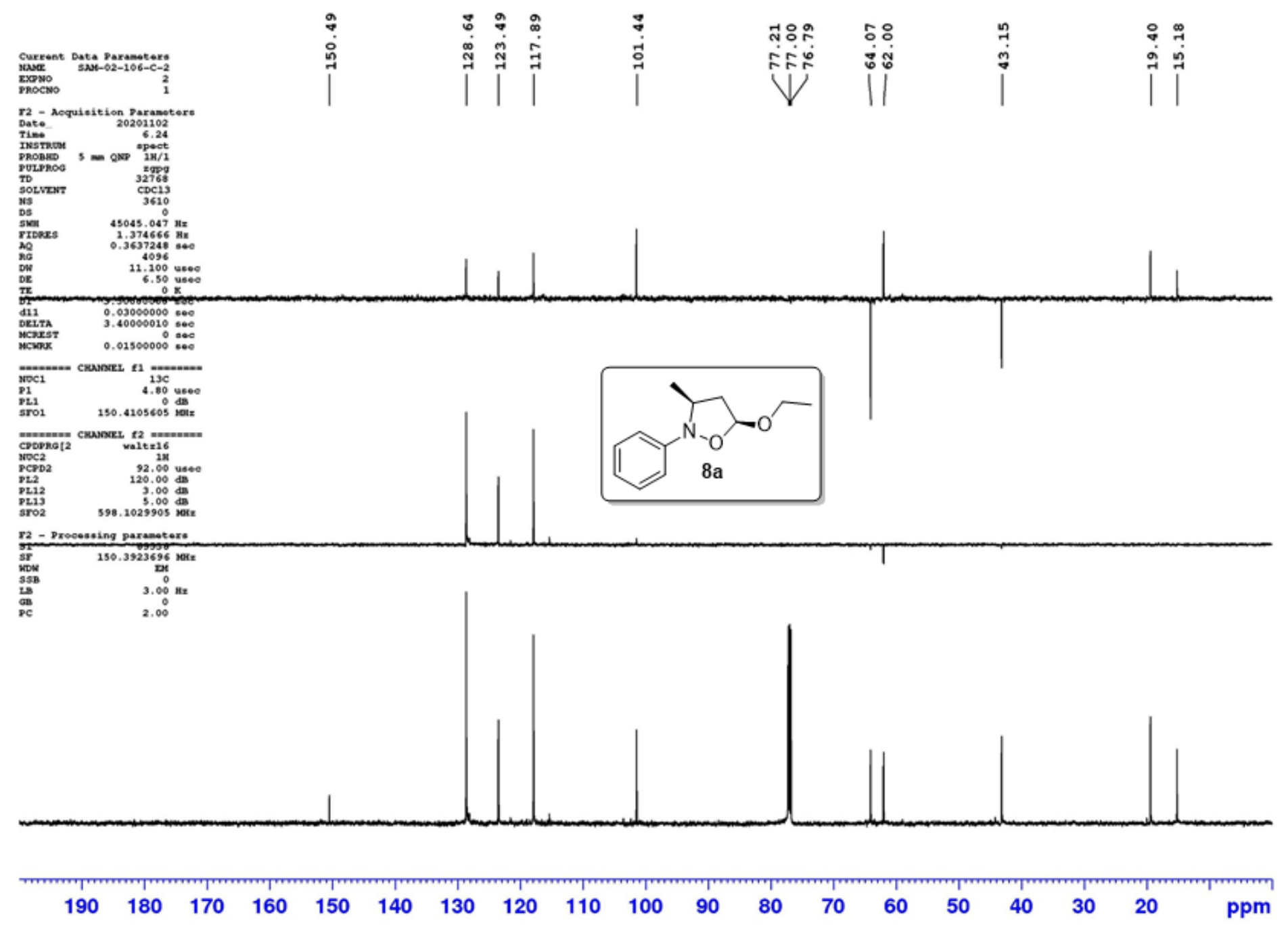


Solvent: $\mathrm{CDCl}_{3}$ SFO1: $700 \mathrm{MHz}$

;AM-02-106-:

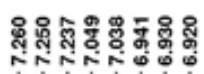

VVV

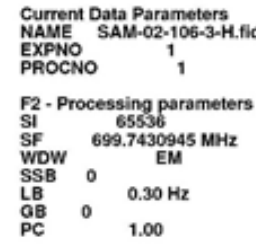

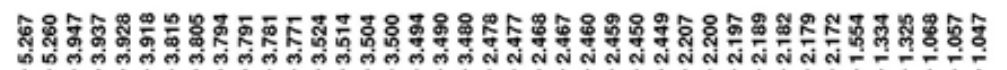

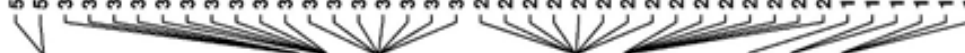

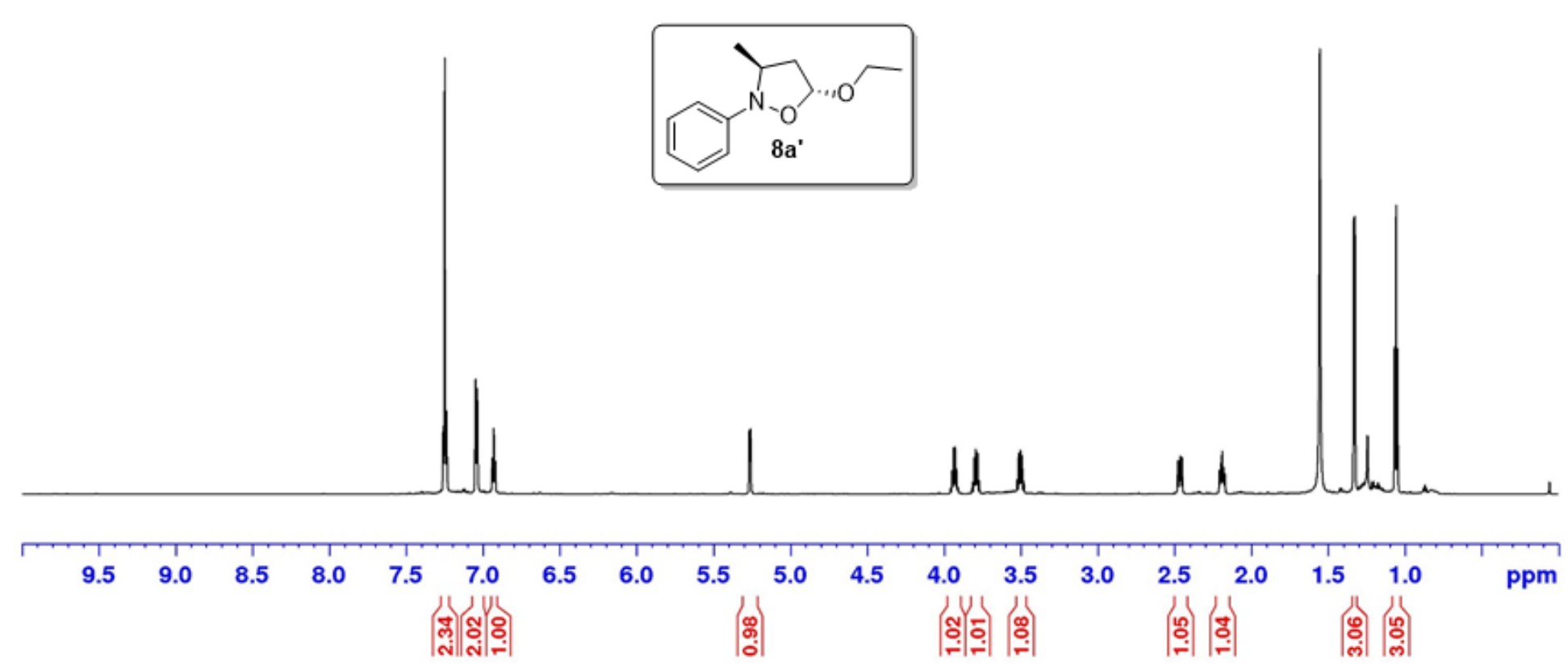


Solvent: $\mathrm{CDCl}_{3}$

SFO1: $175 \mathrm{MHz}$

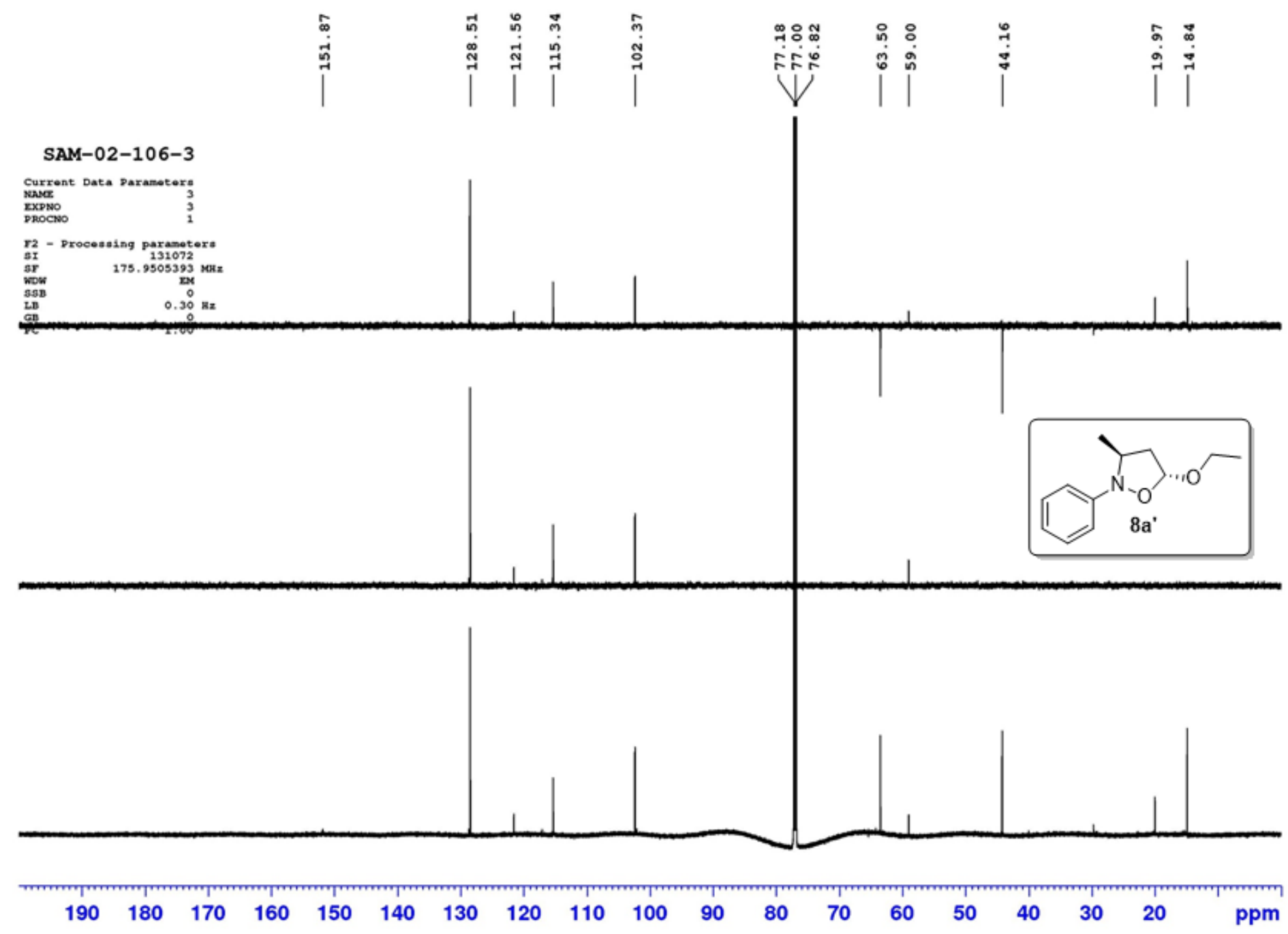


Solvent: $\mathrm{CDCl}_{3}$ SFO1: $700 \mathrm{MHz}$

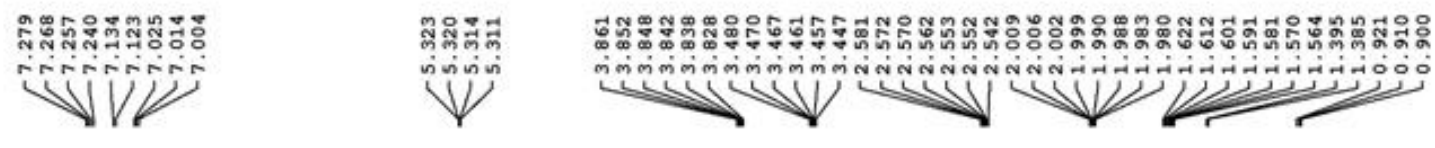

SAM-02-111-1

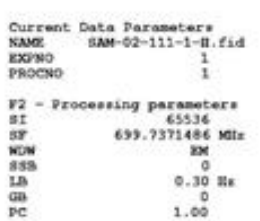
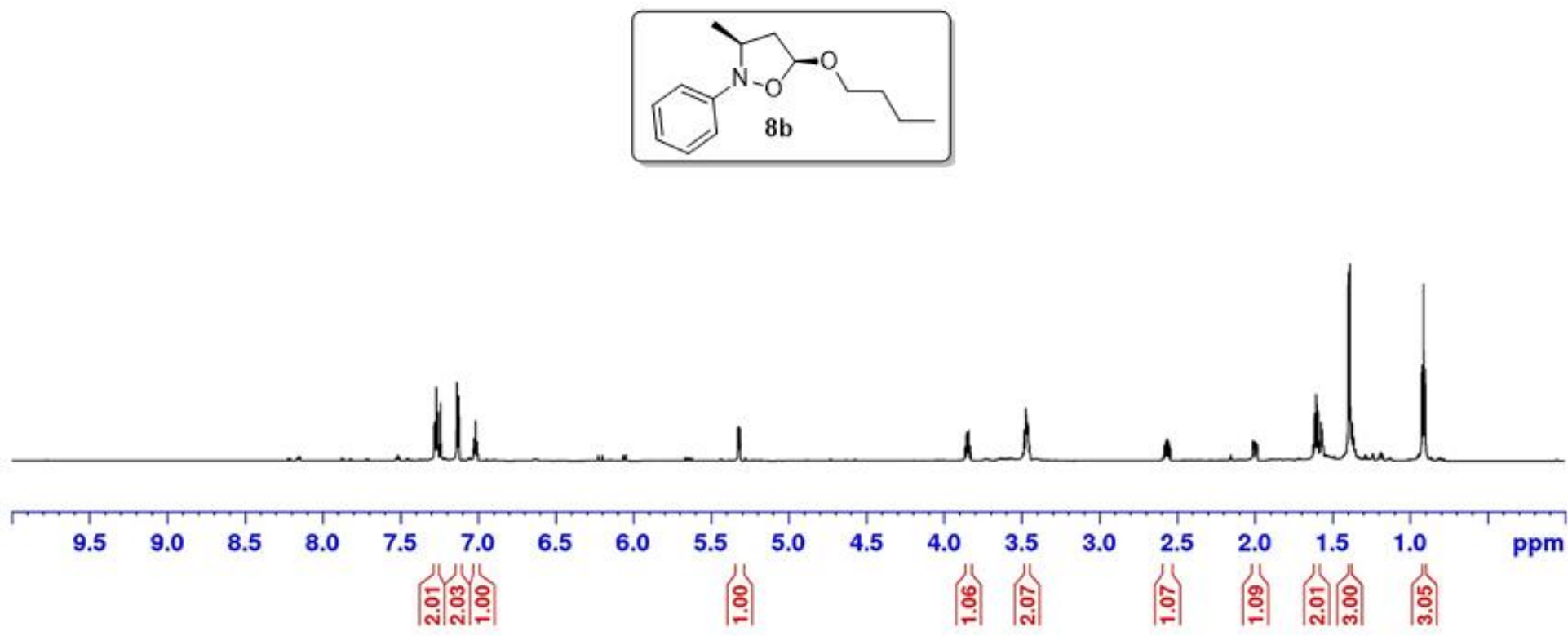
Solvent: $\mathrm{CDCl}_{3}$

SFO1: $175 \mathrm{MHz}$

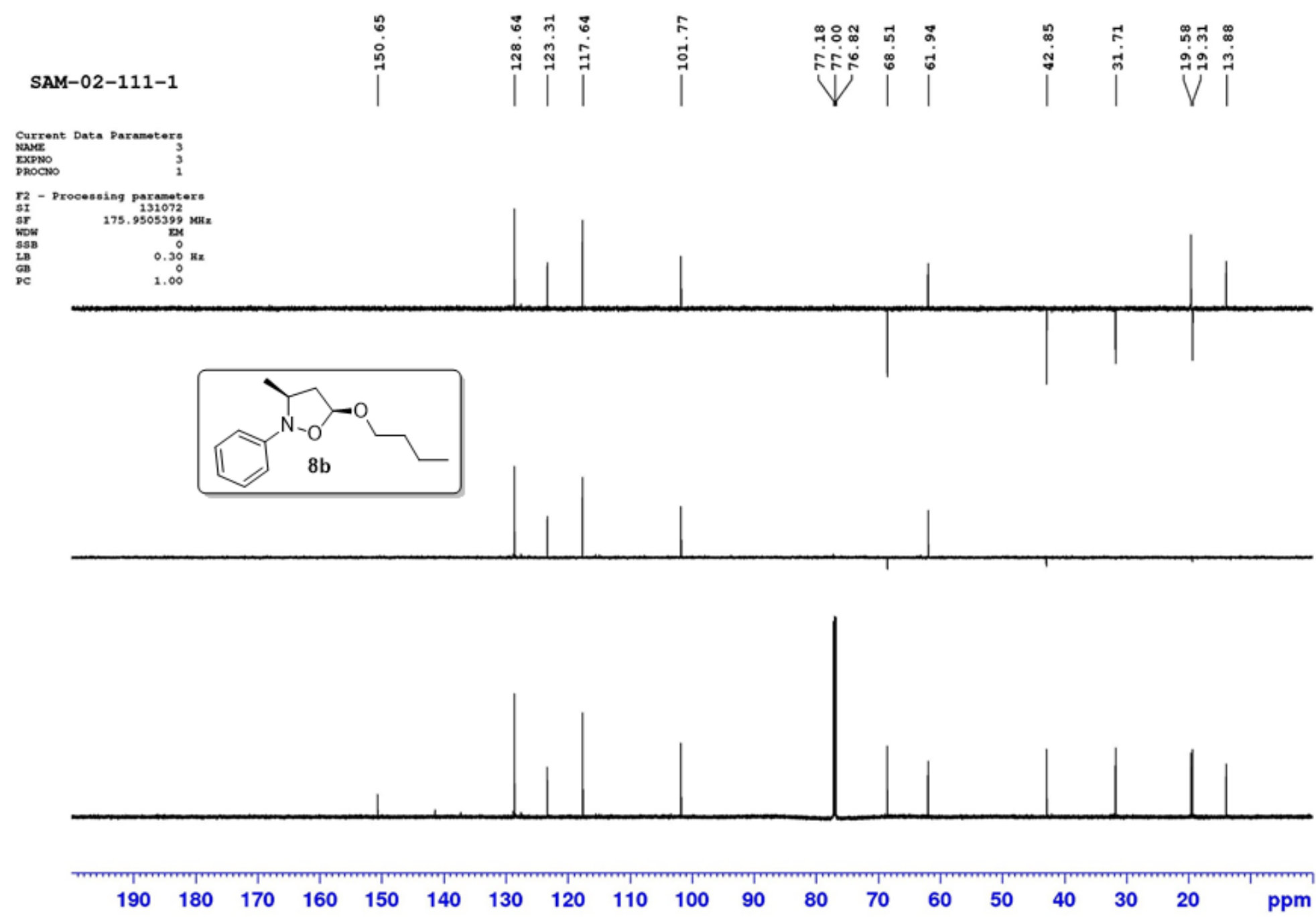


Solvent: $\mathrm{CDCl}_{3}$ SFO1: $700 \mathrm{MHz}$
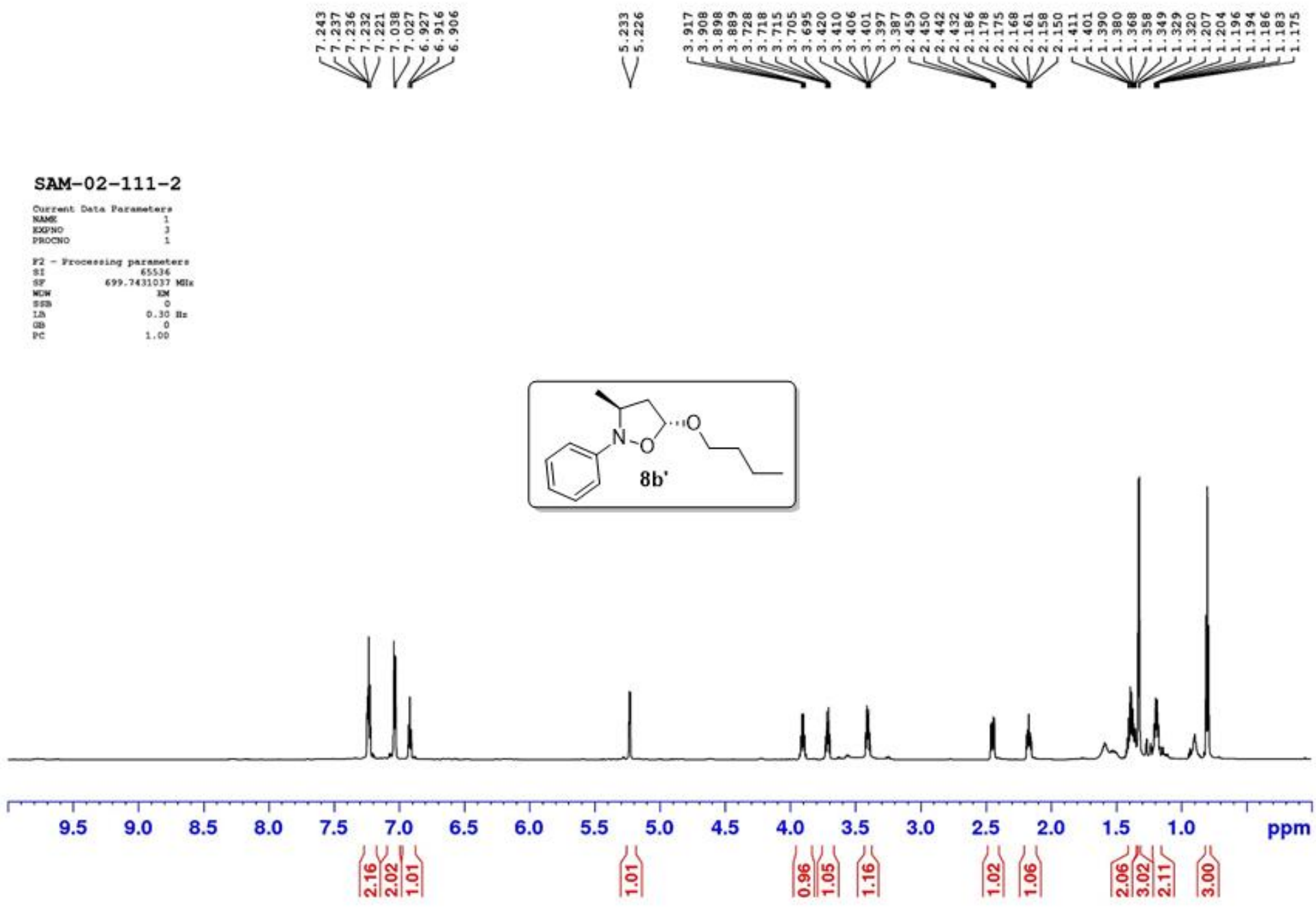
Solvent: $\mathrm{CDCl}_{3}$

SFO1: $175 \mathrm{MHz}$

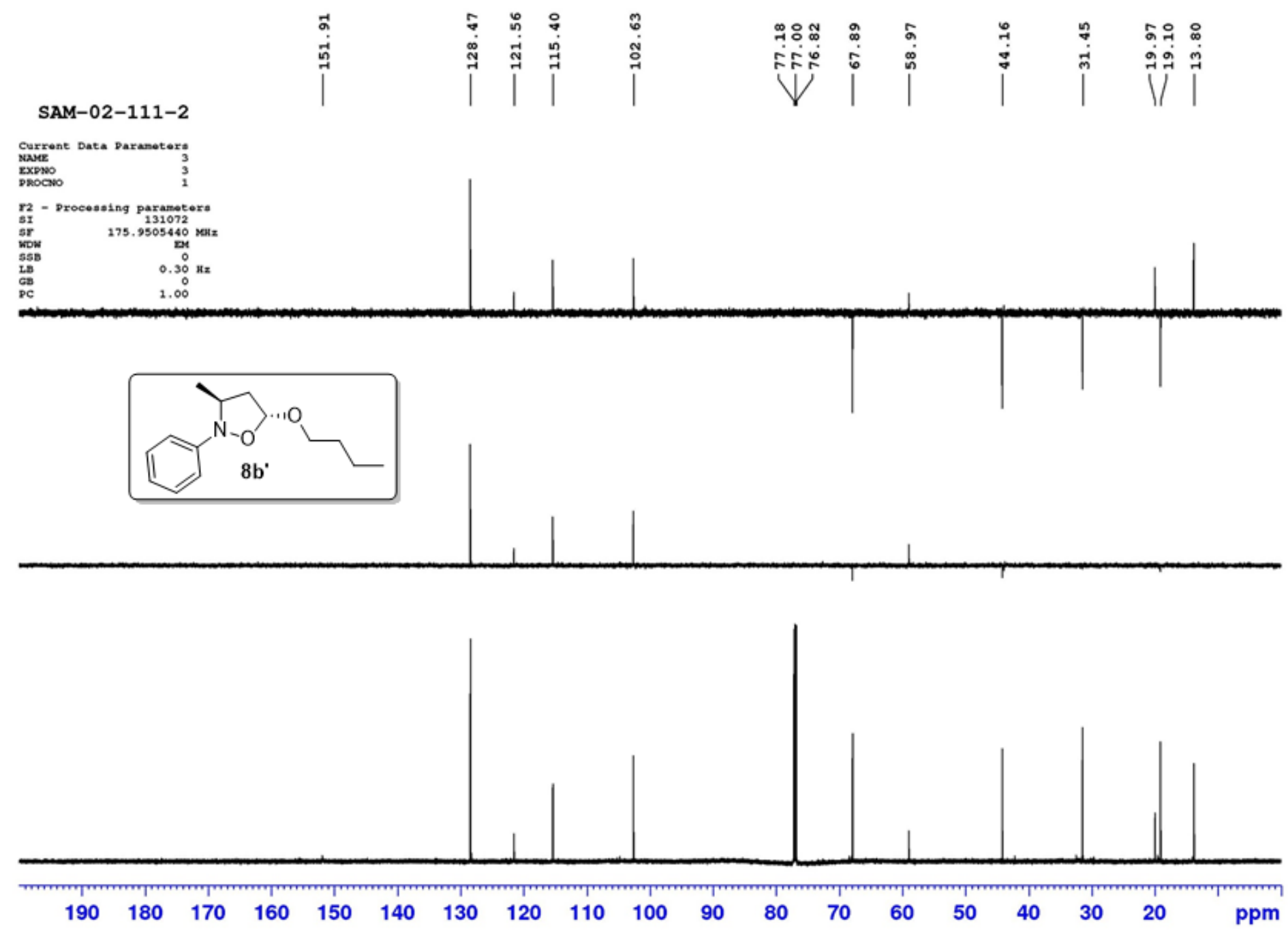


Solvent: $\mathrm{CDCl}_{3}$ SFO1: $700 \mathrm{MHz}$

줐줐

Nivin

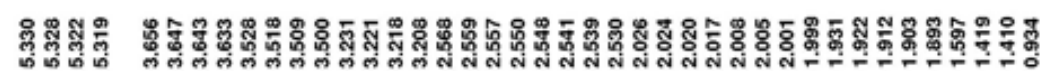

SAM-02-135-1

Current Data Parameters
NAME SAM-02-135-1-H.fid

PXPNO 1

F2 - Processing parameters

WF $699.7430945 \mathrm{MH}$

LB $0.30 \mathrm{~Hz}$

$\mathrm{PC} \quad 1.00$

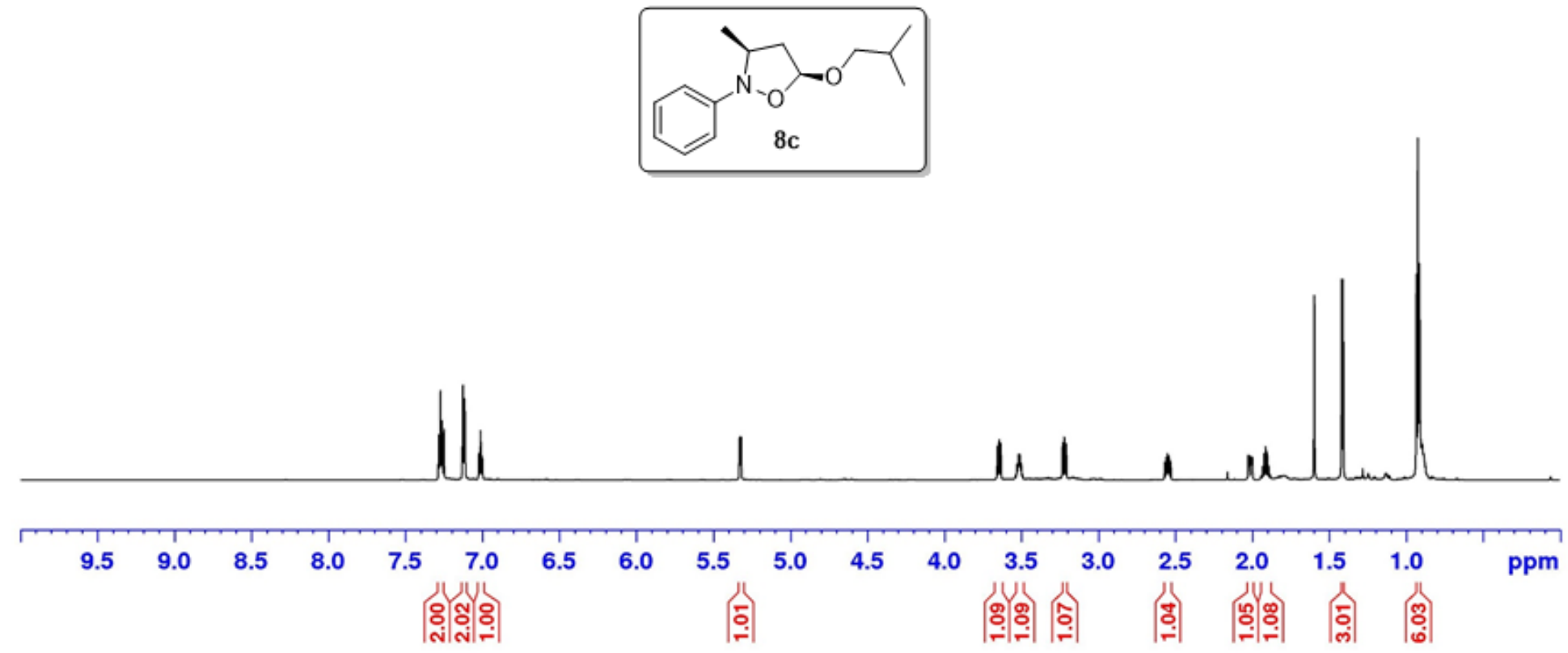


Solvent: $\mathrm{CDCl}_{3}$

SFO1: $175 \mathrm{MHz}$
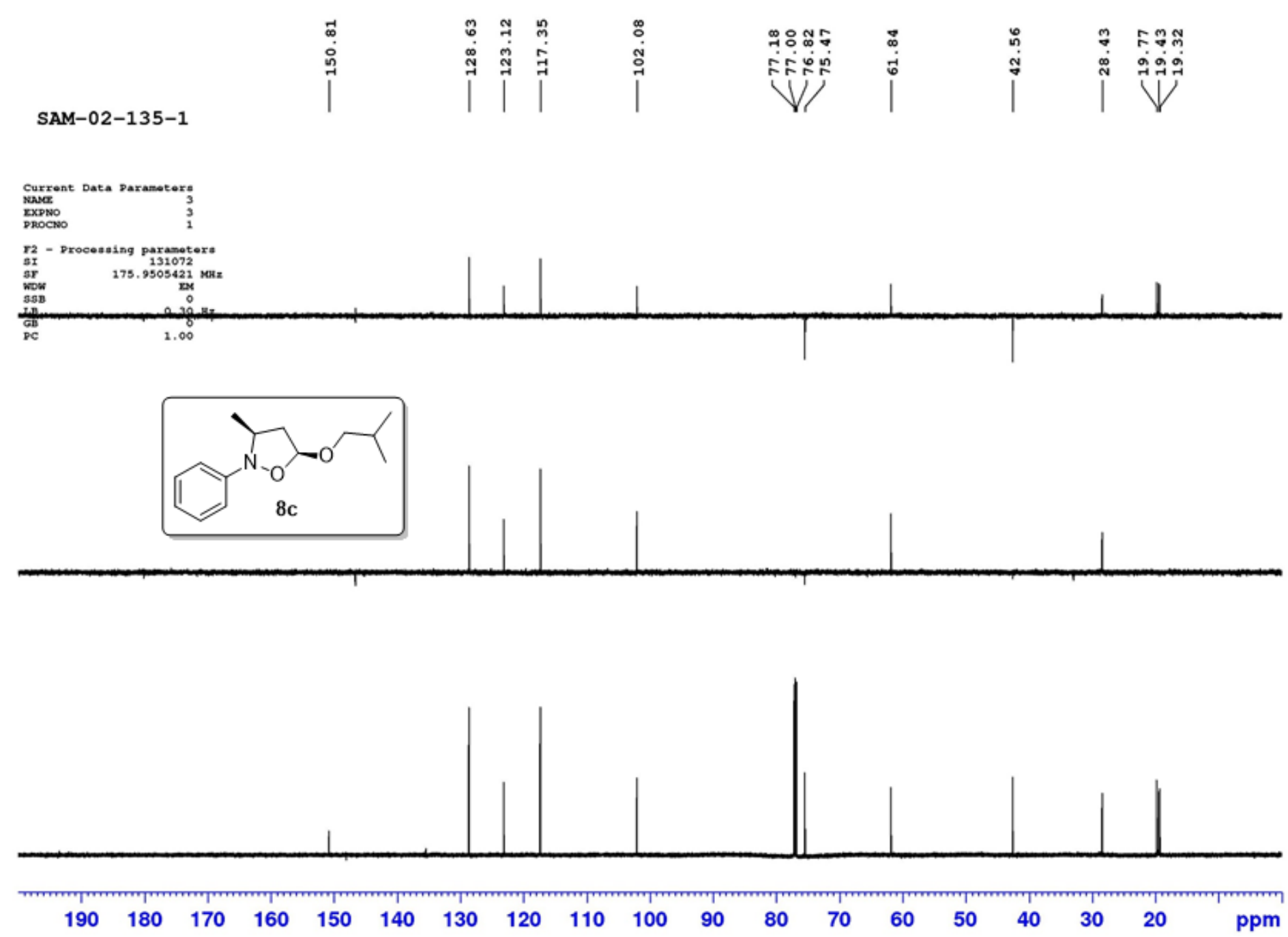
Solvent: $\mathrm{CDCl}_{3}$

SFO1: $700 \mathrm{MHz}$

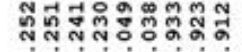

FiNTFE0

V

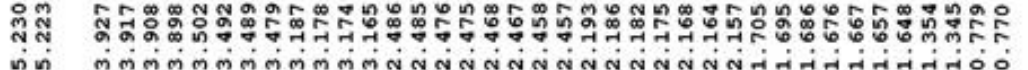



\section{SAM-02-135-2}

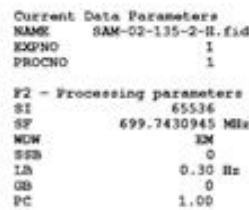
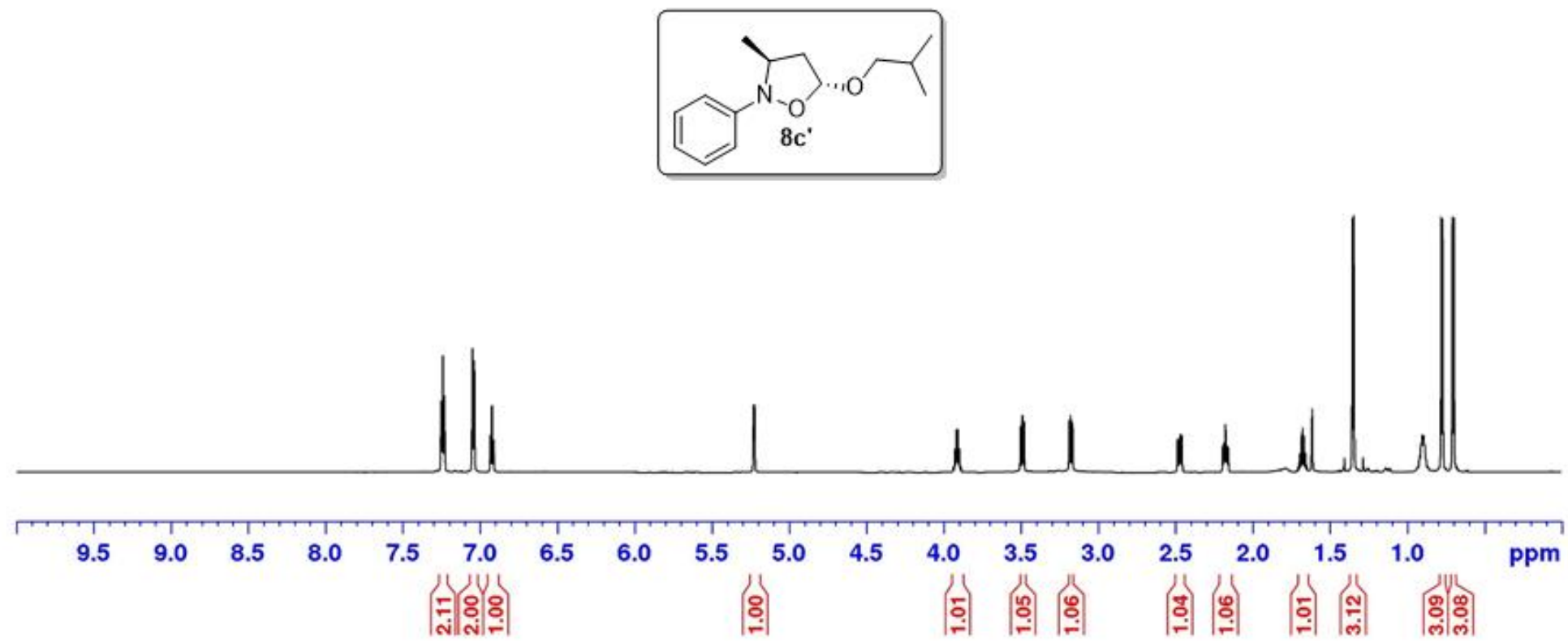
Solvent: $\mathrm{CDCl}_{3}$

SFO1: $175 \mathrm{MHz}$
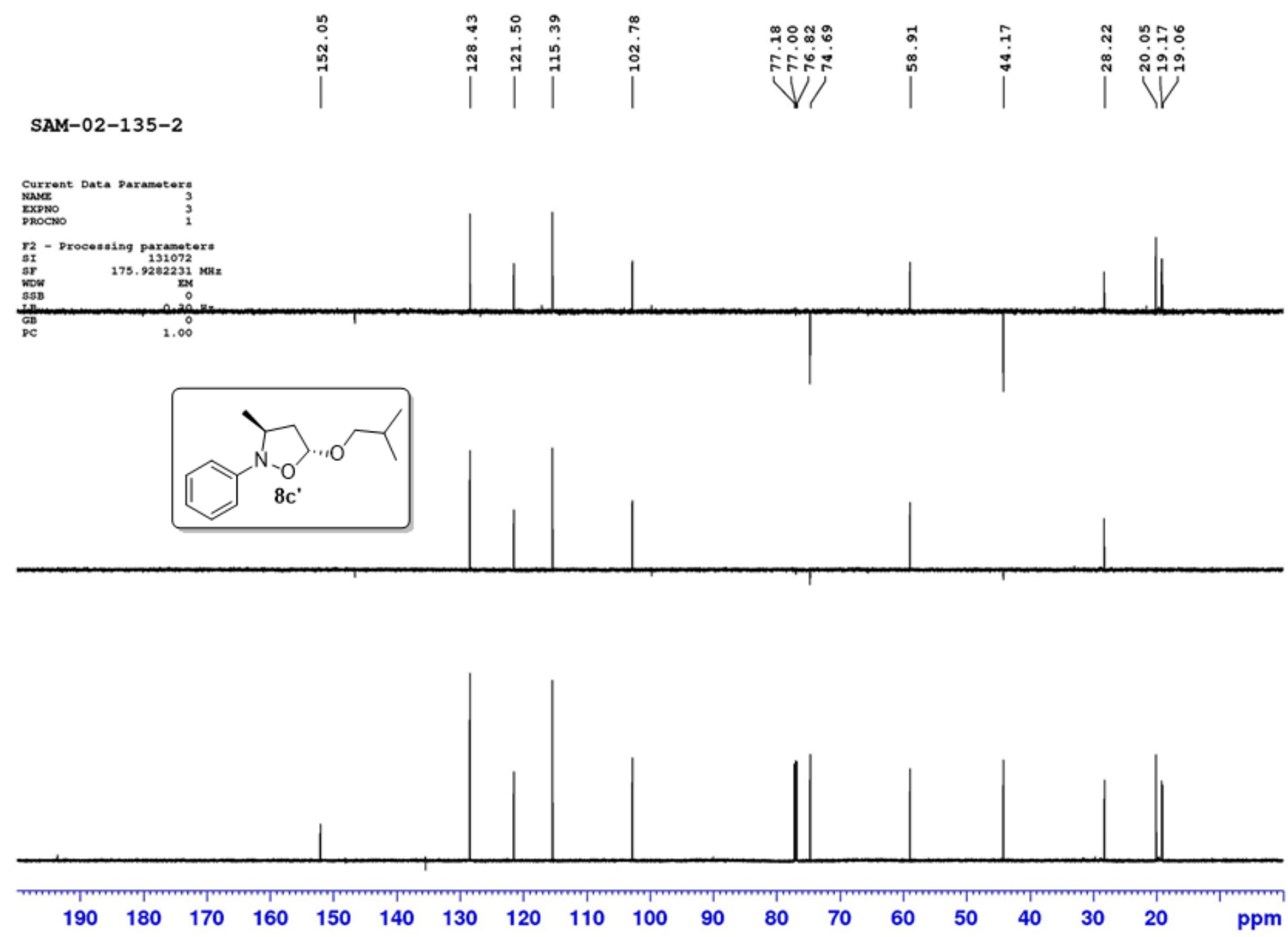
Solvent: $\mathrm{CDCl}_{3}$ SFO1: $700 \mathrm{MHz}$

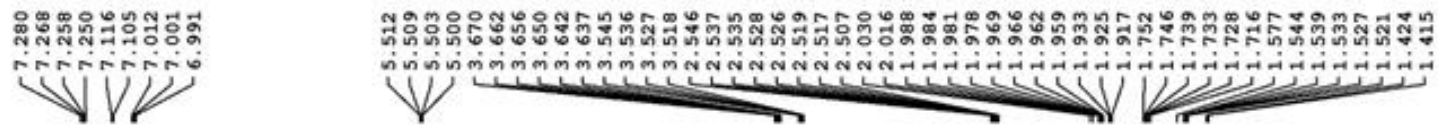

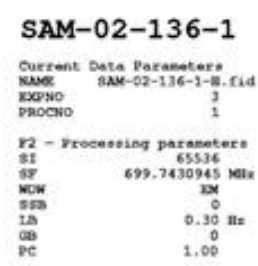
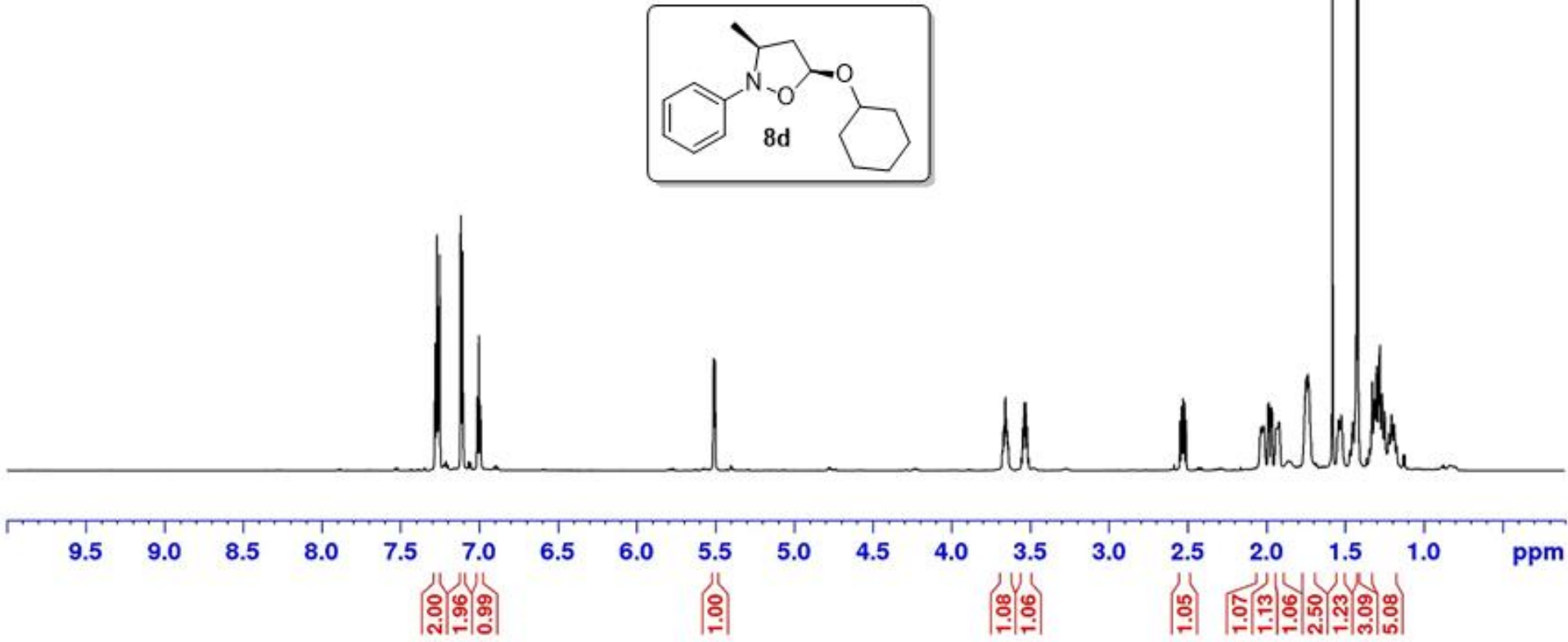
Solvent: $\mathrm{CDCl}_{3}$

SFO1: $175 \mathrm{MHz}$

|

SAM-02-136-1-C

Current Data Parameters

NAME

EXPNO

F2 - Processing parameters

SF $\quad 175.9505402 \mathrm{MHz}$

WDW EM

$\begin{array}{lll}\text { SSB } & 0 & 0.30 \mathrm{~Hz}\end{array}$

$\begin{array}{lll}\text { GB } & 0 & 0.30 \\ \text { PC } & 1.00\end{array}$
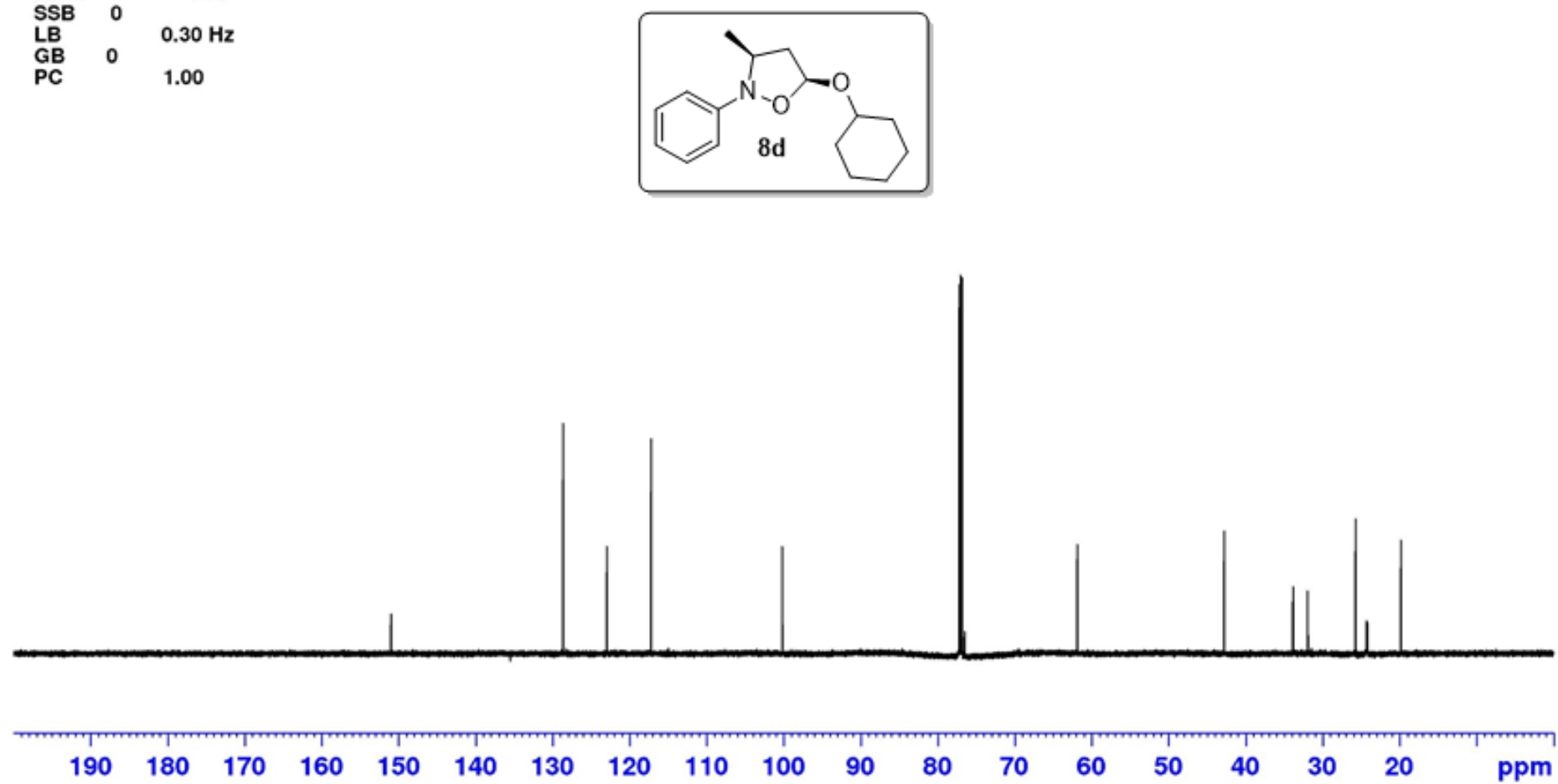
Solvent: $\mathrm{CDCl}_{3}$ SFO1: $700 \mathrm{MHz}$

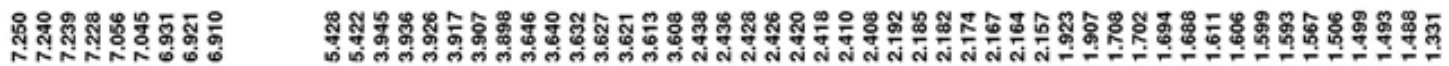

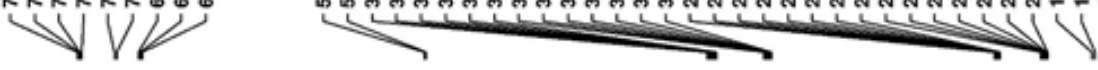

SAM-02-136-2

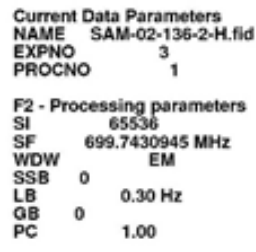

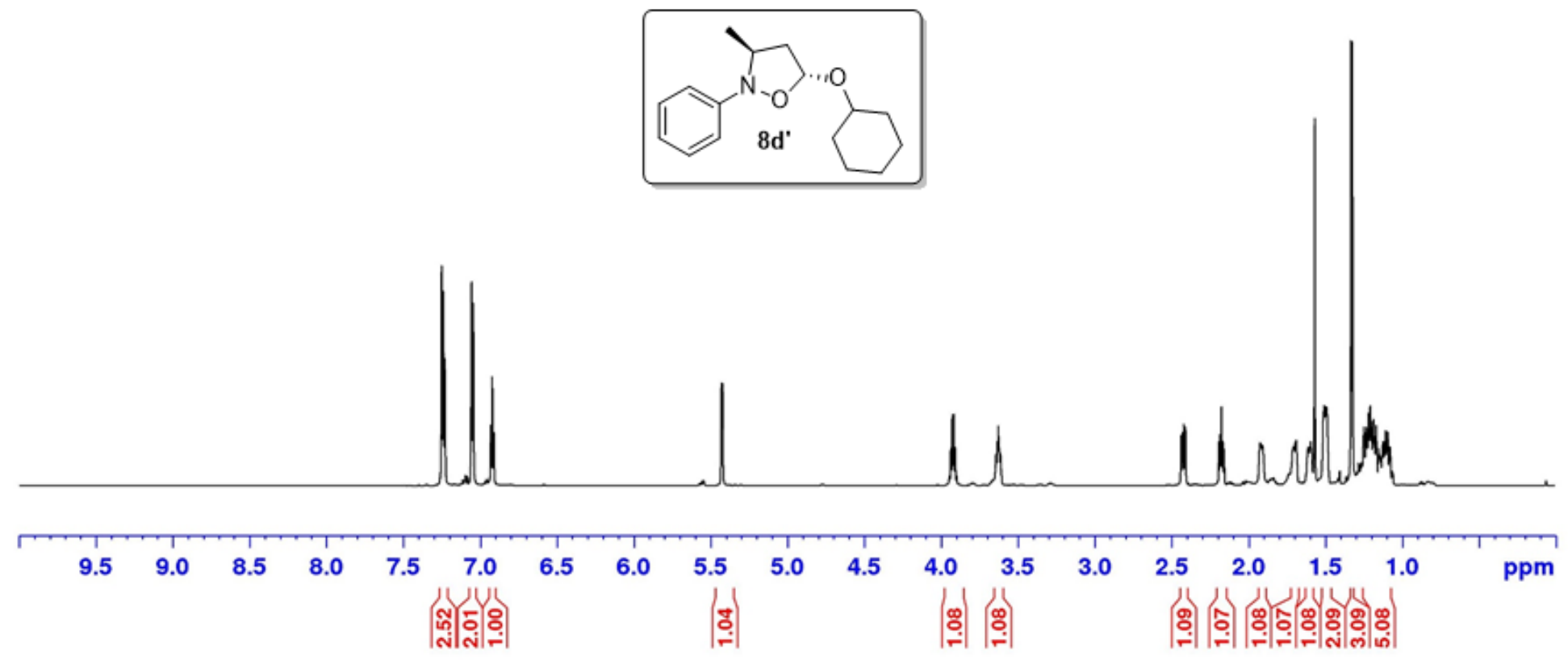


Solvent: $\mathrm{CDCl}_{3}$

SFO1: $175 \mathrm{MHz}$

SAM-02-136-2-C
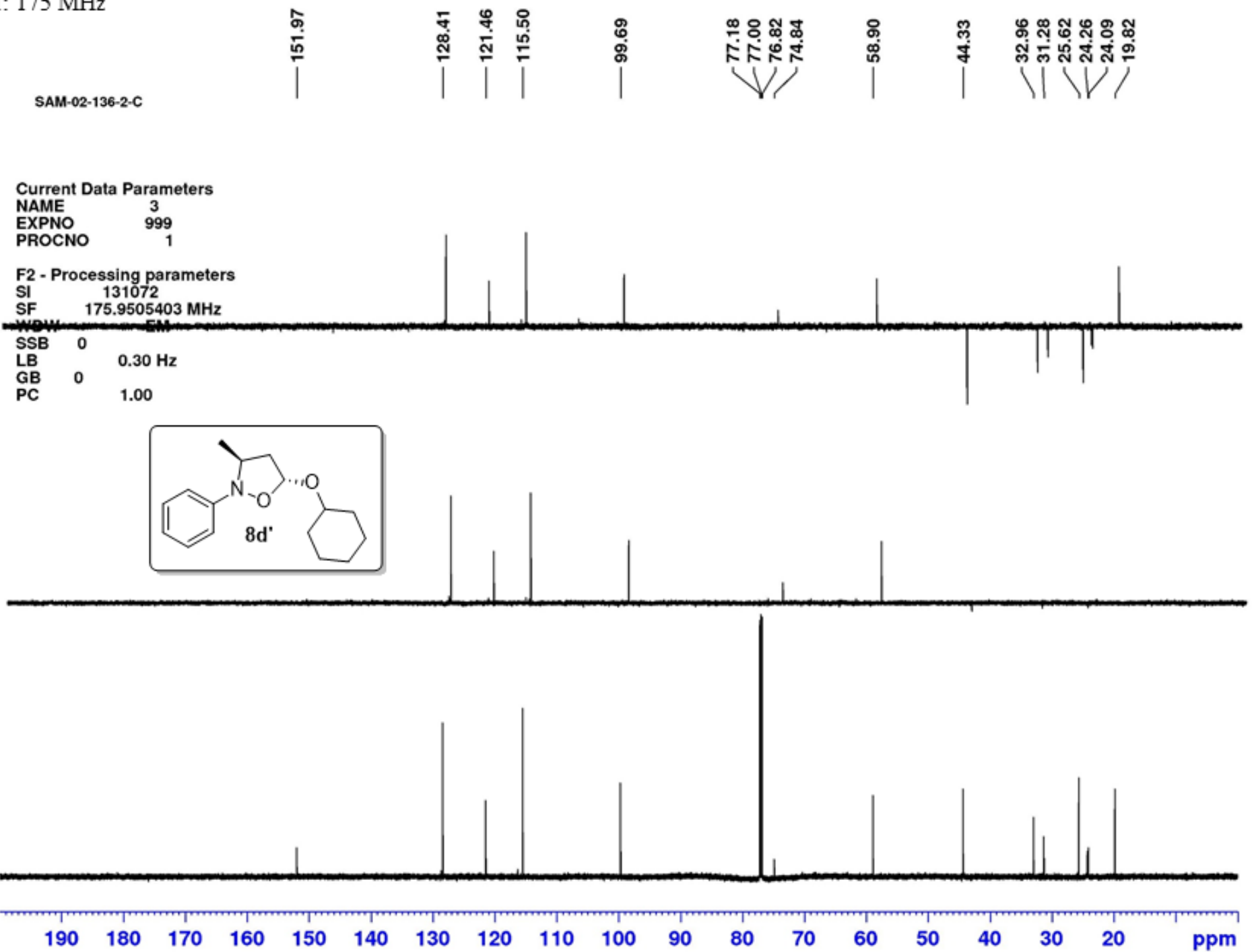
Solvent: $\mathrm{CDCl}_{3}$

SFO1: $400 \mathrm{MHz}$

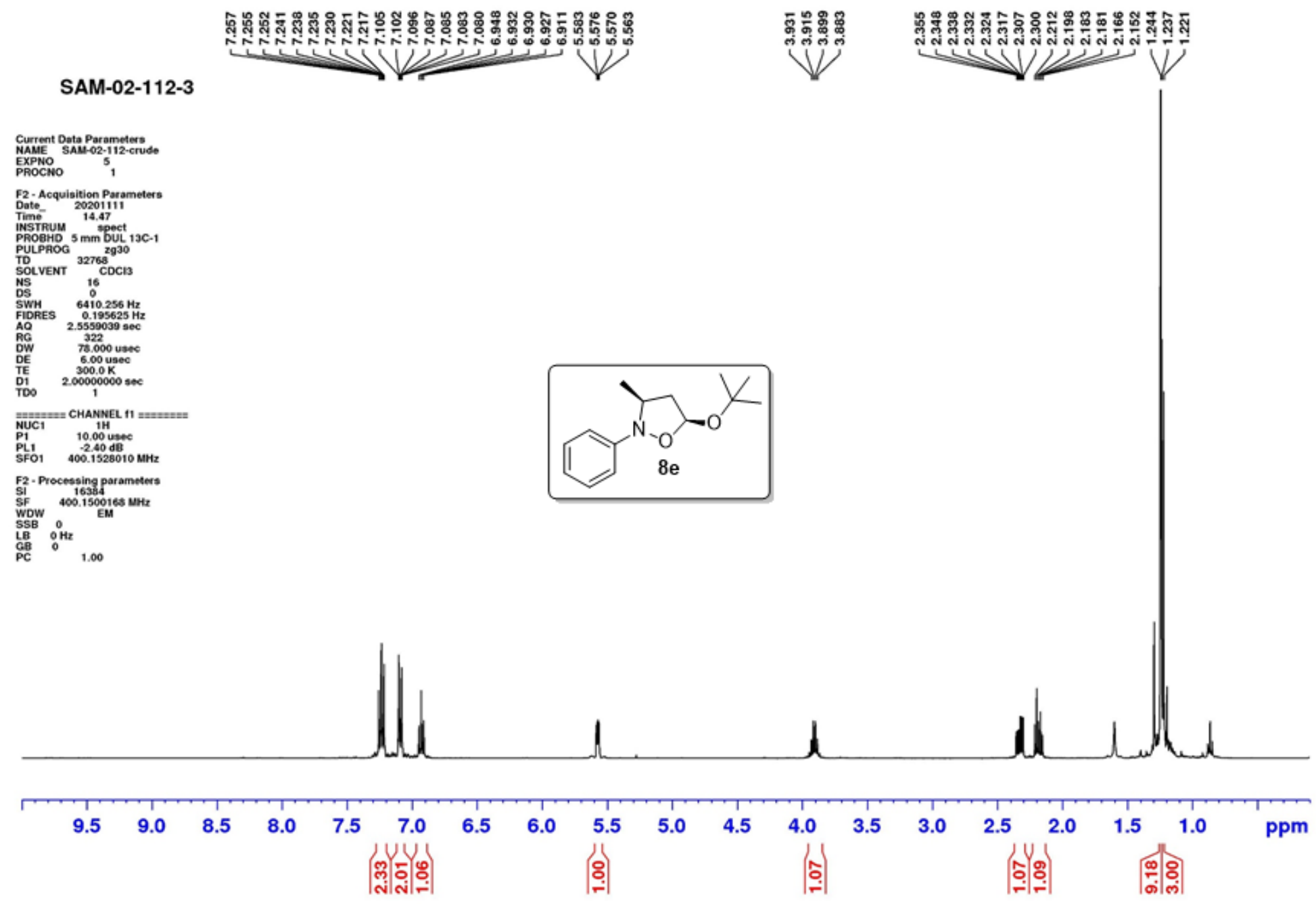


Solvent: $\mathrm{CDCl}_{3}$

SFO1: $100 \mathrm{MHz}$

SAM-02-112-C

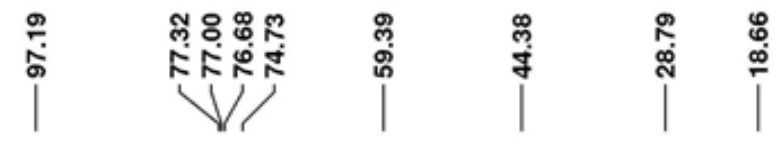

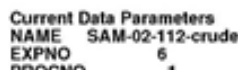

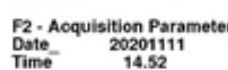

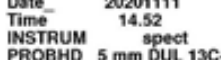

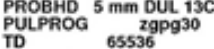

TSOLVENT ${ }^{65536}{ }_{\text {COCII }}$

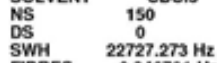

ADRES $\begin{gathered}0.3467901 \mathrm{~Hz} \\ \text { A. }\end{gathered}$

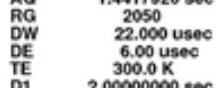

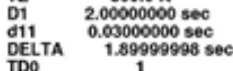

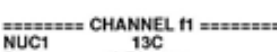

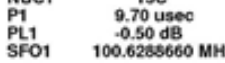

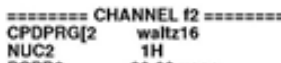

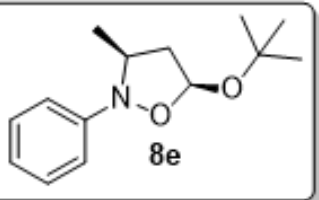

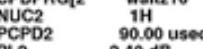

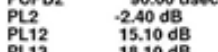

SFO2 400.1516010 MHE

Fi2. Procesessing porameters

SIF
ST
WOW

$\underset{L B}{5 S B} 3.00 \mathrm{~Hz}$

GB
PC

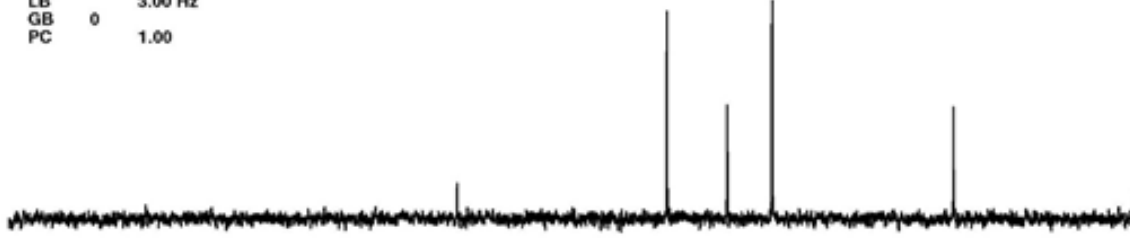


Solvent: $\mathrm{CDCl}_{3}$ SFO1: $700 \mathrm{MHz}$

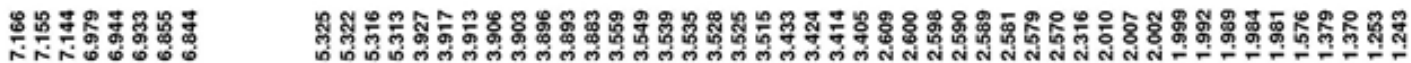

SAM-02-128-1

Current Data Parameters

$\begin{array}{ll}\text { NAME } & 1 \\ \text { EXPNO } & 999 \\ \text { PROCNO } & 1\end{array}$

F2 - Processing parameters

SI $\quad \begin{array}{ll}65536 \\ \text { SF }\end{array}$

WDW

$\begin{array}{lll}\mathrm{LB} & 0 & 0.30 \mathrm{~Hz} \\ \mathrm{~GB} & 0 & 1.00\end{array}$

$\begin{array}{lll}\mathrm{PC} & 1.00\end{array}$

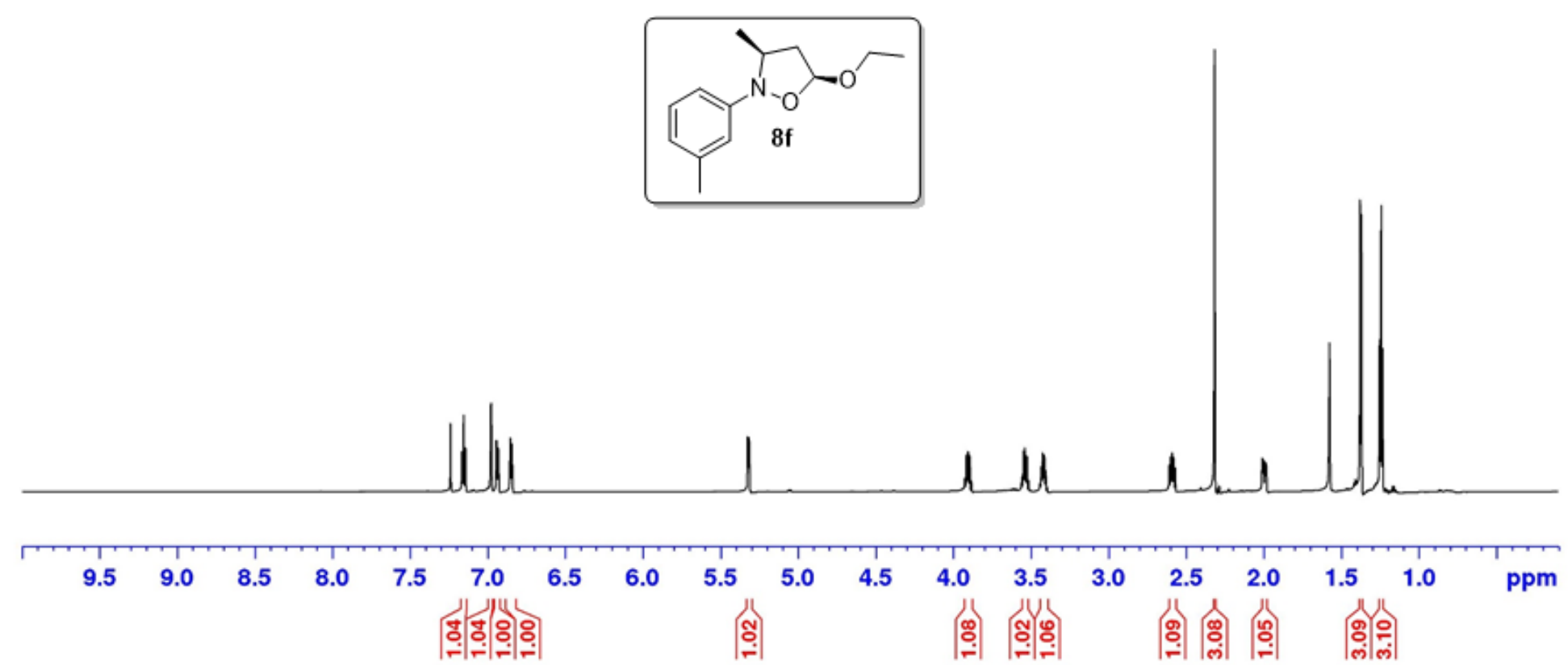


Solvent: $\mathrm{CDCl}_{3}$

SFO1: $175 \mathrm{MHz}$

SAM-02-128-1-C

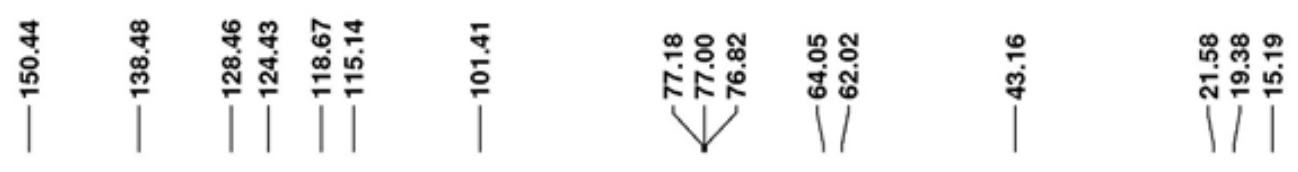

Current Data Parameters

$\begin{array}{lc}\text { NAME } & 3 \\ \text { EXPNO } & 999\end{array}$

EXPNO

F2 - Processing parameters

$\mathrm{SI}$

$\begin{array}{lc}\text { SF } & 175.9505398 \mathrm{MHz} \\ \text { WDW } & \text { EM }\end{array}$

SSB 0 EM

$\begin{array}{lll}\text { LB } & 0.30 \\ \text { GB } & 0 & \end{array}$

PC $\quad \mathbf{1 . 0 0}$
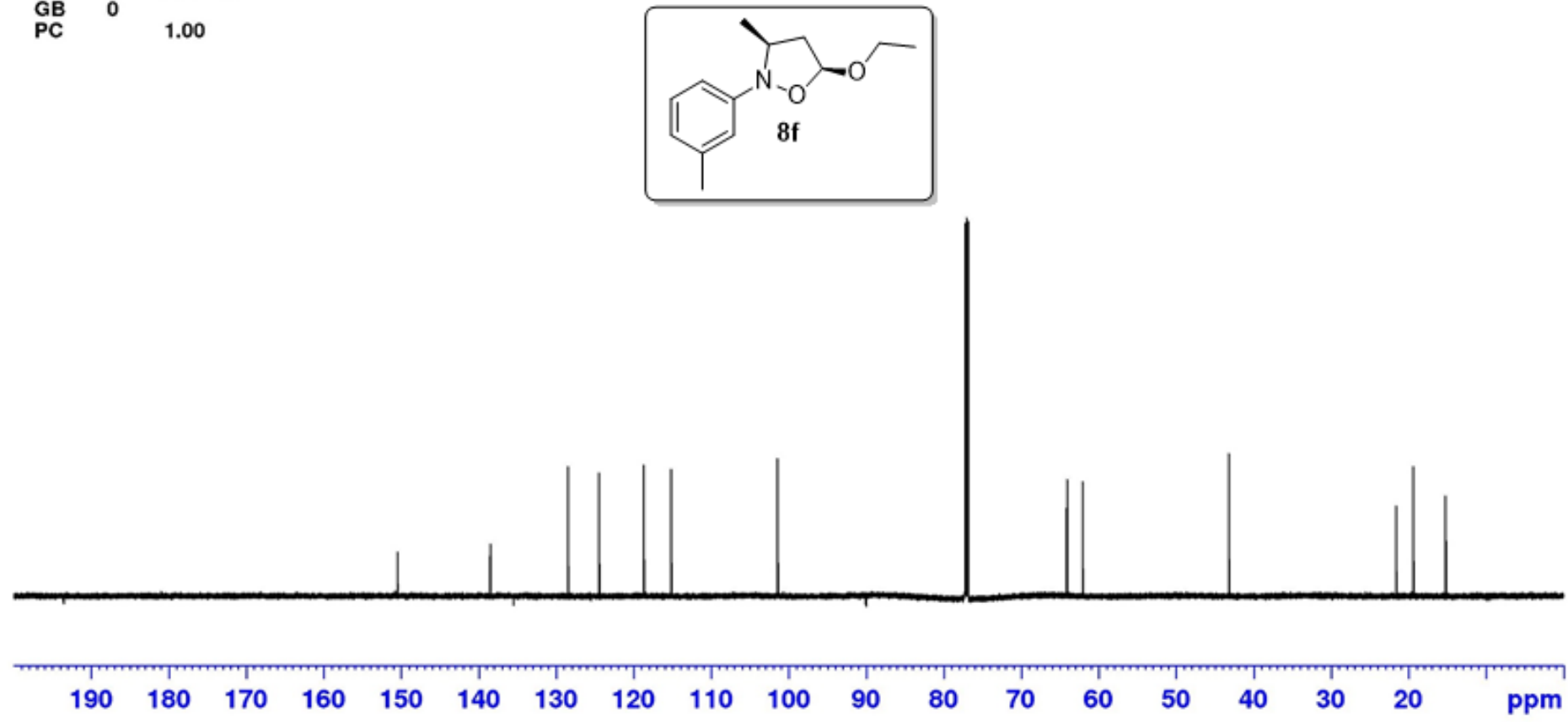
Solvent: $\mathrm{CDCl}_{3}$ SFO1: $700 \mathrm{MHz}$

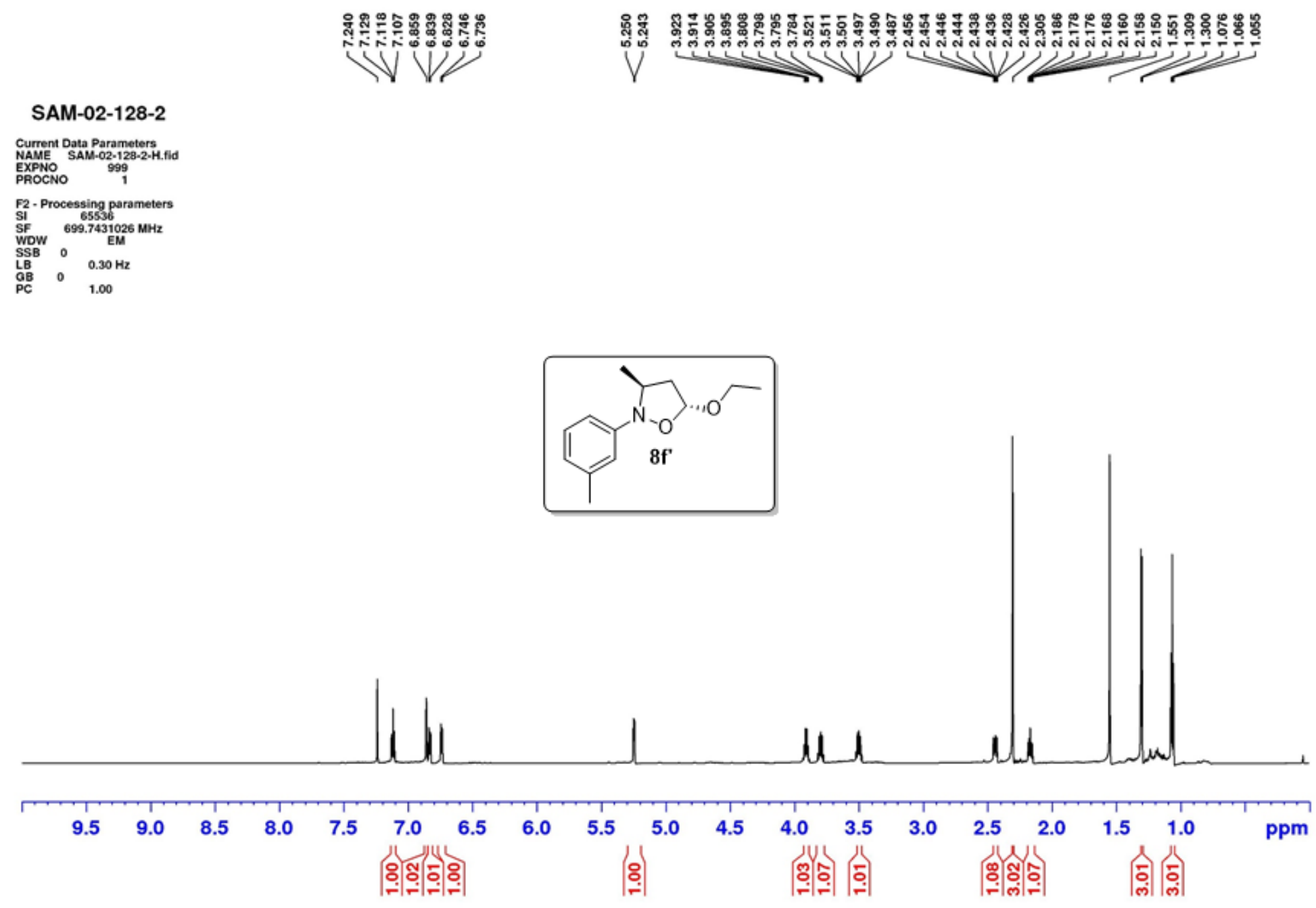


Solvent: $\mathrm{CDCl}_{3}$

SFO1: $175 \mathrm{MHz}$

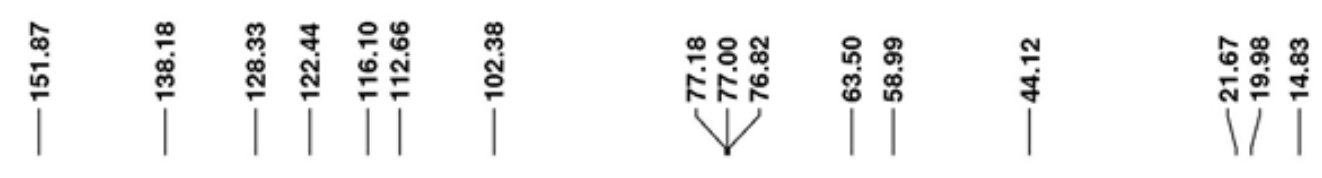

SAM-02-128-2-C

Current Data Parameters

NAME

EXPNO

F2 - Processing parameters

$\begin{array}{ll}\text { SI } & 131072 \\ \text { SF } & 175.9505398 \\ \text { MHz }\end{array}$

WDW EM

$\begin{array}{lll}\text { SSB } & 0 & \\ \text { LB } & 0.30 \mathrm{~Hz}\end{array}$

$\begin{array}{lll}\text { GB } & 0 & 0.30 \\ \text { PC } & & 1.00\end{array}$
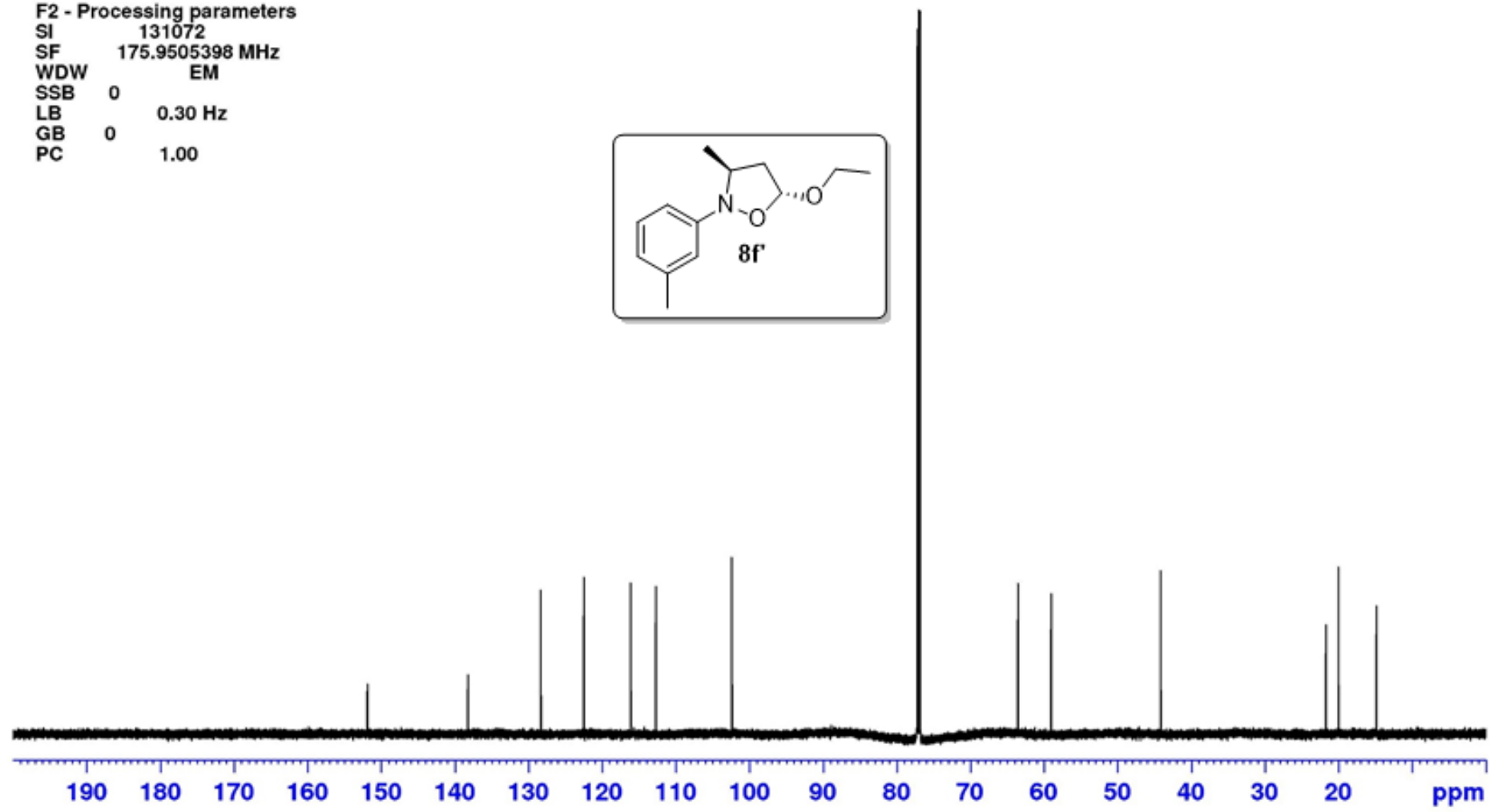
Solvent: $\mathrm{CDCl}_{3}$ SFO1: $500 \mathrm{MHz}$

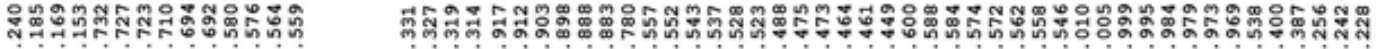
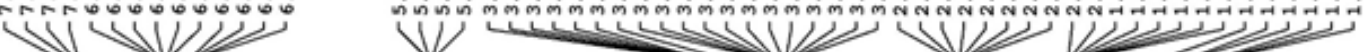

\section{SAM-3-17-A-1}

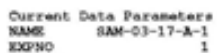

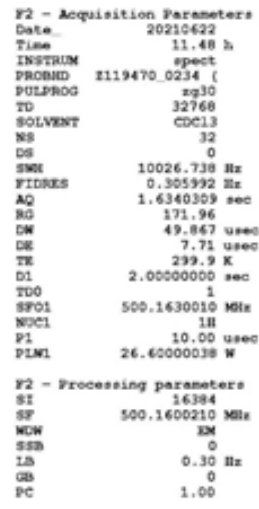
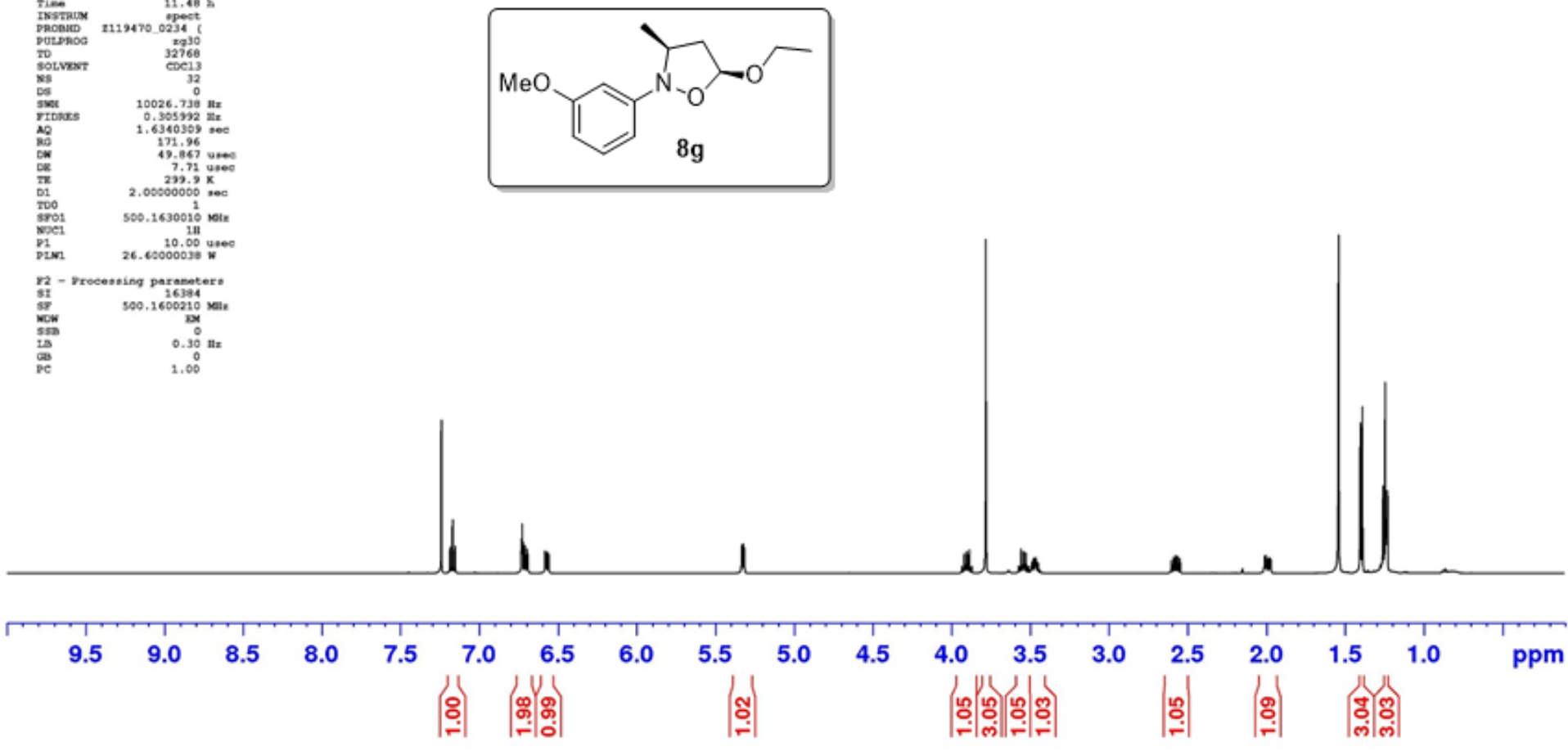
Solvent: $\mathrm{CDCl}_{3}$ SFO1: $125 \mathrm{MHz}$

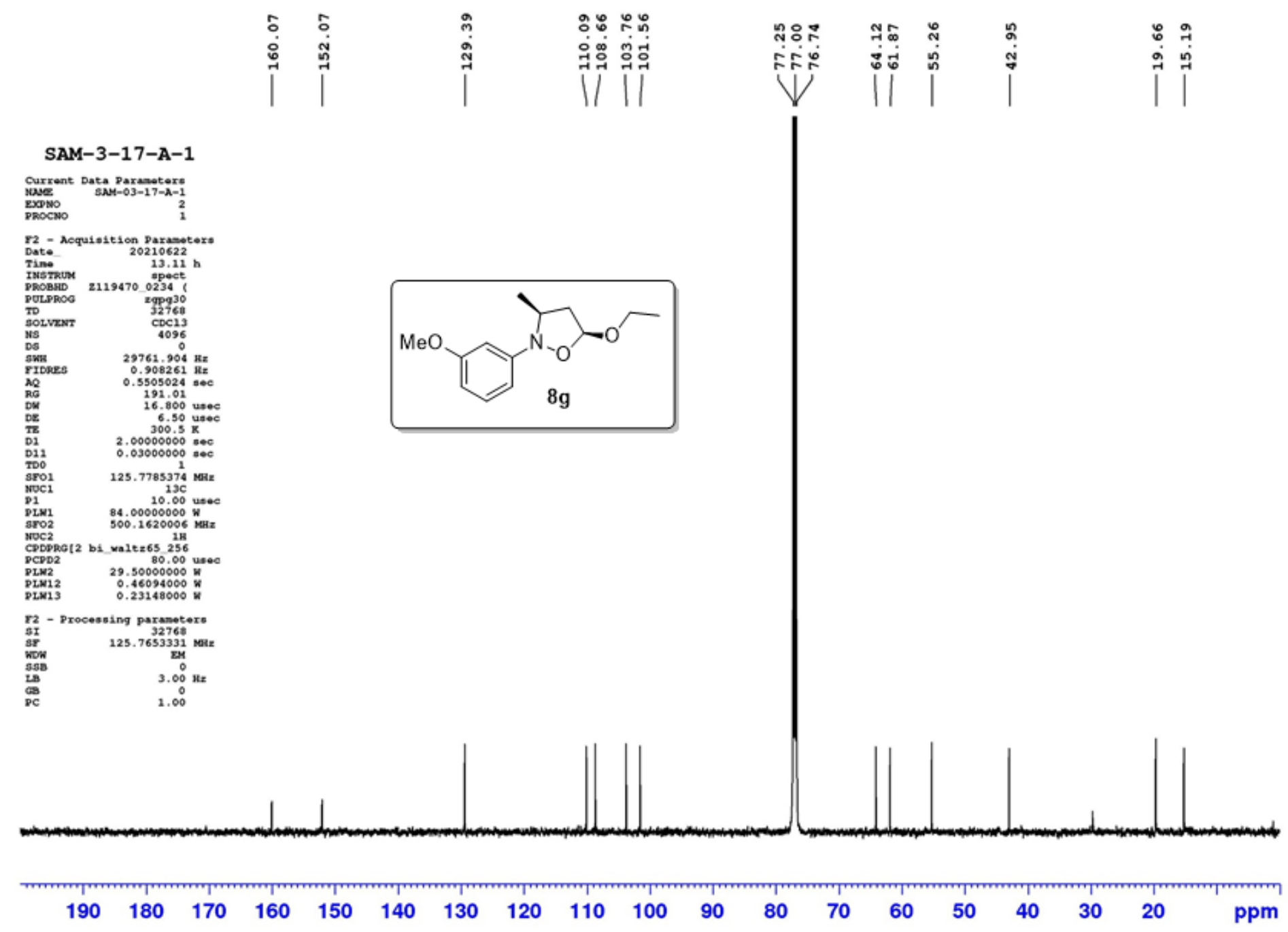


Solvent: $\mathrm{CDCl}_{3}$ SFO1: $400 \mathrm{MHz}$

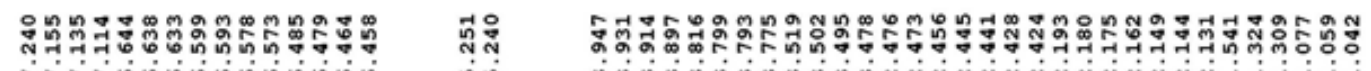

(

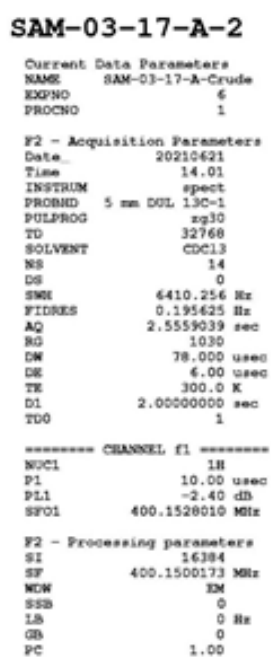

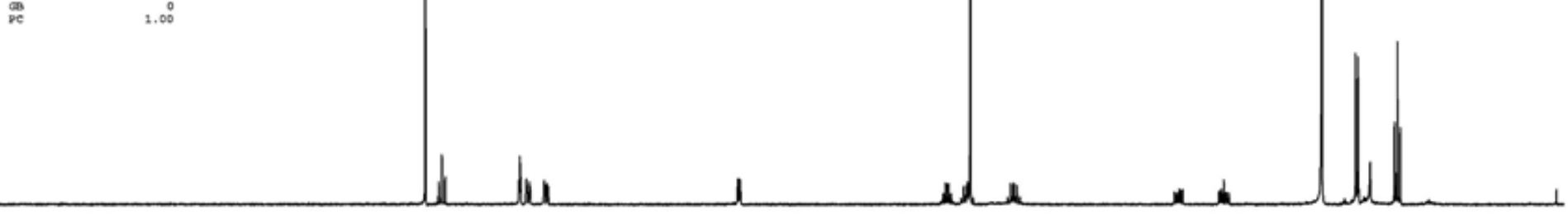

$\begin{array}{lllll}9.5 & 9.0 & 8.5 & 8.0 & 7.5\end{array}$

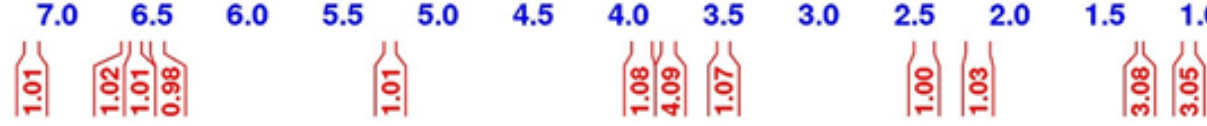


Solvent: $\mathrm{CDCl}_{3}$

SFO1: $100 \mathrm{MHz}$

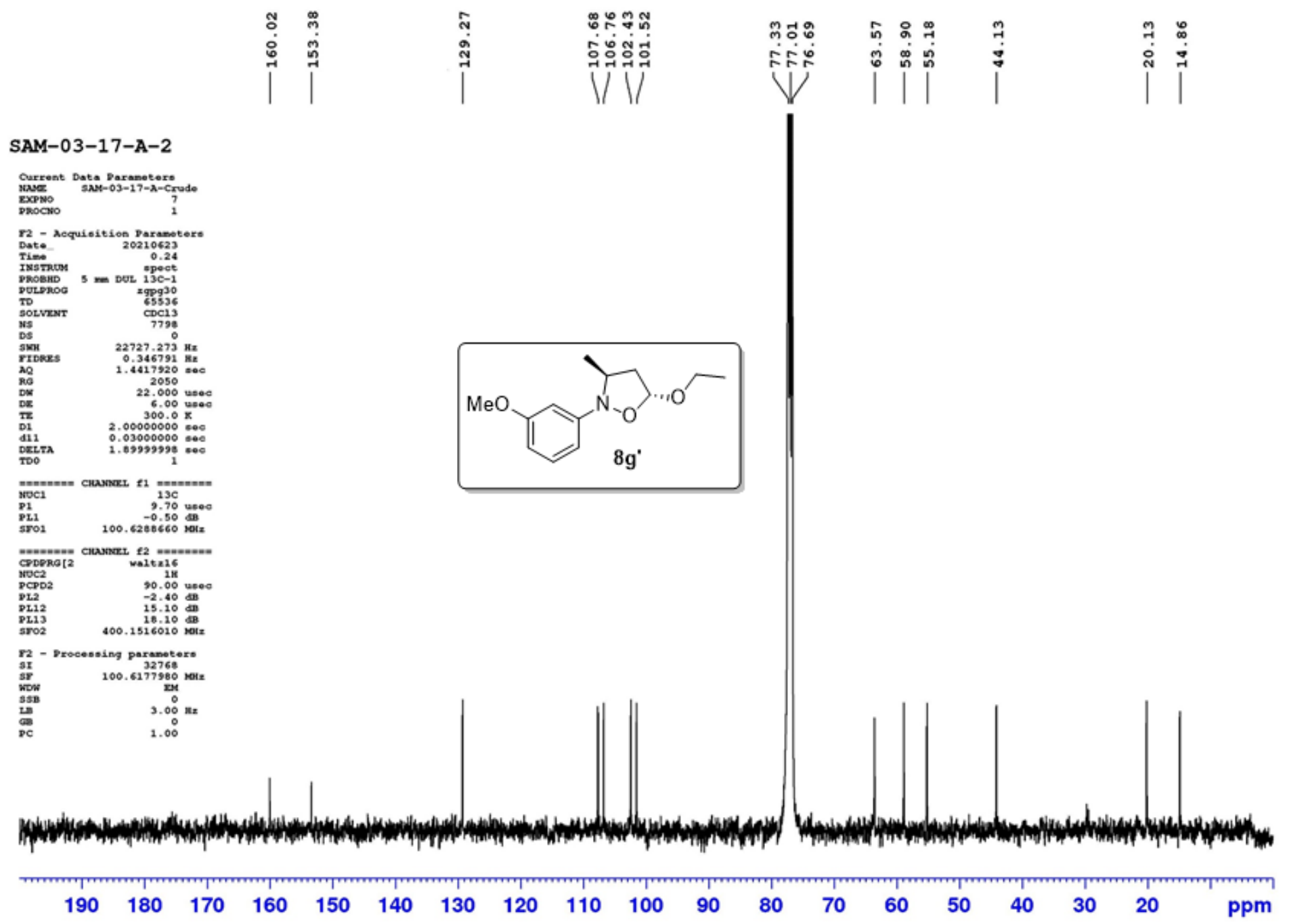


Solvent: $\mathrm{CDCl}_{3}$ SFO1: $700 \mathrm{MHz}$

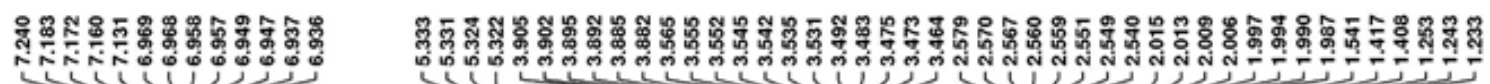

;AM-02-131-:

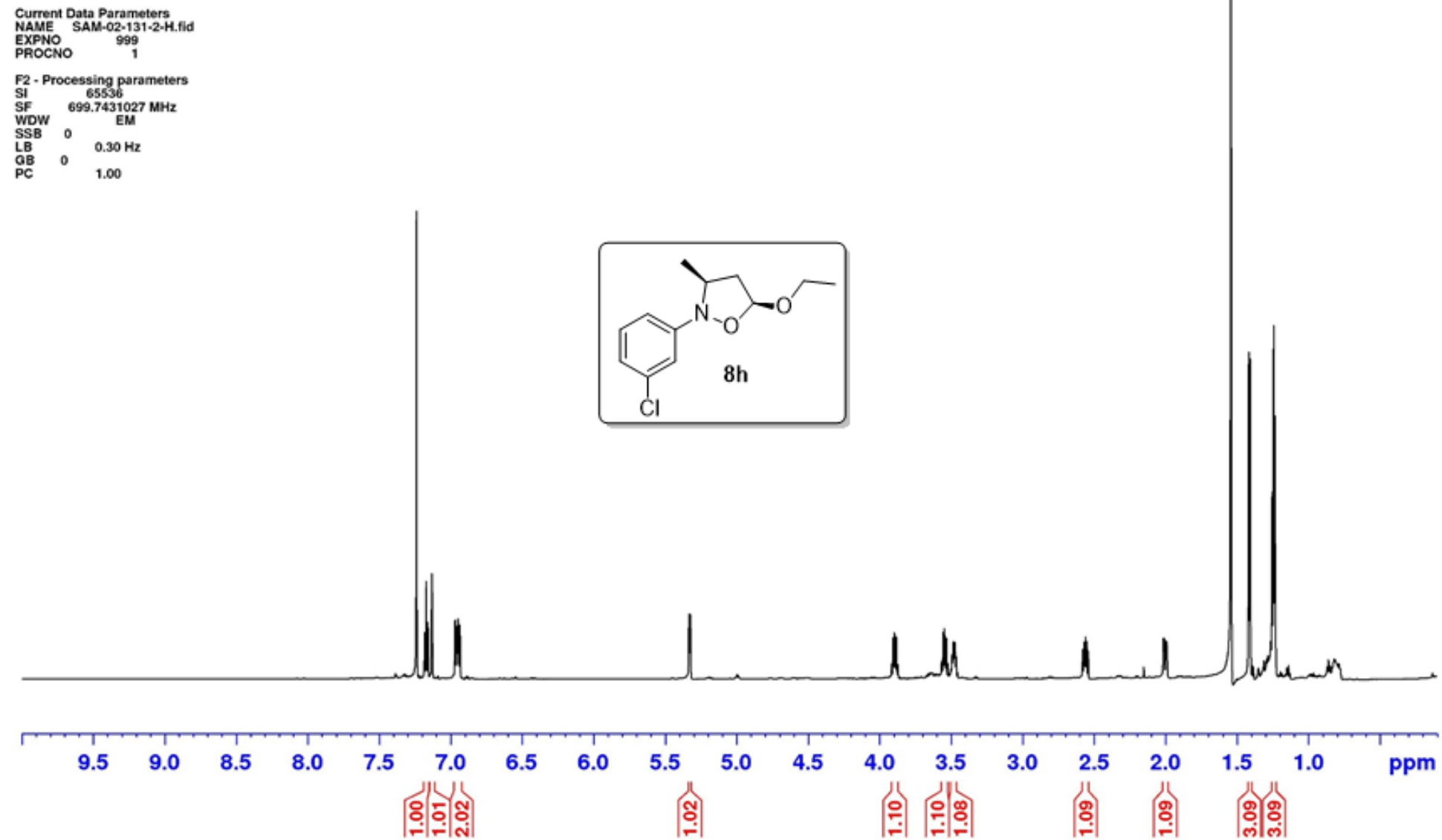


Solvent: $\mathrm{CDCl}_{3}$

SFO1: $175 \mathrm{MHz}$
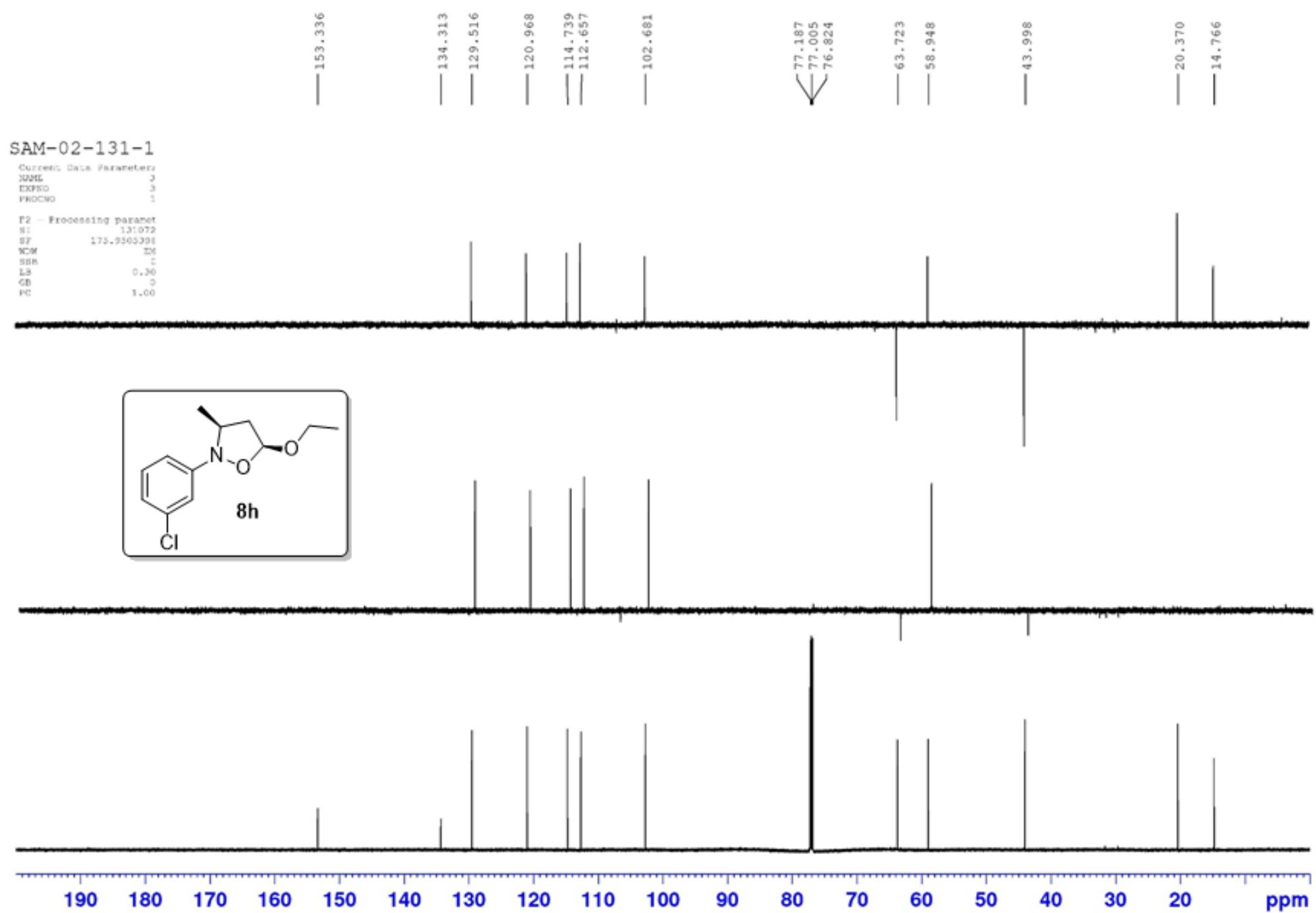
Solvent: $\mathrm{CDCl}_{3}$

SFO1: $700 \mathrm{MHz}$

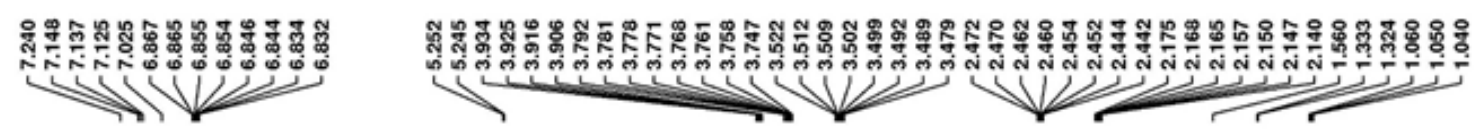

;AM-02-131-

Current Data Parameters

EXPNO
PROCNO

F2. Procossing parameters

$\begin{array}{ll}\text { SI } & 65536 \\ \text { SF } & 69.7431020 \\ \text { MHz }\end{array}$

WDW

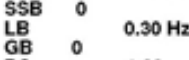

PC 1.00

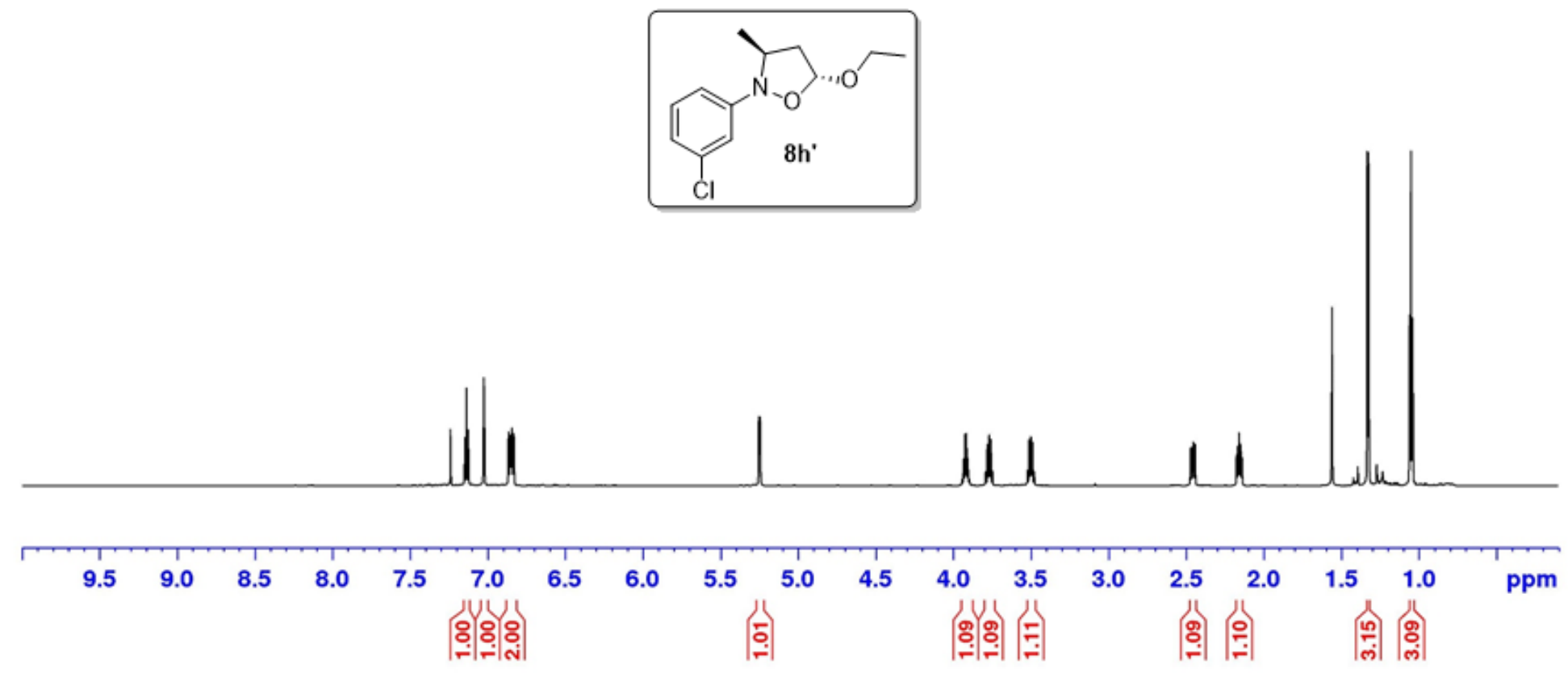


Solvent: $\mathrm{CDCl}_{3}$ SFO1: $175 \mathrm{MHz}$

SAM-02-131-2-C

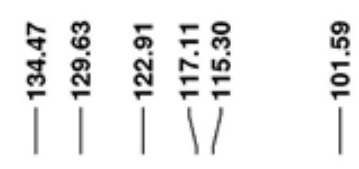

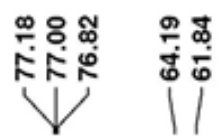

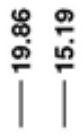

Current Data Parameters

$\begin{array}{ll}\text { NAME } & 3 \\ \text { EXPNO } & 999\end{array}$

F2 - Processing parameters

$\begin{array}{ll}\text { SI } & 131072 \\ \text { SF } & 175.9505398 \mathrm{MHz}\end{array}$

WDW 175.9505398

$\begin{array}{lll}\text { SSB } & 0 & 0.30 \mathrm{~Hz}\end{array}$

$\begin{array}{ll}\text { LB } & 0.30 \\ \text { GB } & \end{array}$

PC

1.00

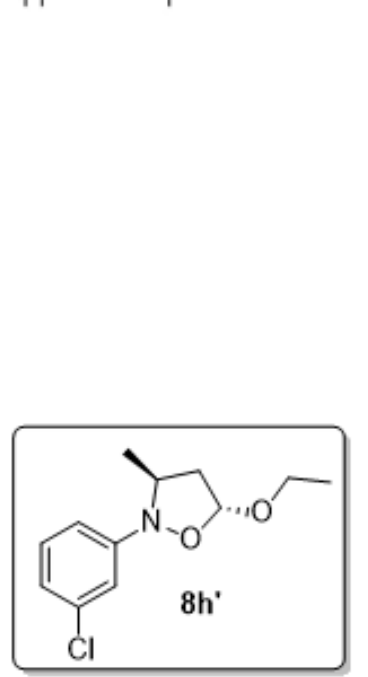

ํํำ

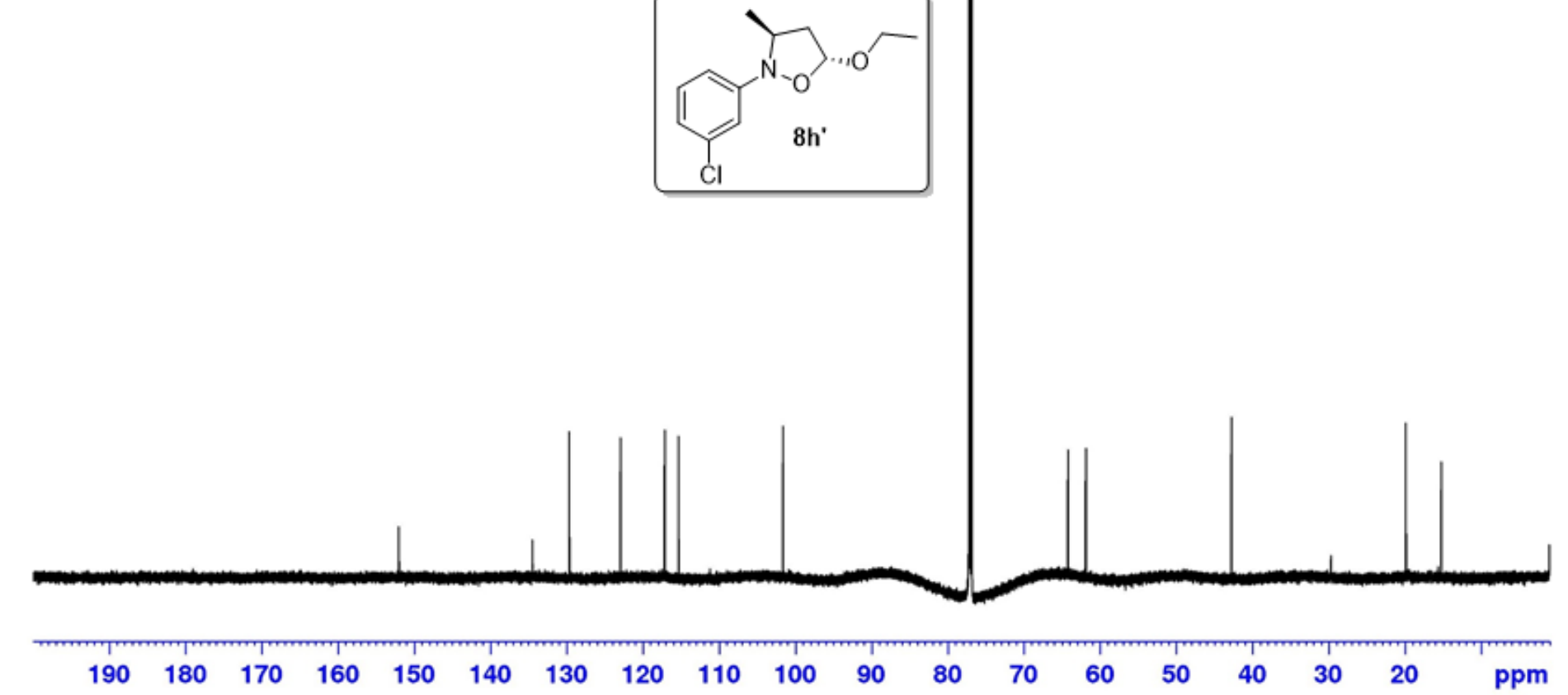


Solvent: $\mathrm{CDCl}_{3}$ SFO1: $700 \mathrm{MHz}$

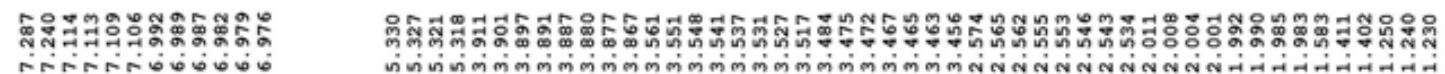

N V V

SAM-02-132-1

current Data Paramater.

Drocio

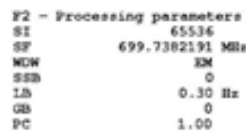
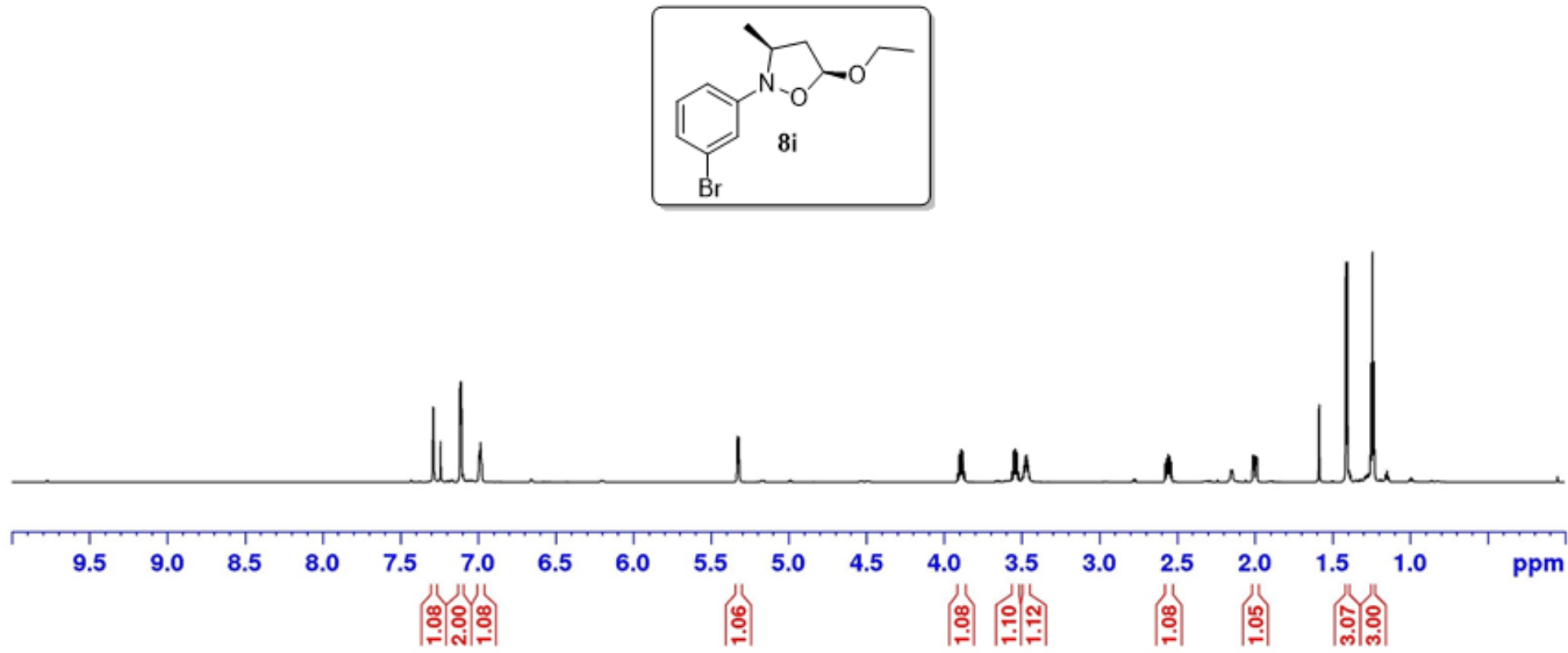
Solvent: $\mathrm{CDCl}_{3}$

SFO1: $175 \mathrm{MHz}$

SAM-02-132-1 |

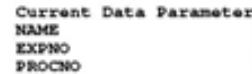

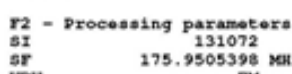
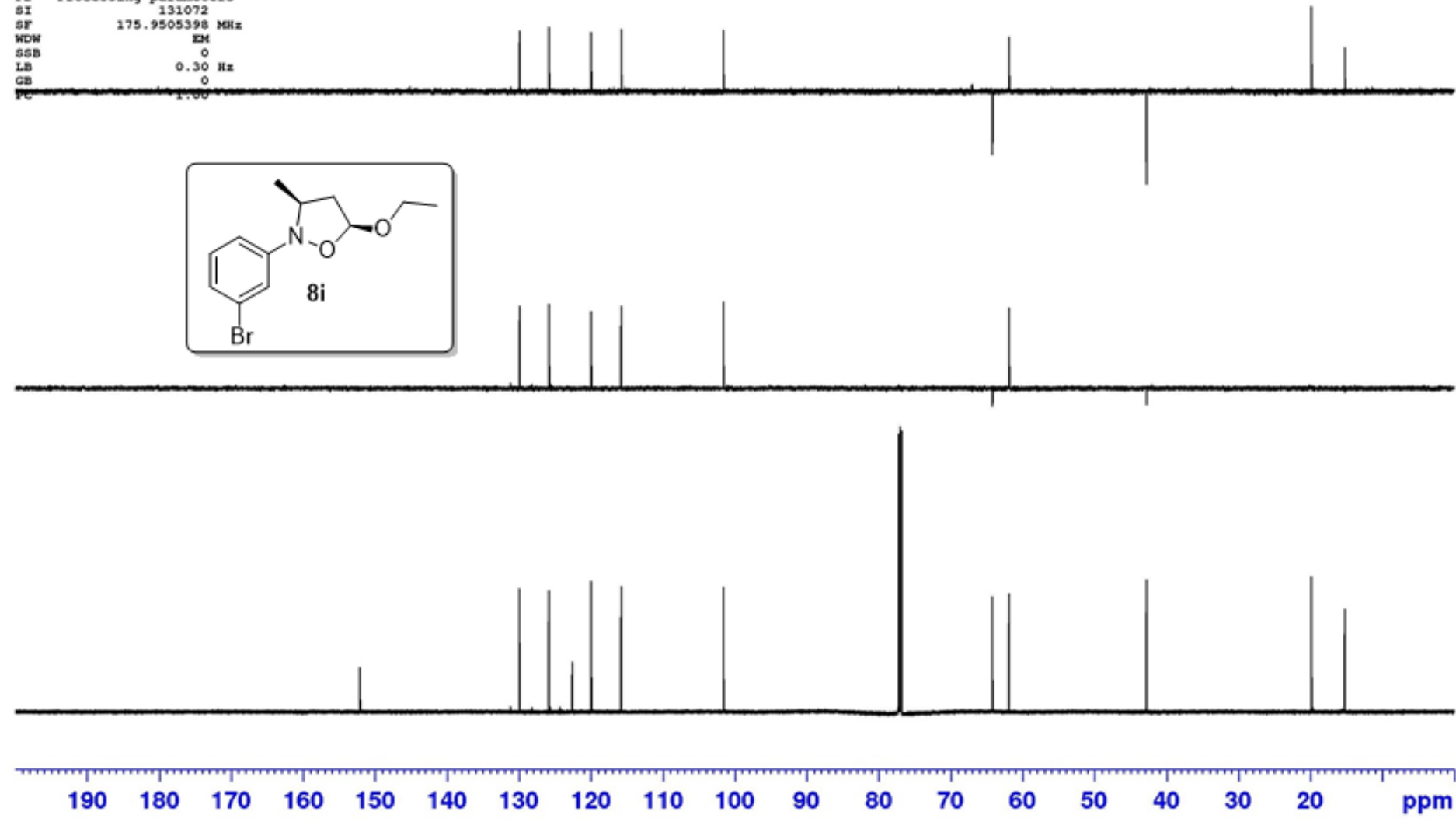
Solvent: $\mathrm{CDCl}_{3}$ SFO1: $700 \mathrm{MHz}$

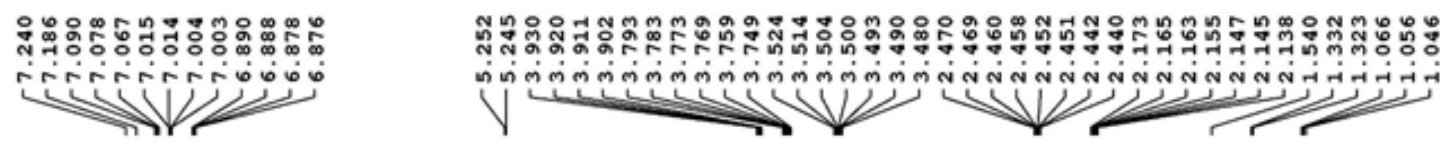

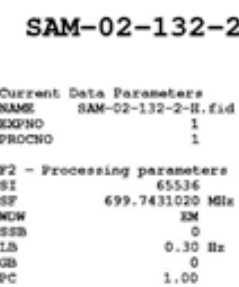

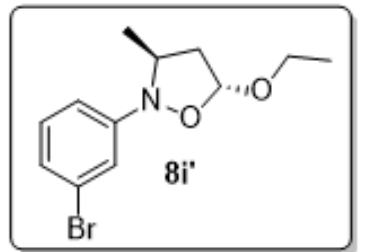

(1)

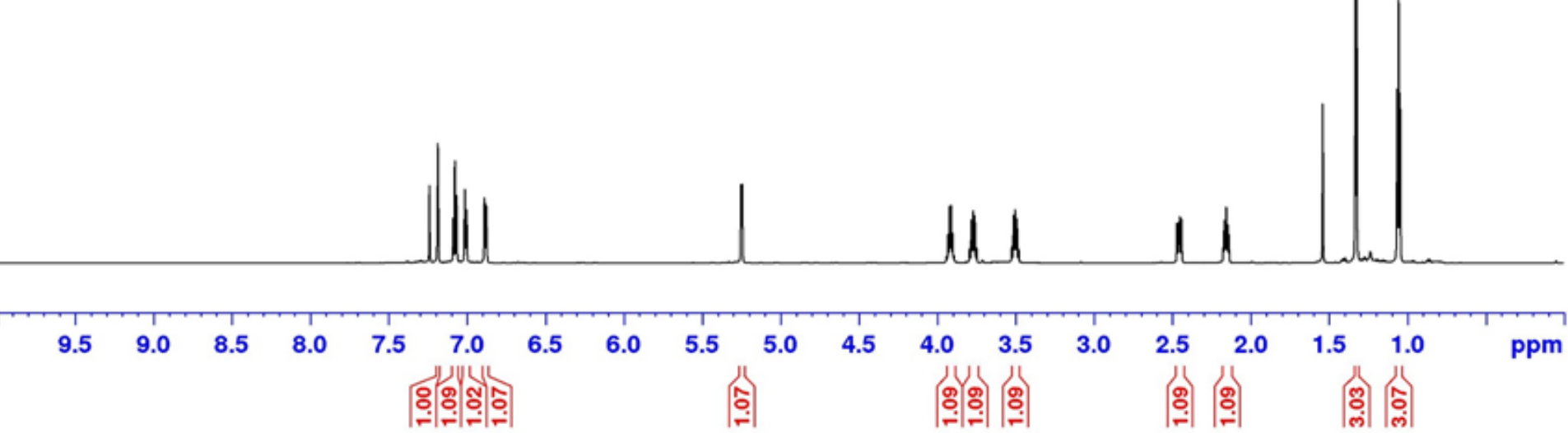


Solvent: $\mathrm{CDCl}_{3}$

SFO1: $175 \mathrm{MHz}$
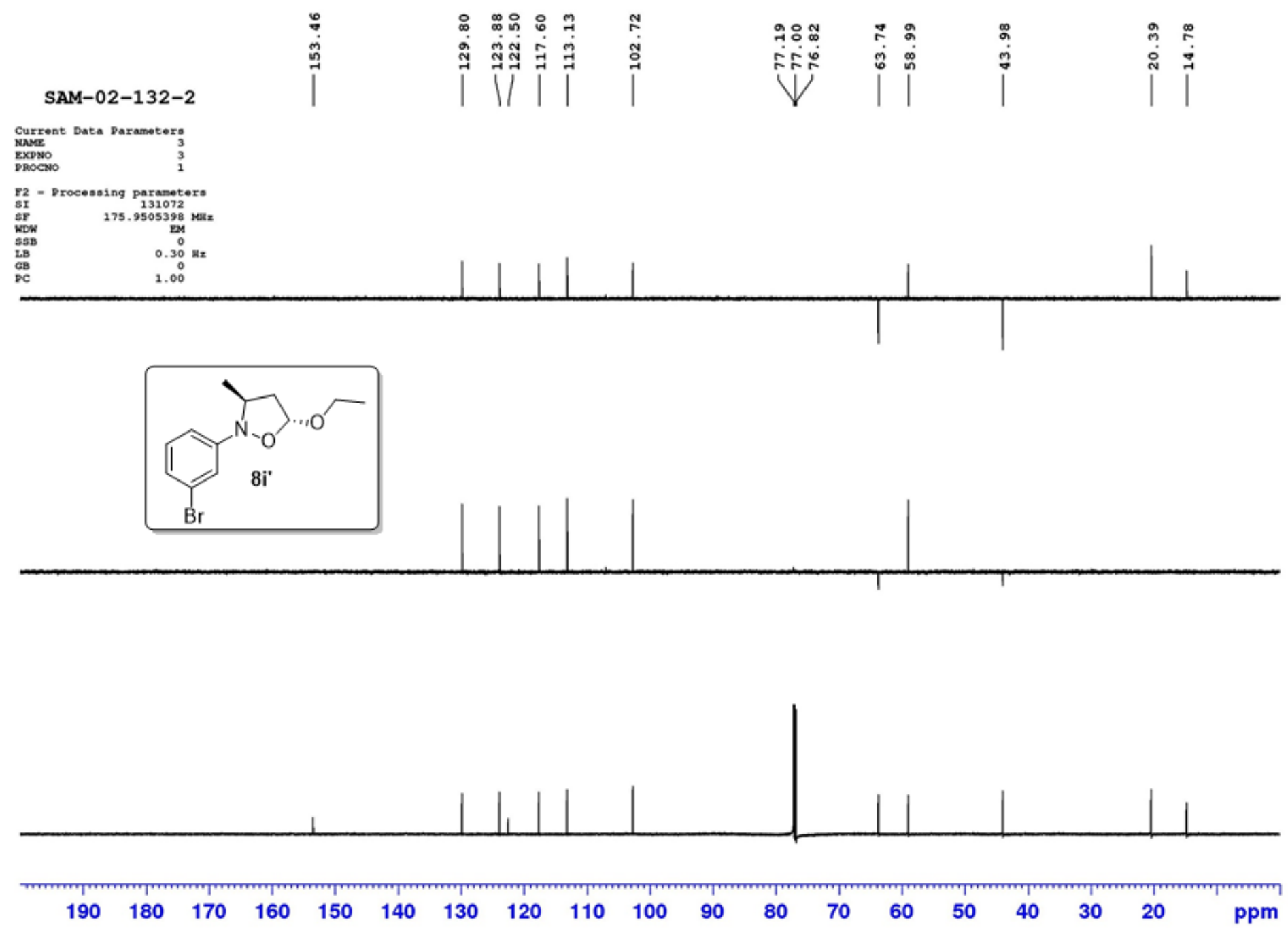
Solvent: $\mathrm{CDCl}_{3}$ SFO1: $700 \mathrm{MHz}$

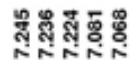

VंV

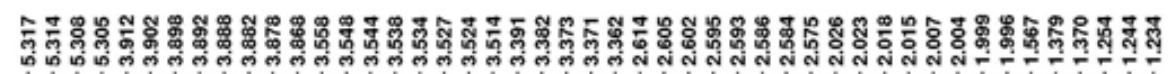

过

;AM-02-113-

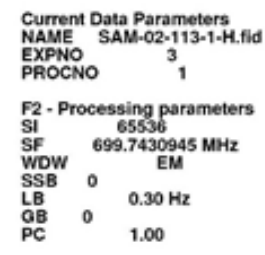

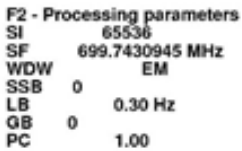

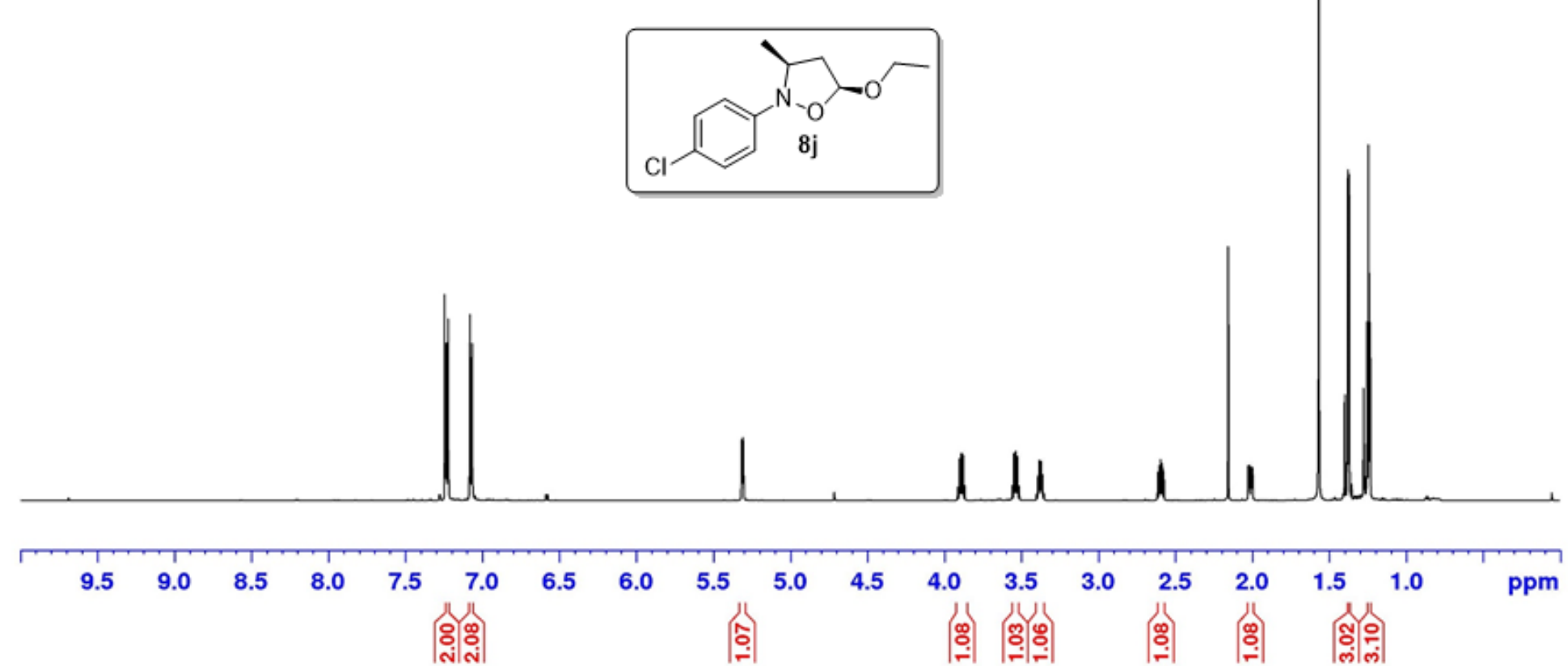


Solvent: $\mathrm{CDCl}_{3}$

SFO1: $175 \mathrm{MHz}$
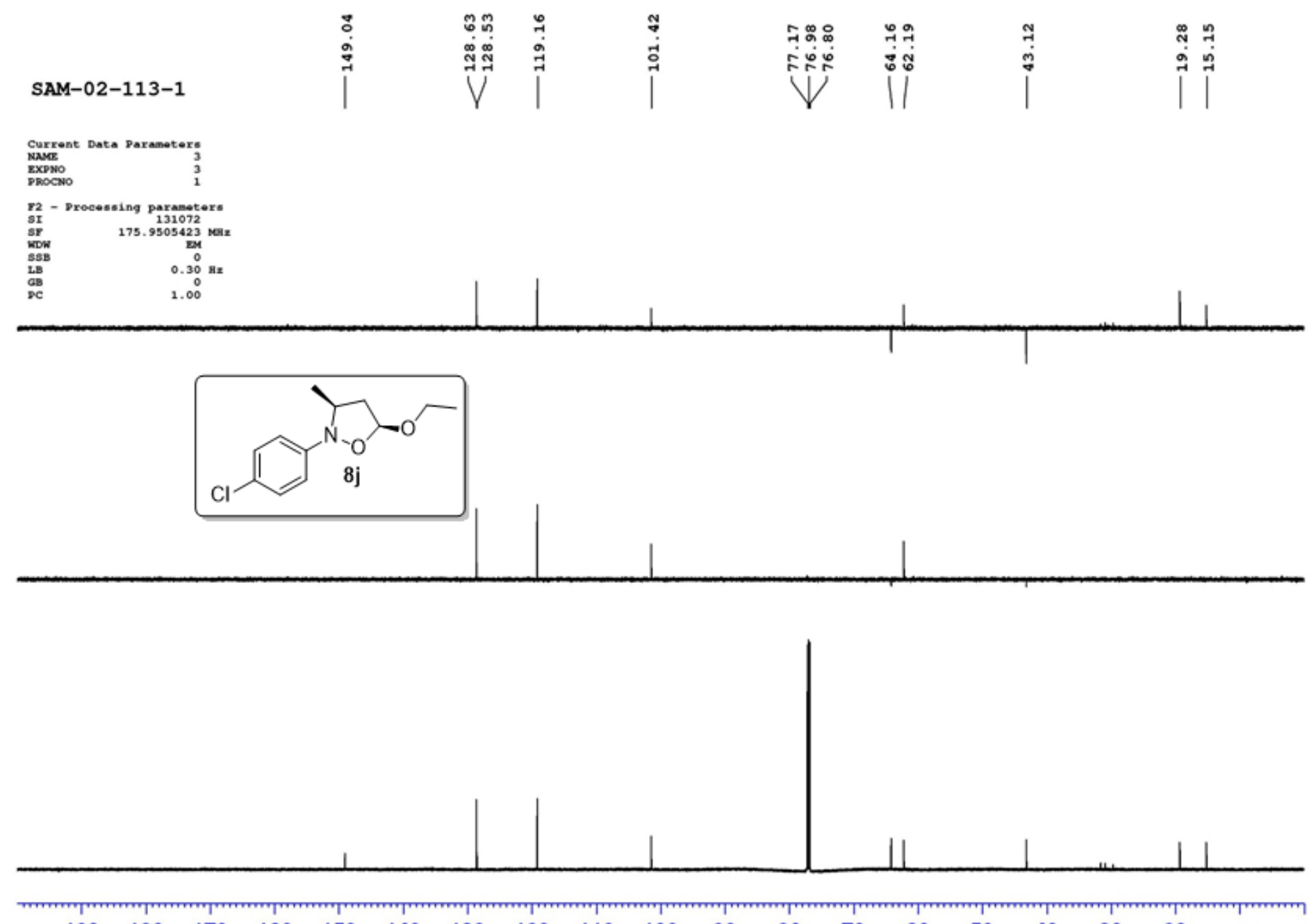

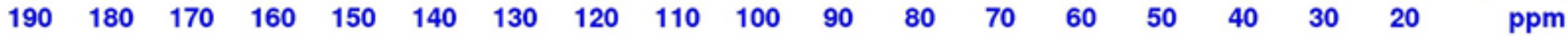


Solvent: $\mathrm{CDCl}_{3}$ SFO1: $700 \mathrm{MHz}$

กติ

iij

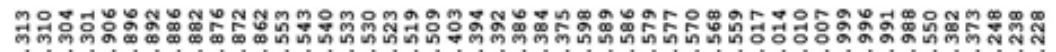

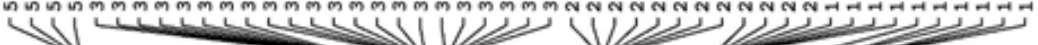

SAM-02-125-1

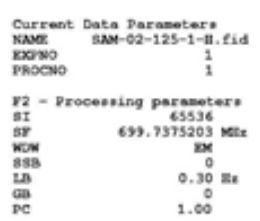
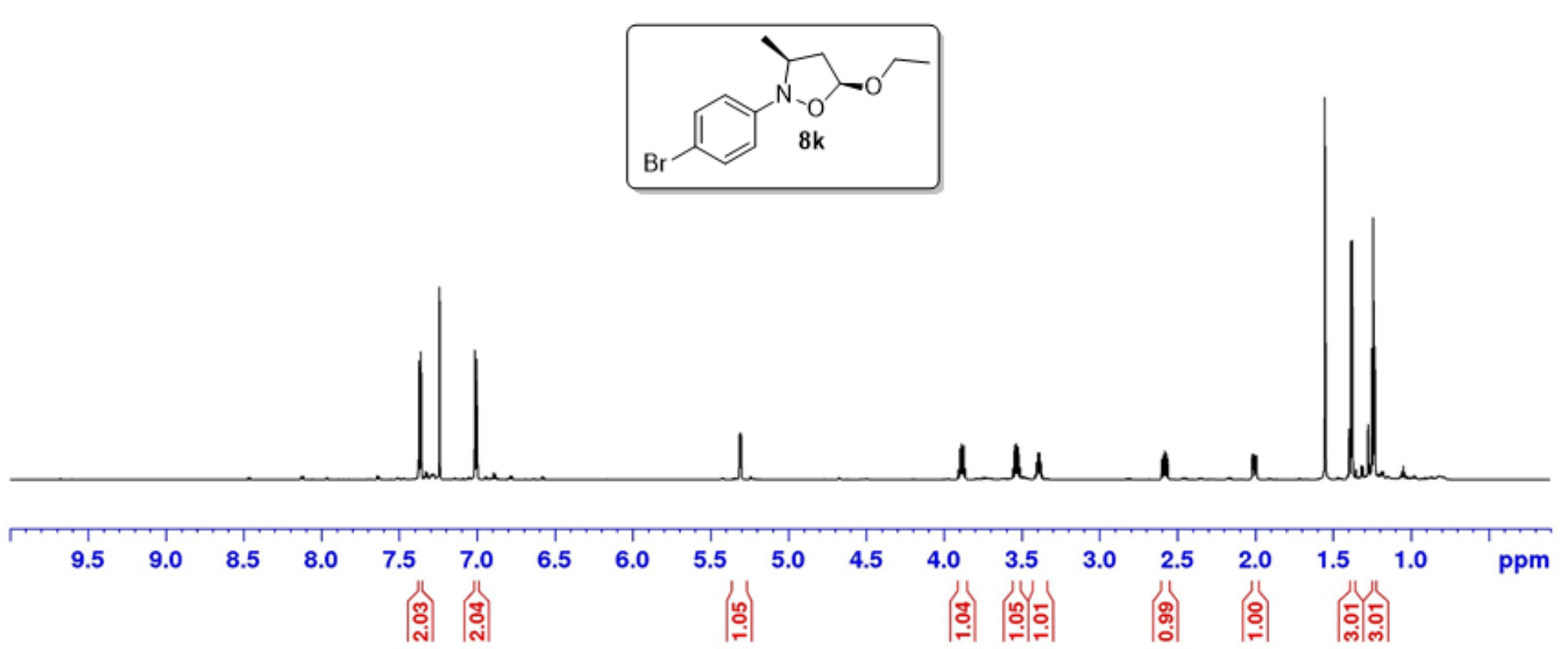
Solvent: $\mathrm{CDCl}_{3}$

SFO1: $175 \mathrm{MHz}$

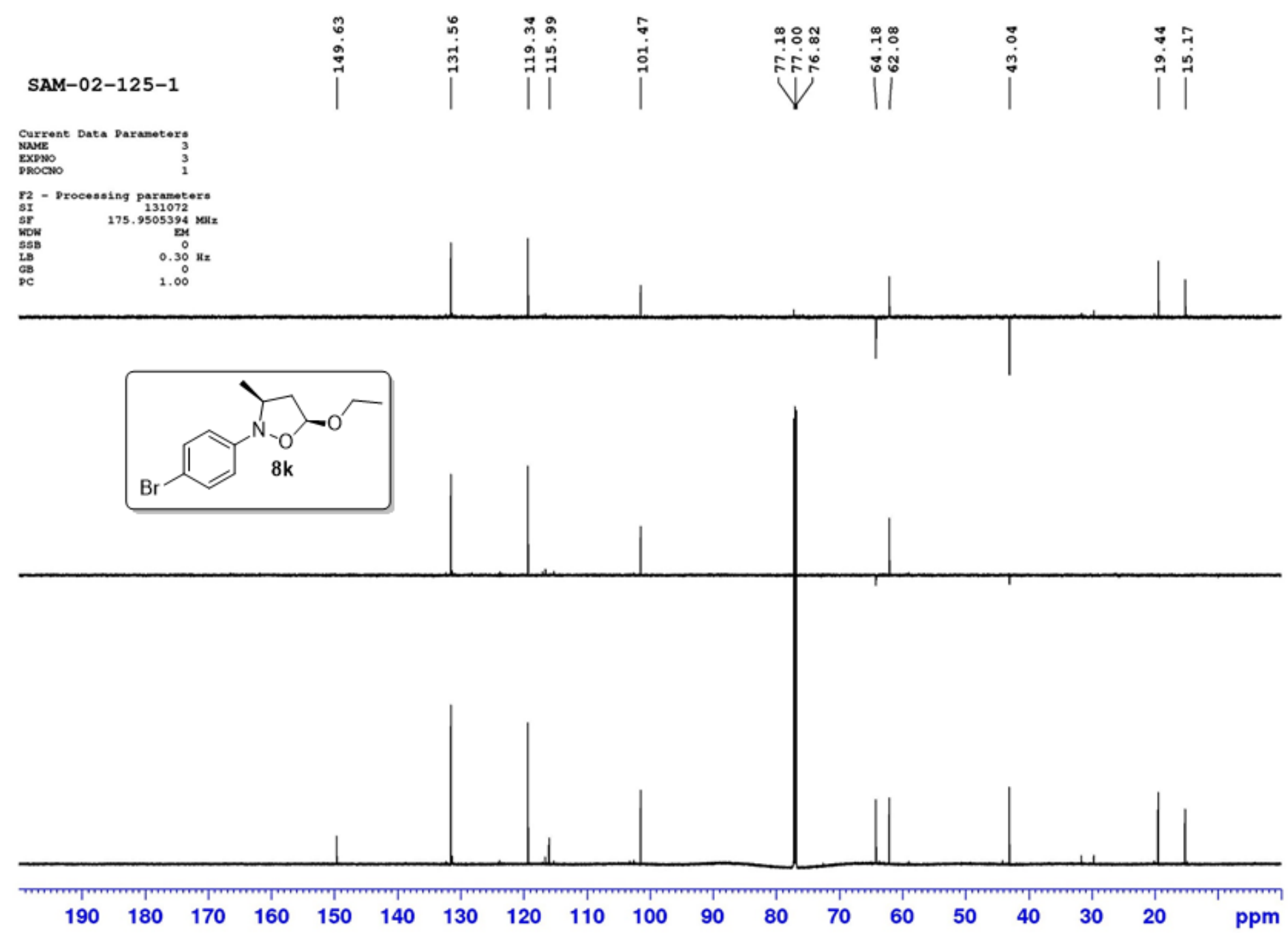


Solvent: $\mathrm{CDCl}_{3}$ SFO1: $700 \mathrm{MHz}$

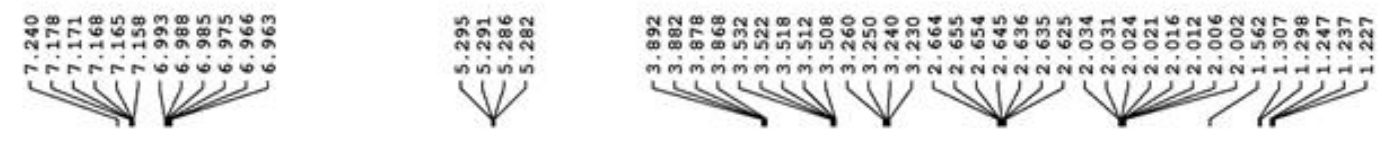

SAM-02-126-1

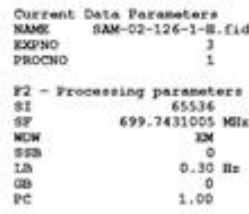
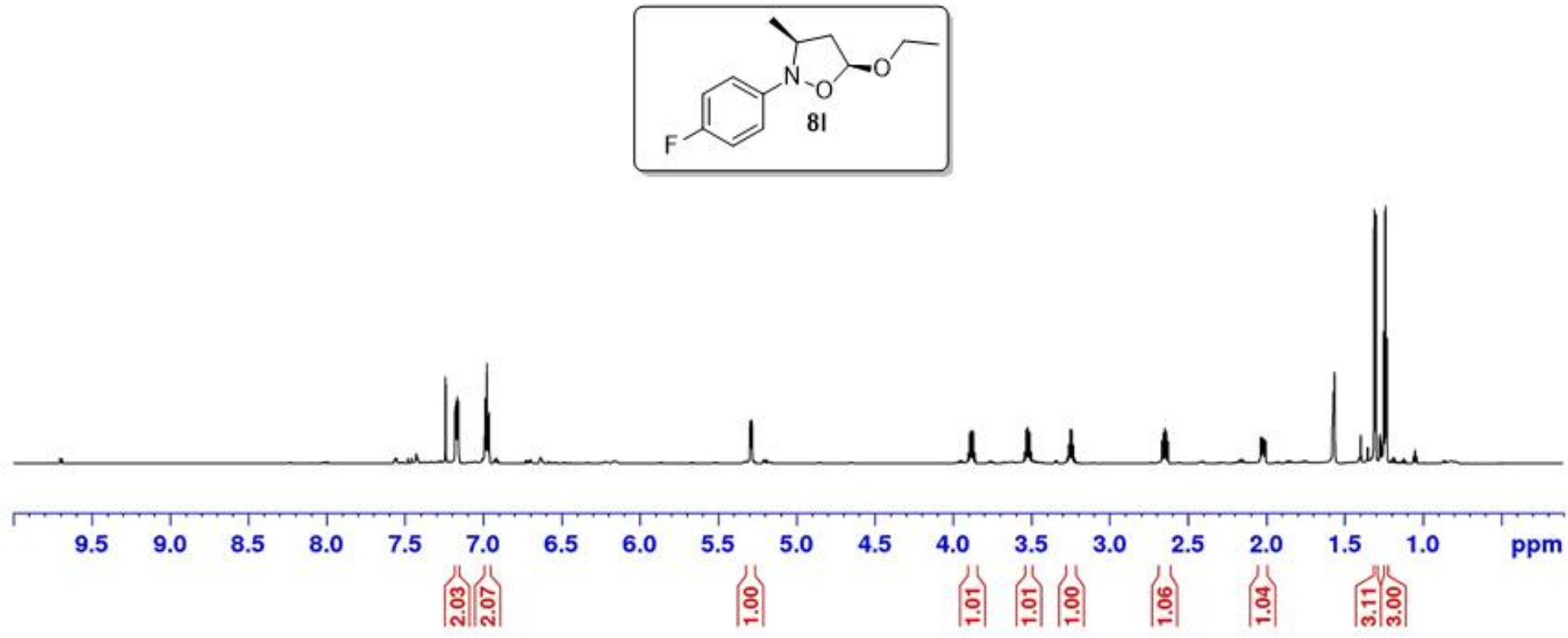
Solvent: $\mathrm{CDCl}_{3}$

SFO1: $175 \mathrm{MHz}$
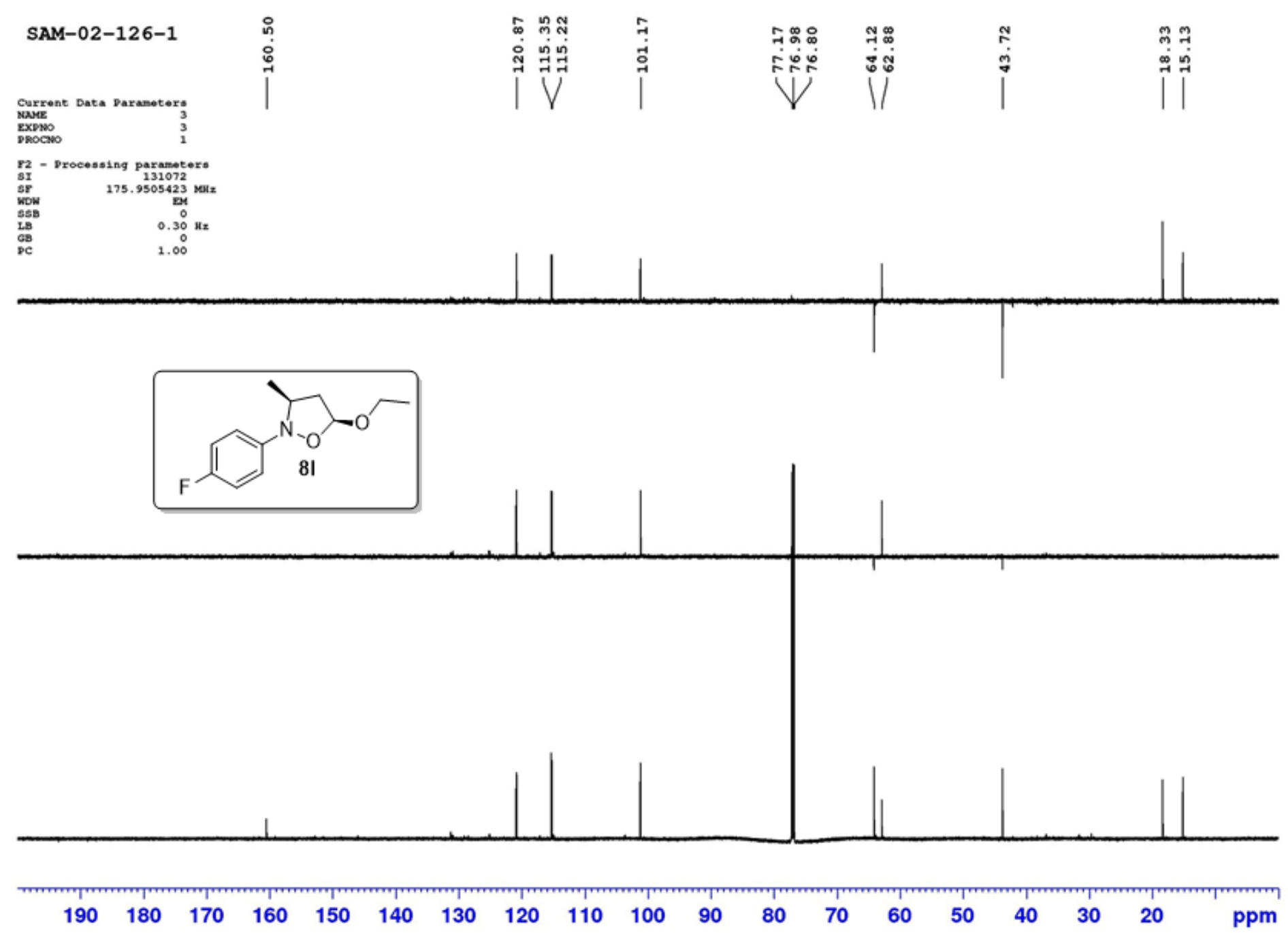
Solvent: $\mathrm{CDCl}_{3}$ SFO1: $700 \mathrm{MHz}$

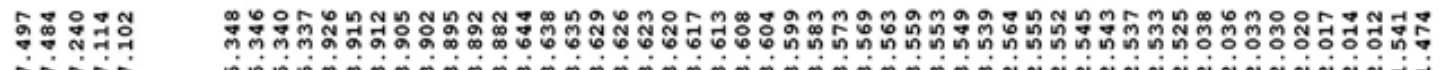

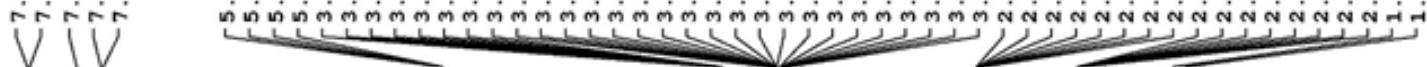

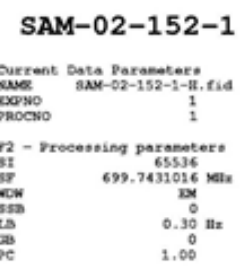
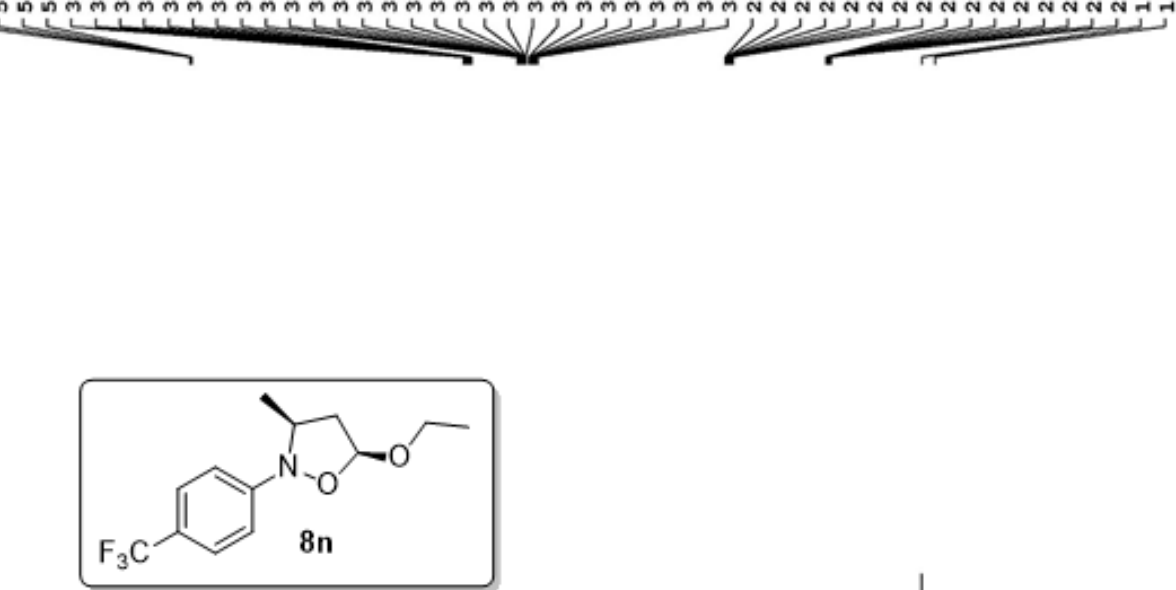

$$
\text { J }
$$
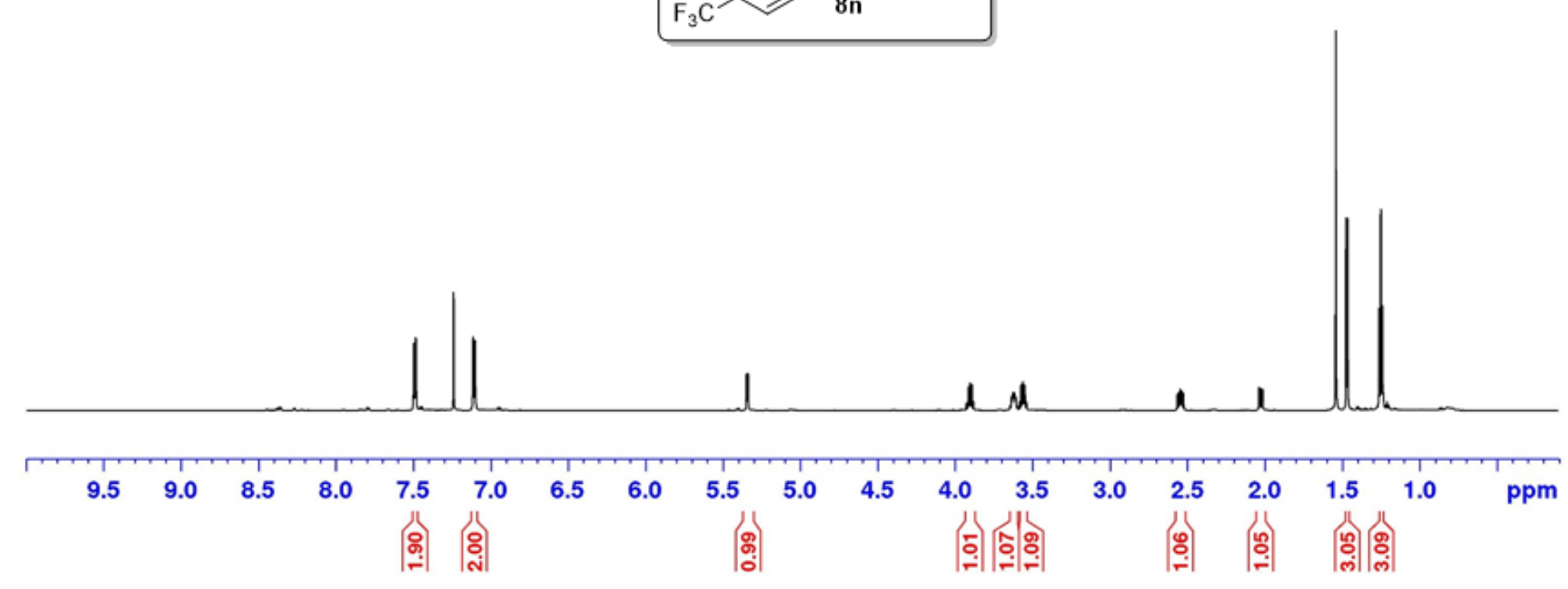
Solvent: $\mathrm{CDCl}_{3}$

SFO1: $175 \mathrm{MHz}$

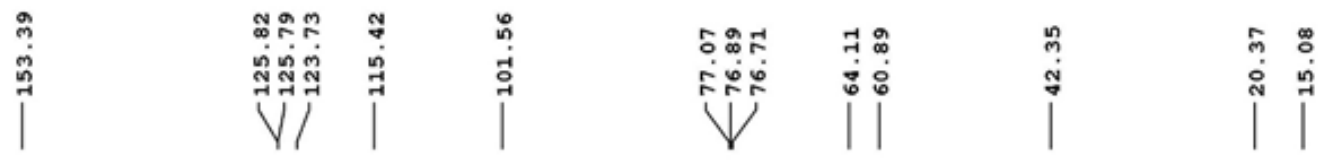

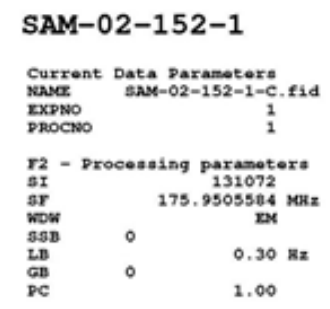
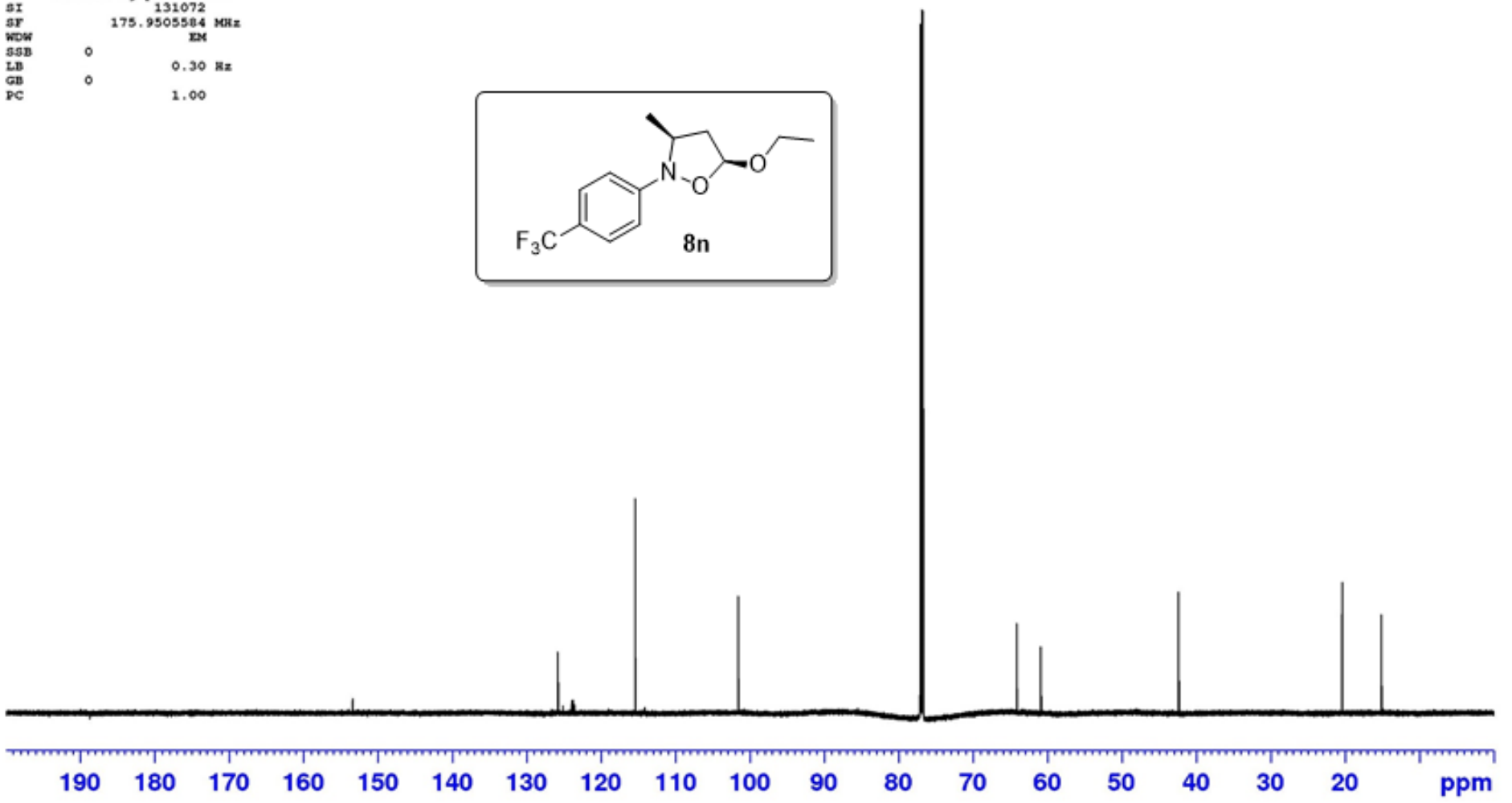
Solvent: $\mathrm{CDCl}_{3}$ SFO1: $700 \mathrm{MHz}$
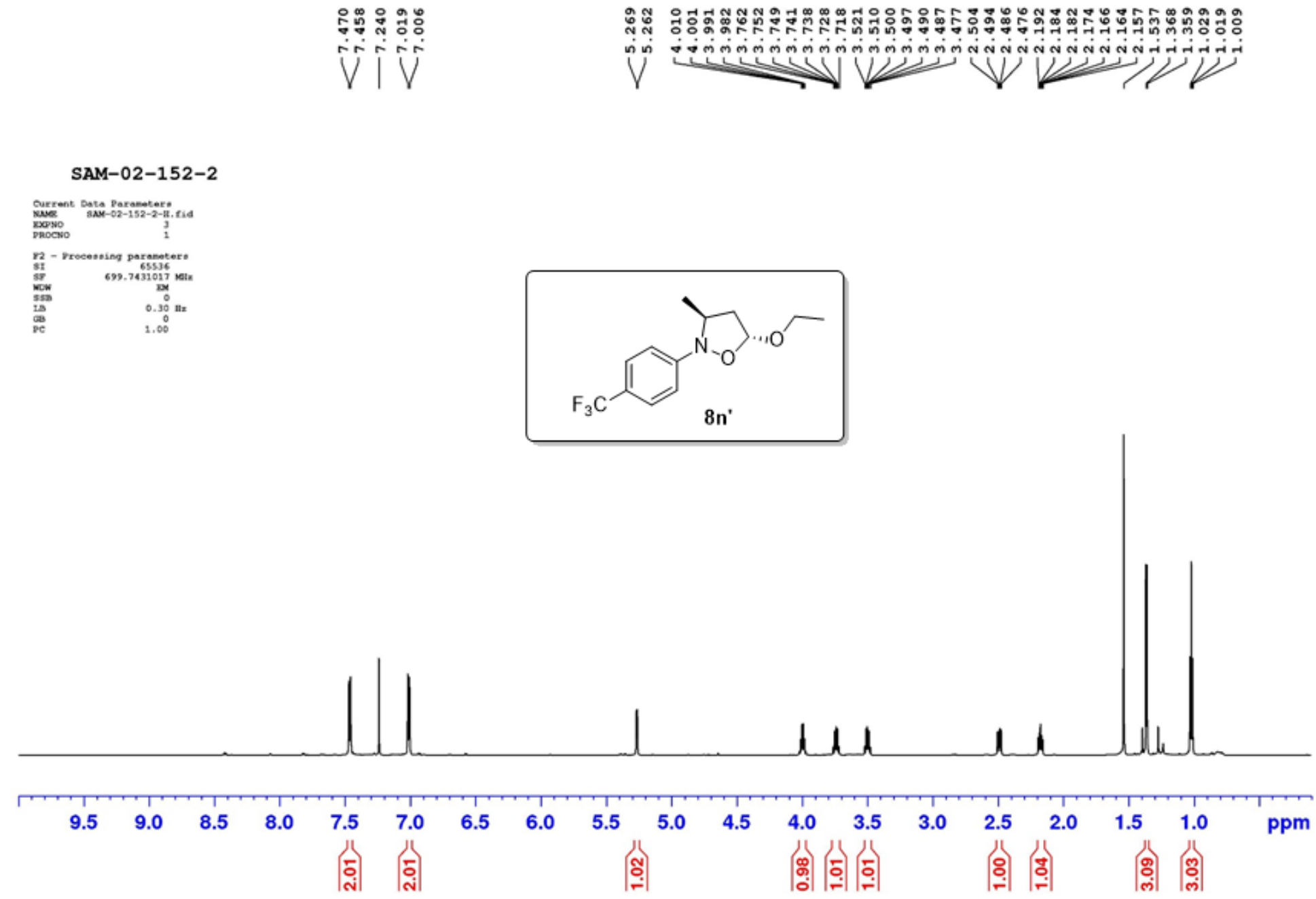
Solvent: $\mathrm{CDCl}_{3}$

SFO1: $175 \mathrm{MHz}$
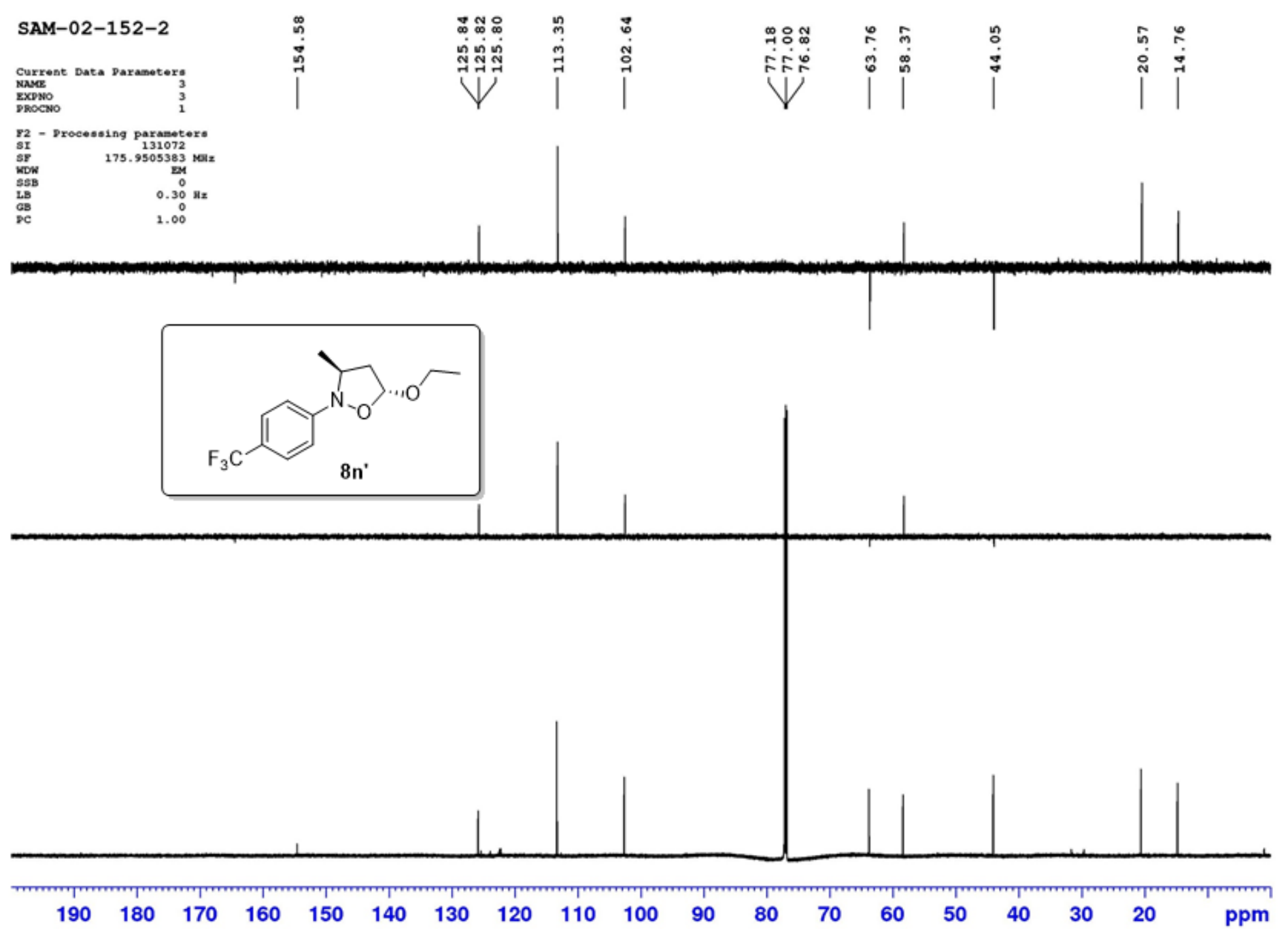
Solvent: $\mathrm{CDCl}_{3}$ SFO1: $700 \mathrm{MHz}$

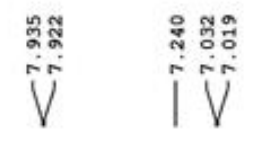

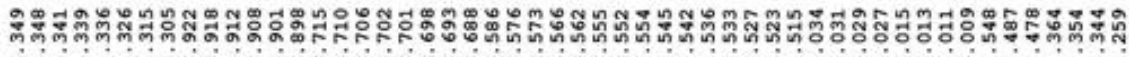

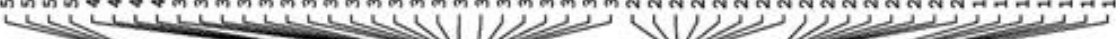

SAM-02-133-1
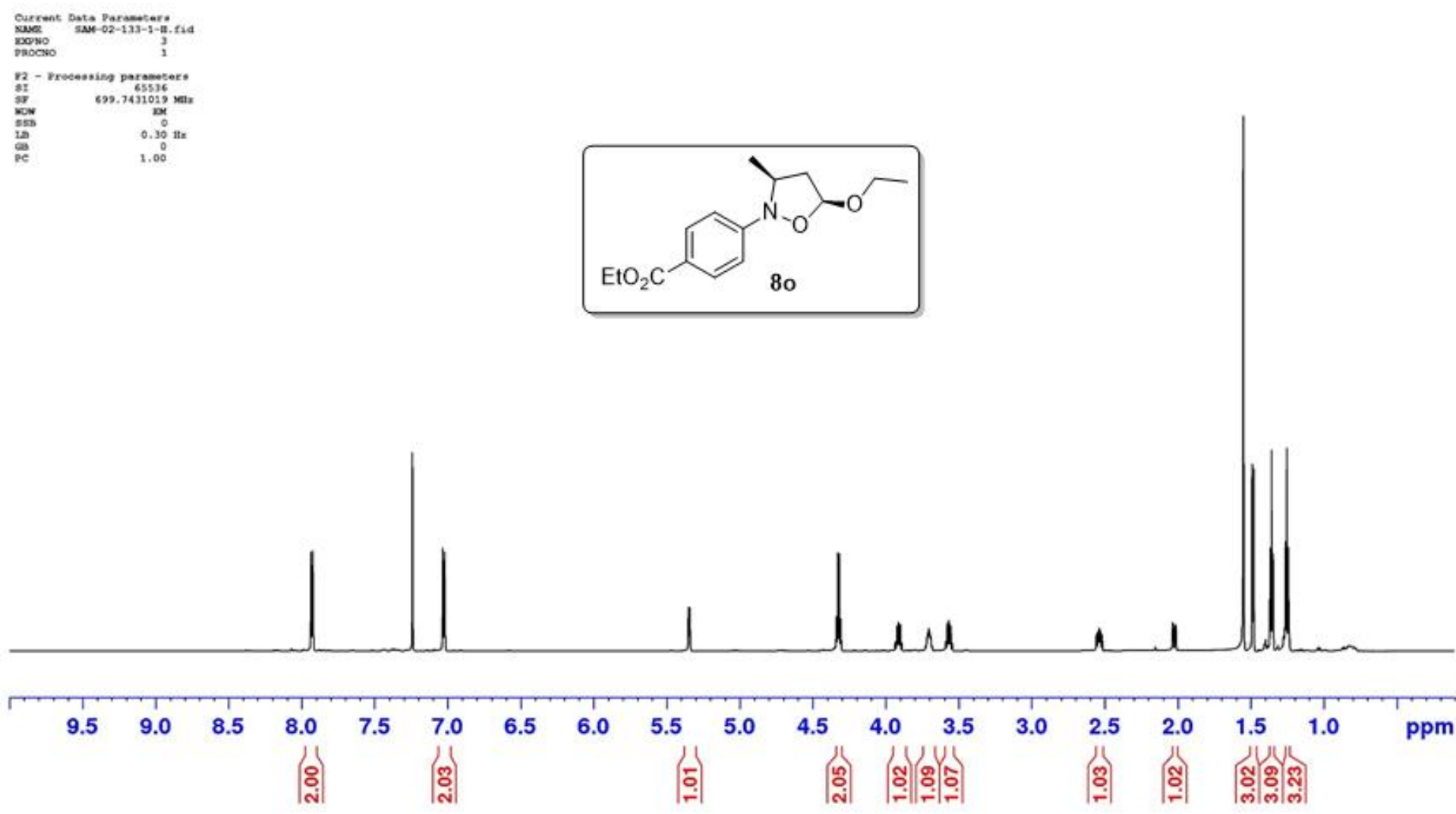
Solvent: $\mathrm{CDCl}_{3}$

SFO1: $175 \mathrm{MHz}$

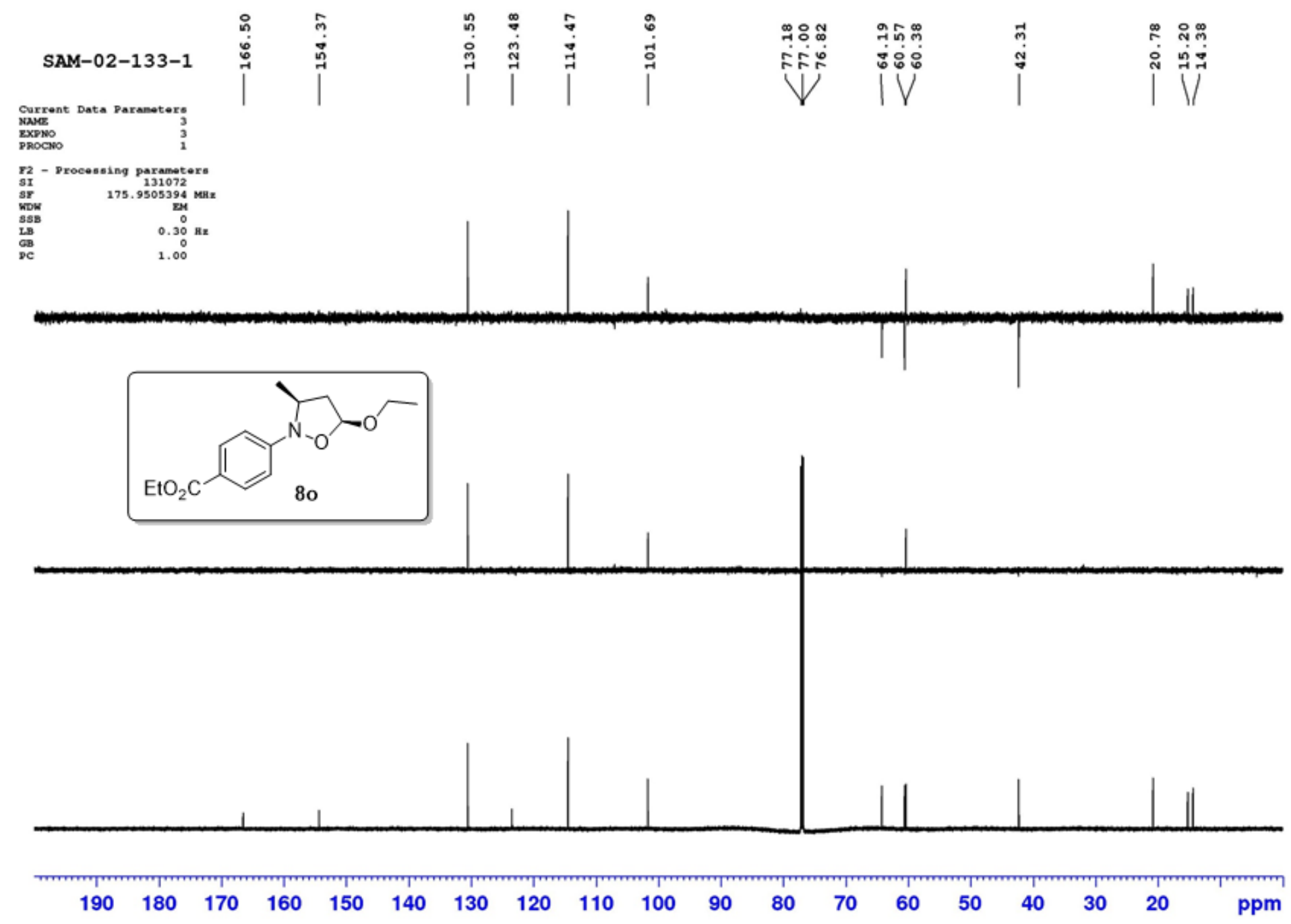


Solvent: $\mathrm{CDCl}_{3}$ SFO1: $700 \mathrm{MHz}$

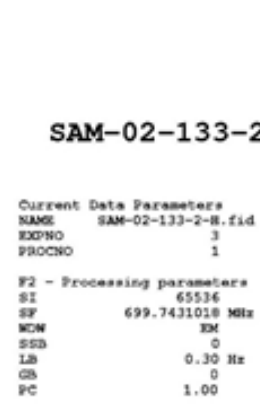
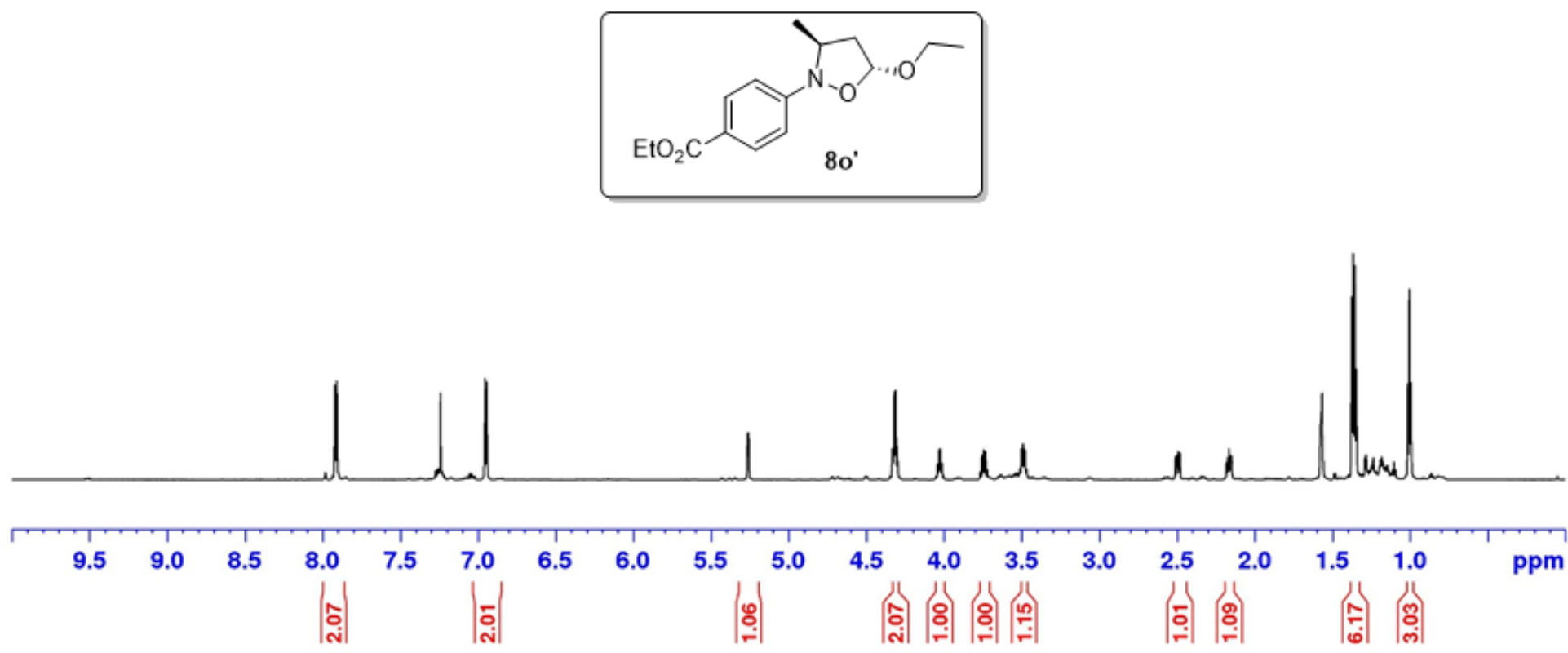
Solvent: $\mathrm{CDCl}_{3}$

SFO1: $175 \mathrm{MHz}$
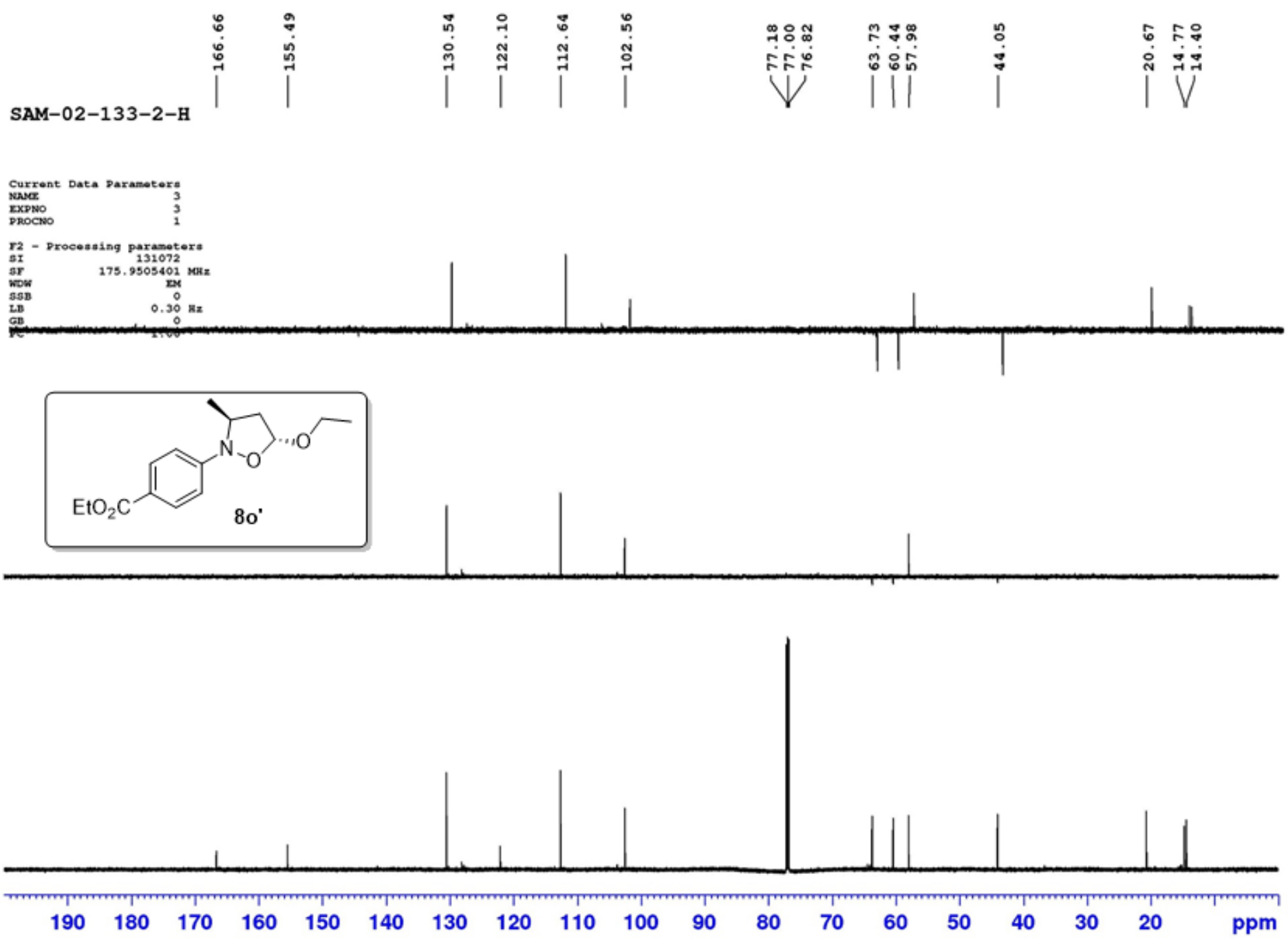